\title{
Solution Based Deposition of Polymide Ablators for NIF Capsules
}

\author{
R. Cook
}

July 11, 2002

U.S. Department of Energy

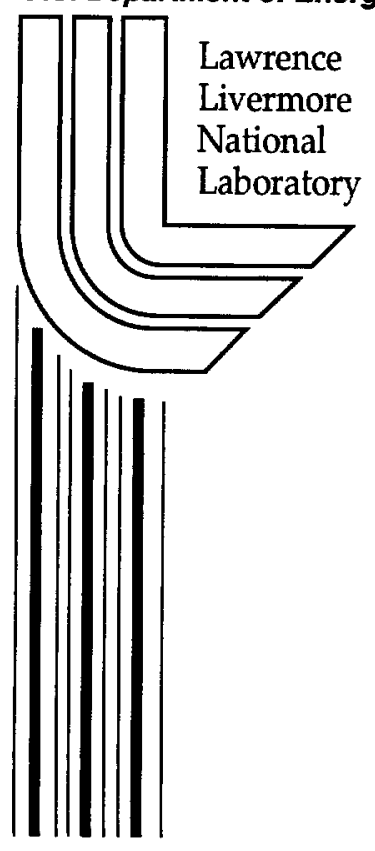

Approved for public release; further dissemination unlimited 


\section{DISCLAIMER}

This document was prepared as an account of work sponsored by an agency of the United States Government. Neither the United States Government nor the University of California nor any of their employees, makes any warranty, express or implied, or assumes any legal liability or responsibility for the accuracy, completeness, or usefulness of any information, apparatus, product, or process disclosed, or represents that its use would not infringe privately owned rights. Reference herein to any specific commercial product, process, or service by trade name, trademark, manufacturer, or otherwise, does not necessarily constitute or imply its endorsement, recommendation, or favoring by the United States Government or the University of California. The views and opinions of authors expressed herein do not necessarily state or reflect those of the United States Government or the University of California, and shall not be used for advertising or product endorsement purposes.

This work was performed under the auspices of the U. S. Department of Energy by the University of California, Lawrence Livermore National Laboratory under Contract No. W-7405-Eng-48.

This report has been reproduced directly from the best available copy.

Available electronically at http://www.doc.gov/bridge

Available for a processing fee to U.S. Department of Energy

And its contractors in paper from

U.S. Department of Energy

Office of Scientific and Technical Information

P.O. Box 62

Oak Ridge, TN 37831-0062

Telephone: (865) 576-8401

Facsimile: (865) 576-5728

E-mail: reports@adonis.osti.gov

Available for the sale to the public from

U.S. Department of Commerce

National Technical Information Service

5285 Port Royal Road

Springfield, VA 22161

Telephone: (800) 553-6847

Facsimile: (703) 605-6900

E-mail: orders@ntis.fedworld.gov

Online ordering: http://www.ntis.gov/ordering.htm

OR

Lawrence Livermore National Laboratory

Technical Information Department's Digital Library

http://www.llnl.gov/tid/Library.html 


\section{Solution Based Deposition of Polyimide Ablators for NIF Capsules}

Work performed under contract to LLNL by

Luxel Corporation

Friday Harbor, Washington

Overview:

Between June 1997 and March 2002 Luxel Corporation was contracted to explore the possibility of preparing NIF scale capsules with polyimide ablators using solution-based techniques. This work offered a potential alternative to a vapor deposition approach talking place at LLNL. ${ }^{1}$ The motivation for pursuing the solution-based approach was primarily two-fold. First, it was expected that much higher strength capsules (relative to vapor deposition) could be prepared since the solution precursors were known to produce high strength films. Second, in applying the ablator as a fluid it was expected that surface tension effects would lead to very smooth surfaces. These potential advantages were offset by expected difficulties, primary among them that the capsules would need to be levitated in some fashion (for example acoustically) during coating and processing, and that application of the coating uniformly to thicknesses of $150 \mu \mathrm{m}$ on levitated capsules would be difficult.

Because of the expected problems with the coupling of levitation and coating, most of the initial effort was to develop coating and processing techniques on stalk-mounted capsules. The program had some success. Using atomizer spray techniques in which application of $\sim 5 \mu \mathrm{m}$ fluid coatings were alternated with heating to remove solvent resulted in up to $70 \mu \mathrm{m}$ thick coatings that were reasonably smooth at short wavelengths, and showed only about a $1 \mu \mathrm{m}$ thickness variation over long wavelengths. More controlled deposition with an inkjet devise was also developed. However difficult technical problems remained, and these problems coupled with the relative success of the vapor deposition approach led to the termination of the solution-based work in 2002.

What follows is a compilation of the progress reports submitted by Luxel for this work which spanned a number of separate contracts. The reports are arranged chronologically, the last report in the collection has a modest summary of what has been accomplished and provides a reasonably starting point for reviewing the work performed.

\footnotetext{
I See S. A. Letts, et al., "Vapor Deposited Polyimide Ablators for NIF: Effects of Deposition Process Parameters and Solvent Vapor Smoothing on Capsule Surface Finish," Fusion Science and Technology 41, 155 (2002) and references therein.
} 
Final Report

\title{
Solution-based methods for the production of polyimide microspheres
}

\author{
June 1997 - September 1998 \\ Luxel Corporation \\ Friday Harbor, WA
}

\section{Introduction}

Luxel Corporation has been under contract to study the use of solution-based polyimide for manufacturing NIF target capsules. Project requirements specify a spherical capsule with a wall thickness of $150 \mu \mathrm{m}$ (enabling the capsule to withstand a fill pressure of 360 atmospheres), with thickness uniformity to be within $1 \mu \mathrm{m}$ and surface smoothness on the order of $300 \AA$. It was the purpose of this study to determine if these requirements can be met using a solution-based technique such as spincoating or spraying. Toward this end, planar films were produced to determine the processing parameters for the production of $150 \mu \mathrm{m}$ thick films. Strength tests were performed to verify that the modified process yielded film strength equivalent to that predicted from tests on submicron films. Next, different methods of application on both planar and 3-D mandrels were explored in order to determine feasibility of utilizing solution-based polyimide for the NIF target capsules (hereafter called microspheres). This effort culminated with polyimide coated brass and $\mathrm{CH}$ spheres supplied to LLNL for evaluation.

\section{Planar Films}

\section{Spincoating planar surface}

Under a 1993 SBIR contract from NASA, Luxel developed and tested various methods for producing submicron polyimide films for use as $\mathrm{x}$-ray filter substrates and pressure windows. This work resulted in the establishment of reliable and repeatable procedures for spincoating polyimide films from polyimide precursors, imidizing films to maximize strength, and releasing films from substrates to produce freestanding foils in the thickness range of $1000 \AA$ to $1 \mu \mathrm{m}$.

This current project represented a major extension of the previous work in the areas of required thickness and spherical shape of the NIF targets. Our work began with spin-coated polyimide films on silicon wafers for evaluation of imidization cure cycles and tensile strength testing. In order to produce thick films using a solution-based process, multicoatings were used to achieve thicknesses greater than $25 \mu \mathrm{m}$. Following each coating, a soft bake was performed before each subsequent coating. Although Hitachi and Dupont (formulators of polyimides and polyimide precursors) recommended this technique, both warned of a skinning effect that traps solvents between layers resulting in a lower film tensile strength. Multiple coatings also have the 
potential of preventing complete crosslinking to take place during imidization. An extended soft bake at a lower temperature and a high-exhaust-flow oven were suggested to alleviate some of these concerns.

Luxel's SBIR work with submicron films identified Hitachi PIQ L100, a polyimide precursor, as potentially appropriate for production of $150 \mu \mathrm{m}$ microspheres. Hitachi PIQ L100 is reported as having a tensile strength of $32 \mathrm{~kg} / \mathrm{mm}^{2}$. Testing under the SBIR contract showed that this formulation does have superior strength. Although several silicon wafers were multicoated using this formulation, it was quickly observed that to achieve $150 \mu \mathrm{m}$ would require nearly 40 coatings. Hitachi had no other marketed formulation with a higher poise of comparable tensile strength. Hitachi PIQ L100 was therefore rejected as a formula for creating spincoated thick films of polyimide.

Dupont's Pyralin LX PI2611D was also tested because of its comparable tensile strength of 35 $\mathrm{kg} / \mathrm{mm}^{2}$ and its much higher viscosity of $110-135$ poise. An imidized film of five multicoats resulted in a thickness of $114 \mu \mathrm{m}$. This film was processed according to the Technical Data Sheet of PI2611D in which the soft bake process consisted of 30 minutes at $100^{\circ} \mathrm{C}$ followed by 30 minutes at $175^{\circ} \mathrm{C}$. The coated silicon wafers were placed on a hot plate for each soft bake. Following the final soft bake, the films were placed in a vacuum oven at ambient temperature. The temperature was increased during a ramp time of two hours to $350^{\circ} \mathrm{C}$. The films were then processed at maximum temperature for one hour. The oven is continuously evacuated and backfilled with argon throughout the imidization process. This venting and filling operation is key to removing solvents and by-products of polymerization. This imidization protocol has been altered considerably from the standard profile customarily used by Luxel for production of thinner films, which is a 20-minute ramp-up from $200^{\circ} \mathrm{C}$ to $325^{\circ} \mathrm{C}$. The slower ramp-up of approximately two hours and a longer duration hard bake of one hour was observed to be necessary to prevent blistering of the films. This cure profile has become the standard for subsequent microsphere testing.

\section{Spraying a planar surface}

Because the spincoating technique could not be directly applied to the fabrication of microspheres, research focused on spray techniques including both ink jet printer technology and a J.E. Meinhard concentric glass nebulizer. Initial results with spraying suggested that a lower viscosity polyimide solution was necessary, therefore Hitachi PIQ L100 was used for all subsequent tests. This precursor was further diluted with N-Methyl-2-Pyrrolidone (NMP) to obtain a desired viscosity.

Initially much hope was placed in utilizing ink jet printer technology. A literature search revealed that this technology is used in making three dimensional prototype machine components with polymeric materials. Many challenges presented themselves, perhaps foremost the difficulty in discovering the requirements of the "ink". Because of the competition among printer manufacturers to hold proprietary their particular technology, it was difficult to ascertain whether any polyimide solution could be compatible with print heads. Three types of ink jet print heads exist: those using heat to cause bubbles to form that are then expelled and guided to a target; 
those using a piezo crystal to vibrate a fluid to cause bubble formation; and those using some form of pump or changing cavity size to expel ink droplets. We briefly experimented with using a standard office machine print head. Unfortunately, the Hewlett Packard (HP) ink cartridge dissolved in NMP, the diluent for Hitachi polyimide precursors. The use of heat to form bubbles is obviously problematic for a thermally cured "ink" such as polyimide. While piezo or pumptype print heads could conceivably work - if fabricated of suitable materials - the expense of obtaining a custom print head for these experiments was prohibitive. A more thorough exploration of other spray methods was undertaken.

The basic apparatus used in spraying a planar surface was a Meinhard nebulizer connected to a syringe filled with a $25 \%$ solution of Hitachi PIQ L100 polyamic acid, and to a filtered and regulated compressed air supply. The nebulizer loaned to Luxel by LLNL gave consistent results due to its self-aligning aerosol and small annular area $\left(0.03 \mathrm{~mm}^{2}\right)$. Five-inch silicon wafers were used as spray substrates. These were mounted to an aluminum disk, which was attached to a stepping motor. This arrangement allowed the substrate to be rotated for while spraying the polyimide solution for some experiments

Initial experiments were performed to determine how smooth the surface of a polyimide film could be made using a solution spray technique. Several spray parameters, including sprayer-tosubstrate distance, amount sprayed (increasing the "wetness" on the substrate), and applied air pressure, were varied to determine the optimal conditions for producing a smooth film.

A distance of approximately three inches between the substrate and the nebulizer was determined to result in good quality films, reducing the "granularity" of the surface while minimizing film sag. Several test films were produced, and it was noted that when the film thickness was sufficiently great, surface tension of the liquid would tend to smooth out the otherwise "pebbly" surface resulting from the application of discrete droplets of solution. Profilometer measurements of the resulting films' surfaces showed an abrupt transition between the rough region and the smooth region. Within the smooth region, roughness was measured to be approximately $20 \mathrm{~nm}$, which is also the measured surface roughness of the underlying wafer.

In addition, a systematic study of nebulizer movement during spraying was performed in an attempt to improve a planar film's uniformity. For these tests, the spray head was fixed perpendicular to a stationary vertical silicon wafer at a distance of three inches. Polyimide solution was sprayed at a pressure of $40 \mathrm{psi}$ for approximately three seconds. The wafer was then dismounted from its support and soft-baked to provide a firm film for profile measurement.

The film thickness and uniformity were measured by scribing a gird on the film, consisting of a line through the center of the film with crosshatching every $1 / 8$-inch. Film thickness measurements were made in the vicinity of each crosshatch using a stylus profilometer. The resulting measurements were plotted to show the thickness distribution. A five-term sigmoidal function was fitted to the data, and showed good correlation: 


$$
t=t_{0}+\frac{a}{\left[1+e^{\left.-\left(\frac{r-r_{0}}{b}\right)\right]^{c}}\right.}
$$

Where $t$ is the film thickness at a point $r$ inches from the center of the spray, $t_{0}=615.2147, r_{0}=$ $0.4638, a=15870, b=-0.0514, c=0.3398$;

Computer simulation was used to test different movement patterns of the nebulizer with respect to a rotating wafer. This exercise demonstrated that the simple spinning motion of the wafer was inadequate to produce a film with better than $\sim 25 \%$ thickness variation regardless of how the nebulizer is moved. A more complex wafer movement scheme, such as a multiple-axis planetary motion, would likely improve this considerably.

\section{Strength testing}

When analyzing different processing techniques, it is important to have a standardized strength test by which to compare the resulting films. For thin film work, Luxel has been successful using a biaxial burst procedure. A freestanding film sample is bonded to a $5 \mathrm{~mm}$ diamter test frame. Gas pressure is applied to one side of the film until it bursts. While this method allows different film preparation methods to be directly compared and offers reliable strength predictions, it does not readily scale to the film thicknesses of interest in this work. Thus, Luxel developed a uniaxial tensile strength test procedure for use with the thicker films of this work. The tensile test device consists of a horizontally oriented stage, similar to an x-axis slide. A pair of film clamps is attached, one jaw being fixed while the other is attached to the moving part. A strain gauge is attached to the fixed jaw and measures the force applied to it.

Several aspects of the design of this mechanism deserve mention. First, the horizontal orientation was used so that the mass of the clamps would not pre-stress the strain gauge. Since it will be useful to compare measurements between thick and thin films, and the thin films will not withstand much stress, a minimum amount of pre-stress is desired. The strain gauge itself (Omega LCL-040) can be selected to accommodate the stress range expected, from 0.25 to 40 lbs. For the thick films being produced for this work, the 40-lb. gauge was used. The lead screw is driven by a stepper motor, and the slide speed is adjustable over a fairly wide range. Finally, the grips are of a half-round design to minimize slippage.

ASTM D 882-95a describes a standardized test method for tensile testing thin plastic sheeting. This standard specifies a specimen size of ten inches, which is impractical for this work due to the difficulty of producing such a large piece of polyimide film. A goal of the present effort is to develop a strength measurement method using a small (one inch long by one-quarter inch wide) gauge length so that measurements can be made on more easily produced sample sizes. Since 
this is much smaller even than the ASTM alternative size of four inches, many tests will need to be made to assess the reliability and limitations of this method.

The first tests were conducted by manually turning the slide screw of the tensile test mechanism while observing the output of the strain gauge on a voltmeter. The results of these tests were encouraging, prompting the addition of the motor drive and automated data collection. The attached figures show the curves generated from several of the subsequent tests. These were made using the ASTM-recommended grip separation speed of $0.00837 \mathrm{in} . / \mathrm{sec}$.

Although many more samples must be tested before conclusions can be made concerning this technique, two observations can be made concerning these results. First, there is apparent repeatability of measurement, as indicated by the nearly identical curves from different samples. If this trend holds, it indicates that this method will indeed satisfy the requirement of providing a point of comparison for different film preparation techniques. Second, the ultimate stress, at the breakage point, corresponds to expected values. The published tensile strength of Hitachi L100, given in (Sanchez \& Letts, in Fusion Technology, July 1997), is $3100 \mathrm{~atm}$. The ultimate stress achieved by both sets of samples in the figures is $3000-3500 \mathrm{~atm}$. Of course, further tests should be performed to prove the viability of this testing method.

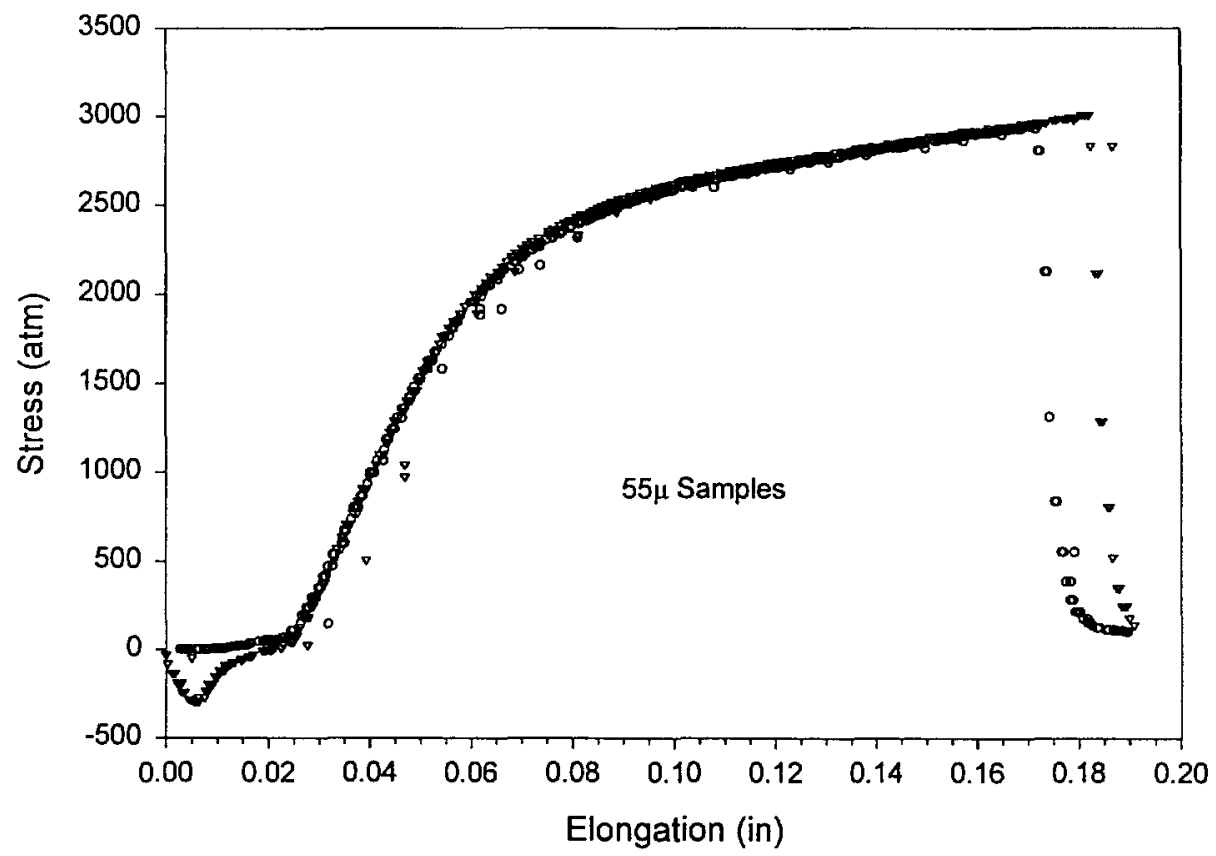




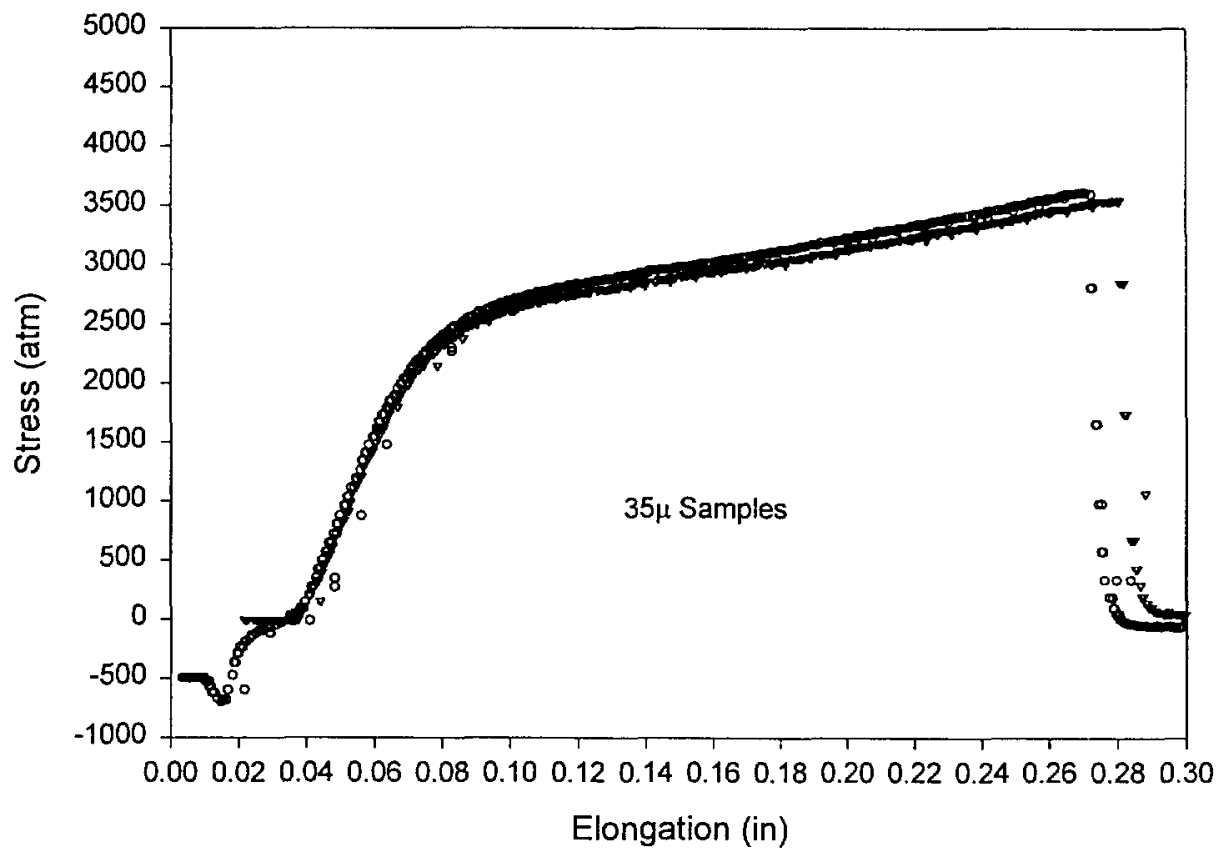

Experience gained from working with planar films allowed us to develop an imidization profile for thick films and provided samples for tensile strength. Further more, it would appear that preparation of thick films by multicoating does not compromise strength. With guidance from LLNL we were encouraged to continue with the spray coatings on non-planar surfaces.

\section{Non Planar Films 0r 3-D Coatings}

\section{Dip coating}

In the last months, Luxel's work has shifted focus away from planar films to three-dimensional films. The films were initially cast on mandrels composed of brass bearings (.093-inch diameter) supported on a 1.25-inch long stem of 31-gauge tubing. The size is approximately that of the microspheres specified for NIF. The stem was inserted into a drilled hole on the bearing, and the same polyimide solution used to coat the mandrels was used to seal the attachment point. This way, the coating could be imidized on the mandrel without detrimental effects due to decomposition of other bonding adhesives at high temperatures.

New tooling was developed that randomizes the surface of the bearing for even heating and fluid flow during the soft bake. The mandrel stem is mounted into a centering chuck that is attached to a drive gear that rides on a $30^{\circ}$-to-vertical bevel gear at a ratio of $8: 1$. This serves to rotate the mandrel in randomized three dimensional pattern. 
New tooling was developed that randomizes the surface of the bearing for even heating and fluid flow during the soft bake. The mandrel stem is mounted into a centering chuck that is attached to a drive gear that rides on a $30^{\circ}$-to-vertical bevel gear at a ratio of $8: 1$. This serves to rotate the mandrel in randomized three dimensional pattern.

The brass mandrels were coated with different dilutions of Hitachi PIQ L100 by using a dipcasting method. Following each individual dip, a soft bake of radiant heat from a controlled filament at $65^{\circ} \mathrm{C}$ for five minutes was performed while the mandrel was rotating on the randomizing device. After a sufficient build up of polyimide, the coated mandrel was placed in the imidization oven. The imidization profile developed in the planar film stage was employed. After imidization, the spheres were inspected and measured for thickness. Typically, planar film thicknesses are measured using a profilometer, however this method is not effective for threedimensional shapes. An ocular micrometer was used to measure the gross film thickness by observing the change in overall diameter of the bearing after coating.

A major difficulty found with dip casting was thickness uniformity. In order to fully coat the mandrel, it had to be dipped to a depth just touching the stem attachment point. The solution viscosity or surface tension was observed to have created a meniscus of excess fluid around the stem. During soft bake, this excess solution flowed back onto the sphere and collected and congealed in the area between the stem and the "equator" of the sphere resulting in thick and thin coating regions. Three samples were provided to LLNL from these early dip casting experiments. LLNL's results as expected, revealed that uniformity of the thickness around the spheres was uneven. The quality of the surface however, was encouraging, as the largest deviation of the samples was $0.6 \mathrm{~nm}$, well within the specifications of the contract. The results of the experiment also showed that surface irregularities of the mandrel could be overcome with enough accumulation of polyimide. Sample coatings on the order of 10-15 $\mu \mathrm{m}$ followed the surface of the underlying mandrel having the same contours. Coatings of $25-60 \mu \mathrm{m}$ on the other hand, did not reveal this effect under microscopic examination.

\section{Spraying}

Having achieved films of $60 \mu \mathrm{m}$ thickness that met smoothness specifications using the dipping method, the next step was to attempt to improve coating uniformity. What's more, we wished to experiment with a spray method because we could imagine ultimately coating mandrels without support stems this way. Luxel began its work to spray mandrels using brass bearings. The mandrels were mounted into the above-described rotating apparatus and as they rotated, the mandrels were sprayed with polyimide. The Meinhard nebulizer was attached to a syringe filled with Hitachi PIQ L100 polyimide solution, as well as to a filtered and regulated compressed air supply. Each spray period lasted 70 seconds using $80 \mathrm{psi}$ of compressed air. The spray period needed to be long enough to cover the entire surface, but not too long as to allow the polyimide solution to run or drip. After each coating of polyimide, as it was still rotating on the apparatus, the microsphere was soft baked using an infrared heat lamp at $74^{\circ} \mathrm{C}$. Multicoat techniques were used to accumulate thicker coatings. After the coating was complete, the microsphere was imidized using the same cure cycle as described in the planar phase. 
Soon after testing began, new mandrels were supplied to Luxel by LLNL. They were polystyrene, poly (alpha-methylstyrene), and $\mathrm{CH}$. The poly spheres supplied were easier to work with and much smoother than the brass bearings. These mandrels were hollow spheres, so drilling a hole for the stem was not a viable option. Stem attachment was accomplished by using a precision XYZ stage for holding the stem next to the sphere and employing a vacuum chuck to hold the sphere. Stems were attached to these spheres with polyimide in the same fashion as with the brass mandrels, but without penetrating the mandrel surface. Once the polyimide was applied, it was soft baked. Unfortunately the polystyrene and poly (alpha-methylstyrene) spheres were not compatible with the polyimide solution components or heat. The $\mathrm{CH}$ spheres were compatible and used for spraying experiments.

While we have not yet achieved full thickness coatings using spraying, our observations of the coated $\mathrm{CH}$ spheres lead us to believe this method is most promising. In general, the spheres appear to be more uniformly coated and more perfectly spherical than the dipped brass mandrels. The surface appears to be smooth under our microscopic examination. Two samples are included with this report for LLNL's evaluation.

Sample \#01C

This sphere was treated with five spray periods of $90 \%$ polyimide solution using 80 psi of compressed air. Each of the spray periods lasted for 70 seconds with soft bakes of five minutes between each period. The sphere was rotated in the randomizing device during spray periods and soft bake. CH mandrel.

Sample \#02C

This sphere was dip-cast, receiving 2 coatings of $90 \%$ polyimide solution with a five-minute softbake after each dip. The sphere was rotated in the randomizing device only during soft bake. $\mathrm{CH}$ mandrel.

The samples provided are samples of method only. While the use of the $\mathrm{CH}$ mandrel improved the quality of the dip-cast coating, there may still be a thickness uniformity issue with coatings employing this method. The spray technique shows considerable promise. We would like help from LLNL to determine the thickness of the coatings achieved with both methods. We estimate sample $\# 01 \mathrm{C}$ to be perhaps 10 or 12 um while $\# 02 \mathrm{C}$ may be on the order of $20 \mu \mathrm{m}$. Evaluation of other features such as smoothness and uniformity would also be helpful.

In conclusion, we have demonstrated that solution-based polyimide films of sufficient strength for NIF target capsules can be fabricated using multicoat techniques. Further, coatings dip cast onto spherical mandrels posses surface smoothness far better than specified. A spray method was developed that may solve thickness uniformity issues observed with dipcasting while also achieving smoothness specification. These achievements certainly suggest that a solution based process for the fabrication of polyimide microspheres may be viable. 


\section{LLNL Purchase Order No. B501348 \\ Demonstration of Coating Levitating Spherical Mandrels}

Final Report

I April 99 through 30 Sept 99

\section{Overview-}

The purpose of this contract is to demonstrate that it is feasible to coat a $2 \mathrm{~mm}$ polymer mandrel with $100 \mu \mathrm{m}$ to $150 \mu \mathrm{m}$ of high strength polyimide, uniformly thick to $\pm 10 \%$. With further development, the result could be uniform strong polyimide target capsules for the National Ignition Facility (NIF).

Experiments conducted during the first two months of this contract showed that acoustic levitation is almost surely required for this application and that a gas-fed spray device (a nebulizer) tends to overpower the levitation forces. It was concluded that a single-axis dual-transducer levitation system would probably provide sufficient levitation stability and that gas-free spray techniques should be pursued. These results were reported in the first progress report included here as Appendix $A$.

Experiments during the second two-month period focused on the application of polyimide using a gas-free spray device. The newest version of the Sonotek ultrasonic atomizer operating at $60 \mathrm{KHz}$ with supplemental air focusing shows great promise. In addition, preparations were made for a week of levitation experiments at CRI (scheduled for August 9-13). These results were reported in the second progress report included here as Appendix B.

Experiments during the third and last two-month period were directed at bringing the best levitation and spray application techniques together in attempts to produce microspheres with uniform coatings of polyimide. It has been shown that the levitation system and ultrasonic atomizer can be configured to produce a desirable mist of polyamic acid in the vicinity of a levitating mandrel, but there is a second tier of more subtle problems to be solved. We are still quite confident that the goals of this project can be achieved but it's more difficult than we had expected. Further details of the work accomplished during this period are given below.

Brief discussion of tests and results for the period from 1 August 99 through 30 Sept 99.

A series of tests and experiments were conducted at Containerless Research Inc. (CRI) during the week of August 9-13. It was found that single axis acoustic levitation with two synchronized transducers works quite well with the LLNL 
supplied mandrels. The levitation is stable and strong. To some degree at least, the rotation can be controlled with a reflector. However, in future efforts, it may be necessary to use a third active transducer to more completely control the rotation. It is anticipated that by using this technique, an even more precise control of polyimide thickness uniformity could be achieved.

The new ultrasonic atomizer with low-pressure air focusing works quite well. For this application, it is far superior to the nebulizer used in earlier experiments. The amount of air and liquid flow is drastically reduced, and it is possible to spray the mandrel with the polyamic acid solution without knocking it out of levitation. Fifteen mandrels were coated at CRI. However, upon further examination, it was determined that the coatings were too thin to be characterized.

It became obvious to us that much more work needed to be done before we could produce samples good enough to be characterized and evaluated against the NIF goals. To this end, arrangements were made to lease the levitation equipment for six weeks so more extensive investigations could be done at Luxel. This has enabled us to try many more experiments than could have been accomplished at CRI during the time remaining on the contract.

After setting up all the equipment at Luxel, the first major task was to try to optimize the spray conditions for delivering the liquid polyamic acid solution for coating the mandrel. It was known from previous experience that the Sonotek atomizer produces a spray with a distribution of droplet sizes from about one to one hundred microns. It is presumed that it would be best to coat the mandrel with the smallest droplets possible in order to achieve the most uniform coating. It was observed that the acoustic field of the levitator tends to repel or at least deflect the spray, and the influence of the acoustic field is more pronounced on the smaller droplets. In other words, the smaller the drop size, the more likely it is to be deflected by the acoustic field. Therefore, great care was taken to try to maximize the amount of fine spray directed toward the levitating mandrel. Steps taken included modifying the atomizer with various extension tubes so that the spray could be injected close to the levitating mandrel inside the first acoustic node. Also, the parameters of liquid and gas flow rates were varied in order to try to maintain laminar flow in the spray since it is believed that laminar flow would be most efficient in delivering many small droplets to the vicinity of the mandrel.

Our efforts were focused on the interactions between the spray and the acoustic field. Eventually we were successful in delivering a satisfactory mist to the vicinity of the levitating mandrel. And yet, even with hours of spraying, we were not achieving any reasonable accumulation of polyimide. Some other factor is preventing the droplets from collecting on the mandrel. In a probable order of likelihood, the possible reasons include: 
- Static electricity is being generated by the spray or the air flow or the rotation of the mandrel and causing the mandrel to repel the liquid droplets.

- The material of the plasma polymer or so-called " $\mathrm{CH}$ " mandrel or something on its surface (presumably left over from the fabrication process) is preventing the polyamic acid solution from wetting it.

- The energy used to atomize the polyamic acid solution is either "cooking it" or partially imidizing it or changing its molecular structure such that it will not adhere to the mandrel.

- The solvent is evaporating somewhere between the atomizer and the mandrel such that a semi-dry spray is being produced, and it will not adhere to the mandrel.

Immediately, a new series of experiments were devised and run. The results were as follows:

- A mandrel was cemented on a wire stalk and mounted in the exact location it would have occupied if levitated. The wire stalk was firmly grounded to the structure of the machine. The levitation equipment was run to simulate, as nearly as possible, the conditions of a levitating mandrel. Also, a low velocity flow of ionized air was introduced to attempt to minimize any static buildup. When the stalk mounted mandrel was sprayed it quickly built up a polyimide coating.

- Mandrels were dipped in the polyamic acid solution, and were wetted. However, there still may be a problem in this area, because wetting appears to depend on the viscosity or concentration of the polyamic acid solution. Concentrations less than $100 \%$ of the polyamic acid solution did not wet the mandrel well. Also it may be that the mandrels vary in their wetability.

- Because it was known that the atomizer gets warm or even hot when operated with low liquid flow rates, a water-cooling jacket had already been added to reduce the potential of partial imidization in the atomizer. Still, it is not known for sure that some sort of partial curing is not taking place because the atomizer does use acoustic energy to form the spray. However, $100 \%$ NMP solvent (which would not be expected to cure) was sprayed and it did not wet the mandrel. This result strongly suggests that partial curing is not the primary problem.

- Many experiments have resulted in a small amount of spray accumulating in small islands on the mandrel. Microscopic 
examination of these samples suggests that "dry spray" is not the problem because the islands of material do not look like granules of coalesced solid drops. Rather, they look like a small amount of liquid has in fact gathered in discrete areas from the amalgamation of droplets.

The results of one of the more significant experiments are shown in Figure 1. The mandrel was stalk mounted, but in position with the levitator running. The spray came from the right. The spray clearly built up a film that completely covered the surface of the right hemisphere that was directly impinged upon by the spray. In fact, the spray coat on that side became so heavy that it slumped, and that caused the irregularities that are seen in the dark shadows along the right upper edge. The more or less vertical line coming down just to the right of the center of the mandrel is where the film became discontinuous at the point where the surface was no longer in the direct path from the source of the spray. The left side of the mandrel has only a smattering of droplets on it. This is from spray droplets that somehow managed to make it "around the corner" and become attached. The brighter spots are from the small droplets. This is especially apparent at the top of the picture where the small droplets cast individual shadows.

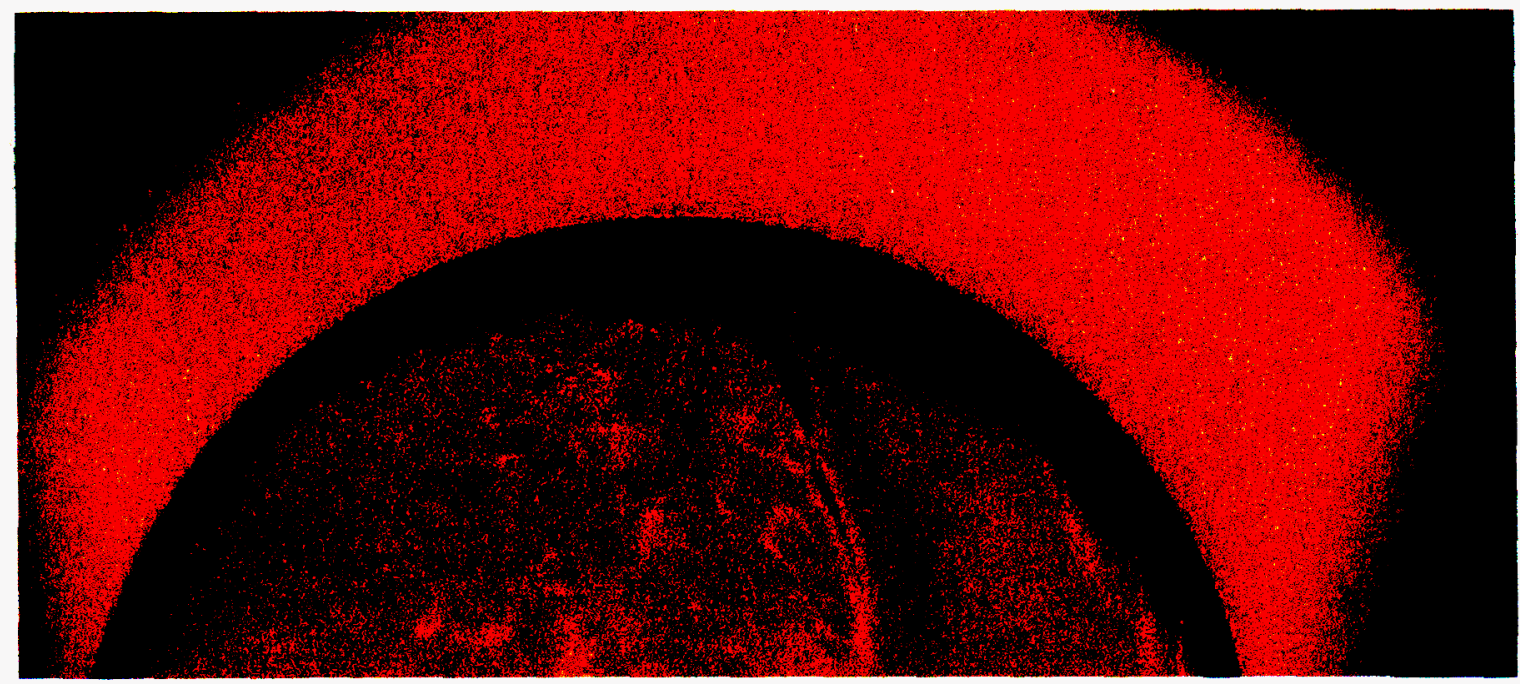

Figure 1. Polyimide coating on a stalk mounted mandrel

We were encouraged by this result, but unfortunately, we have not been able to repeat the results with levitating mandrels. It does appear that the lack of coating is caused by static buildup or the poor wetability of the mandrel or both. Further investigation is required. 


\section{Conclusions-}

At the beginning of this contract, it was generally believed that to be successful, what was needed was a carefully engineered and optimized combination of levitation and spray equipment that would deliver a fine mist of polyamic acid directly to a levitating mandrel. Most of the six months was devoted to this effort, and we have been successful in demonstrating that we can deliver the spray mist through the acoustic field and into the vicinity of the mandrel. This was a major accomplishment. However, we have run into unexpected problems in getting the mist to adhere to the mandrel. We have quickly run a number of experiments, and at this time, we believe the problem is most likely a combination of static buildup and the wetting of the mandrel. In conversations with LLNL staff, we have learned that these issues have come up in the past. Our feeling is that these are solvable problems, and we intend to continue working at no cost to LLNL through the end of October. At that time, we will provide an addendum to this final report, and some sample of mandrels coated with polyimide.

We are exceedingly disappointed that we are not able to deliver coated mandrels at this time. Clearly we were overly optimistic when we took on this challenge. However, we have given it our "best efforts," and we tried to work within the constraints of available time and funding.

We are confident that by the end of October we will be much closer to achieving our original goals. We still believe that the final goal of producing microspheres that meet all the NIF target specifications is achievable using a solution-based approach. The beauty of this approach is that the microspheres will be fabricated without every being touched by a person or a machine. If we did not believe this is possible, we would not be continuing to work using our own funds. By the end of October we will send a proposal for the funding of a continuing project to produce such microspheres. 
Contract financial status-

This contract is out of time and money. While we have not yet achieved the stated objectives, we gave it our "best efforts" and the research results obtained are deemed to be worth the effort expended. This report constitutes the final deliverable and we consider the contract complete and are billing accordingly. The tabulation below shows the contract to be more than $100 \%$ complete financially. Luxel chose to cost share because we believe in the importance of this effort.

UCLLNL Contract No. B501348-modification \#1 Labor effort expended by category and reimbursable costs incurred

Start Date: 04/01/99

Reporting Period: 08/01/99--09/30/99

Financial Report:

$\begin{array}{lrr} & \text { Current } & \text { Cumulative } \\ \text { Engineering } & 5,201.20 & 9,062.60 \\ \text { Corp. Mgt } & 3,663.50 & 7,967.15 \\ \text { Lab Tech } & 167.85 & 415.20 \\ \text { Machinist } & 1,902.80 & 2,130.35 \\ \text { DL Total } & 10,935.35 & 19,575.30 \\ \text { OH @ } & 16,228.06 & 29,049.75 \\ \text { 148.40\% } & & \\ \text { MAT } & 5,554.86 & 8,116.76 \\ \text { SUBS } & 12,924.99 & 25,673.00 \\ \text { ODC } & 21,559.87 & 24,956.11 \\ \text { SUBTOTAL } & 67,203.13 & 107,370.92 \\ \text { G\&A @ } & 12,560.26 & 20,067.62 \\ \text { 18.69\% } & & \\ \text { TOTAL COST } & 79,763.39 & 127,438.54 \\ & & \\ \text { \% Complete Current } & 79.76 \% \\ \text { \% Complete Cumulative } & \end{array}$

- New contract total with Modification 1 


\title{
Appendix A
}

\section{LLNL Purchase Order No. B501348 \\ Demonstration of Coating Levitating Spherical Mandrels}

\section{Progress Report 1}

\author{
1 April 99 through 28 May 99
}

\section{Overview-}

The purpose of this contract is to demonstrate that it is feasible to coat a $2 \mathrm{~mm}$ polymer mandrel with $100 \mu \mathrm{m}$ of high strength polyimide, uniformly thick to \pm $10 \%$, thus creating a strong microsphere which could serve as a target capsule for the National Ignition Facility (NIF). Progress during this two-month period consisted mainly of running a number of experiments involving the spraying of mandrels while being levitated. It had been hoped that aerodynamic, i.e. gas jet, levitation alone might be sufficient, but it appears that because of the extreme light mass of the mandrel, some augmentation by acoustic levitation will be required for stability. But because the simplicity of gas jet levitation is appealing for several reasons, experiments in this area will continue. Also, it appears unlikely that spraying with a nebulizer that uses a gas flow to produce small liquid droplets can be used for this application because the gas flow tends to overcome the levitation forces. Gas-free spraying will most likely be required, and this approach is being investigated and will be pursued in earnest during the next reporting period.

Brief chronology of tests, results and conclusions from significant experiments-

After some initial preparation, a series of experiments were run at Containerless Research Inc. (CRI) during the week beginning April 5th. Both CRI and Luxel personnel participated in these tests. $2 \mathrm{~mm}$ microsphere mandrels were levitated with relative ease in an existing $\mathrm{CRI}$ aerodynamic (gas jet) and 3-axis acoustic levitation system. In all, five mandrels were sprayed using a Meinhard nebulizer. The resultant coatings were sparse and uneven; it appeared that only the largest drops of the spray penetrated the acoustic field to the mandrel. Also levitation was readily disturbed because of the large amount of airflow from the nebulizer. The following conclusions were drawn:

- It appears that aerodynamic levitation alone will not be sufficient in this application and augmentation with acoustic levitation will almost surely be required.

- The gas flow from an air fed nebulizer tends to overpower the levitation forces, and gas-free spraying or coating will probably be required. 
- For this application, it is impossible to maintain cleanliness in any levitation spray system unless it is enclosed in a clean container or a clean room environment. This issue will have to be resolved before clean, sample microspheres can be delivered.

Next, a series of experiments were conducted by CRI pertaining to spray conditions. An artist's airbrush was used to produce a very fine spray. CRI reported that the fine fog-like spray seemed to pass undisturbed through a single axis acoustic field. However, as with the Meinhard nebulizer, the gas flow blew the mandrel out of levitation. Next, a simple experiment was done where cigar smoke was introduced into the acoustic region. CRI reported that the smoke could be moved through the area where the mandrel was being levitated, undisturbed by the acoustic field. Also, using a two-horn vertical axis acoustic system, CRI reported that they were able to use a reflector to cause the rotation of a smooth LLNL-supplied polymer mandrel. The rotation could be induced in different directions by placing the reflector in different positions relative to the mandrel. The following conclusions can be drawn:

- The airbrush and cigar smoke tests seem to confirm that a gas-free spray method should be pursued.

- The rotation of the mandrel is easier to control in a single axis system than it is in a 3-axis system. Also, a single axis system is much less complex than a 3-axis system, so for now it will be assumed that a single axis acoustic system will provide sufficient levitation stability for this application.

Luxel and CRI have both independently conducted a number of experiments pertaining to the feasibility of gas jet levitation for this application and CRI has included the effects of combining gas jet and acoustic levitation. Both companies have tried a number of different configurations. The following conclusions can be drawn:

- $\mathrm{CRI}$ is convinced that because of the low mass of the microsphere mandrel, gas jet levitation alone will not work, and it actually tends to destabilize the acoustic levitation when used in conjunction with it.

- Luxel has not yet been able to achieve stable levitation with a gas jet, but is not ready forego gas jet levitation. One possibility that will be pursued is to inject the polyimide stream directly into the gas jet for delivery to the mandrel.

Luxel has performed some experiments with three nebulizers to determine if this configuration might provide a stable situation. It had been hoped that using three nebulizers mounted 120 degrees apart would tend to equalize the forces on the levitating mandrel, but this did not work because the gas piles up, so to speak, at the center point and then moves outward creating an unstable situation. This approach will not be pursued any further. 
Future plans-

During the next two-month period, equipment options related to gass-free spray devices will be investigated further. There are ultrasonic nebulizers available that will produce a fine fog-like spray without any substantial gas flow. (This is the principal that is used in room humidifiers that produce a very fine low velocity fog.) Also, it is possible that a pure liquid discharge of polyimide could be directed at the levitating mandrel so that the polyimide would accumulate on the mandrel much more quickly than if the mandrel is immersed in a fog of polyimide droplets. Based on what has been learned thus far, the fundamental question of how to coat a levitating mandrel with a uniform layer of polyimide will be readdressed. It should be possible to procure different spraying or coating equipment that is more appropriate to this particular application.

After some progress has been made in choosing a spray method and equipment, another round of joint experiments at CRI will be scheduled to address the issues concerning acoustic levitation and its effect on the coating process. 
UCLLNL Contract No.

B501348

Labor effort expended by category and reimbursable costs incurred

Start Date: 04/01/99

Reporting Period: 04/01/99--05/31/99

Financial

Report:

$\begin{array}{lrr} & \text { Current } & \text { Cumulative } \\ \text { Engineering } & 2,615.40 & 2,615.40 \\ \text { Corp. Mgt } & 2,362.50 & 2,362.50 \\ \text { Lab Tech } & 0.00 & 0.00 \\ \text { Machinist } & 172.05 & 172.05 \\ \text { DL Total } & 5,149.95 & 5,149.95 \\ \text { OH @ } & 7,642.53 & 7,642.53 \\ \text { 148.40\% } & & \\ \text { MAT } & 1,401.00 & 1,401.00 \\ \text { SUBS } & 12,748.01 & 12,748.01 \\ \text { ODC } & 3,308.39 & 3,308.39 \\ \text { SUBTOTAL } & 30,249.88 & 30,249.88 \\ \text { G\&A @ } & 5,653.70 & 5,653.70\end{array}$

18.69\%

TOTAL COST $\quad 35,903.58 \quad 35,903.58$

$\%$ Complete Current

$51.29 \%$

$\%$ Complete Cumulative

$51.29 \%$ 


\title{
Appendix B
}

\section{LLNL Purchase Order No. B501348 \\ Demonstration of Coating Levitating Spherical Mandrels}

\author{
Progress Report 2
}

Overview-

30 May 99 through 31 July 99

The purpose of this contract is to demonstrate that it is feasible to coat a $2 \mathrm{~mm}$ polymer mandrel with $100 \mu \mathrm{m}$ to $150 \mu \mathrm{m}$ of high strength polyimide, uniformly thick to $\pm 10 \%$. The result would be a strong microsphere target capsule for the National Ignition Facility (NIF). Experiments during this two-month period focused on the application of polyimide using a gas-free spray device. In addition, preparations were made for a week of levitation experiments at CRI (scheduled for August 9-13).

Brief discussion of tests and results -

Aerodynamic levitation

While it appeared that the April experiments at CRI ruled out the gas jet as the primary means of levitation, Luxel continued some experiments at our laboratory. The process, if it could be made to work, might be adapted to the high temperature environment required for imidization of the polymer coating. A conical fixture was made to create a more stable gas jet for the levitating sphere. The sphere would levitate within the walls of the cone, not "out in the open" of the space above the gas jet. A filament in an expansion tube allowed for instantaneous warming of the air for soft bake. Brass mandrels could be levitated, but again it was observed that the extra airflow from the spray application of polyamic acid solution using a nebulizer would destabilize the levitation. In the instances when it was possible to apply polyimide, the coating was uneven, causing the sphere to hit the sidewall or fall out of levitation. It was not possible to levitate the $\mathrm{CH}$ mandrels. It seems that they are simply too light, and no combination of airflow or fixture rifling would work. The $\mathrm{CH}$ spheres are also susceptible to static charge buildup, and the airflow of aerodynamic levitation seemed to exacerbate this. A few fixture modifications might address these problems. It was determined, however, that the focus at this time should shift to preparation for the August experiments at CRI.

\section{Gas-free polyimide application}

It was observed at CRI and at Luxel and noted from CRI's subsequently reported results that the gas from the Meinhard nebulizer would blow the mandrel out of levitation in their system. During this reporting period, Luxel researched methods by which we could spray polyimide without the high rate of airflow associated with the nebulizer. Initial experiments used an older Sono-tek atomizer on loan from LLNL. Unfortunately, this device did not uniformly atomize the polyamic acid solution. Large irregular droplets were the result. There was no control over the direction of the spray. The device also created a lot of excess heat that would begin to cure the solution and plug the nozzle. Luxel contacted Sono-tek Corporation, who suggested a newer ultrasonic atomizing nozzle system. Luxel sent samples of polyamic acid solution to Sono-tek and they determined that the solution could be atomized by their nozzle system. Luxel arranged a lease on the new higher frequency system and tests began. 
The new atomizing nozzle system generates a spray mist ultrasonically. The system uses a very small amount of airflow to help in directing the mist. The mist can be focused, depending on the working distance, down to a spot size of about $3 \mathrm{~mm}$. This will permit more efficient spraying of a $2 \mathrm{~mm}$ mandrel with less disturbance to the levitation.

Tests were conducted to get familiar with the operation of the atomizer, to characterize the spray pattern, and to envision how the device might be used in experiments using acoustic levitation at CRI. Various experiments were conducted using the atomizer to create a mist and "collecting" the mist onto glass slides. These tests resulted in determining baseline operation parameters (dilution, working distance, film thickness, and exposure time) for the August experiments. Luxel built a custom housing unit for the atomizer. It was found that a shutter to the spray mist would be more efficient than turning the atomizer on and off. The housing also provides for cooling air to circulate around the nozzle during the softbake sessions, reducing the potential of clogging the nozzle with curing polyamic acid solution.

Once we were confident in the operation of the new equipment, it was decided to try to coat a spherical brass bearing. Two samples were prepared for AFM analysis to be performed at LLNL. The mandrels were supported on 31 gauge wire stalks and were rotated on the randomizing device described in Luxel's final report under contract B339865. The spray was delivered using the Sono-tek atomizing nozzle system. One sample received two separate coats with soft bake sessions between them. The other sample was coated and soft baked continuously for ten minutes. The samples were sent to LLNL for surface roughness evaluation. Unfortunately, it turned out that the coatings were too thin to overcome the brass bearing surface roughness. It is not known at this time whether the new spray application technique yields an equally smooth surface as that made using the nebulizer.

\section{Future plans-}

Joint experiments at CRI are scheduled for August 9-16. These experiments will use a one-axis dual transducer acoustic levitation system. The Sono-tek atomizer will be used to apply the polyamic acid solution to the levitating mandrels. It is hoped that thick (several tens of microns), uniform coatings can be applied to $\mathrm{CH}$ mandrels. The spheres will be soft baked while levitating and brought back to Luxel for imidization. Luxel will analyze the data obtained in the joint experiments after August 16 and will continue experiments and direct $C R I$ to continue studies needed to accomplish the project goals. Gas-free spraying experiments will continue at Luxel to produce samples for surface roughness analysis at LLNL. The project final report and follow-on proposal will be written. 


\title{
LLNL Purchase Order No. B501348 \\ Demonstration of Coating Levitating Spherical Mandrels
}

\section{Addendum to Results of Final Report}

\author{
P.I. Forbes Powell \\ Assistants: Jeff Guckian, Robert J. Wilson
}

1 Aug 1999 through 30 Sept 1999

Plus Oct 
Tests at CRI were completed in early August. Luxel leased the levitation equipment from CRI in order to continue tests at Luxel during September and October. The equipment was set up in a clean bench. Initial tests indicated that the device functioned as it had at CRI. Experiments at Luxel focused on coating a mandrel with polyimide. Two methods were used to coat mandrels: a) polyamic acid mist was delivered into the acoustic well with the levitating mandrel and, b) the mandrel was dipped in polyamic acid solution prior to levitation.

Different dilutions of polyamic acid solution were tested with the atomizer. While the atomizer seemed to be preferable to a nebulizer for spraying polyamic acid solution, tests showed that its use might also be limited. Sono-Tek conducted some preliminary tests and reported success with atomizing polyamic acid solutions up to $25 \%$ in NMP. To further characterize the compatibility of the atomizer with polyamic acid solution, tests were continued at Luxel. A thicker $50 \%$ solution failed to properly atomize. Samples of the mist were collected on glass slides. While the mist visibly deposited onto the slide, when cured, the coating evaporated. This would seem to indicate that the atomizer somehow separated the NMP and the polyamic acid, or polyimide was formed, and the resulting liquid that evaporated was water, or a mixture of NMP and water.

Although the $25 \%$ polyamic acid solution appeared to atomize well, it did show some of the above described separation effects from the atomizer. For the tests at Luxel, a tube was placed over the atomizer annulus to aid in directing the mist. Depositions onto a planar surface that used a tube were compared with those that did not. Droplets were collected onto a glass substrate and soft baked. Polyimide droplets from solution that passed through the 2" long $3 / 8^{\prime \prime}$ diameter tubing measured only $250 \AA$ whereas the droplets formed without the tubing were $475 \AA$ after being soft baked. This suggests that the separation effect is still taking place, but to a lesser degree.

The atomizer annulus uses a low-level air supply to direct the solution once it has been atomized, unlike the nebulizer that used airflow to create the mist. The airflow for the atomizer had to be finely tuned with regulators and an inline needle valve. Airflow of $0.5-2.0 \mathrm{scfh}$ was enough to control the direction of the mist, but not so much that it would knock the sphere out of levitation. With tubing surrounding the annulus, airflow rate was $0.75 \mathrm{scfh}$. Without the tubing, airflow rate was around $1.5 \mathrm{scfh}$ depending on the working distance.

To avoid creating a coulombic charge on the air that was used with the atomizer, a Simco $\otimes$ Top Gun ionizing air gun was utilized on the air supply. The acoustic well was then measured for static charge. The ionized air supply appeared sufficient to neutralize the acoustic well within 1 second of use. Nitrogen and argon gas were also tested, but both gave the same results as air.

One of the major problems with handling the spheres was the amount of static charge that each carried. It was also suspected that the static might 
influence the deposition of the polyimide onto the mandrel. To combat the static a Simco $\otimes$ aerostat XC ionizing air blower was placed in the clean station that housed the levitation device. Using an electrostatic field meter, static charge was measured before and after the use of the ionizing air blower. It was found that the syringe used to dispense the polyimide was the largest offender at over 3.0 $\mathrm{KV} / \mathrm{inch}$ on average. The blower virtually neutralized all charges that were measured before using the ionizing air blower.

The optimal distance between the mandrel and atomizer annulus (without tubing) was $0.5^{\prime \prime}-1.5^{\prime \prime}$. The focal point was at about $0.75^{\prime \prime}$. The mist dispersion would greatly increase after about $1.5^{\prime \prime}$. At $1^{\prime \prime}$ the dispersion was about $0.12^{\prime \prime}$ while the dispersion at 2.0" distance was about 0.2". Earlier tests at Luxel and CRI used 1.5", as there was no tubing around the annulus. Once the levitator was located at Luxel there was tubing placed over the annulus. With tubing, this distance was increased such that the tip of the tube was about $0.5^{\prime \prime}$ outside of the acoustic well. The tubing length ranged from 2 " to 3.5 ". Thus, the working distance from the annulus was in the range $2.5^{\prime \prime}$ to $4.0^{\prime \prime}$. The 2 " tubing length allowed the greatest flow of polyimide. Instead of directing the mist directly into the center of the acoustic well, the mist was directed above and allowed to cascade into the well. Different diameters of tubing were tested, $0.25^{\prime \prime} .0 .375^{\prime \prime}$, and $0.5^{\prime \prime}$. The $0.375^{\prime \prime}$ tubing was the preferred tubing. The larger tubing distorted the acoustic field too much, and the smaller tube didn't allow the polyimide through.

Initial tests showed that polyamic acid solution reacted differently to the bare surface of the $\mathrm{CH}$ sphere than to previously coated regions. If the initial coat was discontinuous, subsequent sprayed coatings preferentially accumulated on the previously deposited polyamic acid and not on the bare mandrel.

Coatings thus took on a mottled and bumpy appearance. By dipping the sphere first, it was possible to coat the entire sphere so that subsequent sprayed layers were more uniform.

To dip a mandrel, it was placed in a polyamic acid solution and submerged. It was then extracted with a .004" diameter tungsten wire. The sphere was then placed into the acoustic well and the wire was withdrawn. Several different concentrations of polyamic acid solution were tested. A $100 \%$ polyamic acid solution proved to be the best for this application. The greater viscosity allowed the polyamic acid solution to spread more evenly than the thinner solutions. Thinner solutions would run to the bottom of the sphere leaving no coating on the upper regions (see sample 107B). 
Soft bake was accomplished using a halogen lamp with aluminum reflectors. A $2.75^{\prime \prime}$ lens was used to focus the light. The intensity of the lamps was controlled with a Variac. The target temperature for soft bake was $60^{\circ} \mathrm{C}$ $80^{\circ} \mathrm{C}$. The soft bake cycle duration was $10-30$ minutes. This process took place while the sphere was levitating.

After the soft bake, more coatings could be applied, or if coating was complete, the sphere could then be imidized. To imidize (or "hard bake") the polyimide, the coated mandrels were removed from levitation and placed into a laboratory oven set at $60^{\circ} \mathrm{C}$ for one hour in preparation for the hard bake. The shells were then placed into a vacuum oven. The $\mathrm{CH}$ shells are not capable of withstanding the standard curing cycle that Luxel uses with PIQ-L100 polyimide so a new cure cycle had to be devised to accommodate this. It was also noted by LLNL that the shells were not able to take temperatures much higher than $300^{\circ} \mathrm{C}$, although to cure the polyimide, the cure cycle temperature must be in excess of $300^{\circ} \mathrm{C}$. To compromise, the cure cycle temperature was established at $325^{\circ} \mathrm{C}$.

It was observed that the vacuum of the WWR oven caused the mandrels to collapse. An alternate cure scheme was developed that involved an inert gas environment rather than vacuum. To fully imidize the polyimide shell onto the mandrel, the samples were placed in the vacuum oven and continuously purged with argon. Once the maximum temperature of $325^{\circ} \mathrm{C}$ was reached, the ramp down to room temperature was prolonged to allow diffusion back into the cooling sphere so that it would not collapse. In order to make sure that the altered cure regime would not be detrimental to the strength of the polyimide, measurements were taken and compared. A spin-cast polyimide film was cured using this method and burst pressure data was obtained and analyzed. The burst pressure was within the parameters of similar films tested at Luxel. This demonstrates that the altered cure cycle is able to fully imidize the polyimide without sacrificing strength. The chart below shows historical data of burst pressures taken from different samples of polyimide imidized using Luxel's standard cure profile. The argon purge burst data show that the cure cycle developed for the microsphere application can produce films equivalent in strength to those produced using the standard cure regime. 
Polyim ide Thickness vs Burst Pressure

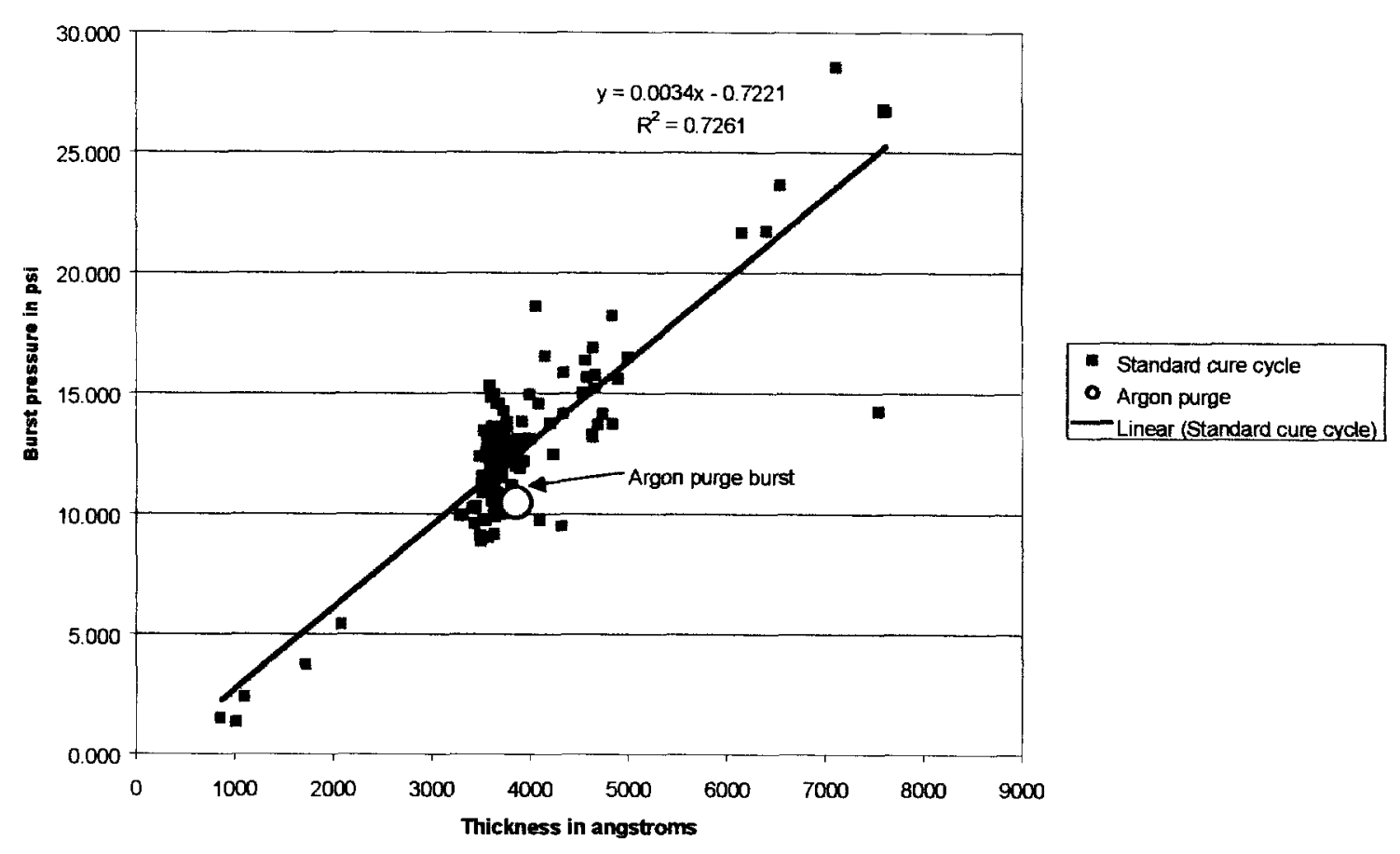

\section{Samples}

All of the following samples are on $\mathrm{CH}$ spheres provided by LLNL. With the exception of samples 105B, 106B, and 107B they were all levitated acoustically in a clean station that was circulated with ionized air. Samples 105B, 106B, and 107B were placed in the acoustic well, but remained attached to tungsten wire support stems. The atomizer power was set at 4.0 watts. The atomizer was water-cooled and tubing was attached to the annulus that directed the polyamic acid mist. The polyamic acid solution was fed into the atomizer at a rate of 0.08 $23 \mathrm{ml} / \mathrm{hr}$. 


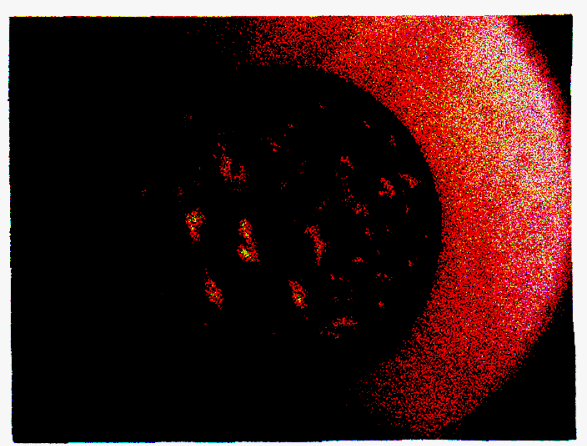

$101 B$

Sample 101B- This sample was sprayed with $25 \%$ solution using the atomizer. It displays about $80 \%$ polyimide coverage. This sample was sprayed for 4.9 hours continuously. The coating was soft baked throughout the deposition. With this sample it is apparent that the polyimide built up in "islands" and did not create a uniform coating.

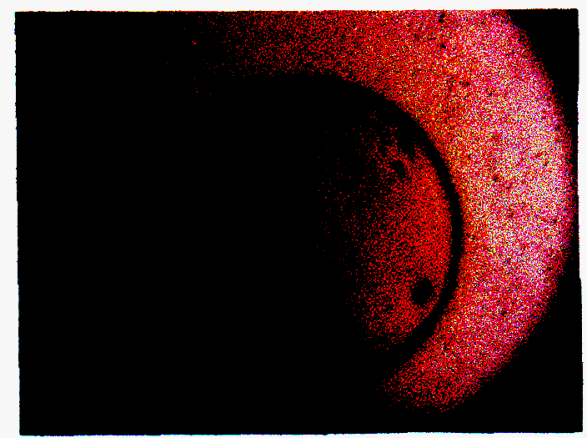

102B

Sample 102B-This sample was coated with seven layers using the dipping method. The sphere appears to have a smooth uniform coating. 


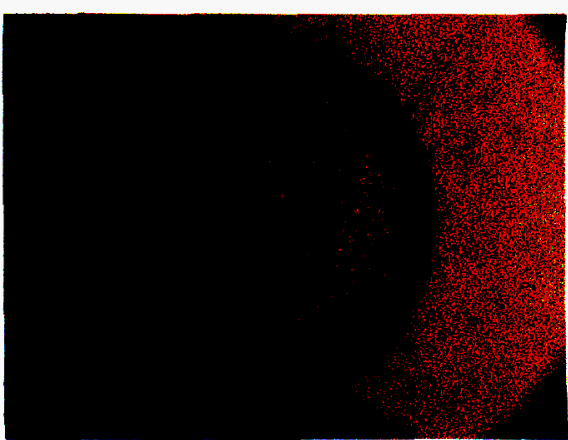

103B

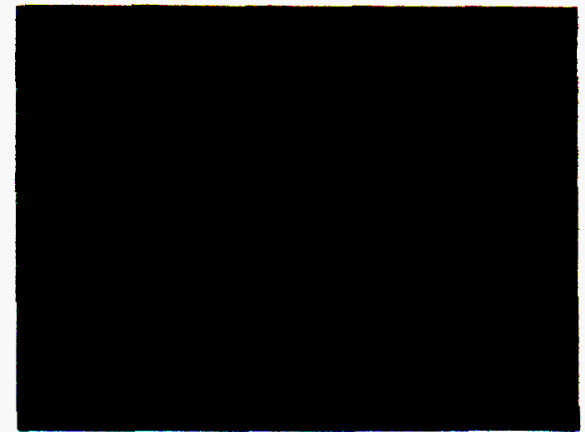

103B

Sample 103B-This sphere is the same as above (sample 102B) with the addition of a sprayed coating. Once the entire surface was covered by dipcoating, it appeared that the polyimide distributed evenly on the sphere.

However, the discrete droplets are seen, causing a rough or bumpy surface. The picture on the right is a close up of the surface. This demonstrates that the polyimide builds up quite differently on the polyimide surface compared to the bare $\mathrm{CH}$ surface (see 101B).

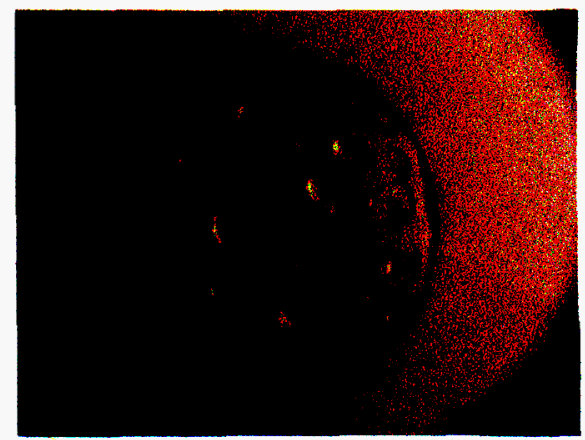

$104 B$

Sample 104B- This sample received one coat by spraying. The droplets did not wet and appearedto bead up away from one another. They also seemed to draw away from the mandrel surface. This sphere was bombarded with ionized air. An ionized air source was directed into the station and the air used in the atomizer was ionized as well. This suggests that static charge is not the problem with the polyimide's inability to wet on the surface of the $\mathrm{CH}$ sphere. 


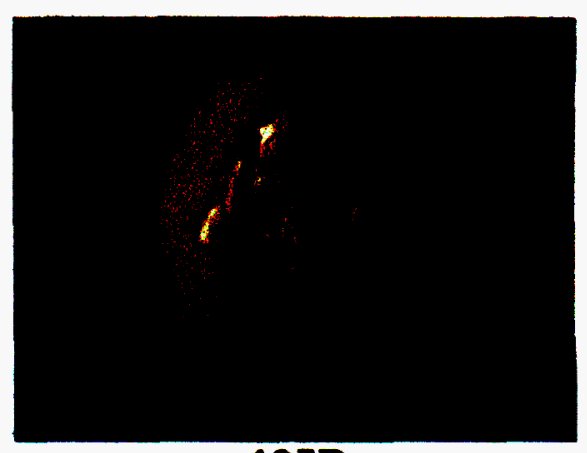

$105 B$

Sample 105B-This sample was sprayed while it was attached to a stem (note the two strands of wire on the lower half of the image). It was placed into the acoustic well with all other variables held constant. This sphere could not rotate. It does appear that this sphere wetted on one side (the lower half in this picture). This sphere was broken during examination, but this photo does illustrate that polyamic acid appears to wet differently on a grounded, non-spinning mandrel. The reasons for this are not known.

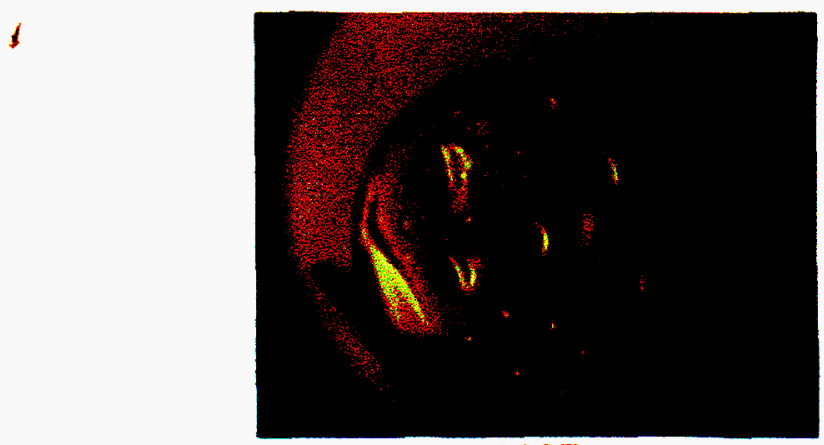

106B

Sample 106B-To test the effects of grounding on the mandrels, a sphere was coated and then grounded to a rod. This sample shows that only the immediate area around the rod is affected. The polyamic acid was attracted to the rod up to about $0.3 \mathrm{~mm}$. The rest of the sphere seemed to be unaffected. When grounded after the deposition, the polyamic acid solution still showed the same wetting problems as observed in sample 104B. 


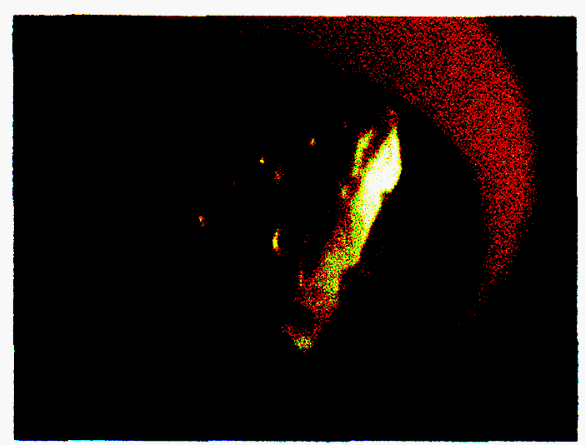

107B

Sample 107B-This sample was $106 \mathrm{~B}$ dipped into $25 \%$ polyamic acid. This demonstrates that the thinner solutions of polyamic acid cannot wet onto the surface of the mandrel. This photo shows the polyamic acid concentrated on the lower right-hand side (the actual bottom), just after being dipped. The thicker $100 \%$ solution does not run down the mandrel as shown with sample 102B.

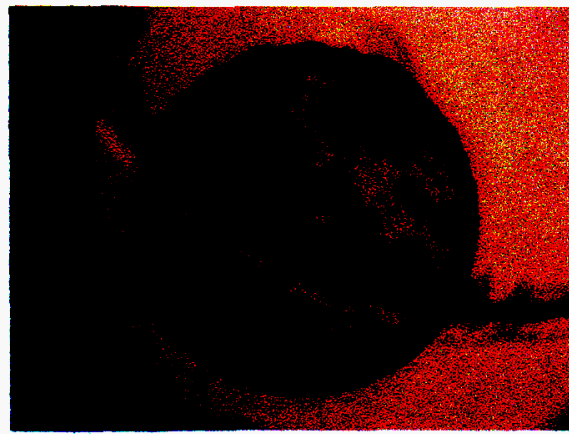

108B

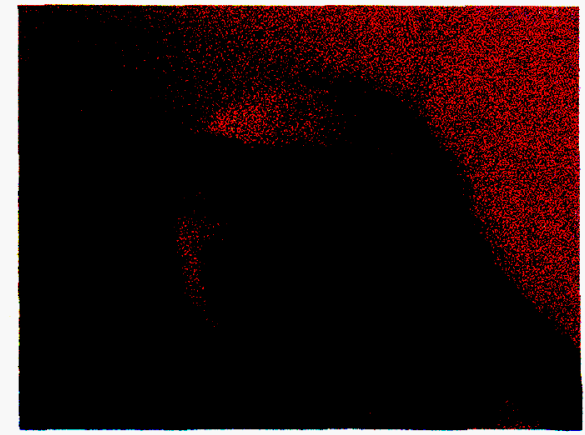

$108 B$

Sample 108B-PIQ-L110 was used on this sample. This polyimide formulation contains adhesion-promoting agents and has comparable strength to PIQ L100. These photos show the same results found with PIQ-L100. These photos were taken before the curing process to show how the polyamic acid builds up in beads. The picture on the right is a close-up that shows the beads in perspective. It is obvious that the PIQ-L110 polyamic acid is not wetting sufficiently to coat the mandrel surface. 


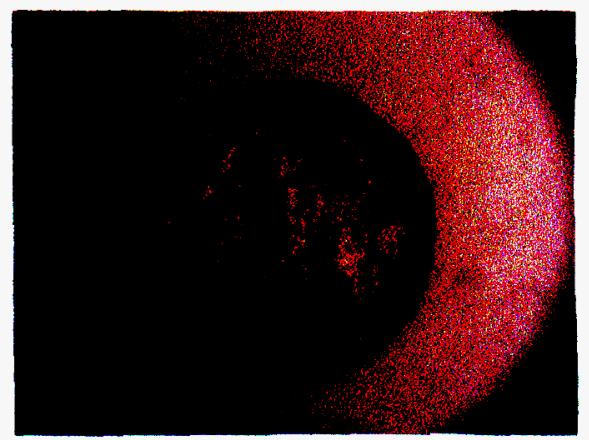

$110 \mathrm{~B}$

Sample 110B-This sample was sprayed for approximately two and a half hours. About $98 \%$ of the mandrel surface was coated by polyimide. The mandrel was observed to spin on a fixed axis, causing the coating to become less uniform with each coat. It appeared that the polyamic acid was more likely to build up on the fast moving areas near the equator.
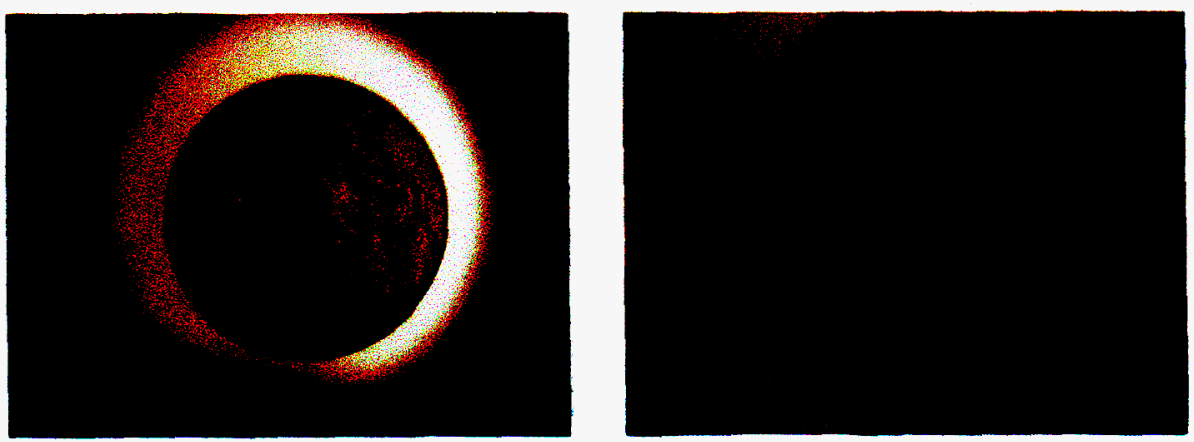

112B

Sample 112B-This sample was dipped once into $100 \%$ polyamic acid and then was sprayed with $25 \%$ polyamic solution for 22 minutes. It was then fully imidized using the modified cure cycle. The hollow $\mathrm{CH}$ mandrel appears to have broken and peeled inward leaving the polyimide coating film to span the break. In this photo, the darker color is the coated $\mathrm{CH}$ mandrel, and the light amber, the polyimide film spanning the break in the mandrel. 


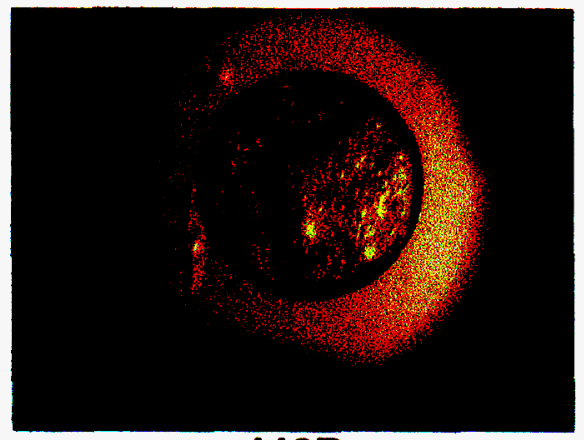

113B

Sample 113B-This sample was dipped once in $100 \%$ polyamic acid. After the first soft baking, it was sprayed for one hour with $25 \%$ solution. This sample is similar to $110 \mathrm{~B}$, but is several microns thinner. The individual polyamic acid droplets are apparent. The lamp was on while the sprayed layers were applied. It is possible that the droplets were soft-baked soon after they contacted the surface of the mandrel.

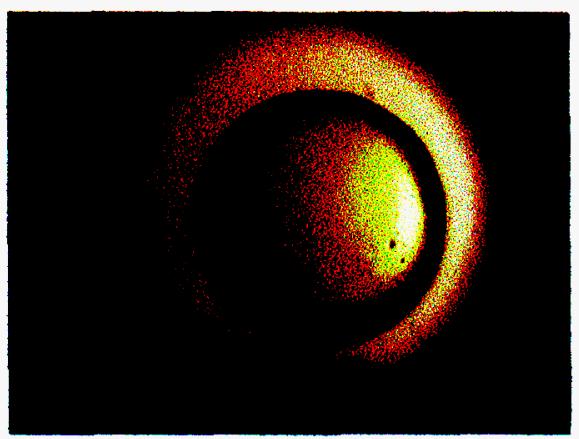

114B

Sample 114B-This sample was dipped 3 times in 100\% solution. Between each dip, the sample was soft baked. The surface is smooth and fully coated. This sample appears to have some symmetrical non-uniformity shown here on the lower region of the photo. It seemed the excess polyamic acid formed a ring around the "equator" in relation to the axis of spin. It was difficult to control the amount of polyamic acid solution in each dipped layer, but it was felt that this sphere used more solution per layer than 102B. If more controlled coat volumes could be realized, then coatings might have less tendency to slump. 


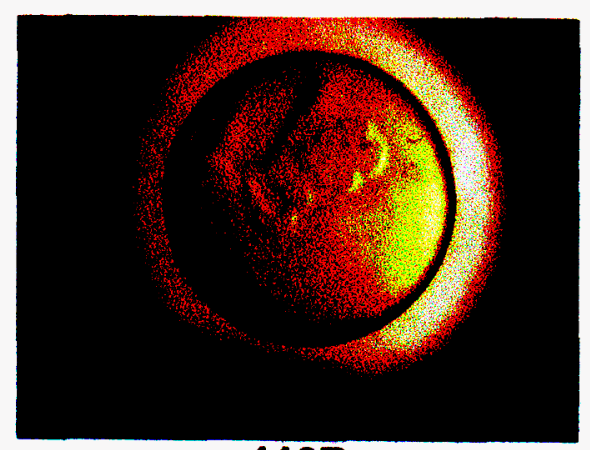

$116 B$

Sample 117B-This sample was dipped once in $100 \%$ solution.

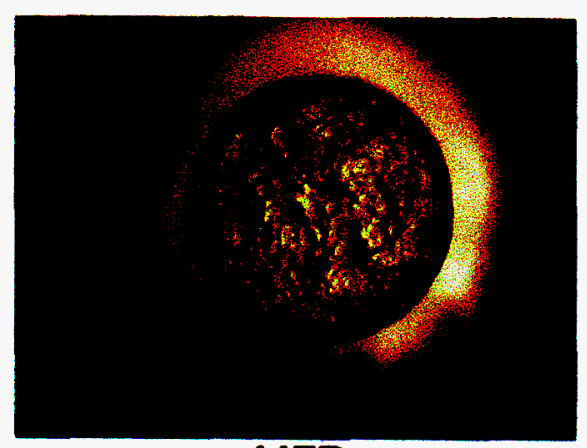

$117 \mathrm{~B}$

Sample 117B-This sample was sprayed with PIQ-L110 for 60 minutes. Sample $108 \mathrm{~B}$ shows the initial reaction of the polyamic acid and the sphere. Sample 117B shows that with continuous deposition the polyamic acid will build up in the same fashion as PIQ-L100.

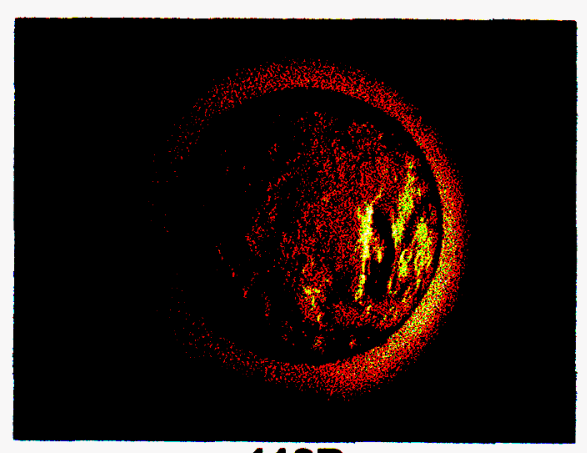

118B

Sample 118B-This sample received two layers using the dipping method with $50 \%$ solution. It was then sprayed with $25 \%$ solution for 25 minutes. 


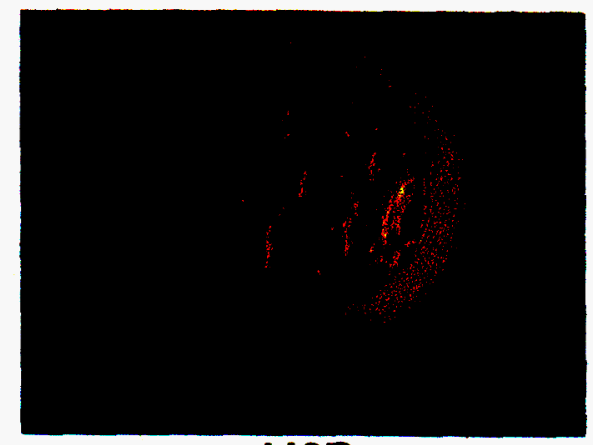

119B

Sample 119B-This sample was sprayed with atomized NMP before being sprayed with $25 \%$ polyamic acid solution. It appeared to wet a bit better, but failed to fully wick around the entire sphere.

In addition to spraying the mandrel surface with NMP prior to coating, other surface treatments were examined as well. Mandrels were subjected to an $\mathrm{O}_{2}$ etch process in the vacuum system. Unfortunately, none survived the treatment intact. In may be that the ion etch imparts too much energy. If this technique is pursued, more experiments to develop a satisfactory process will be required.

It was possible to levitate one sphere with a caved-in area. Because of its shape, the levitation was not particularly stable, and its rotation was not nearly as fast as other spheres. The polyamic acid solution did appear to wet this surface better. Whether this was due to slower rotation or the $\mathrm{O}_{2}$ etch surface treatment could not be discerned.

The samples illustrated are supplied with this report for your examination. The following map will aid in identifying the samples in their holder. 


\section{Progress Report:}

Investigating the Spray Conditions Required to Coat Microspheres with Polyimide

Previous contractual work carried out by Luxel identified a number of specific challenges facing progress toward the goal of manufacturing polyimide NIF target capsules using a solution-based method. Significant progress was made in surrounding an acoustically levitated mandrel with a fine mist of PA (polyamic acid) using ultrasonic atomization, but it was not possible to build up any significant coating on the mandrel. The probable causes of this difficulty were identified in this contract under Task A. Spray coating parameters, as:

1) Ultrasonic atomization. The energy imparted by the ultrasonic atomizer is somehow changing the composition of the PA solution.

2) Dry spray conditions. Since the ultrasonic atomizer creates a fine mist with a mean droplet size on the order of 10 microns or less, it may be that some significant portion of the solvent in the individual droplets is evaporating and that a "dry spray" condition is being created.

3) Wettability. Poor wettability is preventing PA from building up.

4) Static buildup. Static buildup either on the mandrel or the sprayed droplets may cause the spray to be repelled from the mandrel.

Hydrodynamic levitation efforts [contract Task B. Spray while levitating. ] as well as other PA dispensing devices [contract section 4] are also reported on.

Finally, based on the experimental results presented here and on discussions with Bob Cook and Steve Letts during their visit to Luxel on 4 and 5 May 2000, further experiments are proposed to be carried out during the second ( 2 of 3 ) contract period.

1) Ultrasonic atomization. An experimental protocol was established to isolate the effects of atomization on polyimide films and to allow direct comparison with results on standard, unatomized polyimide films obtained by other Luxel researchers. Samples of PA were collected after atomization under various conditions. These conditions were atomization power, PA flow rate, and use of an extension tube. The solutions were then spin coated at 1440 RPM onto five inch diameter silicon wafers using Luxel's standard process. Mass measurements of the wafers were taken before and after spinning, after soft-baking, and again after hard baking in order to determine the relative portion of polyimide precursor in the samples. Determining the relative portion of polyimide precursor in the solution after soft-baking at $75^{\circ} \mathrm{C}$ and again after full imidization at $300^{\circ} \mathrm{C}$ indicates how much NMP solvent was present (lost during soft-bake) and the amount of imidization (water lost during full cure) which occurred in each sample. Film thickness was then measured using Luxel's standard profilometry method. Finally, films were released, mounted on frames and burst tested-again using Luxel's established protocol. 
Table 1 lists the experimental conditions used and the results obtained. Our results indicate that atomizing under the aggravated condition of high power and low PA flow does cause a change in the PA solution that cannot be attributed to the NMP solvent loss typical of soft-baking. Under more normal atomization conditions, we see little change in solution color, viscosity, and film burst pressure results, which leads us to conclude that atomization is an appropriate technique for dispensing PA as long as aggravated conditions are avoided.

Our first indication that something is happening to the chemistry of the PA solution is a change in the color of the samples collected after atomization. High atomizer power and low PA flow rate result in darker solution-dark reddish-brown in the worst case (samples 8 and 10). Our experiments indicate that the dark color is not due to NMP loss (typical of soft-baking) because:

(1) the relative portion of polyimide precursor in the solution does not change significantly, and

(2) the resulting films after spinning were significantly thinner than the control-the solution was less viscous. Fig. 1 shows that our films became thin even though the percent of PA in the solutions did not decrease. We are assuming that soft-baking typically increases the relative percentage of polyimide (i.e. NMP is lost) and the solution becomes more viscous. To test this assumption, an un-atomized sample (\# 11) was soft-baked to a similar dark brown color and run through our experimental protocol. As expected, the relative portion of polyimide precursor in this solution was much higher, the solution was much more viscous and the resulting film was very thick relative to the atomized solutions. Atomization at high power and low PA flow rate produced unusual solutions which were dark colored, without any change in the relative portion of polyimide precursor in the solution, (which occurs during/after soft-baking), and were less viscous, like solutions containing a lower portion of polyimide precursor.

Existing Luxel data on spun film thickness vs. solution percentage is useful for comparing solutions since film thickness is relatively sensitive to small changes in solution percentage (Fig. 2). Samples 8 and 10 were thin, corresponding to solutions that contain approximately $2 \%$ less polyimide precursor, i.e. a $23 \%$ PA solution.

Samples of solution \#10 and the un-atomized control \#12 were soft-baked onto $\mathrm{KBr}$ crystals and sent to LLNL for FTIR analysis. Their results revealed no significant difference between these samples. Either the change in the solution color we see at optical wavelengths does not correspond to chemistry changes revealed at infrared wavelengths (changes in bond stretching and bending), or soft-baking is undoing those changes. Either way, these FTIR results support our conclusion that atomization does not significantly alter PA.

What is happening to these solutions prepared at high atomizer power and low PA flow rate that alters their color and viscosity? Perhaps some imidization is occurring and long polyimide molecules are torn apart by the atomization energy. This could conceivably result in a semicolloidal solution which may explain the thin film / low viscosity result. Soft-baking could then conceivably restore the polymeric solution to a degree such that no changes are observable by FTIR. 
Burst test results indicate that all atomized solutions, regardless of atomization conditions, produced films which burst at slightly lower pressures when compared to Luxel's standard burstpressure data (Fig. 3). However, accounting for experimental uncertainty indicates that these reduced burst pressures are not significant for non-aggravated atomization conditions and marginally significant for aggravated conditions. Not shown in Fig. 3 is sample 11, the unatomized solution that was subjected to soft-bake temperatures until it reached a dark-brown color. This solution was very viscous and produced a thick, $6827 \AA$ film. Surprisingly, this film did not show any degradation in strength, according to our burst test results - polyimide seems to be robust. This result and those shown in Fig. 3 indicate that atomization is an appropriate means for delivering PA.

Factorial analysis reveals the interdependence of atomizer power and PA flow into the atomizer on burst pressures. (Fig. 4) The effect of each factor on burst pressure is shown in figure 5. These results tell us to avoid aggravated atomization conditions, especially high power. We computed the deviation from the standard for each burst data point in order to eliminate dependence on film thickness.

Our experiments indicate that the extension tube has no significant effect on the solution (Fig. 6).

2) Dry spray conditions. Dry-spraying does occur under aggravated conditions. These conditions are high air flow rate and distance. Dry sprayed areas appear like scales under $30 \mathrm{X}$ magnification. Figure 7a shows the cross-over area where "dry-spray" starts to occur. This sample was $5.1 \mathrm{~cm}$ from the atomizer tube-dry-spray occurs under the vapor stream in this instance. A sample $30.5 \mathrm{~cm}$ away was entirely dry-sprayed. A large piece of glass sprayed with the atomizer shows how PA islands get smaller off-axis and far away from the atomizer (Fig. 7b), turning to dry spray when the islands shrink to about $1 \mathrm{~mm}$ or less. It should not be difficult to avoid dry spray conditions, although the pressure gradients of an acoustic levitator may aggravate these conditions. In situ video microscope observations of PA deposition onto mandrels that have been stalk mounted or are levitating hydrodynamically in air shows clearly that dry spray is not a problem.

3) Wettability. The experiment using the Meinhard Nebulizer to spray a brass mandrel mounted on the whirleygig that was performed under the previous contract, was repeated this period using the atomizer - as per the present contract sections 1.3. The atomizer coated a brass mandrel quickly and without difficulty. The PA wet the brass easily with the excess forming a smooth droplet around the bottom (fig. 8). Applying heat (about $80^{\circ} \mathrm{C}$ ) while "whirleygiging" caused polyimide to contract smoothly around the brass sphere. (Fig. 9) The glossy appearing surface of the sphere is similar to what has been reported by previous researchers. The speckled appearance is from the irregularities in the brass sphere.

$\mathrm{CH}$ mandrels, treated on the whirleygig similar to the brass mandrels, behaved very differently. Video microscope observation of PA deposition showed that wetting of PA onto $\mathrm{CH}$ mandrels is 
clearly a problem. Islands of PA were seen to form and compete - actually removing PA from parts of the sphere like water on a waxed surface. (Fig. 10)

Problems with keeping the $\mathrm{CH}$ mandrel glued onto the wire stem and keeping the "whirlygiging" mandrel in focus, led us to abandon the whirleygig in favor of stationary mounting using the vacuum chuck. The whirleygig has been useful for keeping a liquid drop of PA moving around the brass mandrel, perhaps enhancing film uniformity, during soft-baking.

The wettability of different materials, which could be used to pre-treat $\mathrm{CH}$ mandrels, was investigated. Silicon wafers coated with spun, imidized polyimide were treated in different ways and subjected to a drop of 25\% PA. The resulting area of the drop allows us to compare the wettability of these materials. Figures $1-6$ show that NMP which had been spun over the polyimide film and then soft-baked, was the only case which significantly improved wettability. The PA drop wet an area on the NMP sixteen times larger than any of the other cases.

The next step was to attempt to repeat this encouraging result on the $\mathrm{CH}$ mandrel. NMP was sprayed onto a $\mathrm{CH}$ mandrel (fig. 5) and heat was applied from a focused halogen light. The NMP behaved like PA on $\mathrm{CH}$ and unlike PA on brass-it did not wet the $\mathrm{CH}$ mandrel.

Furthermore, when we reached about $50^{\circ} \mathrm{C}$, the $\mathrm{CH}$ mandrel suddenly formed small droplets all over the inside of its surface. (Fig. 6) Raising the temperature higher caused the mandrel to collapse. (Fig. 7) The vacuum chuck may be producing a significant pressure differential across the $\mathrm{CH}$ that promotes diffusion of NMP into the interior of the $\mathrm{CH}$ mandrel. Once inside the mandrel, perhaps hot, evaporated NMP is condensing onto the inner surface, which is cooled by evaporation occurring on the mandrel's outer surface. This explanation seemed to be confirmed when a stalk mounted mandrel showed no condensation when treated similarly. We will avoid using the vacuum chuck in the future or use it at less vacuum with a smaller aperture at the mandrel.

Other attempts were made at drying NMP onto the $\mathrm{CH}$ mandrel. A mandrel soaked in NMP overnight crumpled and was not usable. A mandrel soaked for 1.5 hours, heated to $70^{\circ} \mathrm{C}$, then deposited with atomized PA wet no differently than the untreated mandrel. A mandrel submerged in NMP while the solvent was evaporated away with heat, also wet no better. Finally, heating the mandrel to approximately $100^{\circ} \mathrm{C}$ then spraying atomized NMP onto it caused the solvent to flash evaporate on the surface of the mandrel. A coating of dry NMP was built up this way. PA wet this mandrel much better than the previous efforts.

Dramatic improvement in wettability was obtained on a mandrel supplied by LLNL, which had been O-plasma etched. (fig. 15) An $\mathrm{O}$ etched $\mathrm{CH}$ mandrel was coated, then soft-baked at $60^{\circ} \mathrm{C}$, then re-coated and soft- baked repeatedly for a total of ten layers. This sample will be sent to LLNL for AFM analysis. We hope this sample can then be returned to us where upon we will cure it at $300^{\circ} \mathrm{C}$ and send it back to LLNL for another AFM analysis. This two step procedure will guarantee that we won't loose information if the sample is destroyed during the $300^{\circ} \mathrm{C}$ cure and will also tell us how the surface of the coated mandrel changes from soft bake to full cure. Our immediate task for the second half of the contract period will be to develop a way to oxygen plasma etched $\mathrm{CH}$ mandrels. 
We have obtained samples of an industrial surfactant that is miscible in PA. We will investigate its use as a wetting agent during the $2^{\text {nd }}$ part of the contract period.

4) Static buildup. We never observed any behavior during deposition that could be attributed to electrical charge buildup. Only when inserting a mandrel into levitation was it necessary to neutralize any charge with an ionized air gun to avoid having the mandrel cling to the vacuum chuck or the air tube.

Contract Task B. Spray while levitating. Effort has been spent on hydrodynamic or air levitation of mandrels. A CH sphere was successfully levitated at the end of a tube placed over the air flow coming directly from the atomizer. It was stable, bouncing only slightly (possibly due to inconsistent air supply), and only mildly affected by ambient turbulence. This slight bouncing could be eliminated by "capping" the tube with a glass slide positioned approximately $4 \mathrm{~mm}$ above it. Tube diameter must be $\leq \sim 2.5$ times the diameter of a mandrel or $\sim 5.1 \mathrm{~mm}$ to support stable levitation of a $2 \mathrm{~mm}$ mandrel. A sphere causes enough restriction in the air flow to somehow create a pocket of positive stability, perhaps through a low pressure, Bernoulli venturi effect.

Numerous arrangements for spraying onto a levitating mandrel were explored. A sphere levitated directly on the air coming directly from the atomizer resulted in heavy buildup of PA on the bottom of the mandrel. A filter tube with a $180^{\circ}$ bend was used to filter out "spits" coming directly from the atomizer. In this arrangement, uniform coverage would most likely be obtained by rotating the mandrel. A hole drilled about $2.5 \mathrm{~cm}$ below the top end of the levitation tube, caused PS mandrels to rotate successfully while maintaining levitation stability. $\mathrm{CH}$ mandrels, which lack the surface roughness of PS mandrels, were unaffected. Applying air to the side also had no effect on their rotation.

We also sprayed from the side, separating levitation from spraying for better independent control. Lift was generated by the air passing over the top of the mandrel, causing it to rise up slightly, and contributing, apparently, to stability. It was possible to wet the top of the sphere this way. The most promising arrangement was simply shooting the atomizer straight down onto the levitating mandrel (Fig.16). Large islands of PA accumulated, causing the axis of rotation to start to shift, before an intermittent blast from the atomizer knocked it out of levitation.

Other PA spray devices. [contract section 4] Based on past experience, atomization seems to be adequate for the present. It produces small droplets $(\sim 50-100 \mu \mathrm{m})$ with low velocity $(\sim 1$ $\mathrm{cm} / \mathrm{s}$ ) and does not harm the PA according to the results presented above. However, it is important to consider other options that may become more attractive in the future. Broadly speaking, there are three general types of devices that might be used for spraying PA. There 
are those driven by air pressure, such as the Meinhard nebulizer, those driven by piezo-electric transducers, including both atomizers and ink jets, and those driven by hydraulic pressure such as fuel injectors.

Several suppliers of fuel-injectors for spraying polyamic-acid have been identified: A \& B. One specializes in fluid control engineering, including fuel injectors, and could design and build an injector system for polyamic-acid for us, albeit expensively. The other specializes in diesel injection systems. They tried to discourage us because the tolerances on injectors and pumps are tight and specific to diesel fuel, but they could probably be convinced to sell us something. Bosch would not give out specifications on their fuel injectors, deeming it proprietary information. We are also in contact with companies who specialize in pressure spraying to determine the appropriateness of their techniques.

Microfab Inc. manufactures an ink jet compatible with PA. They claim solutions $\leq 40 \mathrm{cp}$ viscosity are usable. LLNL measured our $17 \%$ solution of PA to have a viscosity of $12 \mathrm{cp}$. We could therefore use a less dilute solution of PA which is desirable for better mandrel coverage.

We were concerned that 100 micron droplets moving at approximately $2 \mathrm{~m} / \mathrm{s}$ out of the inkjet would knock the mandrel out of levitation. The atomizer produces similar sized droplets at two orders of magnitude less velocity that can destabilize hydrodynamically levitated mandrels. However, in this case, the mandrel is being hit by a large number of droplets from the atomizer. In contrast, an ink jet can produce single droplets. It was found, using a conservation of momentum calculation, that a single droplet from an inkjet has 3 orders of magnitude less momentum than is required to knock a mandrel out of acoustic levitation. According to a paper by Paul Nordine from CRI, the maximum velocity that will not cause an acoustically levitated mandrel to be ejected can be estimated as the terminal free fall velocity of the mandrel under gravitational acceleration. We considered the worst case situation, shooting from the side, in a radial direction from the axis of levitation, where, according to Nordine, the radial pressure gradient of the acoustic levitator is $5 \%$ of the axial gradient.

An additional benefit of an inkjet is that $300 \mathrm{DPI}$ of resolution will resolve approximately 20 drops across a $2 \mathrm{~mm}$ mandrel - sufficient to target low PA areas. These specifications will be useful in the future if we can improve $\mathrm{CH}$ wettability, stabilize levitation and a need for targeted drops becomes apparent.

Work to be carried out during the second part of the contract period. Based on our experimental results and discussions with Bob Cook and Steve Letts during their 4-5 May 2000 visit to Luxel, several significant concerns have been identified. Enhancing wettability remains our primary concern. We have two different wetting scenarios to consider: (1) the first PA coat where we need to enhance $\mathrm{CH}$ wettability, and (2) subsequent re-coats where we need to enhance soft-baked polyimide wettability. Our success with the O-plasma-etched $\mathrm{CH}$ mandrels provided by LLNL indicates we need a reliable supply of etched mandrels, either from LLNL, or more favorably, from Luxel, etched in-house using a purchased/loaned plasma etcher or our existing ion gun. Enhancing wettability may also enhance smoothness, particularly in the 10 to 
$100 \mu \mathrm{m}$ range. Furthermore, we want to apply the least amount of solution to minimize flow and slumping (due to gravity) on the surface of the spherical mandrel. How much rotation is required to smooth out the film and avoid slumping? Finally, we need to know what happens during soft-baking, particularly, how much mass is lost.

Our experiments are designed to separate these interrelated issues in order of importance and in a progressive, orderly manner. We are concerned with devising experiments that are quantitative and repeatable, which clearly isolate the issue of interest, and which approximate the levitating mandrel conditions as closely as possible. As per our discussions with Bob Cook and Steve Letts, we plan to rotate small samples mounted vertically whenever possible in order to more closely approximate a rotating, levitating mandrel. We also need to know how to effectively bake in-situ, which early experiments should help us to determine.

Given the above concerns and conditions, our planned experiments are as follows:

1. O-plasma etching

A. We will determine what exposure time to an etching O-plasma is necessary for $\mathrm{CH}$ shells and soft-baked polyimide substrates. In the latter case, optimal exposure time will be estimated by measuring the area covered by mass-calibrated drops of PA onto horizontal, etched substrates. This is not possible in the former case since PA drop volumes are of order of a $\mathrm{CH}$ sphere volume. Qualitatively, the wetting of atomized PA onto an etched $\mathrm{CH}$ sphere is markedly enhanced compared with an un-etched $\mathrm{CH}$ sphere-compare Figs 12 and 15. We are assuming that PA wets etched $\mathrm{CH}$ and polyimide substrates in the same way, such that exposure times determined from the latter apply to the former. This seems reasonable considering that both substrates are polymers.

2. Wetting, mass-loss, and soft-baking

A. We will investigate the effect of two wetting agents, fluorosurfactants, on the wettability of PA onto various polyimide substrates. Wettability will be estimated by (1) measuring the area covered by mass-calibrated drops of PA on horizontally mounted substrates for a given surfactant type and concentration and (2) measuring their contact angles. Fluorosurfactant concentrations are low, typically $\leq 0.5 \%$ by weight.

B. Previous results indicate that $\sim 96 \%$ of the initial PA mass (NMP solvent) is lost during a $\sim 5$ minute soft-bake at $\sim 70^{\circ} \mathrm{C}$ (see Pg. 10, table 1, column 5). This corresponds to Hitachi's claim (makers of PA) that $16 \%$ of our $25 \%$ PA solution contains polyimide precursors, i.e. $4 \%$ by weight. We will measure PA mass-loss as a function of heatingtime and temperature. Given that our current mass-balance detects mass changes $\geq 0.1$ mg, PA on large, 5" Si wafers substrates will be needed/used. PA will be atomized onto these horizontally mounted wafers. These samples will be examined with the profilometer to measure surface roughness (1-D power spectra) as a function of heatingtime and temperature. 
C. Related to A, we will investigate what NMP fraction is necessary for adequate wetting of the next layer. For a given NMP fraction, profilometry will be used to characterize the surface of this next and subsequent layers after soft-baking. When these samples are later imidized, optical microscopy and profilometry we will be used to probe for blistering, which indicates how much NMP needs to be lost. Brown et al.'s (Polymer, 29: 1807, 1987) solution cast polyimide-on-polyimide adhesion studies indicate that interlayer strength is optimized when the $1^{\text {st }}$ layer is cured at $200^{\circ} \mathrm{C}$ prior to $2^{\text {nd }}$ layer depositionthe boiling point of NMP. If increased adhesion corresponds to increased $E_{s}^{P}$ (soft-baked polyimide surface energy), then the condition for wetting,

$$
E_{s}^{P A}+E_{i}^{P A-P}<E_{s}^{P},
$$

indicates that soft-baking at $200^{\circ} \mathrm{C}$ may be optimal for wetting PA onto polyimide ( $E_{s}^{P A}$ is PA surface energy and $E_{i}^{P A-P}$ is PA and Polyimide interfacial energy).

3. Vertically rotating disks spray-coated with PA using a stationary atomizer:

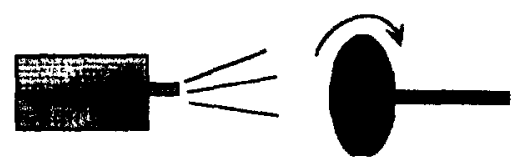

The motivation for this arrangement is to study, in a quantitative manner, conditions-\% PA solution, substrate type, surfactant addition, soft-bake temperature, and heating timenecessary for successful layering of soft-baked polyimide onto a surface that is similar to that encountered when spray-coating PA onto a $\mathrm{CH}$ sphere. An IR heat lamp will be at a fixed distance to uniformly irradiate the surface. The surfaces will be large enough to avoid meniscus edge effects, yet small enough to be uniformly heated by a focused heat lamp: $\sim 10 \mathrm{~mm}$ diameter. The surface will be coated for a measured period of time and then softbaked to determine the rate of deposition (knowing what \% of mass is lost during soft-bake), surface uniformity (slump) and surface roughness (1-D power spectra) using a profilometer. The temperature at the surface will be measured with a thermocouple and/or IR sensor having a known focal length and field of view.

\section{A. Slumping}

Even though a particular \% PA solution, substrate type, and surfactant addition may maximize wettability and minimize the amount of material needed, these conditions may not minimize slumping due to gravity. These experiments are designed to explore this issue.

a. How much of a problem is slumping and how much rotation is needed to overcome it? To answer the first question, our initial experiments will use non-rotating, vertical 
samples. Rotation rate will be increased in subsequent experiments. Small samples will be mounted vertically and deposited on from the side until a fully wet condition occurs, as seen by a video microscope. We will soft-bake using a heat lamp. These soft-baked polyimide and possibly O-etched samples will be sprayed with different \% PA solutions, with and without a surfactant additive. Profilometry should give us thickness gradients and surface roughness, from which we can learn which factors are important in controlling flow due to gravity around the spherical mandrel.

Will our results correlate to a $2 \mathrm{~mm}$ diameter sphere? An extreme situation occurs at the equator of the mandrel where gravity and flow are in the same direction. Vertically mounted samples sprayed on from the side reflect this case. The more manageable case of a rotating disc is analogous to the spherical mandrel rotating around a single axis. Our results will at least give us a relative answer as to which deposition conditions minimize slump. At most, if we assume that flow is a local phenomenon acting over a length scale $\leq 2 \mathrm{~mm}$ (?) and we are careful to make our measurements at the same place we observed during deposition, we can perhaps make an estimation of the amount of non-uniformity which might occur around the mandrel. 
Table 1

\begin{tabular}{|c|c|c|c|c|c|c|c|c|c|}
\hline $\begin{array}{c}\text { Sample } \\
\#\end{array}$ & $\begin{array}{l}\text { Power } \\
(W)\end{array}$ & $\begin{array}{l}\text { Poly flow } \\
\text { (mi/h) }\end{array}$ & Tube & $\begin{array}{l}\text { \% poly in } \\
\text { solution } \\
\text { after } \\
\text { soft-bake }\end{array}$ & $\begin{array}{l}\text { \% poly in } \\
\text { solution } \\
\text { after full } \\
\text { cure }\end{array}$ & $\begin{array}{c}\% \\
\text { difference: } \\
\text { soft to full } \\
\text { cures }\end{array}$ & $\begin{array}{l}\text { Spun film } \\
\text { thickness } \\
\text { (A) }\end{array}$ & $\begin{array}{c}\text { Relative } \\
\text { film } \\
\text { density }\end{array}$ & $\begin{array}{c}\text { Burst } \\
\text { pressure } \\
\text { (PSI) } \\
\text { (std dev.)) }\end{array}$ \\
\hline \multicolumn{10}{|l|}{$\begin{array}{l}3 / 14 / 00 \\
\text { solution }\end{array}$} \\
\hline 1: control & - & - & - & 3.12 & 2.85 & 0.27 & 1036 & 1 & $1.9(0.1)$ \\
\hline 2 & 2 & 1 & - & 4.20 & 2.80 & 1.40 & 1144 & 0.86 & $1.9(0.1)$ \\
\hline 3 & 10 & 1 & - & $9.33 ?$ & $6 ?$ & $2.44 ?$ & 1048 & 1.46 & $1.9(0.1)$ \\
\hline 4 & 20.2 & 1 & - & & 4.91 & & 1188 & 1.54 & $2.0(0.1)$ \\
\hline 5 & 4 & 1 & + & 3.71 & 2.12 & 1.59 & 1098 & 0.72 & $1.0(1 \mathrm{pt})$. \\
\hline 6 & 18.3 & 1 & + & 3.63 & 2.46 & 1.17 & 1129 & 0.83 & $1.6(0.3)$ \\
\hline 7 & 4 & .05 & + & 4.50 & 3.33 & 1.17 & 1435 & 0.89 & $2.2(0.2)$ \\
\hline 8 & 19 & .05 & + & & 3.07 & & 759 & 0.91 & 1.0 (1pt.) \\
\hline \multicolumn{10}{|l|}{$\begin{array}{l}3 / 21 / 00 \\
\text { solution }\end{array}$} \\
\hline 9 & 4 & .2 & - & 4.07 & 2.56 & 1.51 & 1084 & 0.91 & $2.1(0.1)$ \\
\hline 10 & 18 & .05 & - & 4.57 & 2.83 & 1.74 & 741 & 1.02 & $1.0(0.1)$ \\
\hline $\begin{array}{l}11 \\
\text { Unatomized } \\
\text { \&soft- } \\
\text { baked }\end{array}$ & & & & 10.90 & 7.15 & 3.75 & 6827 & 1.04 & $22.7(0.6)$ \\
\hline 12 control & - & - & - & 4.47 & 2.77 & 1.70 & 1045 & 1 & $1.7(0.1)$ \\
\hline
\end{tabular}

All samples at 2 SCFH air flow and $25 \%$ polyamic-acid solution.

Figure 1

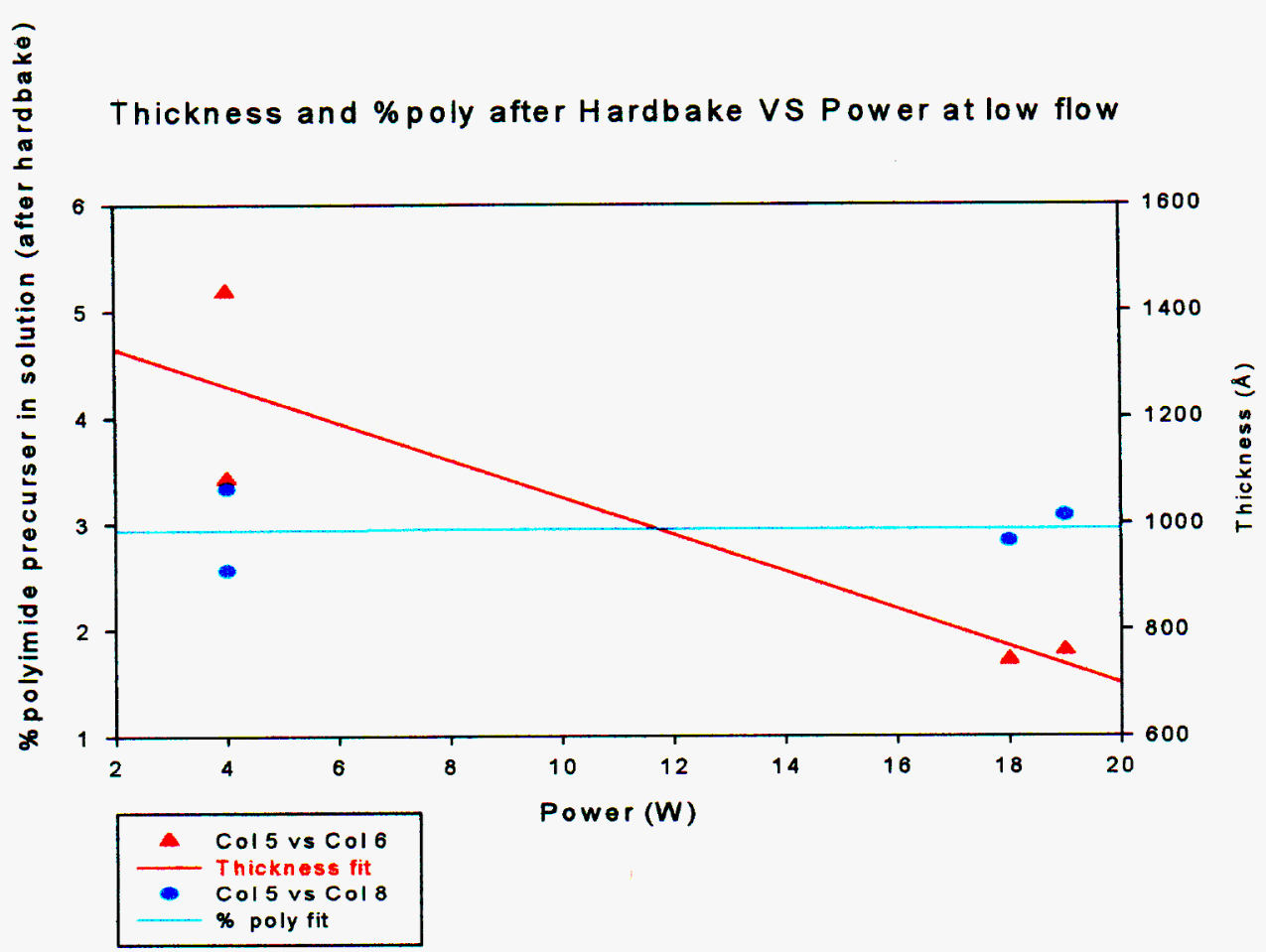




\section{Figure 2}

\section{thickness Vs. \% polyamic solution @ 1400 RPM linear approximation}

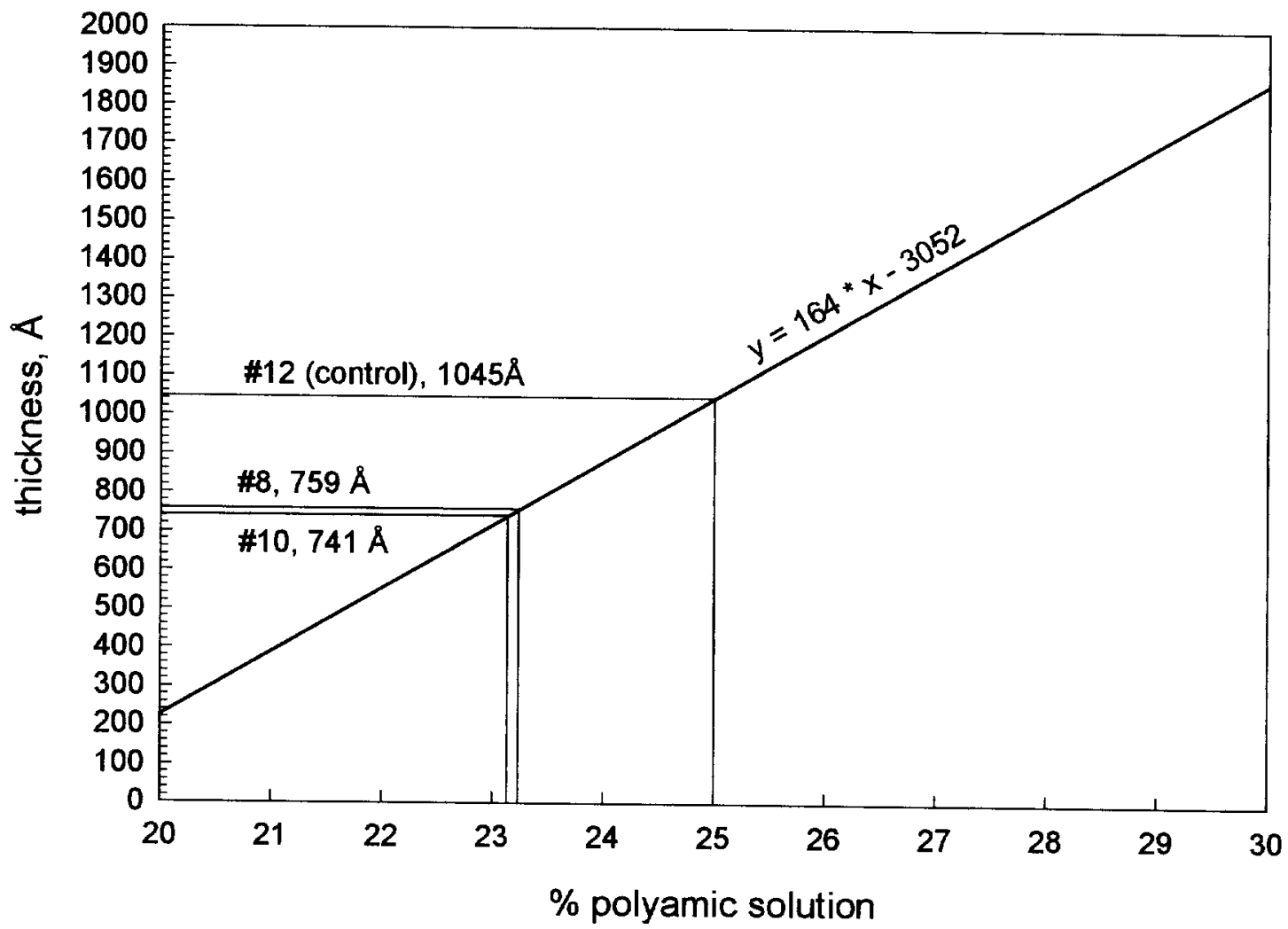




\section{Figure 3}

\section{burst-pressure Vs. thickness}

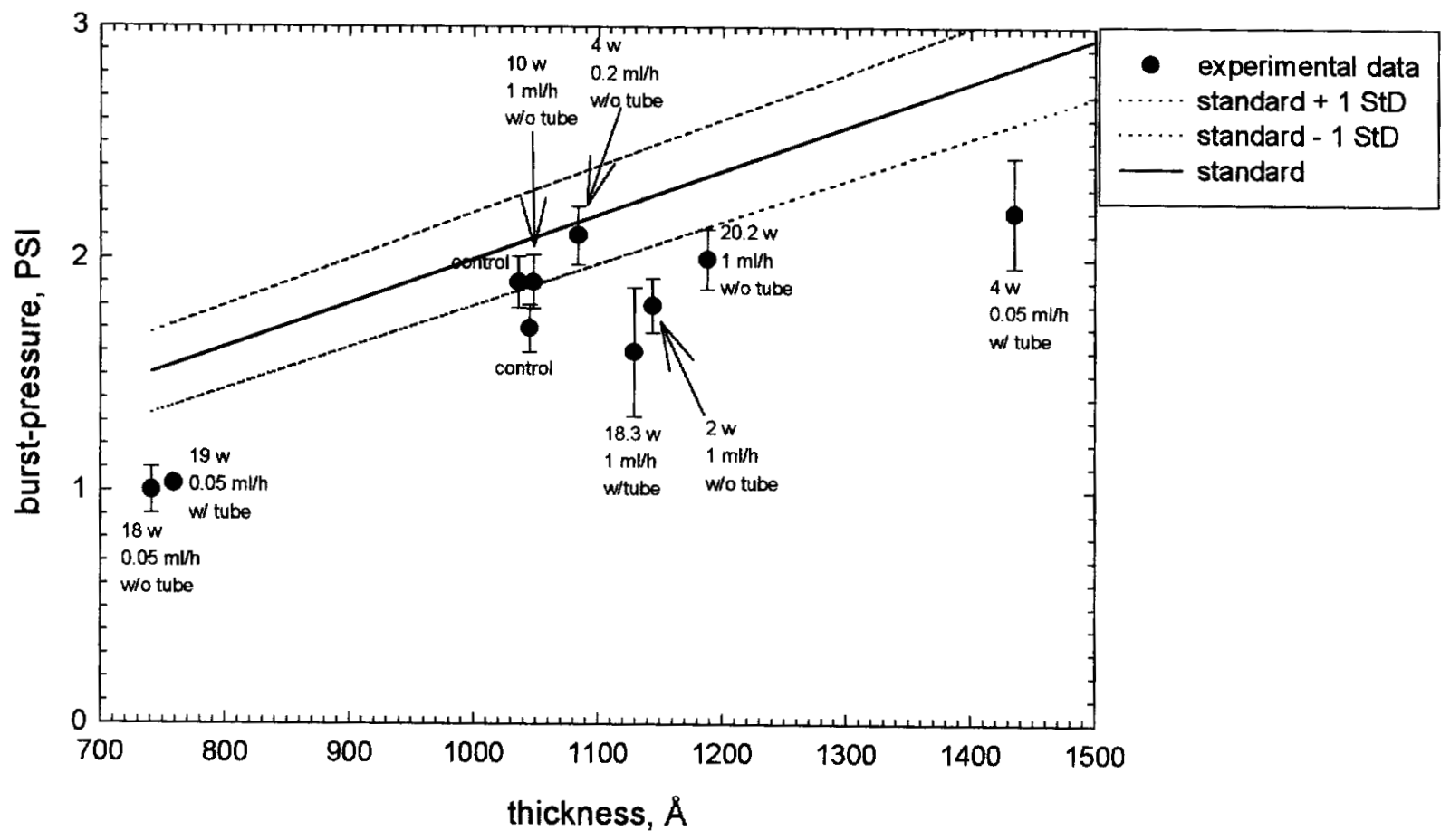




\section{Figure 4: Y-Hat Contour Plot of Power vs. Flow, Burst Test}

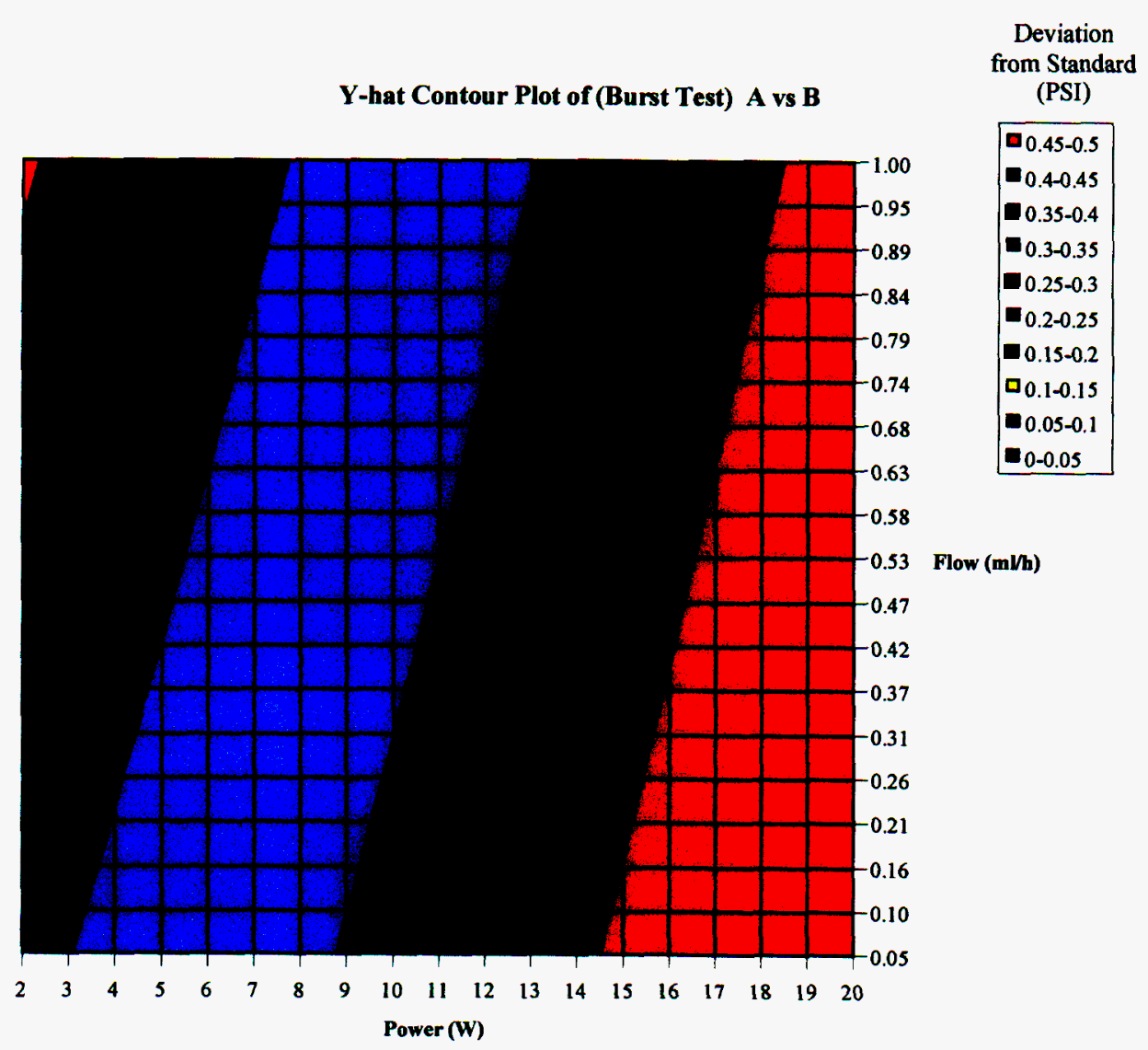

Figure 5

Y-hat Pareto of Coeffs - Burst Test

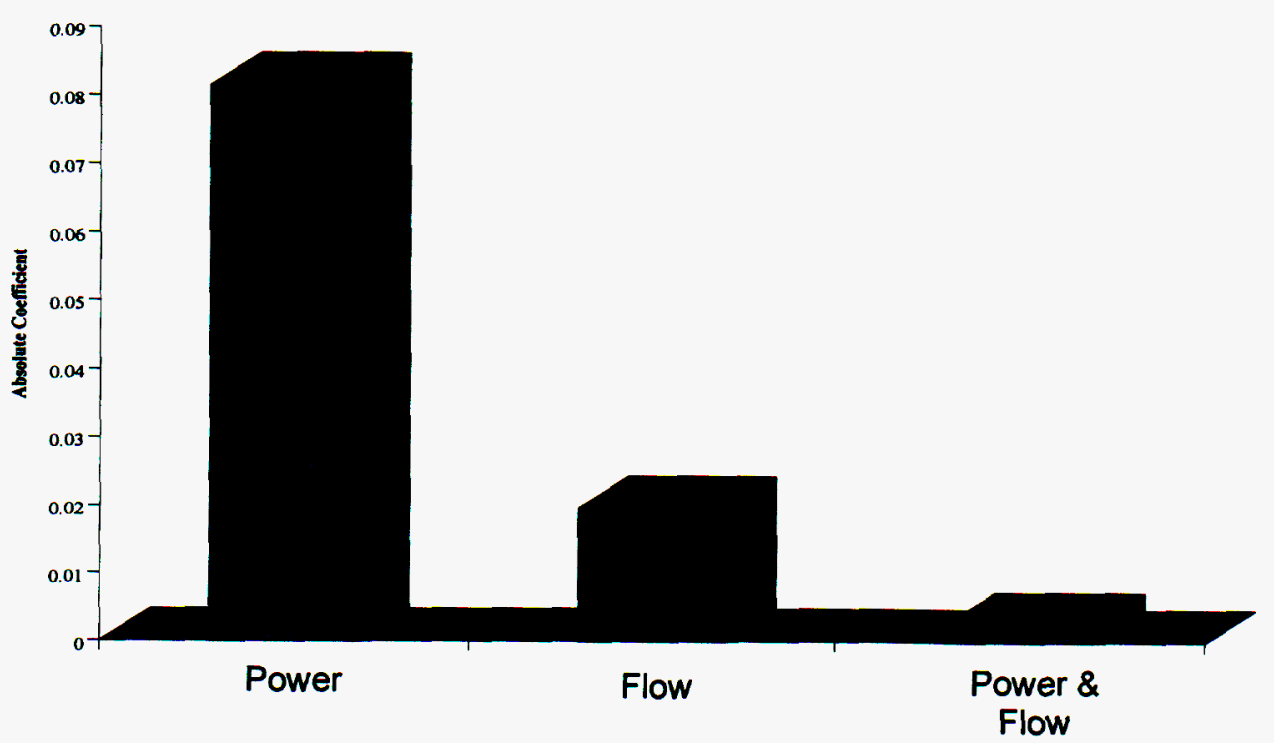


Figure 6

thickness Vs. polyamic acid flow-rate @ high atomization power

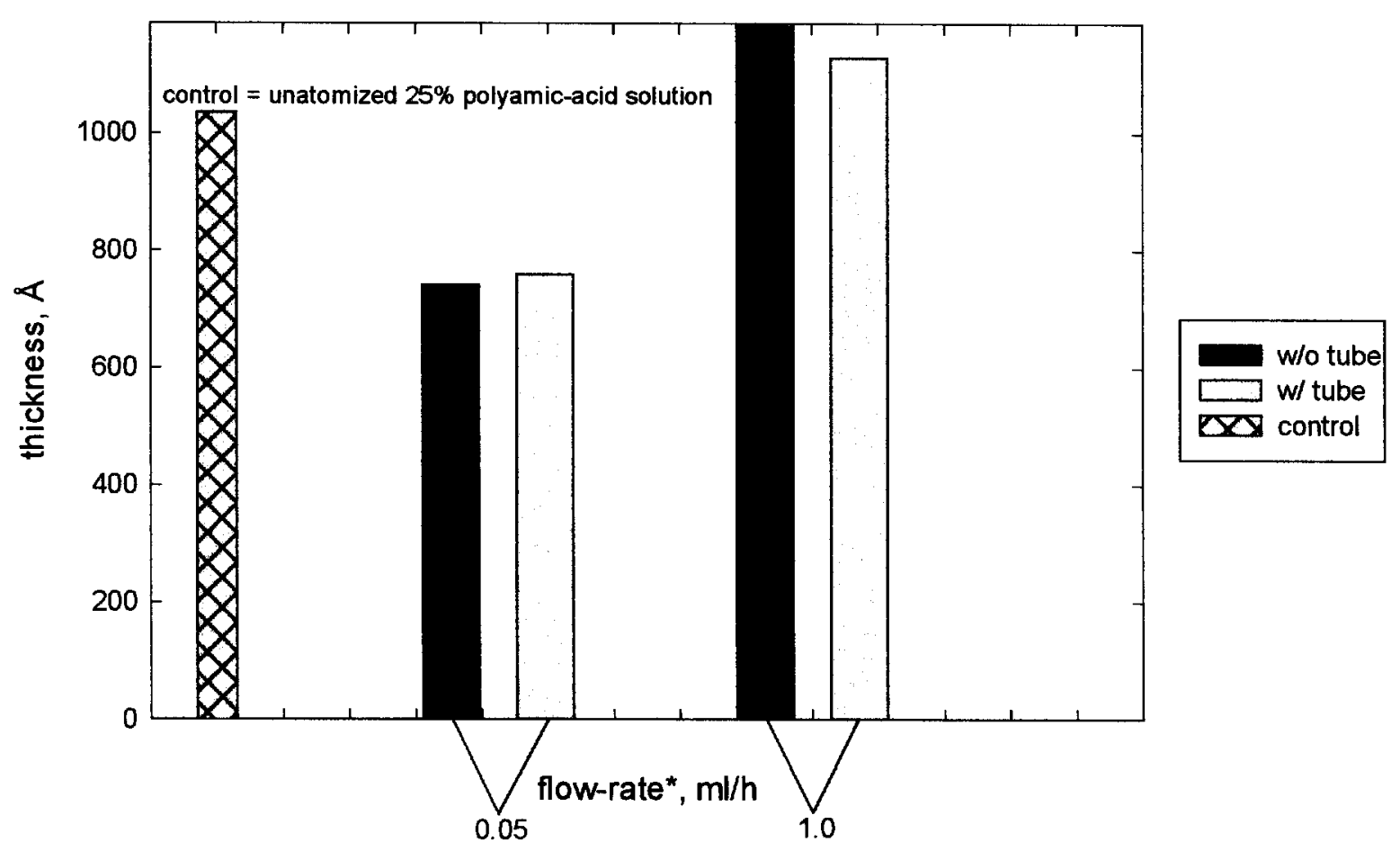


Figure 7a: Close-up of dry-spray area

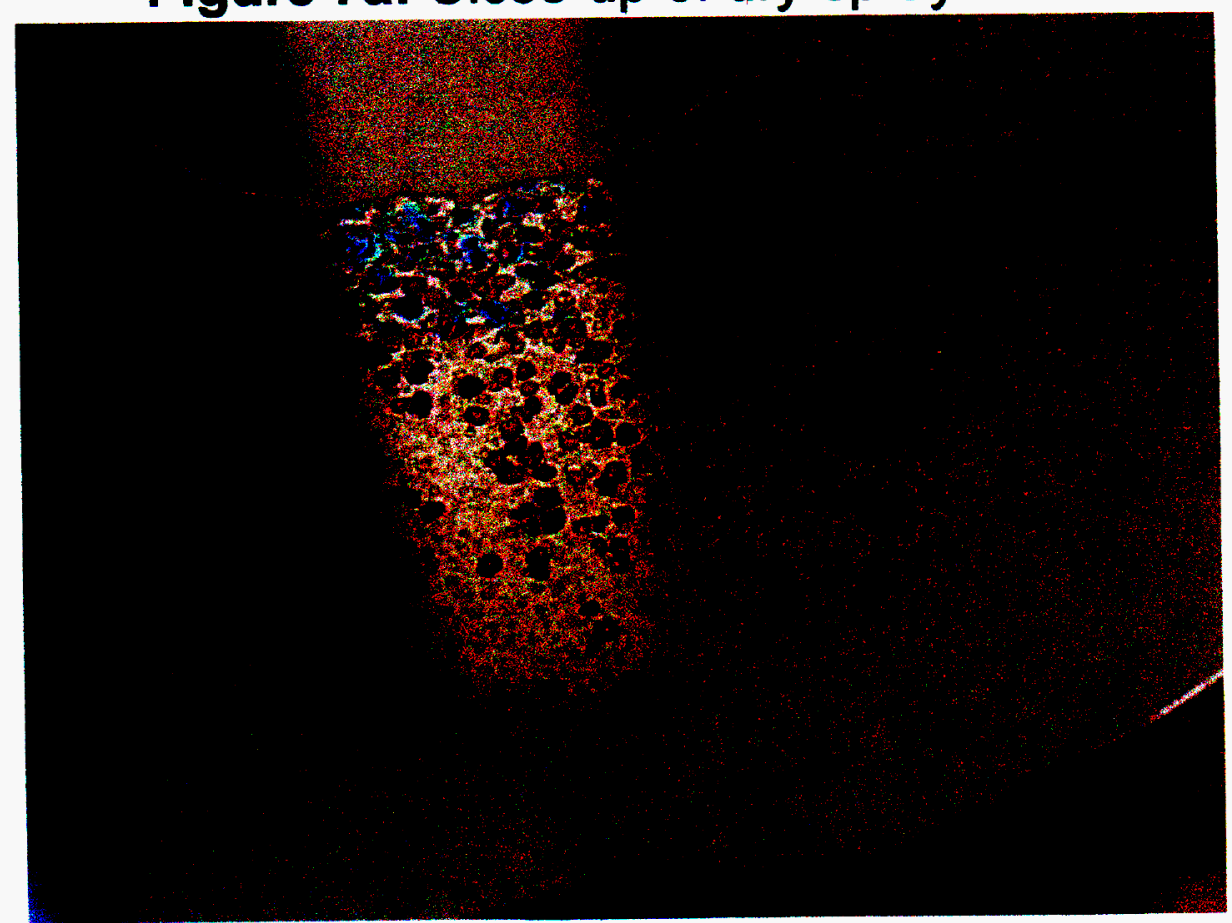

Figure 7b: Distribution of dry-spray area

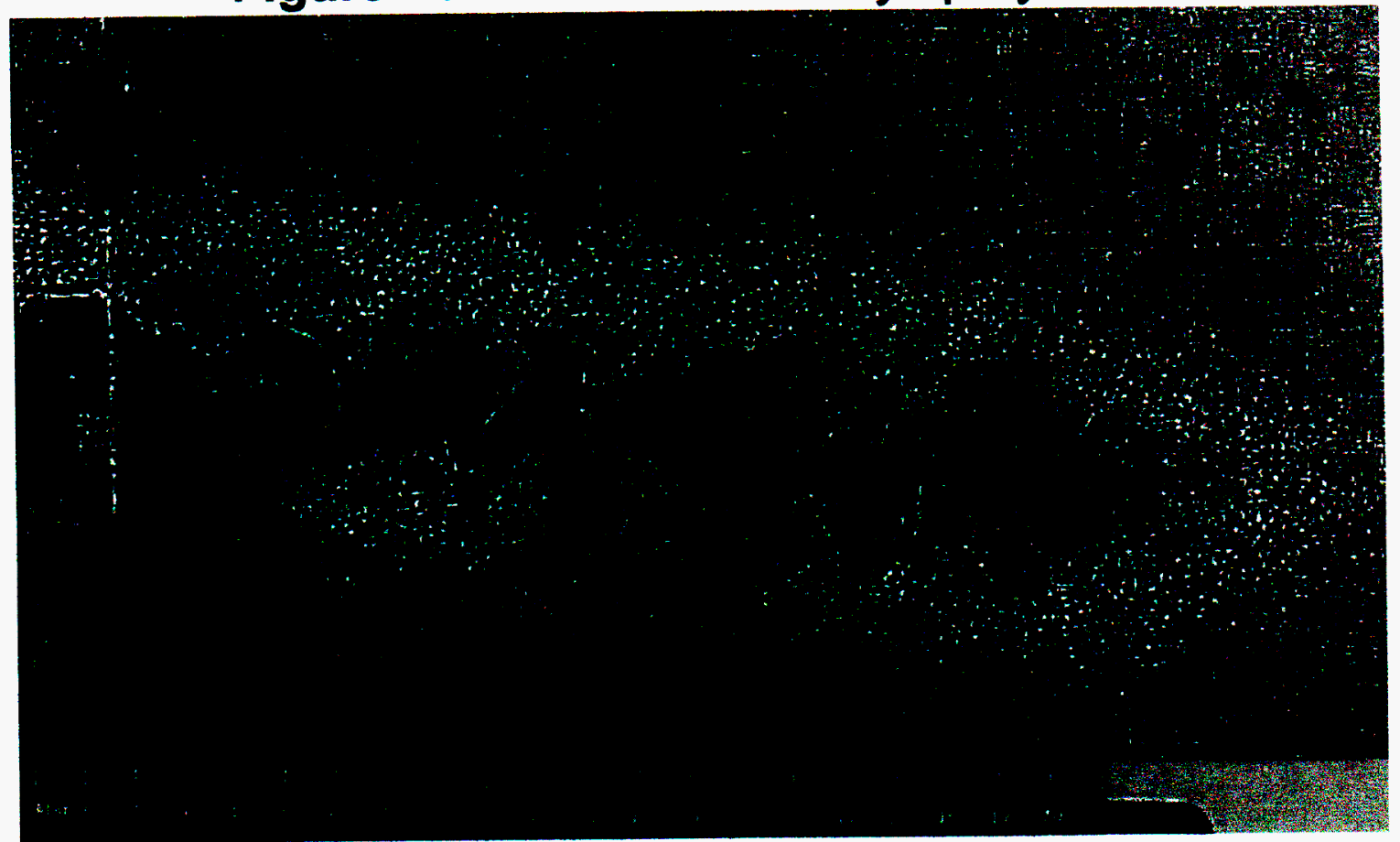


Figure 8: Brass mandral covered with PA

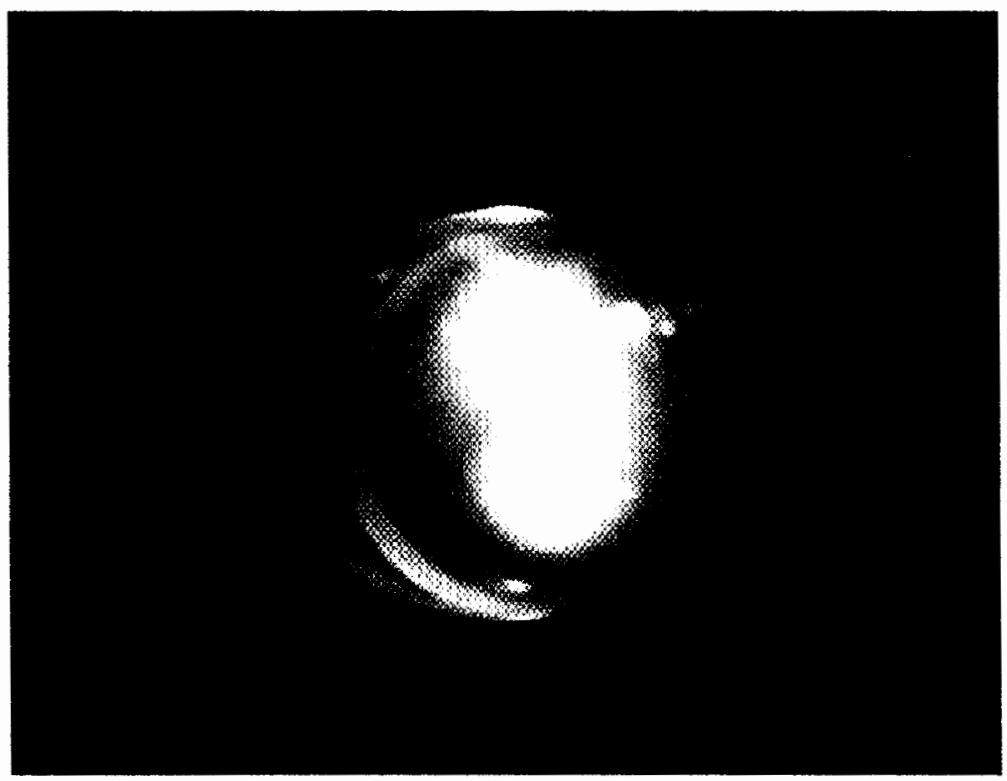

Figure 9: Brass mandrel after soft-baking

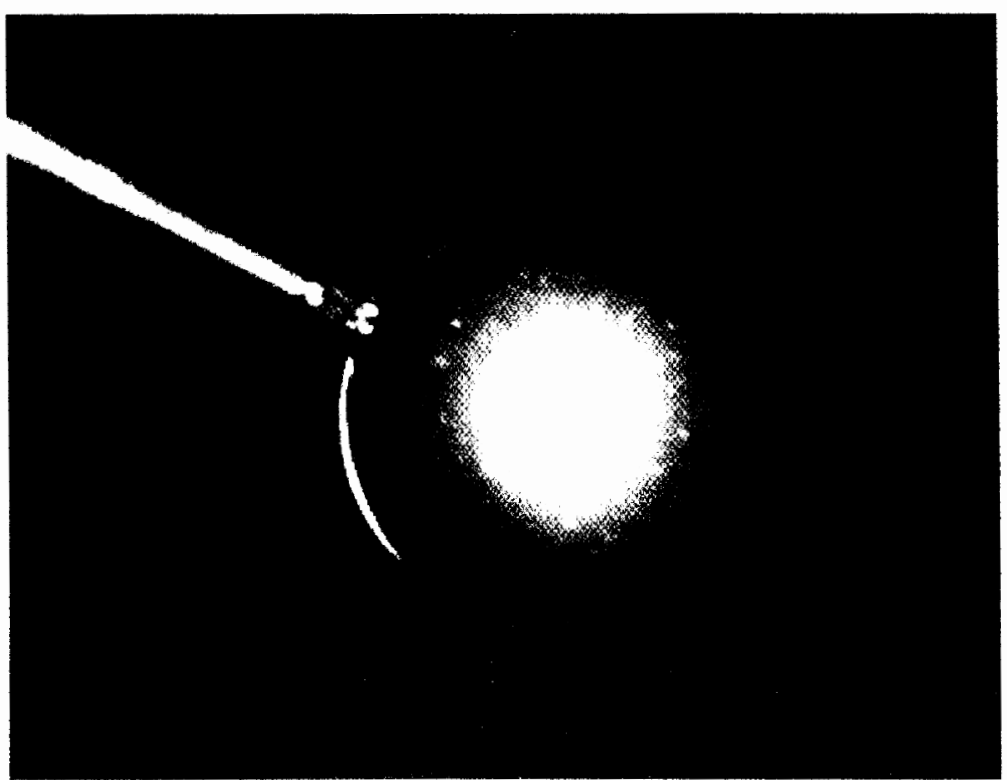


Figure 10: PA deposited on untreated $\mathrm{CH}$ mandrel by atomization

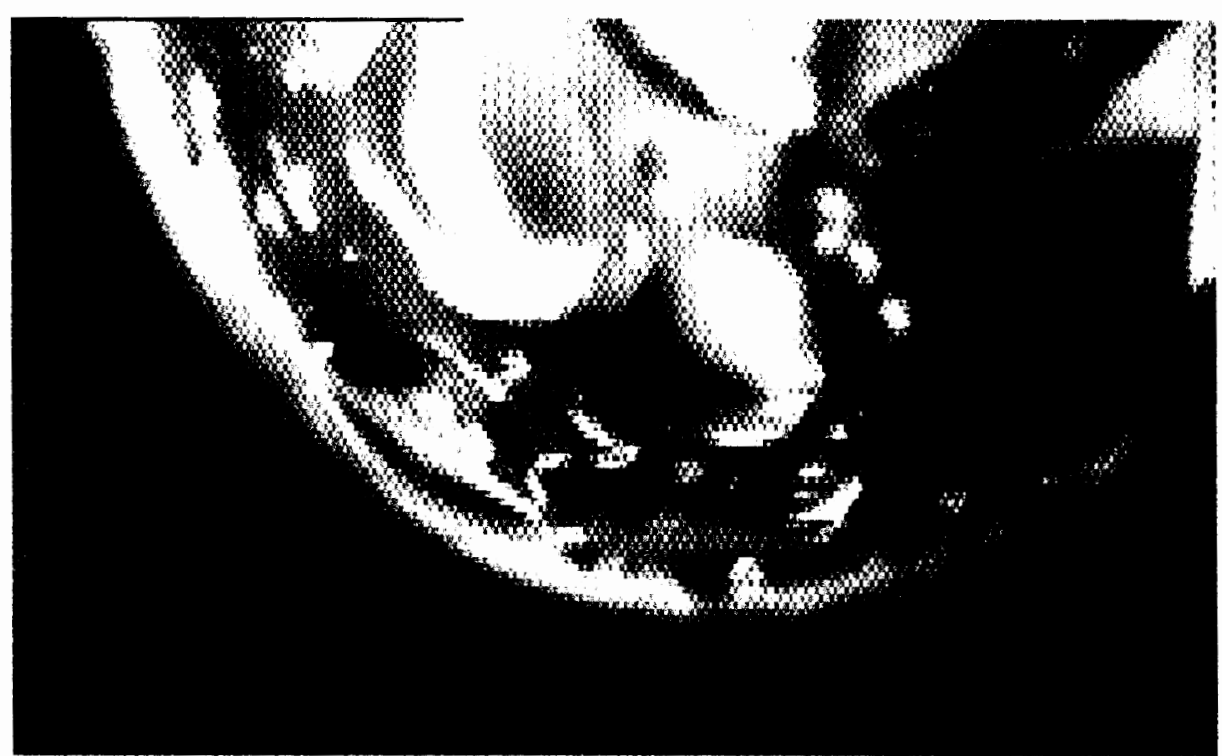


Figure 11a: $7.9 \mathrm{mg} 25 \%$ PA drop on spun, soft-baked NMP

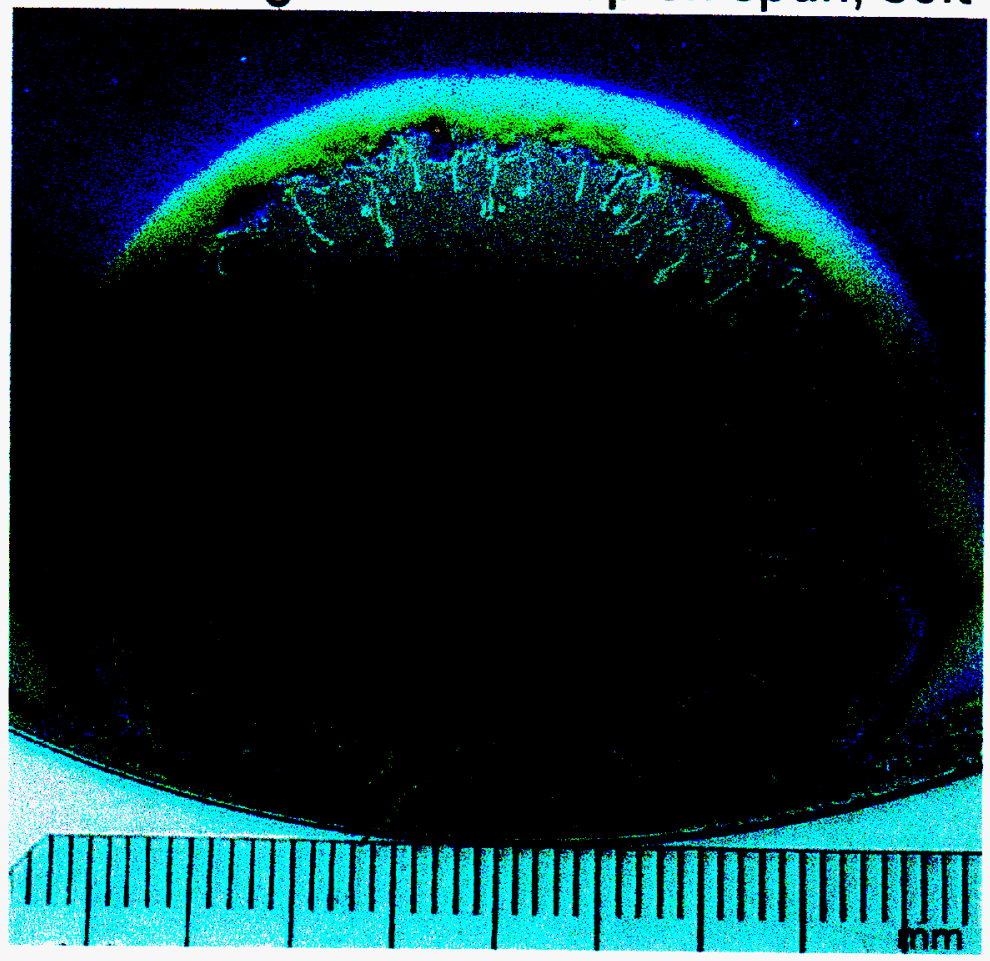

Figure $11 \mathrm{~b}$ : $7.5 \mathrm{mg} 25 \%$ PA on softbaked $25 \%$ P

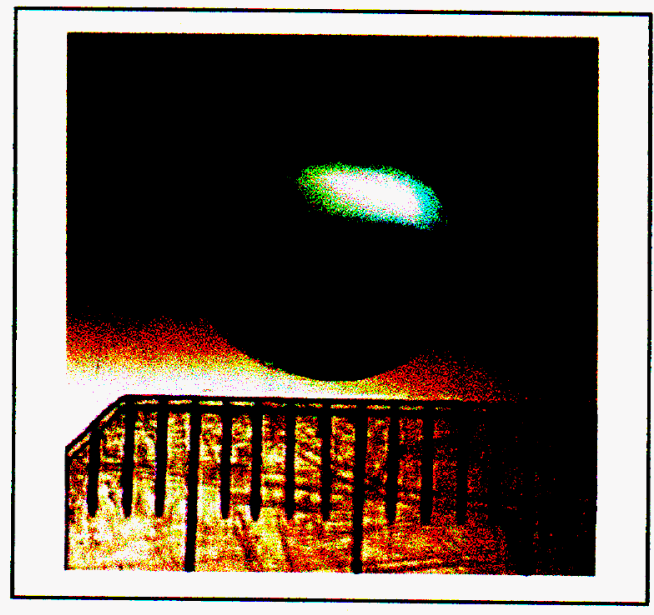


Figure 11c: $8 \mathrm{mg} 25 \%$ PA drop on imidized $P$ etched for 10 s in a $10 \%$ concentration $\mathrm{Hfl}: \mathrm{HNO}_{3}(1: 1)$

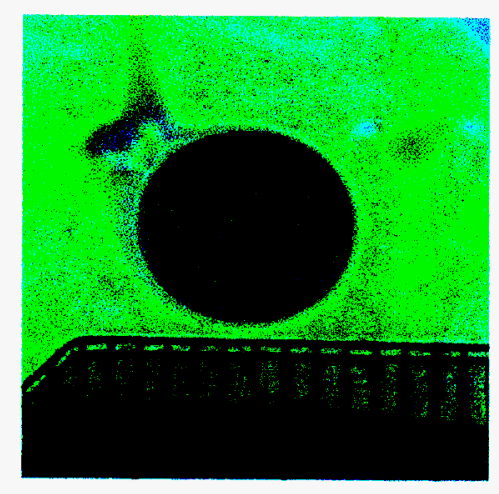

Figure 11d: $6.5 \mathrm{mg}$ PA drop on spun "Micro" cleaning/release agent

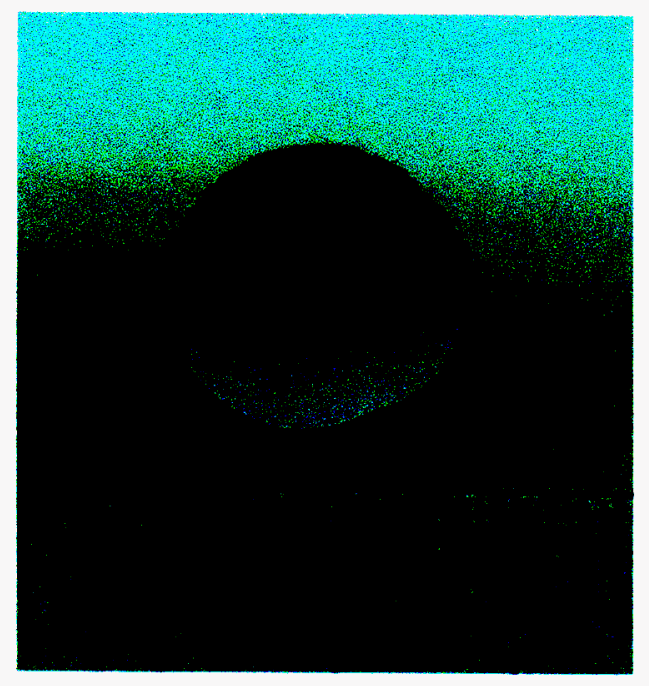

Figure 11e: $7.3 \mathrm{mg}$ PA drop on Ar ion etched $P$

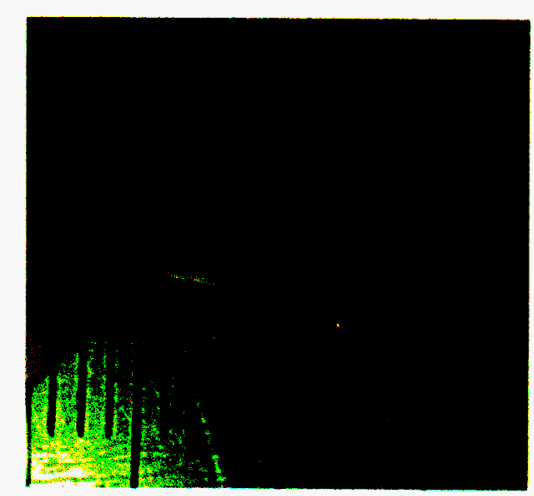


Figure 11f: PA Drop on Cascade (left) and clean Al substrate (right)

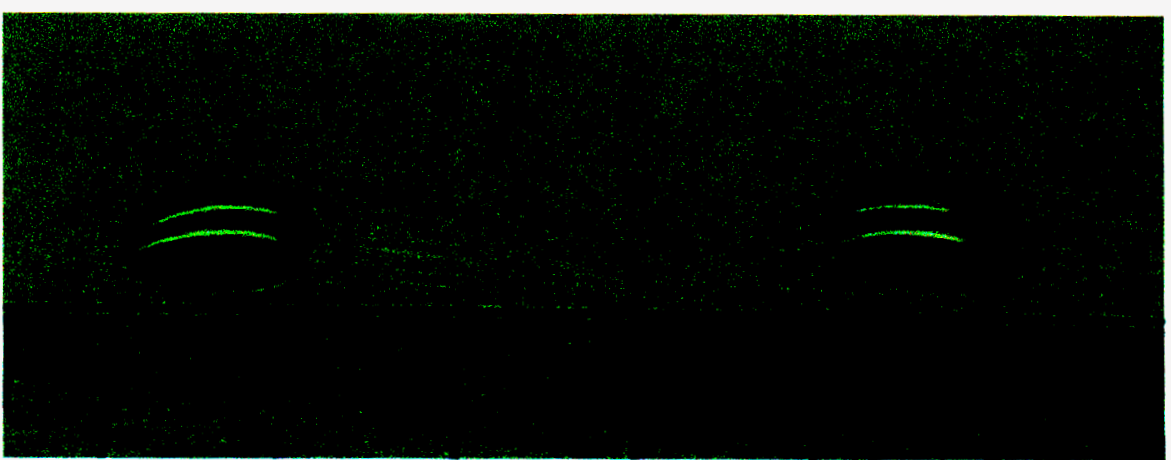




\section{Figure 12: NMP on untreated $\mathrm{CH}$ mandrel}

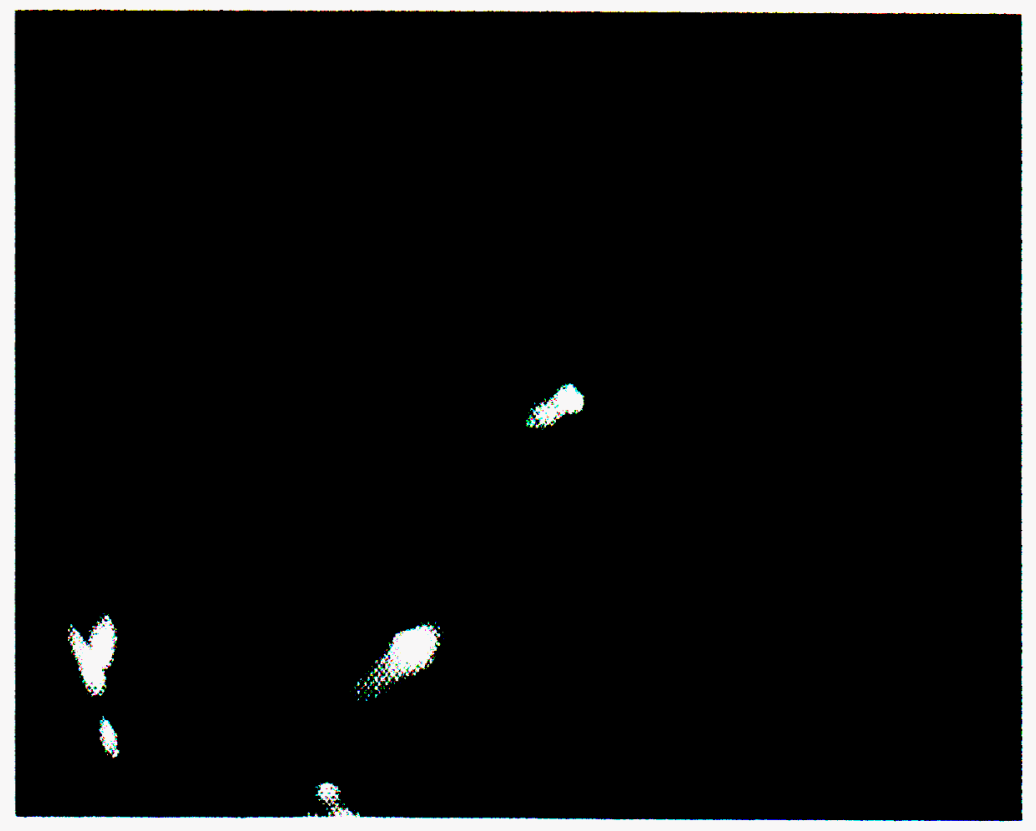

Figure 13: Droplets on inside surface of $\mathrm{CH}$ mandral at $50^{\circ} \mathrm{C}$

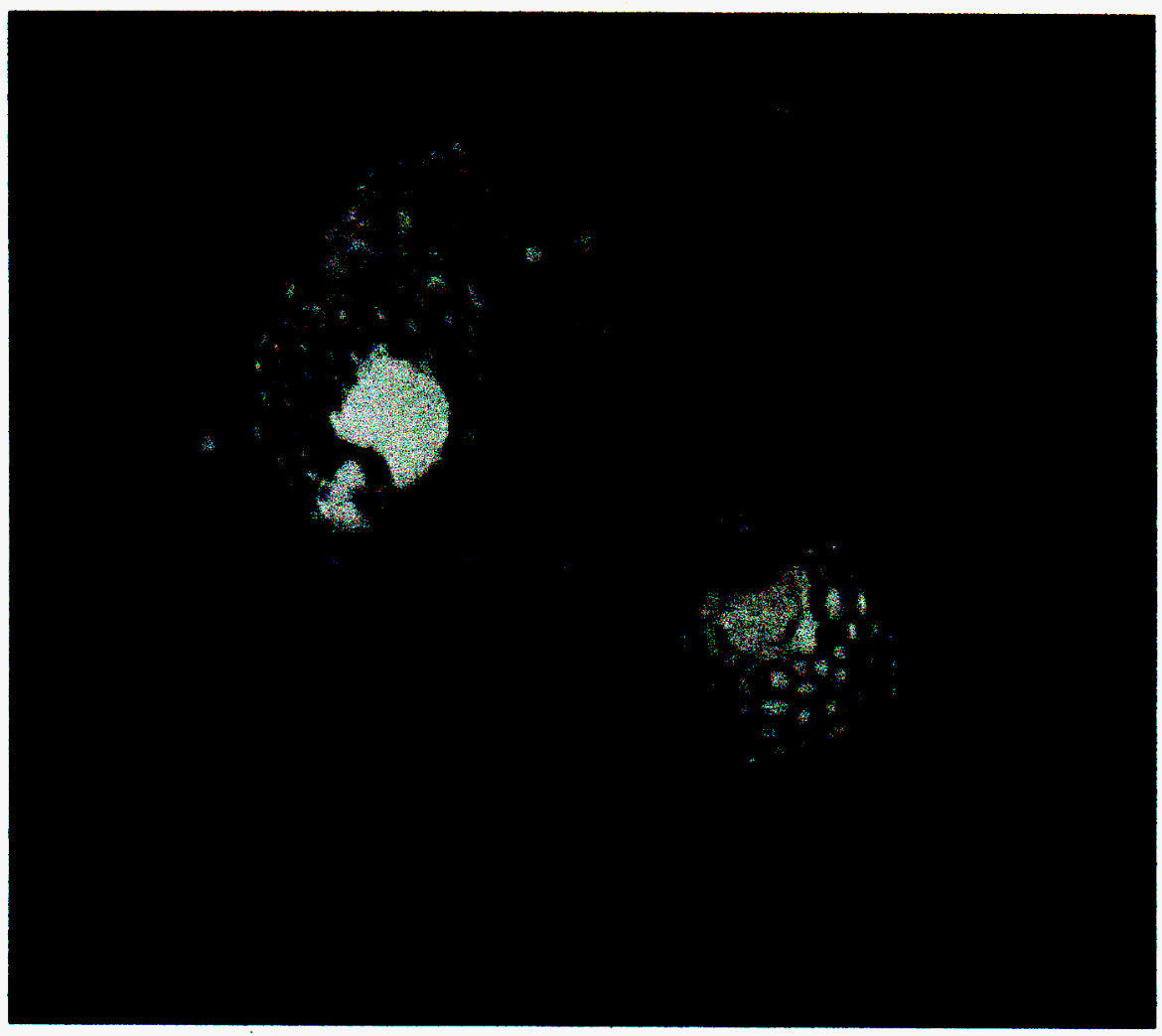




\section{Figure 14: Collapsed $\mathrm{CH}$ mandrel}

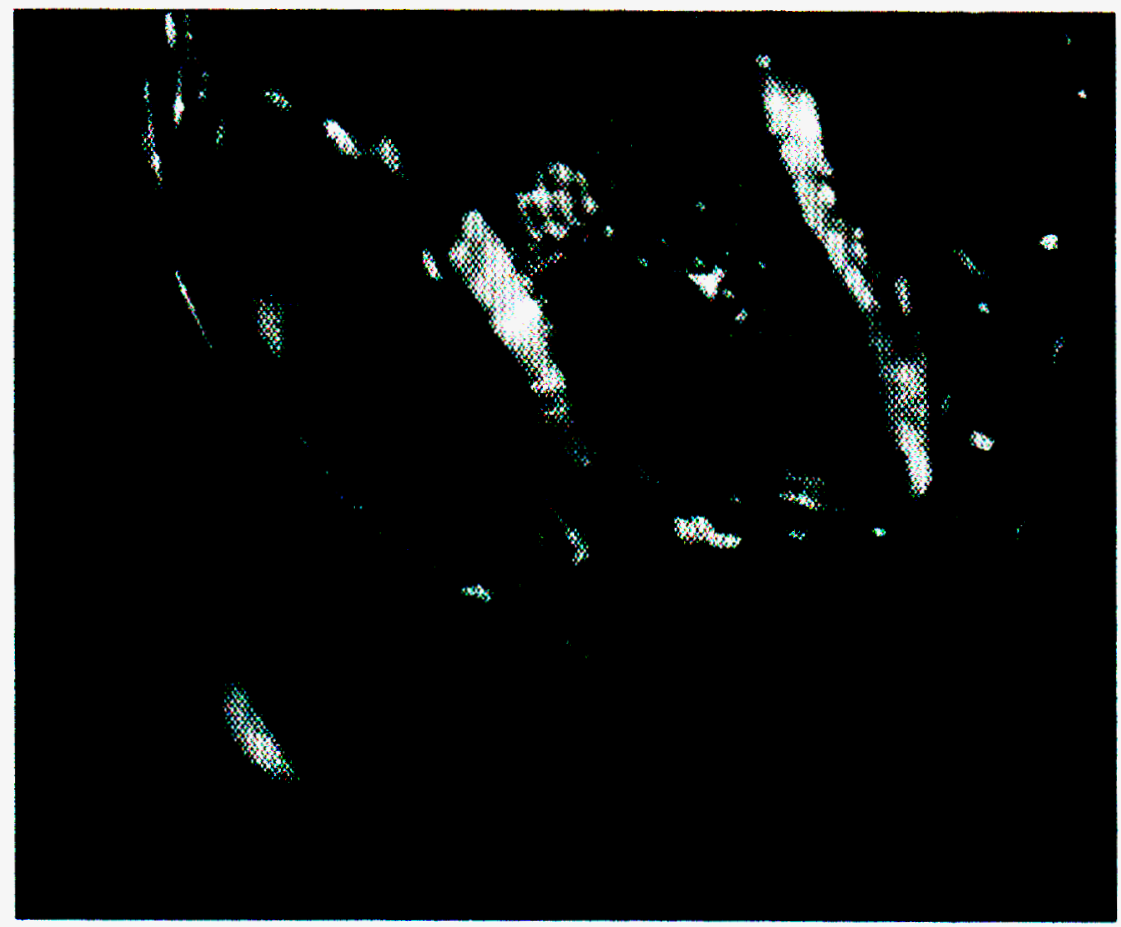

Figure 15: PA deposited onto oxygen plasma etched $\mathrm{CH}$ mandrel by atomization 
Figure 16: Atomized PA deposition onto air levitated $\mathrm{CH}$ mandrel

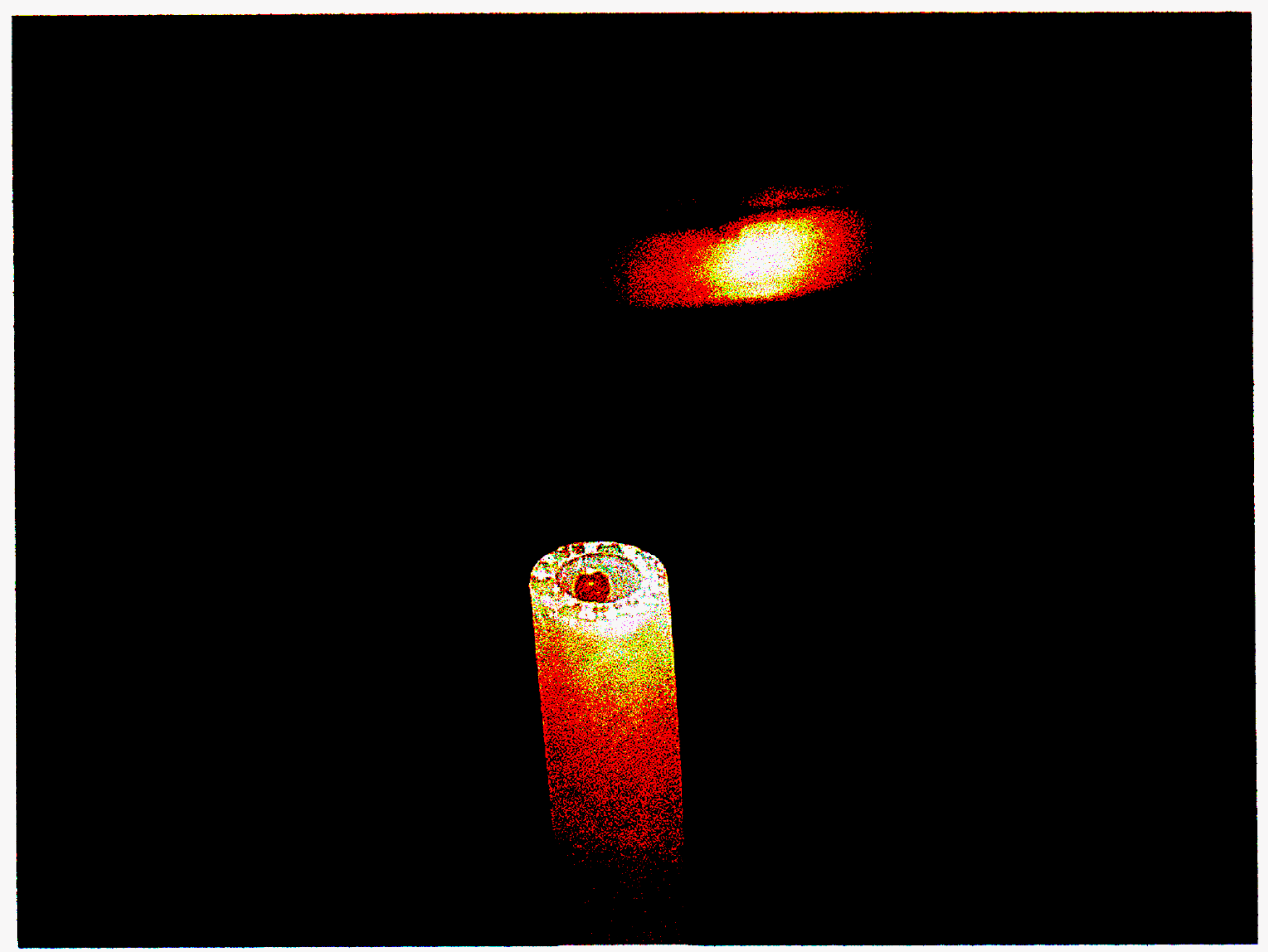




\section{Second Progress Report:}

\section{Investigation of Spray Conditions Necessary for Smooth Polyimide Coatings}

The previous progress report (5/15/2000) covering part one of the contract period investigated the spray conditions required to coat $\mathrm{CH}$-spheres with $\mathrm{P}$ (polyimide). The main conclusions of that report are:

1. Ultrasonic atomization is an appropriate means of dispensing PA (polyamic acid) if aggravated conditions of high atomization power ( 20 watts) and low PA flow rate are avoided.

2. PA dry spay is avoided if aggravated conditions of high air-flow rate through the atomizer and large radial distances $(>3 \mathrm{~cm})$ from the central atomized stream are avoided.

3. Dramatic improvement in the wettability of $\mathrm{PA}$ on $\mathrm{CH}$-sphere material is observed when using oxygen-etched $\mathrm{CH}$-spheres.

4. Manifestations of static buildup during atomized deposition of PA onto gas-levitated $\mathrm{CH}$ spheres have not been observed.

The following concerns were identified and investigated during the second part of the contract period to further our efforts in smoothly coating $\mathrm{CH}$-spheres with $\mathrm{P}$ :

1. Poor wetting significantly hinders formation of a smooth initial PA coat on $\mathrm{CH}$ spheres. Given this situation, we have investigated enhancing wettability in two different wetting scenarios: a) the $1^{\text {st }}$ PA coating where enhanced $\mathrm{CH}$ wettability is needed and b) subsequent $P A$ re-coats where enhanced soft-baked $P$ wettability is needed. Related to part $b$ is the possible dependence of wettability on NMP solvent lost during soft-baking.

2. Minimizing the amount of deposited PA to reduce slumping (due to gravity) while maintaining a smooth film. Related to this is optimizing the rotation rate required for a smooth, minimally slumped $\mathrm{P}$ film.

\section{Enhancing Wettability}

Examples of wetting $\mathrm{CH}$-spheres with PA are shown in Figs 1 and 2. Fig. 1 indicates that untreated PA does not readily wet an untreated $\mathrm{CH}$-sphere. However, by modifying a $\mathrm{CH}$ sphere's surface by oxygen etching, wettability is dramatically enhanced in Fig. 2 . These observations motived the following wetting experiments.

Results from the following soft-baking and wetting experiments are shown in Table 1. A 25\%PA solution was spun at 1000 RPM onto 5" silicon wafers. Samples 6 and 7 also include the surfactant (3M FC431) at $0.5 \%$ by weight. The wafers and the spun solution were weighed before and after soft-baking on a hot plate for different times at different temperatures. 
Standard volume drops $(7 \pm 1 \mathrm{mg})$, with and without surfactant, were then placed on the films and their diameters measured in order to get a rough idea of wettability.(Fig. 3 ).

The results indicate that NMP evaporates at surprisingly low temperatures in a short amount of time. We expected that wettability would be a function of mass loss, but this is not indicated. There appears to be some loss of wettability (with no increase in mass loss) going from $60^{\circ} \mathrm{C}$ to $125^{\circ} \mathrm{C}$. These encouraging results indicate that we can soft-bake at a relatively low temperature $\left(60^{\circ} \mathrm{C}\right)$ and remove most of the solvent without worrying about wettability. This should be readily attainable using the heat lamp

The surfactant actually decreased wettability at the lower temperature soft-bake and offered only a small amount of improvement at higher temperatures. The surfactant seems only useful on imidized $\mathbf{P}$ (Fig. 4a-b), which is a condition we will probably never have to face. It did improve wettability on an untreated $\mathrm{CH}$ sphere (Fig. 5), but not as much as the oxygen plasma etched sphere (Fig. 2). For comparison, non-surfactant PA on an untreated $\mathrm{CH}$ mandrel is shown in Fig. 1. We also tested the oxygen plasma etched Kapton sample for wettability (Fig. 6). The non-surfactant PA seemed to wet this material better than the surfactant PA. The surfactant only improved wettability significantly on sample 7 , a film containing surfactant that had only a $76 \%$ mass loss. We therefore conclude that the surfactant offers no observable advantage in the time and temperature regime of interest.

Given these encouraging experimental results on wettability, we returned to depositing atomized PA onto gas-levitated $\mathrm{CH}$-spheres. A number of following issues become evident during this effort:

1) Full atomization deposition coverage of the air-levitating mandrel is possible. The levitating mandrel appeared to rotate in a random fashion as it was hit by droplets. Also, more heavily coated sides of the mandrel rotated down under the effect of gravity as we deposited from above, contributing to full coverage.

2) PA with surfactant will cover untreated $\mathrm{CH}$ similar to PA without surfactant on oxygen etched $\mathrm{CH}$. Compare Figs. 7 and 10 to compare fully covered mandrels. Compare Figs. 5, 9, and 1 to see how different solutions and surface treatments wet.

3) A smooth surface (as indicated by perfect looking reflections) appeared only after a large amount of PA was deposited. The deposited PA droplets coalesced only to a point and the PA drop material did not seem to move or slump very much. They did not smooth out over the surface of the mandrel until long after the mandrel appeared fully coated. This may be good for limiting slumping of PA and large scale non-uniformity, but is not good for higher mode smoothness because at present, it is difficult to maintain air levitation stability for very long periods ( $\geq 2$ hours) while depositing. The sample shown in Fig. 13 when compared to figure 8 (which has a greater amount of PA deposited) shows that we can perhaps improve higher mode uniformity by depositing more PA before soft-baking. Fig. 14 is similar to Fig. 13 in that it is under coated. Figs. 10 and 11 are the same mandrel except Fig. 11 was taken after soft-baking with a second coat started, after the mandrel fell out of levitation. The large 
difference in appearance between figures 10 and 11 may be due in part, to differences in imaging and not soft-baking and/or the unfinished second coating. Back lighting can reveal the surface roughness as shown in figure 15.

4) It is possible to apply a smooth looking film over a rough looking one. Figure 12 shows a partially re-coated mandrel where the thicker second coat appears smooth.

\section{Minimizing deposited PA to reduce slumping, while maintaining a smooth film}

The motivation for the following series of experiments is to study conditions necessary for nonslumped, smooth-layering of soft-baked polyimide onto a surface that is similar to that encountered when spray-coating PA onto a $\mathrm{CH}$ sphere. The approach uses vertically rotating disks that are spray coated with PA using a stationary atomizer.

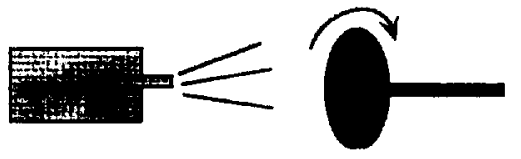

We think our results will correlate to a levitating $2 \mathrm{~mm}$ diameter sphere being spray coated in the following manner. An extreme situation occurs at the equator of a $\mathrm{CH}$-sphere, where gravity and flow are in the same direction. Vertically mounted samples sprayed on from the side reflect this case. The more manageable case of a rotating disc is analogous to the spherical mandrel rotating around a single axis. Our results will at least give us a relative answer as to which deposition conditions minimize slump.

Initial Vertical Spray Experiments

Our initial vertical spray experiments are beginning to reveal the relationship between the amount of $\mathrm{P}$ deposition, the speed of rotation and uniformity, both large scale (dripping or slumping) and small scale $(\sim 100 \mu \mathrm{m}$ - due to individual drops). The images, surface profile scans, and 1-D power spectra (Figs. 16 and 17), all depict the buildup and flowing together of individual droplets, moving from outside to the center of the sprayed area where they transition to a relatively smooth, $\sim 100 \AA \mathrm{rms}$ film at $\sim 3500 \AA$ in thickness. These results seem to indicate that individual droplets do not influence surface roughness after this transition thickness has been reached. Slumping does occur but it can be reduced by rotating the sample.

SETUP: $5^{\prime \prime}$ silicon wafers coated with approximately $1400 \AA$ soft-baked, spun-cast $P$ were mounted vertically on a rotating fixture. $25 \%$ PA flowing at $0.05 \mathrm{ml} / \mathrm{h}$, was sprayed from the atomizer, set at $2 \mathrm{~W}$ of power, $71 \mathrm{~mm}$ away. This distance was chosen to obtain a diffuse spray pattern at the 1 SCFH airflow rate we typically use. After spraying, the samples were soft-baked using an unfocused heat lamp while still rotating. A rough calibration of this heat source was made by irradiating a $30 \mathrm{~mm} \times 30 \mathrm{~mm} \times 0.127 \mathrm{~mm}$ Kapton sample at different voltage settings (Fig. 18). The temperature of the Kapton was monitored using an infrared probe. At each setting, the lamp was turned off when the temperature stopped rising. The temperature was recorded once it stopped falling, indicating that uniform bulk temperature of the Kapton had 
been reached. This calibration was meant to estimate the heating of a levitating $\mathrm{CH}$ mandrel, which is difficult to measure directly. It probably underestimates the heating of the mandrels (lower mass) and over-estimates the heating of wafers. We reported earlier that NMP is removed at relatively low temperatures and soft-bake temperature has no appreciable effect on wetting until somewhere over $100^{\circ} \mathrm{C}$ - so we are not overly concerned with accurate soft-bake temperature control.

\section{RESULTS and DISCUSSION of Initial Vertical Spray Experiments}

ROUGHNESS: The stationary sample (Fig. 16) transitioned to a relatively smooth surface at about $2500 \AA$ thickness and the sample rotated at 3 RPM (Fig. 17) at about $4000 \AA$. We're not sure yet if the discrepancy in thickness is real and due to the rotation or to inaccurate measurements (one vs. four averaged data points). This amount of variability in atomization rate is not unusual in our experience. Most of the activity in the power spectra occurs at $\sim 100 \mu \mathrm{m}$, approximately corresponding to the size of atomized PA droplets, where there is roughly three orders of magnitude difference in intensity between the center where the surface is smooth, and the outside edge where there are discrete single droplets. The stationary sample had a RMS surface roughness of $372 \AA$ and the rotating sample $127 \AA$ in the center including perhaps, some of the $109 \AA$ rms substrate as shown in Fig.19. This is close to the $91 \AA$ NIF specification. These RMS values came from a $4000 \mu \mathrm{m}$ scan, corresponding to mode 1.6 on a $2 \mathrm{~mm}$ mandrel. Our roughness corresponds favorably to the $490 \AA$ mode 2 RMS reported by Cook et al. ${ }^{1}$-although mode number in our flat substrate experiments effectively corresponds to our largest features, the "slumps", where we are no where near the NIF specification. Figures 20 and 21 show surface roughness versus. position and thickness.

ROTATION: A large, $19 \mu \mathrm{m}$ drop formed on the stationary sample. The center of the sample rotated at $3 \mathrm{RPM}$ also slumped, forming a $1 \mu \mathrm{m}$ ridge. These experiments indicate that slumping is definitely something we need to be concerned with. The over-riding question we need to answer is if it is possible to deposit the relatively large amount of PA apparently necessary for higher mode smoothness and minimize low mode "slumping." Next, we plan to increase rotation rate in the hope that this will improve low mode uniformity even further, as is already indicated by the improvement we have seen by rotating at only 3 RPM.

The acoustic levitator has been used to levitate $\mathrm{CH}$ mandrels, providing some experience with this technique. We also applied a vacuum above the air-levitating mandrel using our vacuum chuck according the technique used by Kim et al. ${ }^{2}$, but observed little improvement. The geometry of our setup is different than theirs and is probably the reason we saw little of the advantages they reported.

1 R.C. Cook, R.L. McEachern, R.B.Stephens "Representative Surface Profile Power Spectra from Capsules Used in NOVA and OMEGA Implosion Experiments"

2 K. Kim, Q Feng "A Novel Gas-Dynamic Levitation Scheme for Non-Contact Coating of Spherical ICF Targets" Fusion Technology, 28, 95 


\section{Current Vertical Spray Experiments}

Similar to the initial spray experiments, we continued to investigate the effect of rotation rate on smoothness and slumping. Paremeters for the samples considered here are listed in Table 2 Several issues have become evident:

1. Variability in the rate of PA deposited by the atomizer is significant if it is stopped and then restarted. This can be seen in Figs. 22 and 23 where we briefly (< $2 \mathrm{~min}$ ) stopped spraying to replenish its PA supply. The spray pattern became significantly larger after restarting. To compare a series of spayed, rotating disks in terms of slumping and smoothness as function of time and/or rotation rate, the atomizer must run continuously. Samples 18 and 19 (Figs 25 and 26 ) are another example of atomizer variability. Even though PA flow rate is reduced by a factor of 2 , the RPM rate increased by a factor of $\sim 1.3$, and the PA deposition time reduced by a factor of 1.3 between samples 18 and 19, the central thickness still increased by a factor of 1.3 .

2. All of our vertically sprayed samples (Figs 24 to 26) indicate that a smoothness transition is accompanied by slumping, regardless of rotation rate. Thickness values in the smooth areas range between 3300 and $7200 \AA$, RMS roughness values range between $\sim 90$ and $\sim 540 \AA$ and slump heights range between $\sim 3.1$ and $\sim 13.1 \mu \mathrm{m}$. The smoothest sample had the largest slump: Sample 20, Fig. 26. These samples indicate that the smoothness transition occurs at a thickness of $\sim 3500 \AA$ with a RMS roughness transitioning from $\sim 500$ to $\sim 150 \AA$. This is approximately consistent with our initial vertical spray transition results. 


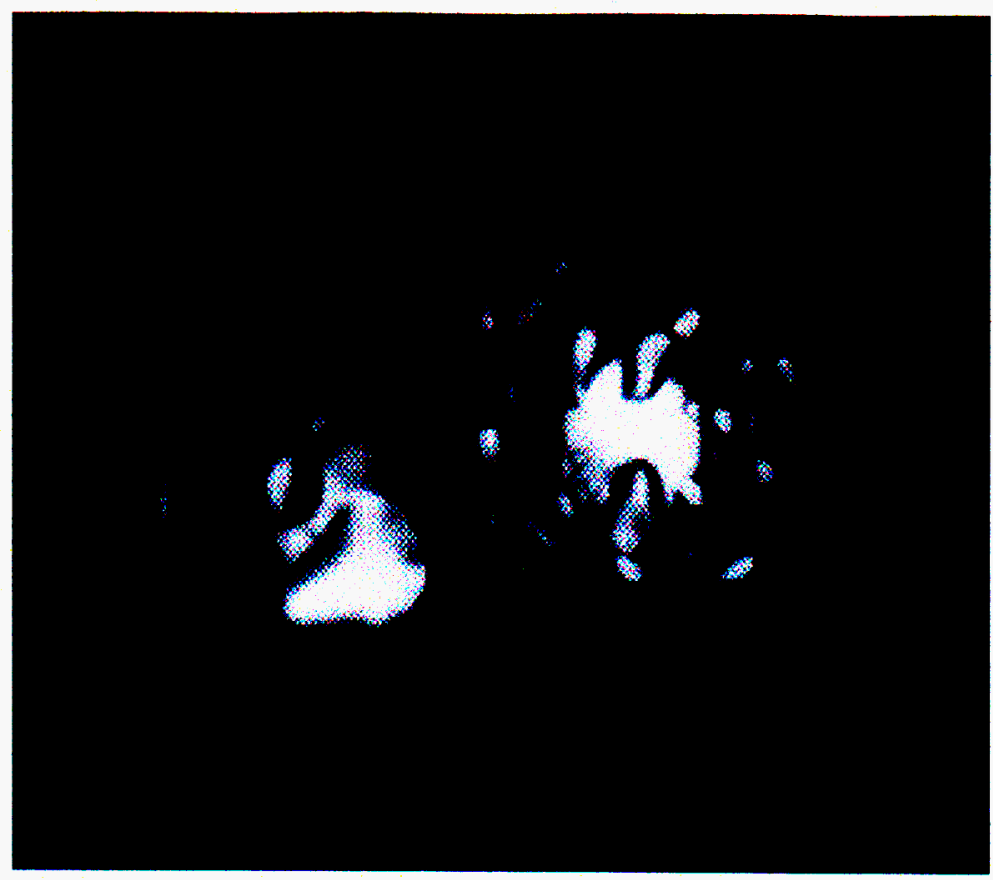

Figure 1: PA on untreated Levitating $\mathrm{CH}$ Mandrel

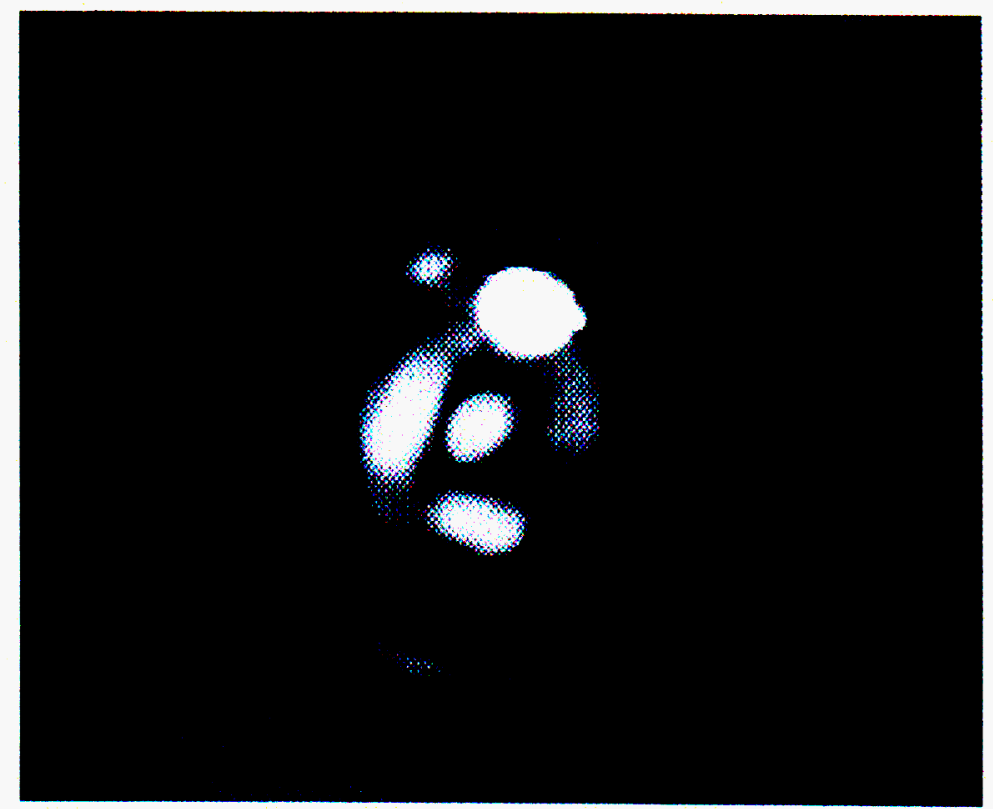

Figure 2: PA on Oxygen-Etched CH Mandrel 


\begin{tabular}{|c|c|c|c|c|c|}
\hline $\begin{array}{c}\text { Sample } \\
\#\end{array}$ & $\begin{array}{c}\text { Soft-bake } \\
\text { Temperat } \\
\text { ure ('C) }\end{array}$ & $\begin{array}{c}\text { Soft-bake } \\
\text { Time (s) }\end{array}$ & $\begin{array}{c}\text { \% Mass } \\
\text { Loss }\end{array}$ & $\begin{array}{c}\text { Diameter of } \\
\text { Equal Volume } \\
\text { Drops -No } \\
\text { Surfactant }\end{array}$ & $\begin{array}{c}\text { Diameter of } \\
\text { Equal Volume } \\
\text { Drops -.5\% } \\
\text { Surfactant }\end{array}$ \\
\hline 1 & 60 & 30 & 96 & 9.0 & 8.0 \\
\hline 2 & 65 & 300 & 97 & 9.0 & 8.5 \\
\hline 3 & 125 & 300 & 97 & 7.5 & 8.0 \\
\hline 4 & 32 & 300 & 75 & 8.0 & 8.0 \\
\hline 5 & 300 & 180 & 97 & 9.0 & 10.2 \\
\hline 6 & 36 & 300 & 96 & 7.0 & 7.0 \\
\hline 7 & 35 & 100 & 76 & 9.0 & 11.5 \\
\hline
\end{tabular}

Table 1

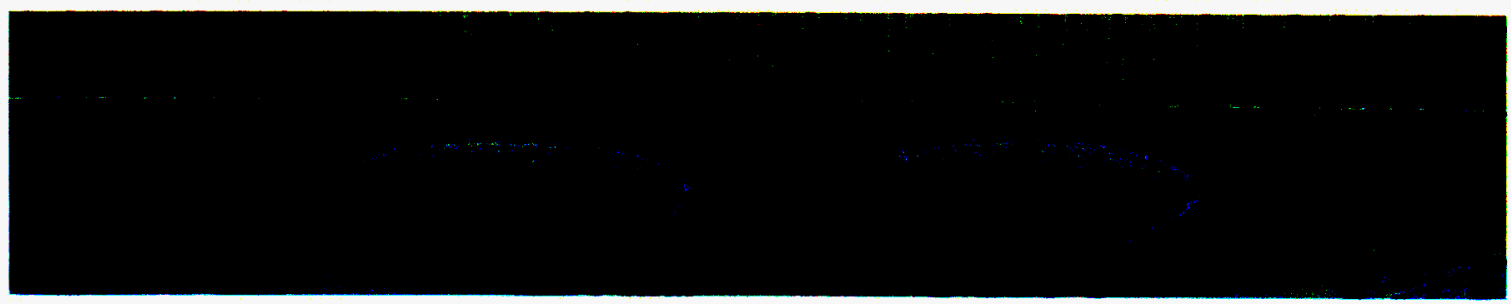

Figure 3: Measuring Drop Diameter (mm scale)

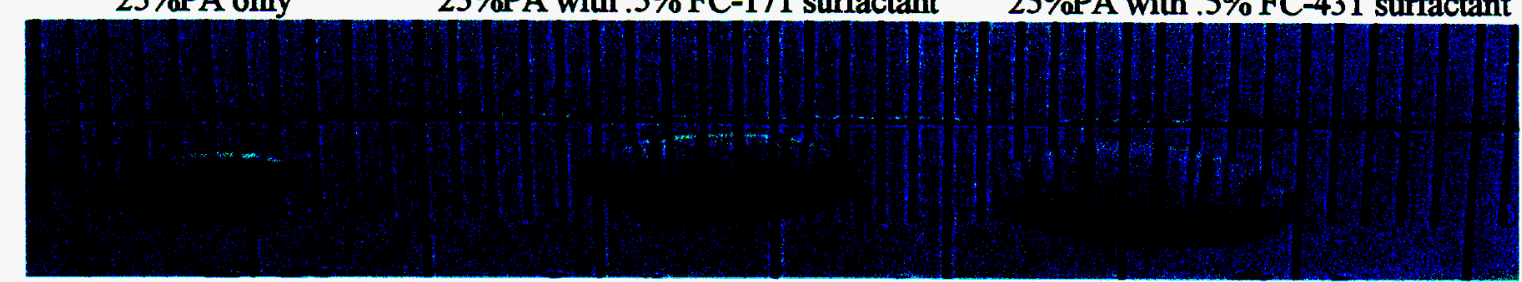

Figure 4a: Drop Diameter on Imidized P

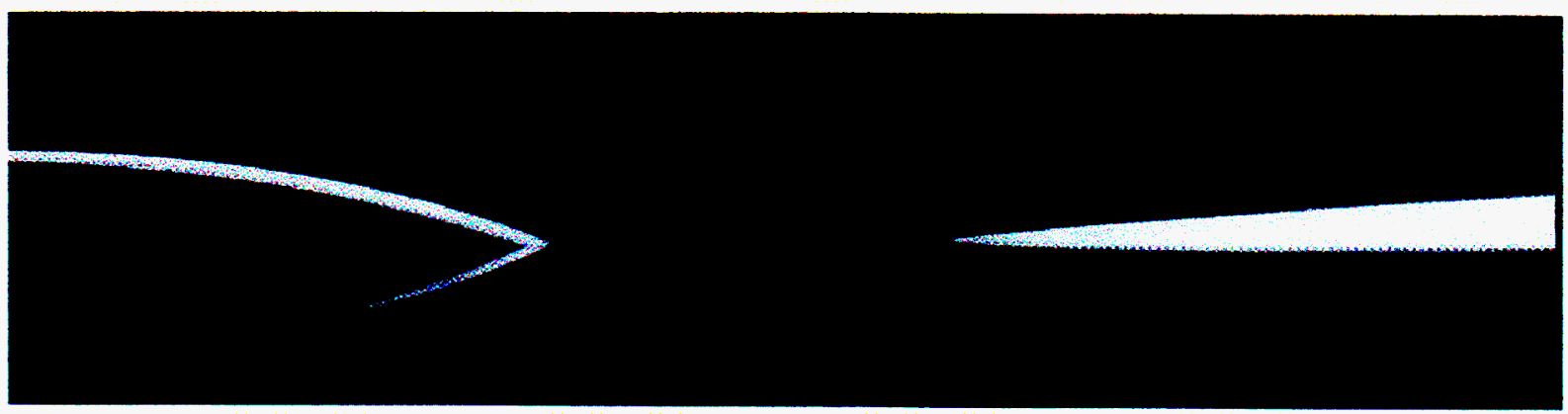

Figure 4b: Wetting Angle of 25\%PA (on left) and 25\%PA with Surfactant (right) on Imidized P 


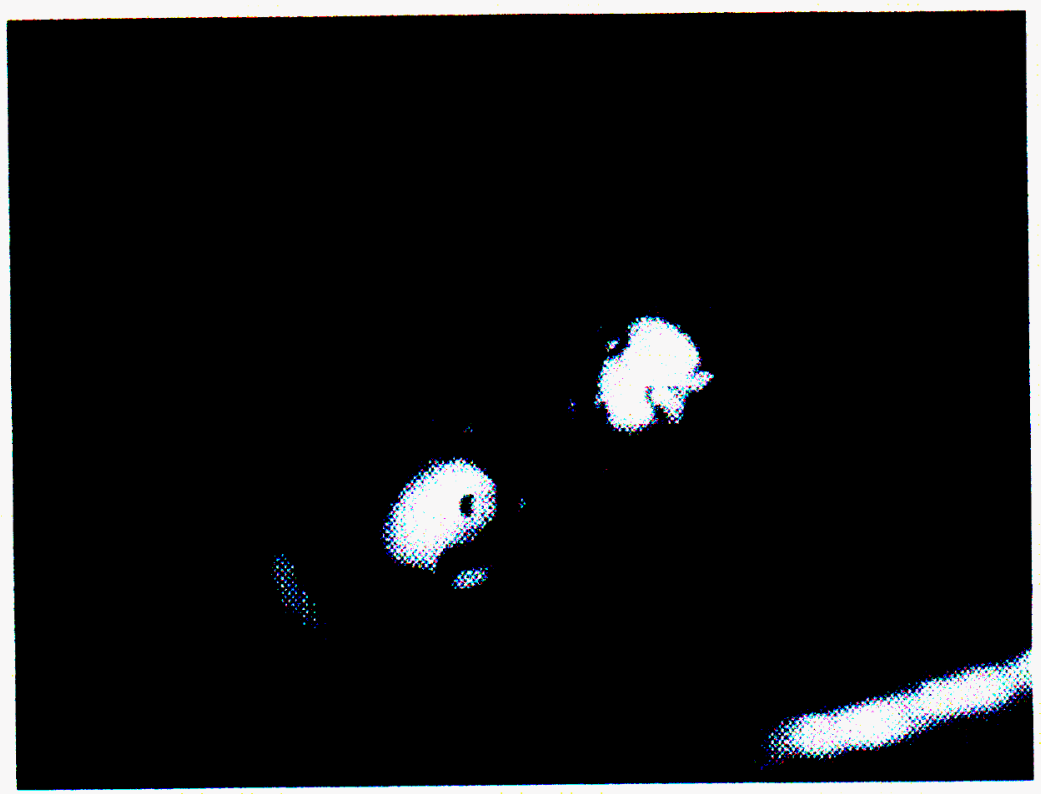

Figure 5: 25\%PA with .5\% FC-431 Surfactant Atomized onto Untreated CH Mandrel

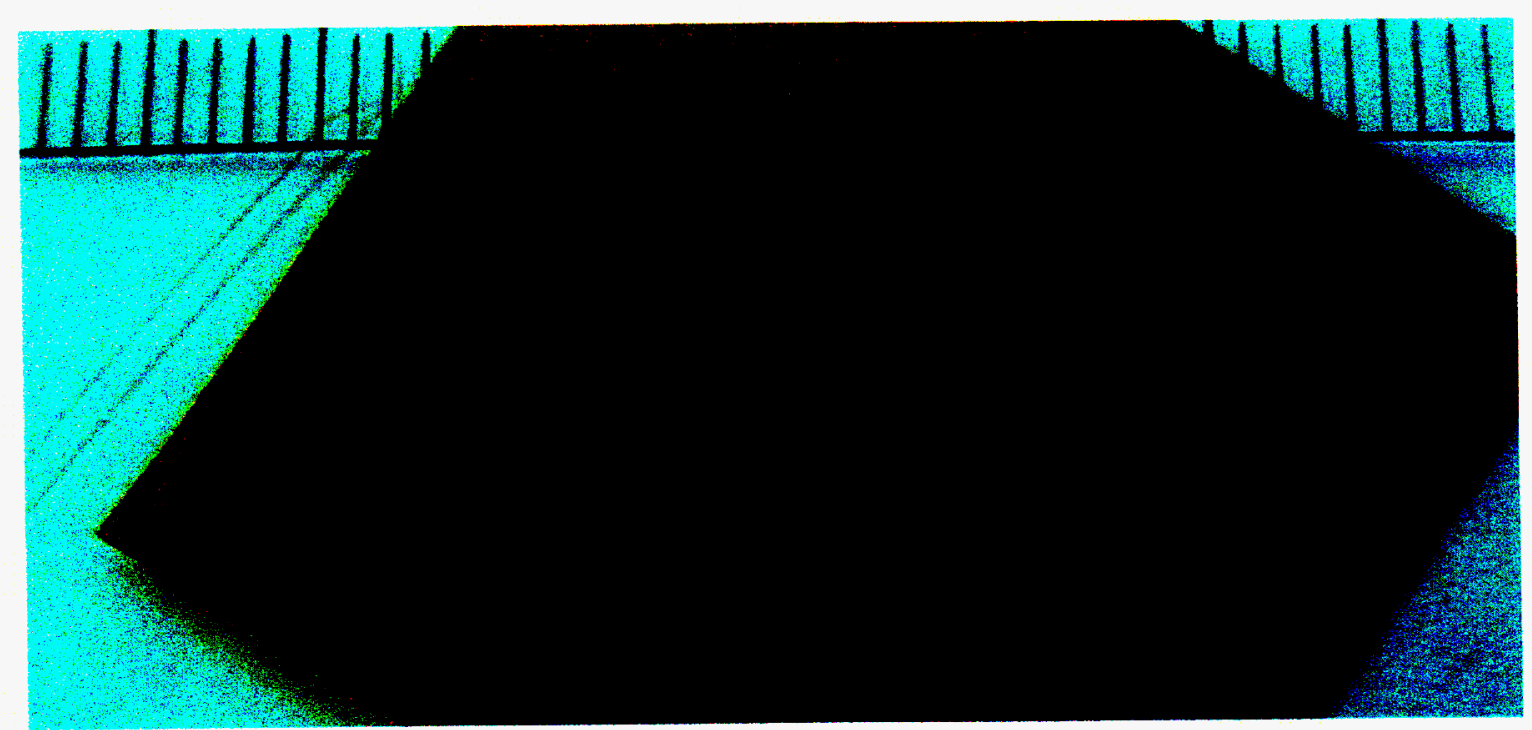

Figure 6: 25\%PA (on left) and 25\%PA with Surfactant (right) Drops on Oxygen Etched Kapton 


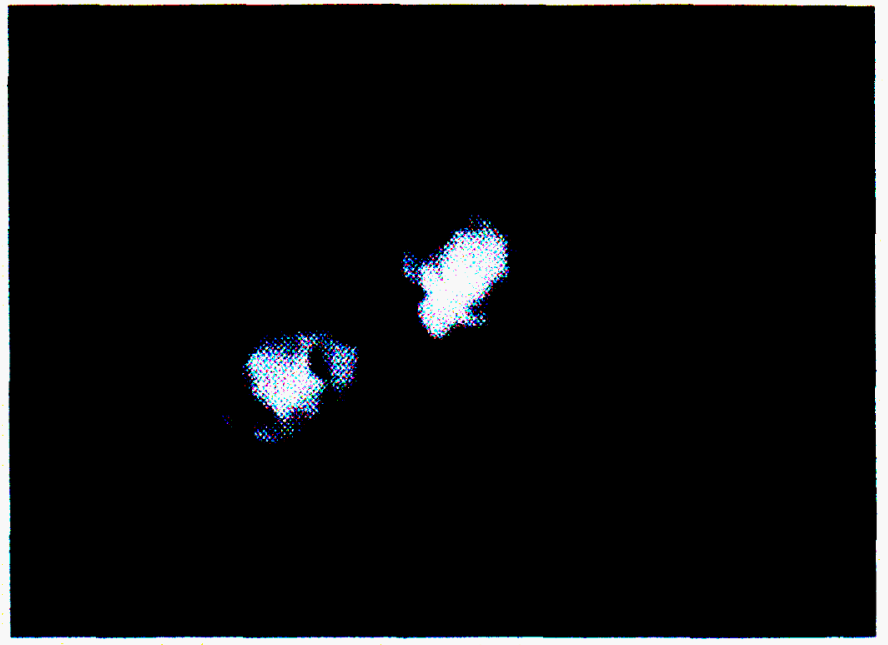

Figure 7: 25\%PA with .5\% Surfactant on Untreated Levitating CH Mandrel After Full Coat (sample \#1)

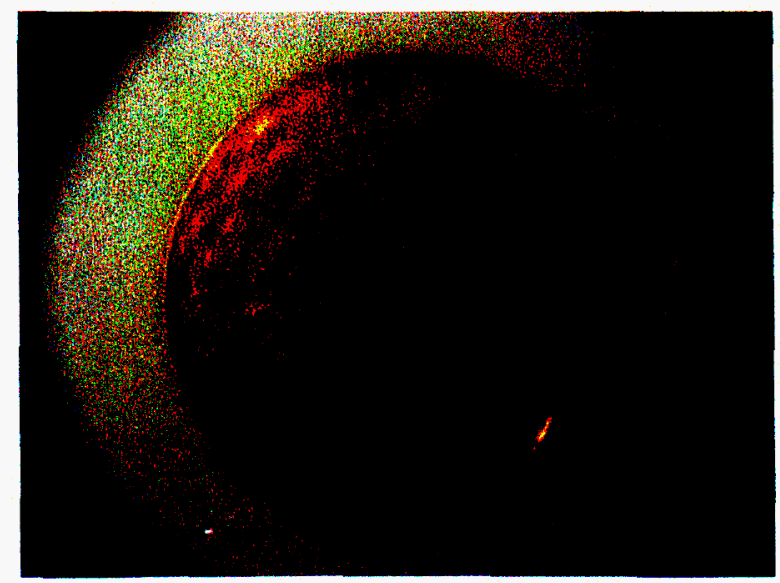

Figure 8: 25\% PA with .5\% Surfactant on CH Mandrel after Full Coat - Not Levitating (sample \#1)

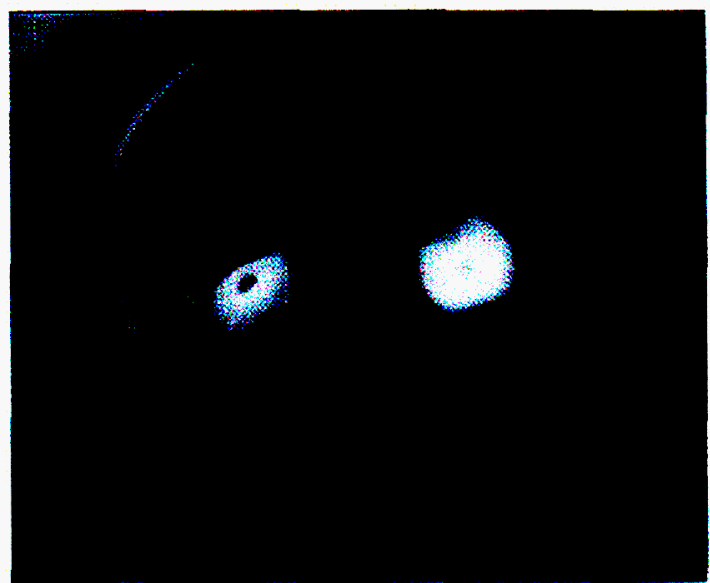

Figure 9: 25\%PA on Oxygen Etched, Levitating CH Mandrel Beginning of Coating (sample \#6) 


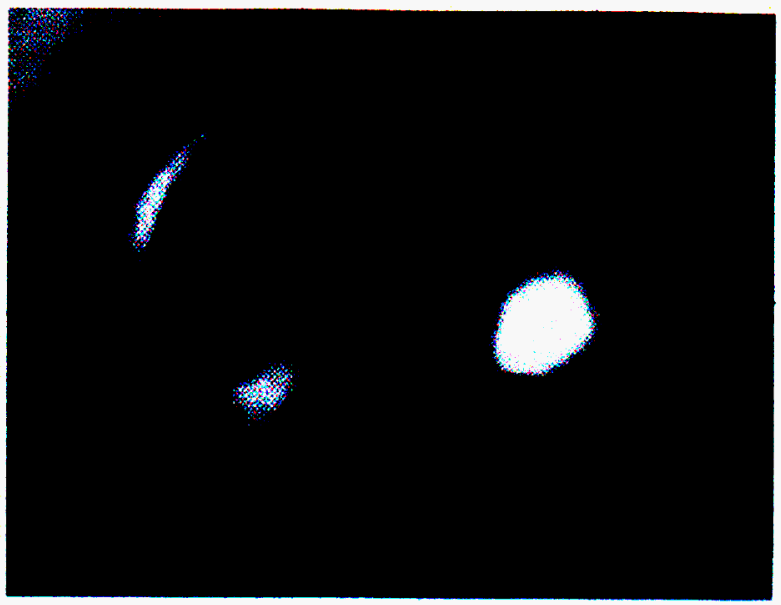

Figure 10: 25\%PA on Oxygen Etched, Levitating CH Mandrel after Full Coat and Before Soft-Bake (sample \#6)

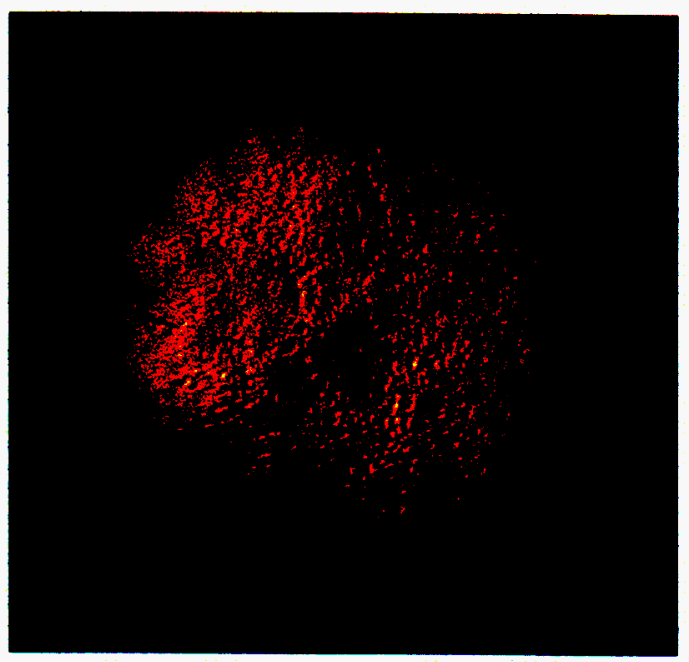

Figure 11: 25\%PA on Oxygen Etched CH Mandrel after Soft-Bake - Not Levitating (sample \#6)

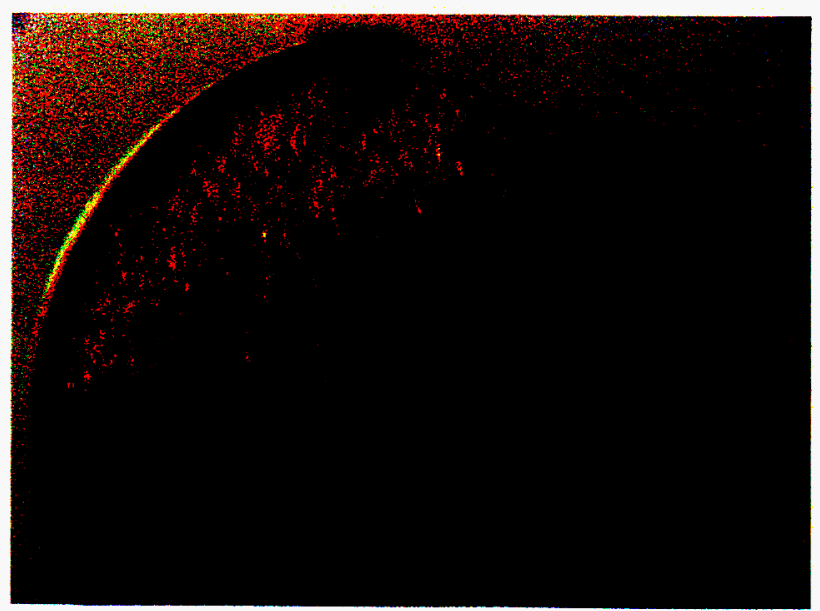

Figure 12: 25\%PA on Oxygen Etched CH Mandrel after Soft-Bake and Partially Into 2nd Coating (sample \#6) 


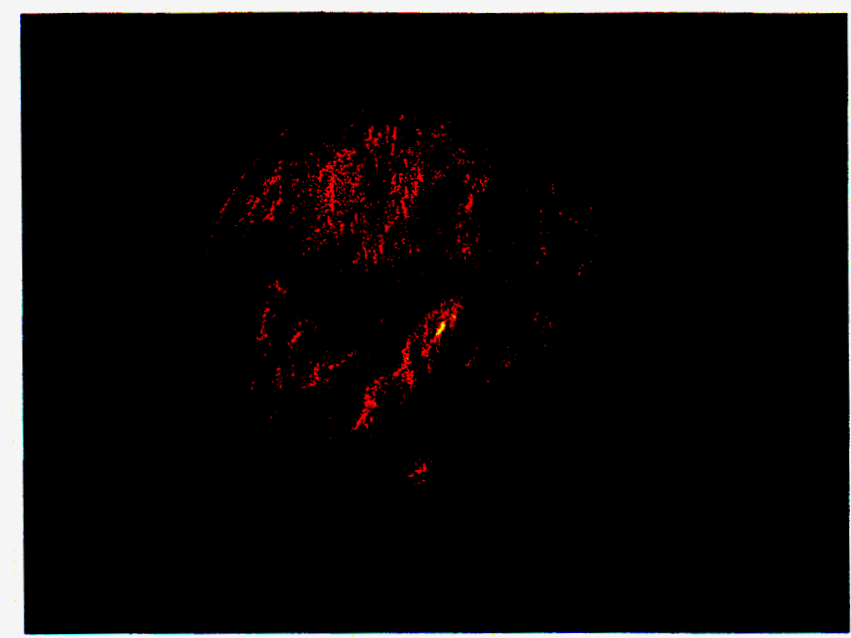

Figure 13a: Two Coatings, Both Soft-Baked, on Oxygen Etched CH (sample \#9)

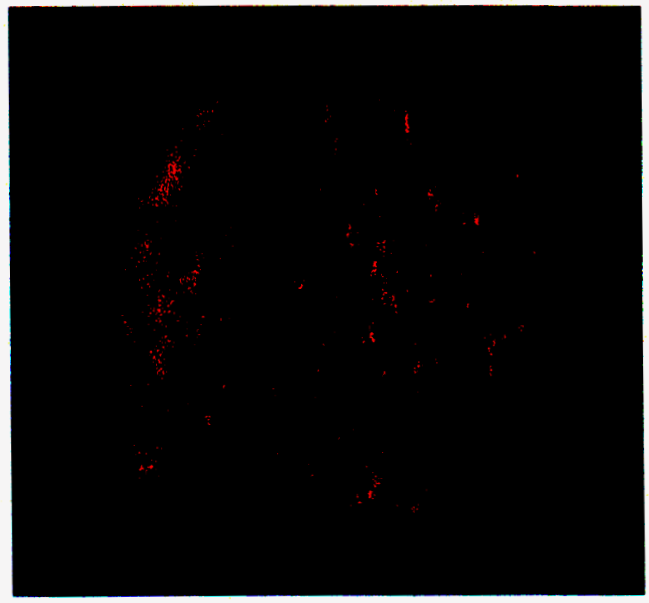

Figure 13b: $\sim 3 X$ zoom of Fig. 13a

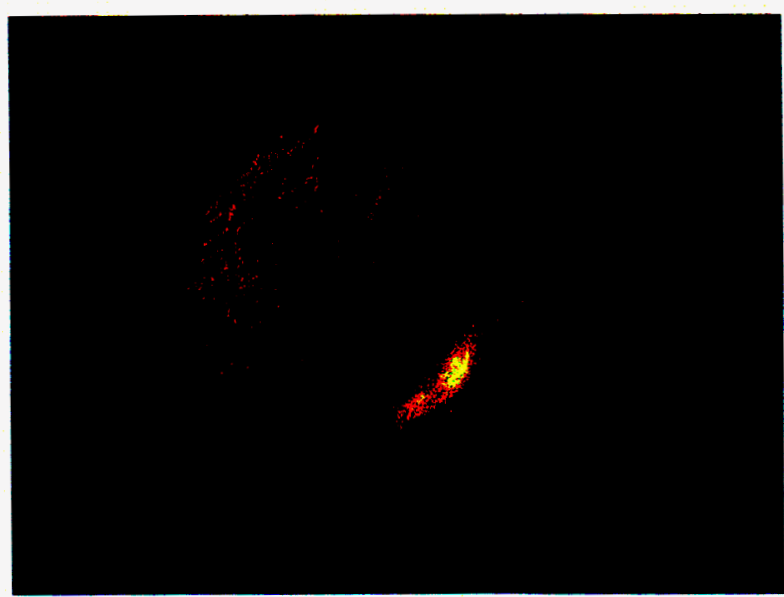

Figure 14a: Partly Coated, not Soft-Baked, 25\% PA w/0.5\% surfactant on untreated CH (sample \#10) 


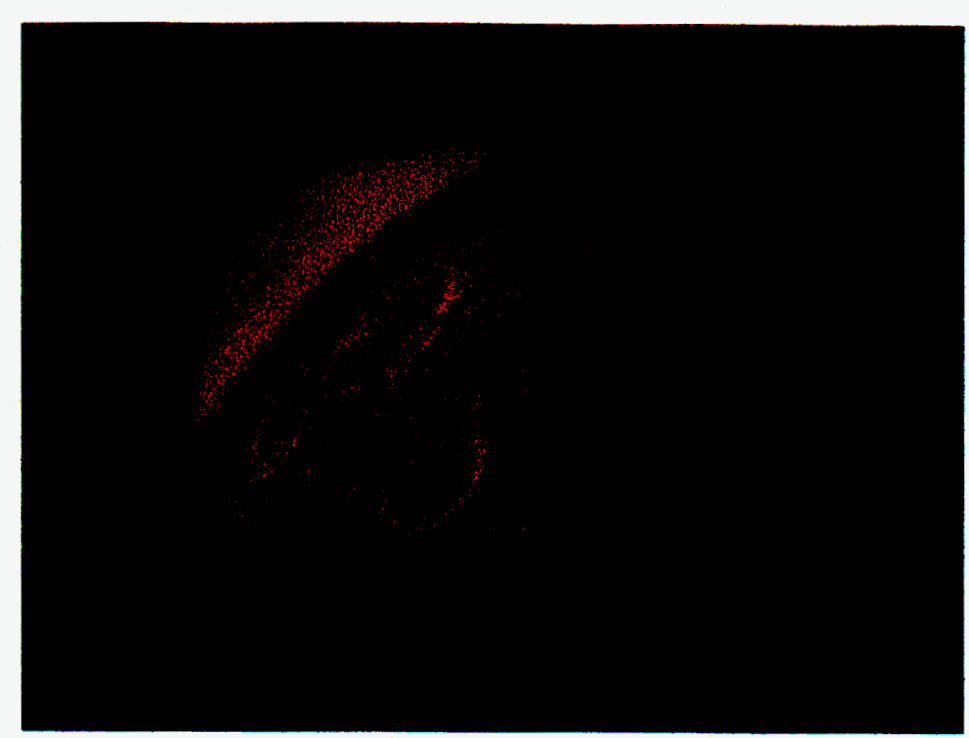

Figure 14b: $\sim 3 \times$ zoom of Fig. 14a

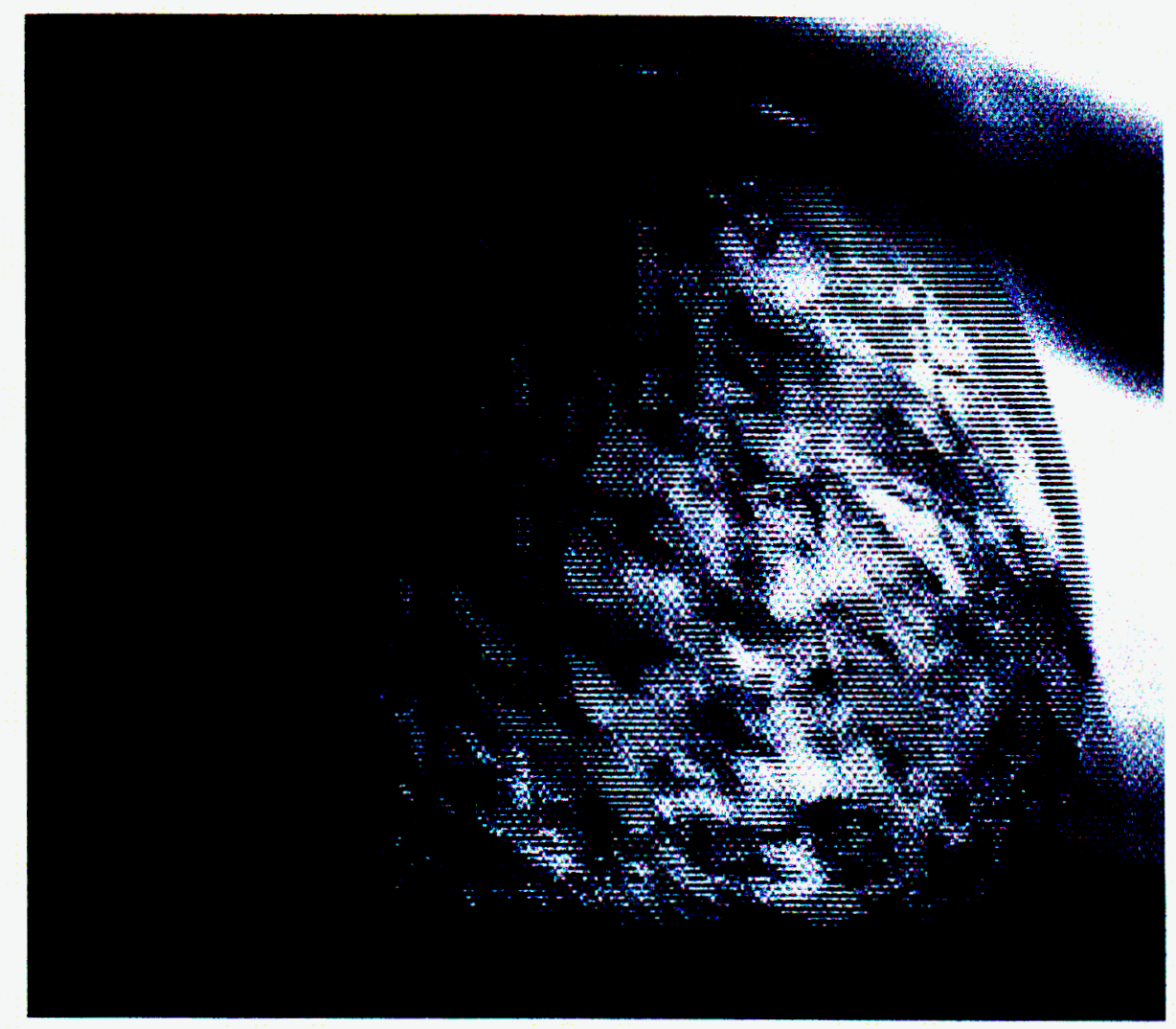

Figure 15: Back lit Mandrel (sample 8) 


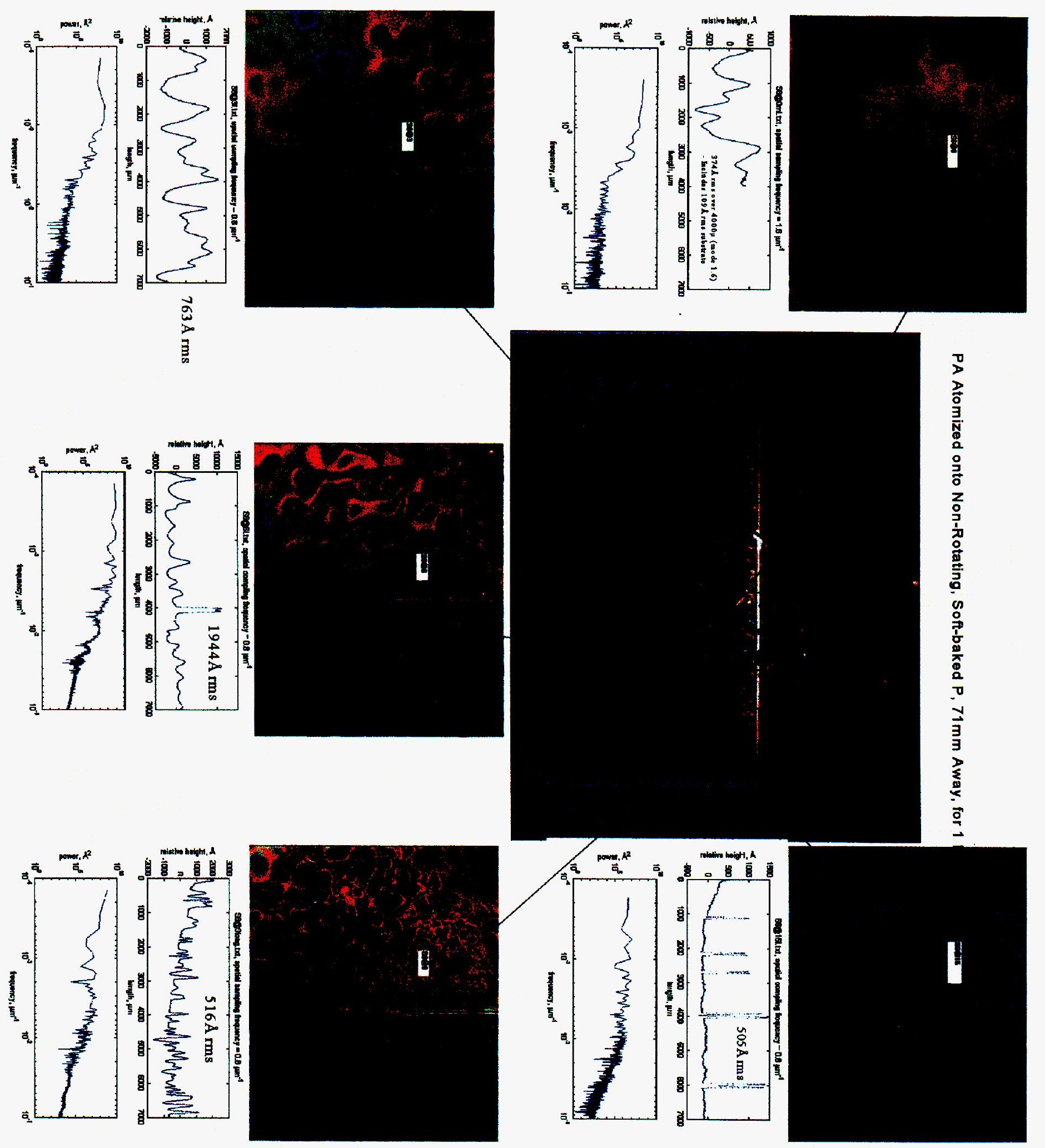

Figure 16 


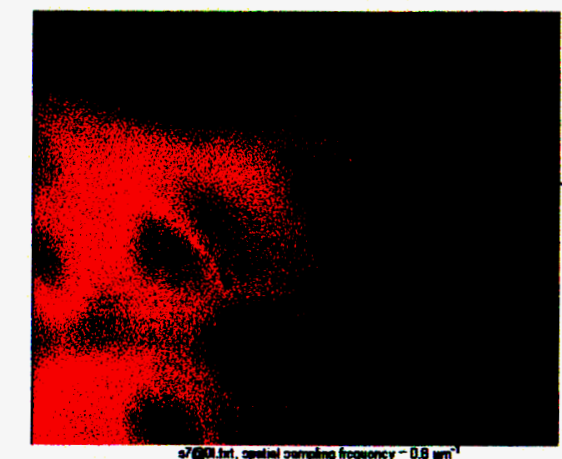

PA Atomized onto Soft-baked P, Rotating at 3RPM, $71 \mathrm{~mm}$ Away, for 1

1
8
8
0
0
0
0

$\vec{A}$
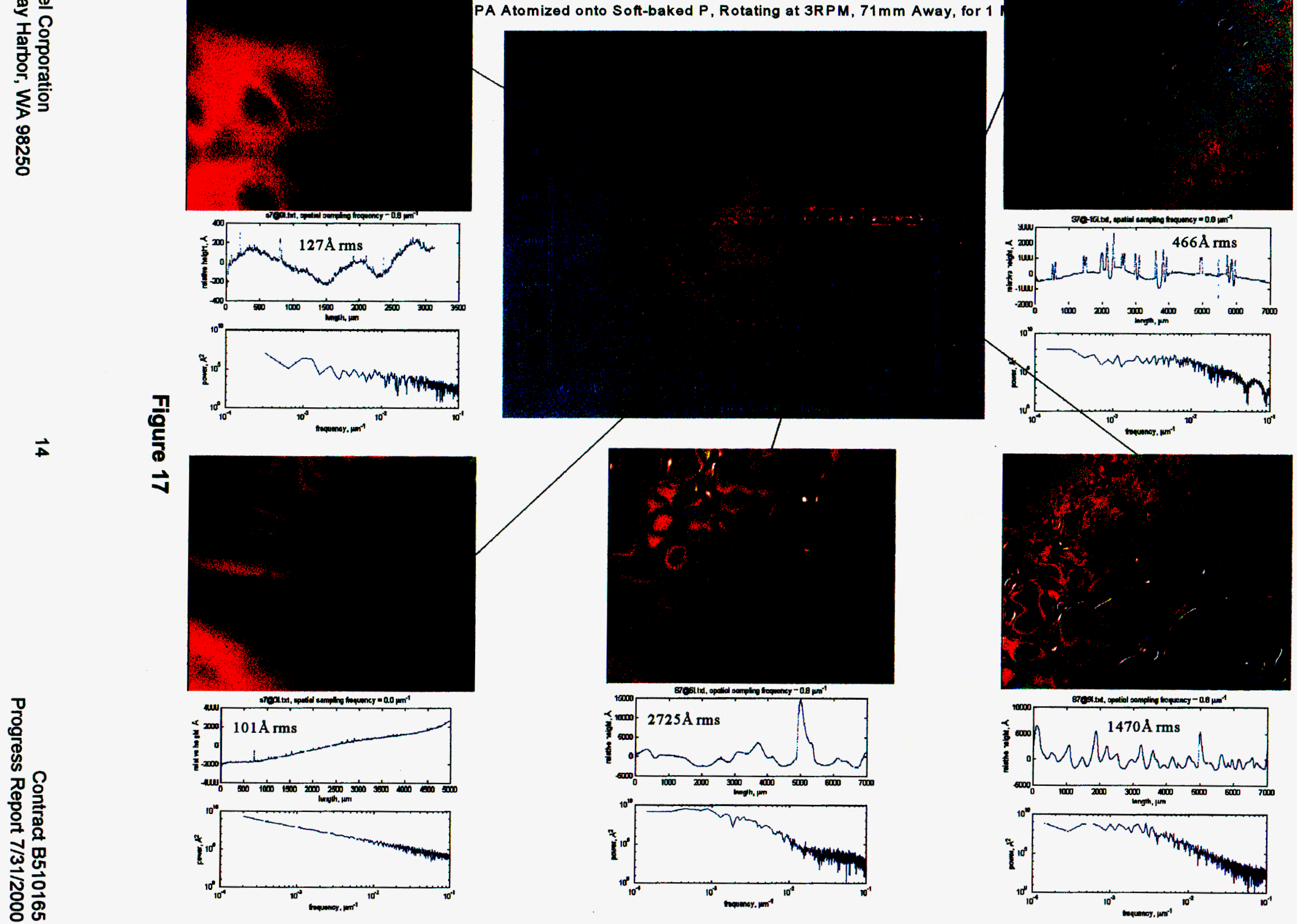


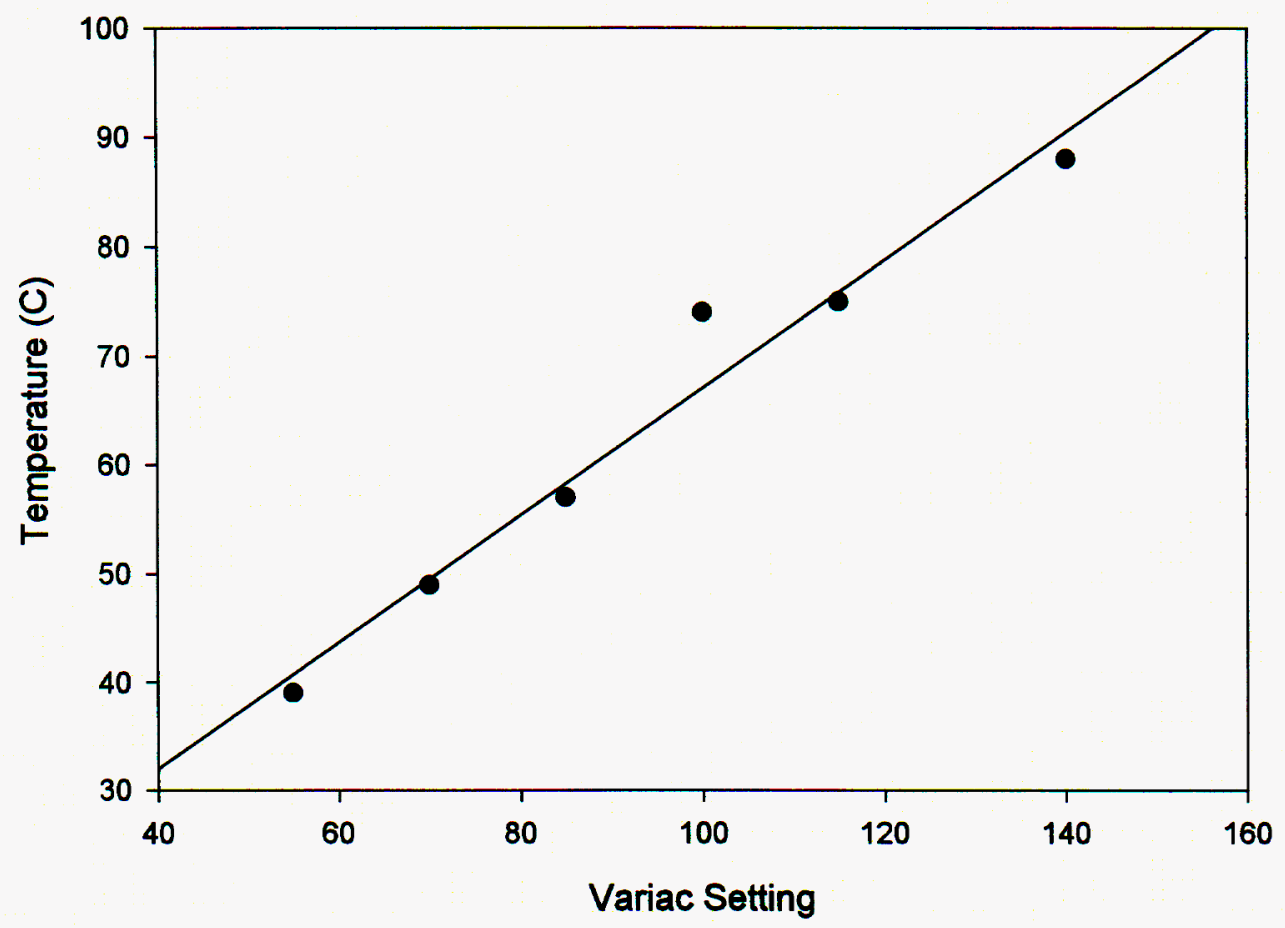

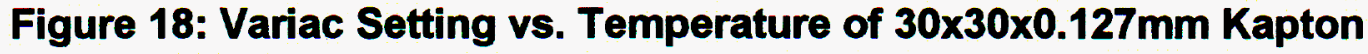
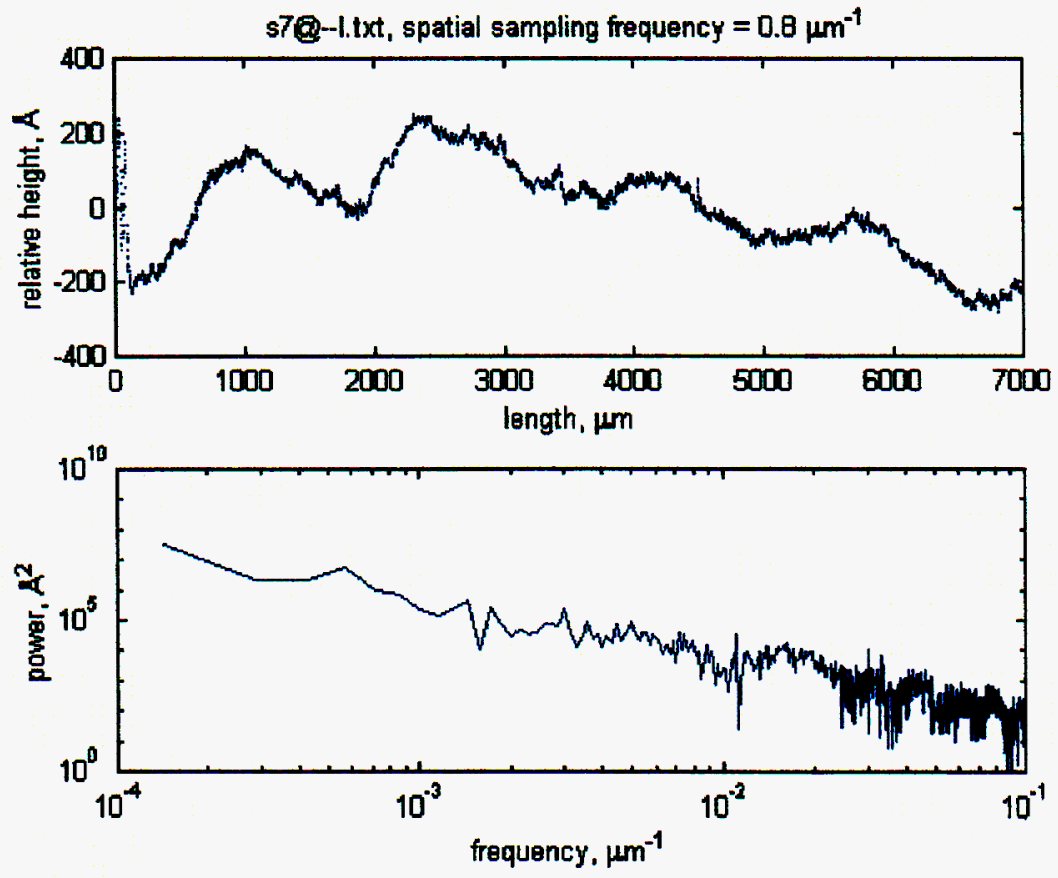

Figure 19: Surface Profile and 1-D Power Spectra of Substrate (Silicon with 1400A Spun-Cast P) 
Surface Roughness vs. Position

Figure 20 
Surface Roughness vs. Thickness

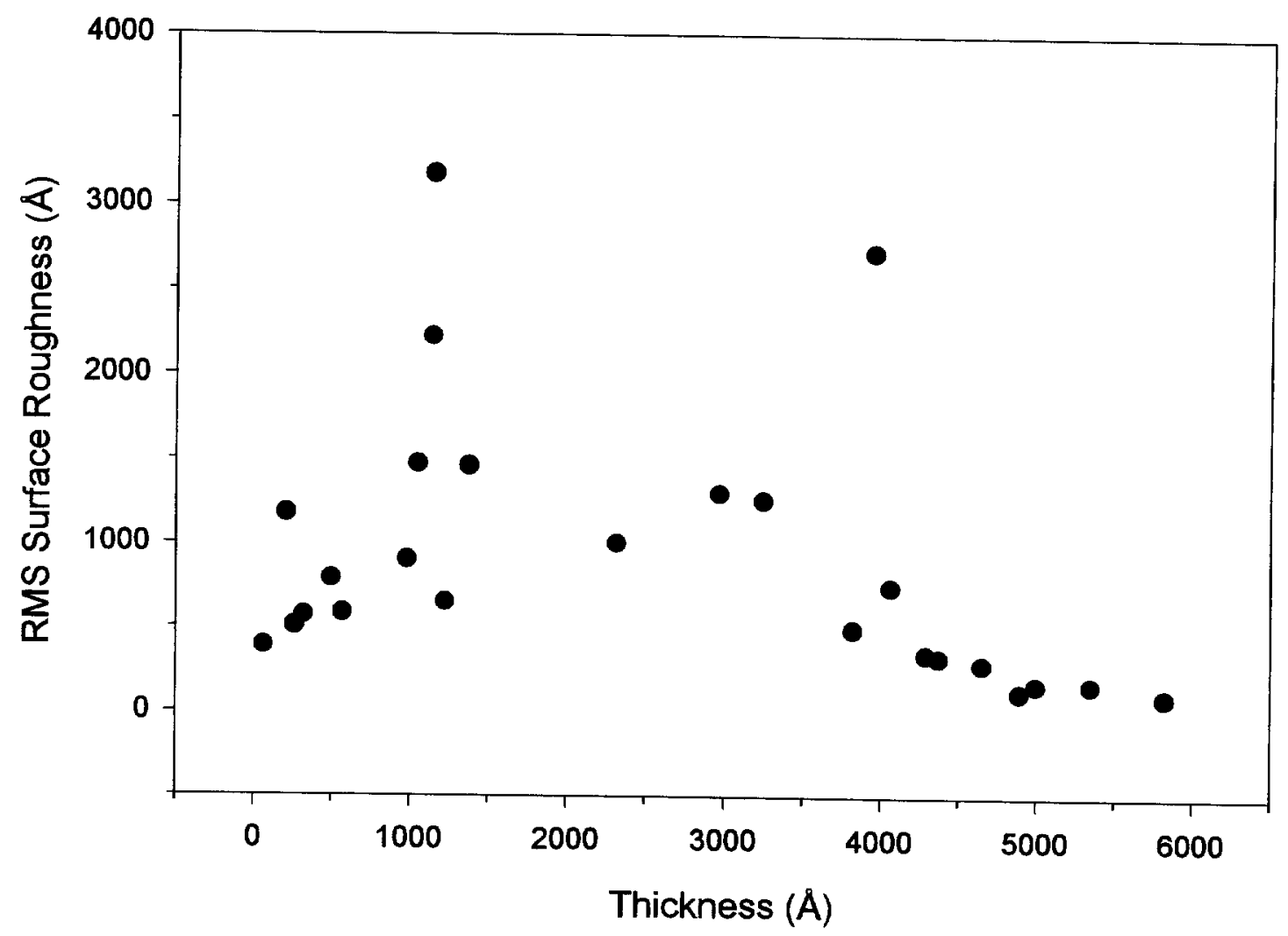

Figure 21 


\begin{tabular}{|c|c|c|c|c|c|c|c|c|c|}
\hline Sample & $\begin{array}{l}\text { PA flow } \\
\text { rate, } \mathrm{ml} / \mathrm{hr}\end{array}$ & $\begin{array}{l}\text { Air flow } \\
\text { rate, scfh }\end{array}$ & $\begin{array}{l}\text { Atomizer } \\
\text { power, } w\end{array}$ & $\begin{array}{c}\text { Depositio } \\
\text { n time, } \\
\text { min:sec }\end{array}$ & $\begin{array}{c}\text { Atomizer- } \\
\text { substrate } \\
\text { distance, } \\
\mathrm{mm}\end{array}$ & $\begin{array}{c}\Delta t \\
\text { between } \\
\text { end of } \\
\text { deposition } \\
\text { and start } \\
\text { of } \\
\text { heating, } \\
\text { sec }\end{array}$ & $\begin{array}{c}\text { Heat } \\
\text { distance, } \\
\mathrm{mm}\end{array}$ & $\begin{array}{c}\text { Heating } \\
\Delta t, \\
\text { min:sec }\end{array}$ & $\begin{array}{l}\text { Rotation } \\
\text { rate, RPM }\end{array}$ \\
\hline 14 & 0.05 & 1.8 & 2.5 & $1: 00$ & 45 & 12 & 20 & $5: 30$ & 3.9 \\
\hline 15 & 0.1 & 1.8 & 2.5 & $1: 00$ & 45 & 12 & 20 & $-5: 00$ & 3.9 \\
\hline 17 & 0.1 & 1.8 & 2.5 & $0: 40$ & 45 & 11 & 20 & $6: 00$ & 5.6 \\
\hline 18 & 0.1 & 1.8 & 2.5 & $0: 20$ & 45 & 11 & 20 & $5: 00$ & 6.4 \\
\hline 19 & 0.05 & 1.8 & 2.5 & $0: 16$ & 45 & 26 & 20 & $\sim 5: 00$ & 8.1 \\
\hline 20 & 0.05 & 1.0 & 2.5 & $0: 17$ & 45 & 11 & 25 & $\sim 5: 00$ & $\overline{14.7}$ \\
\hline 26 & 0.05 & 1.8 & 2.5 & $2: 00$ & 71 & 12 & 25 & $-5: 00$ & 29.6 \\
\hline 27 & 0.05 & 1.8 & 2.5 & $2: 20$ & 71 & 16 & 25 & $\sim 5: 00$ & 29.6 \\
\hline
\end{tabular}

Table 2 


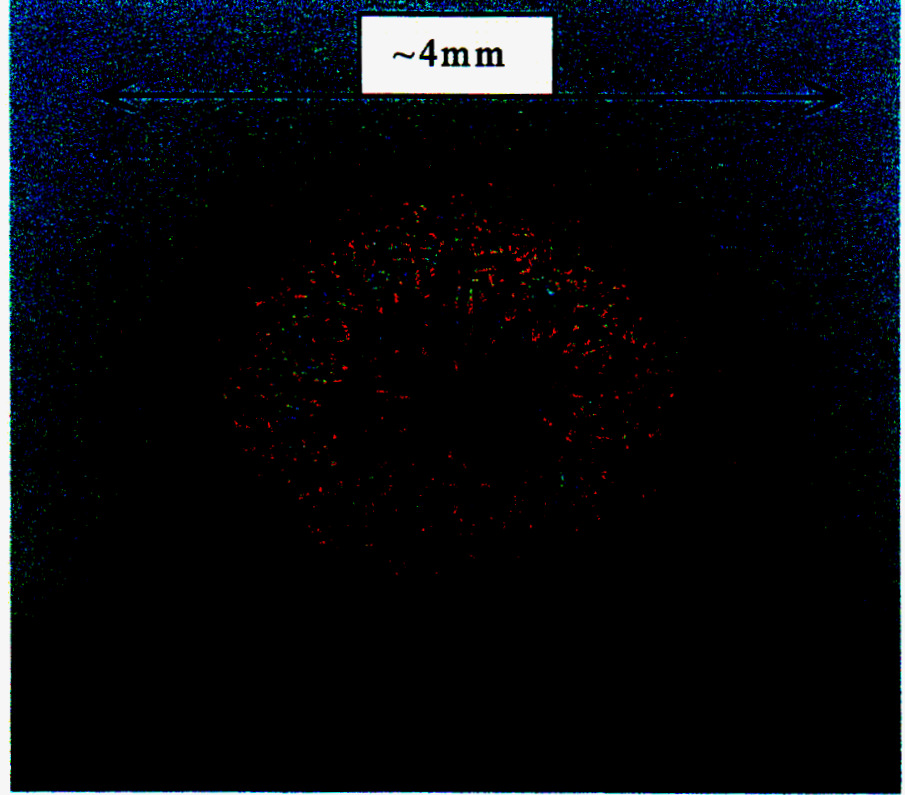

Figure 22: Before Replenishing PA Supply, Sample \#26

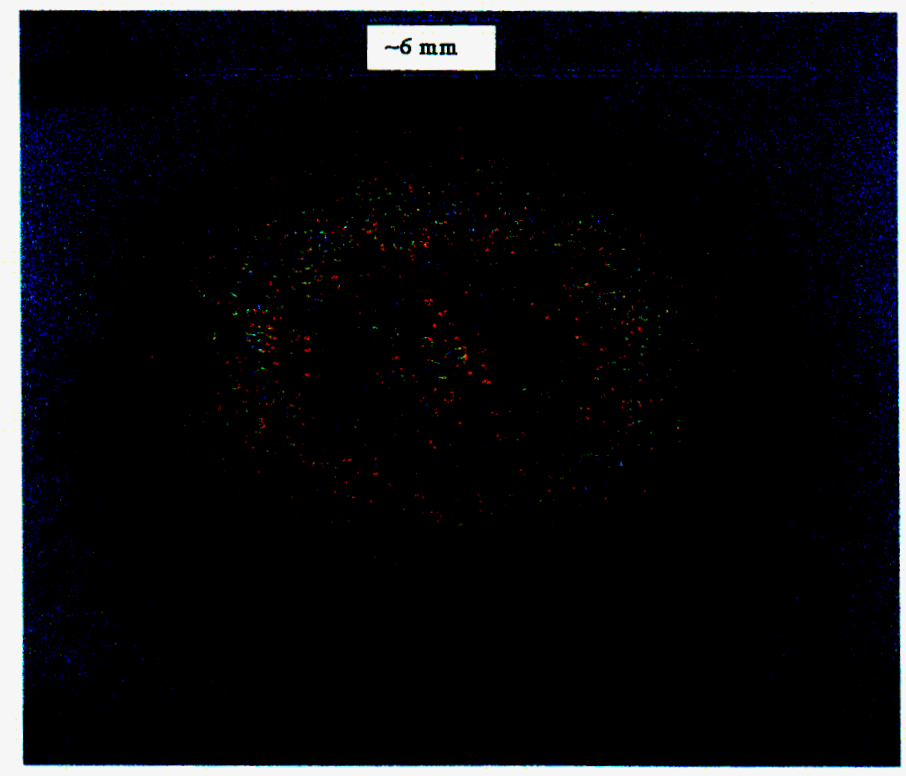

Figure 23: After Replenishing PA Supply, Sample \#27 


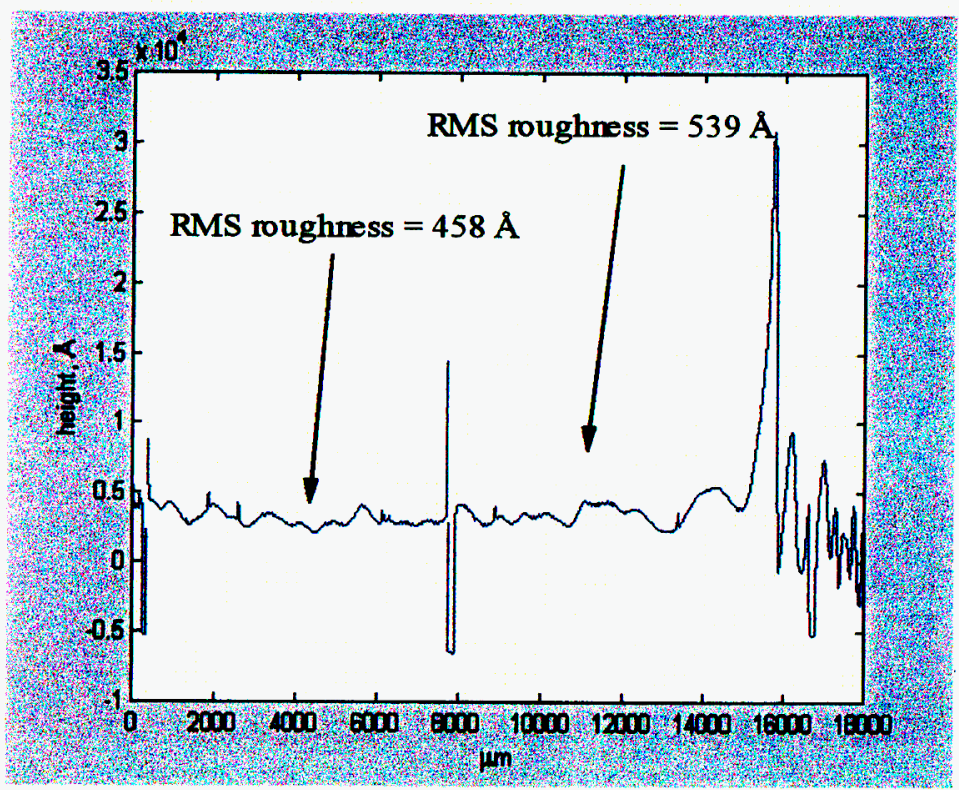

Sample 14
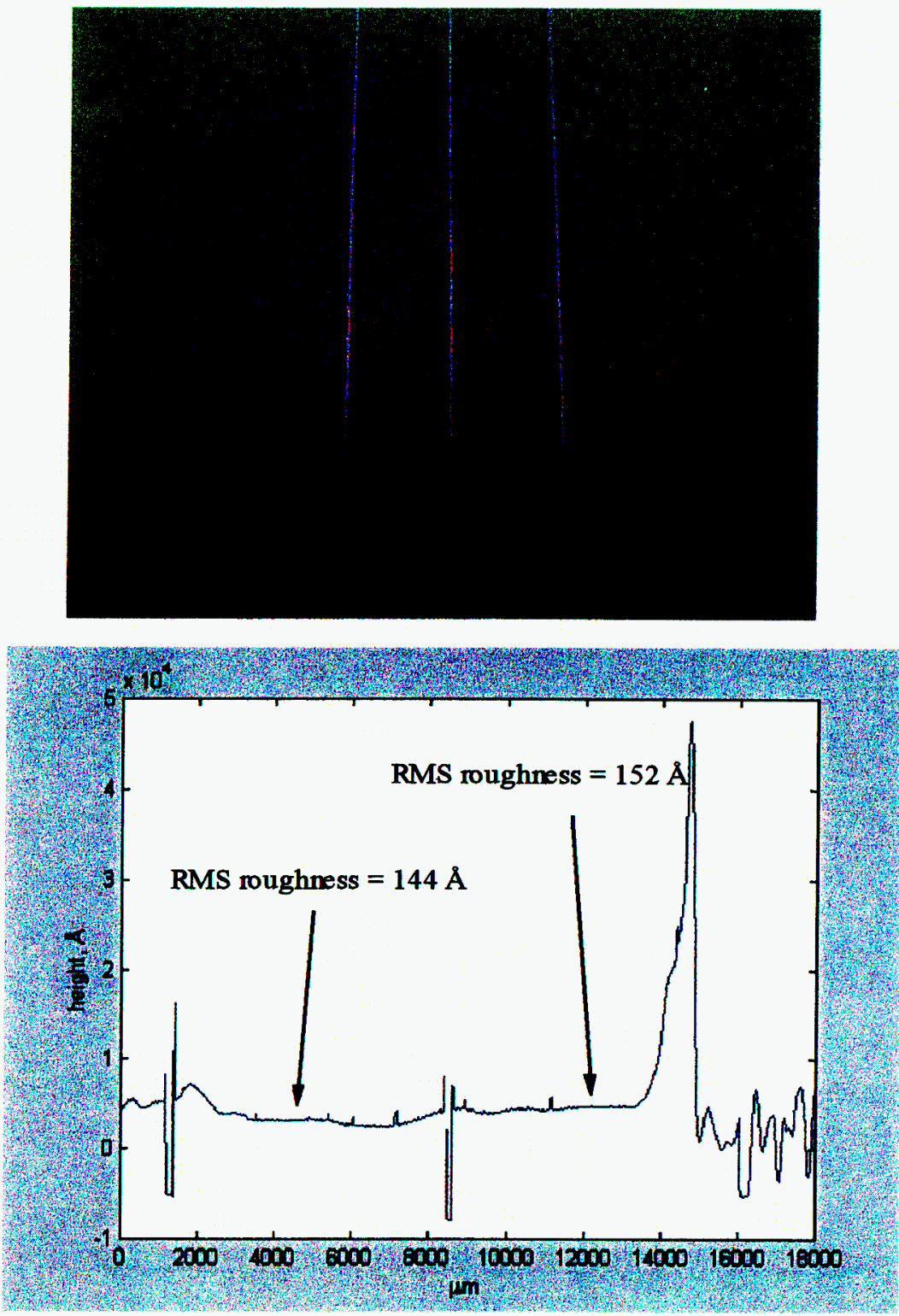

온

กิ

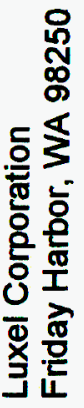




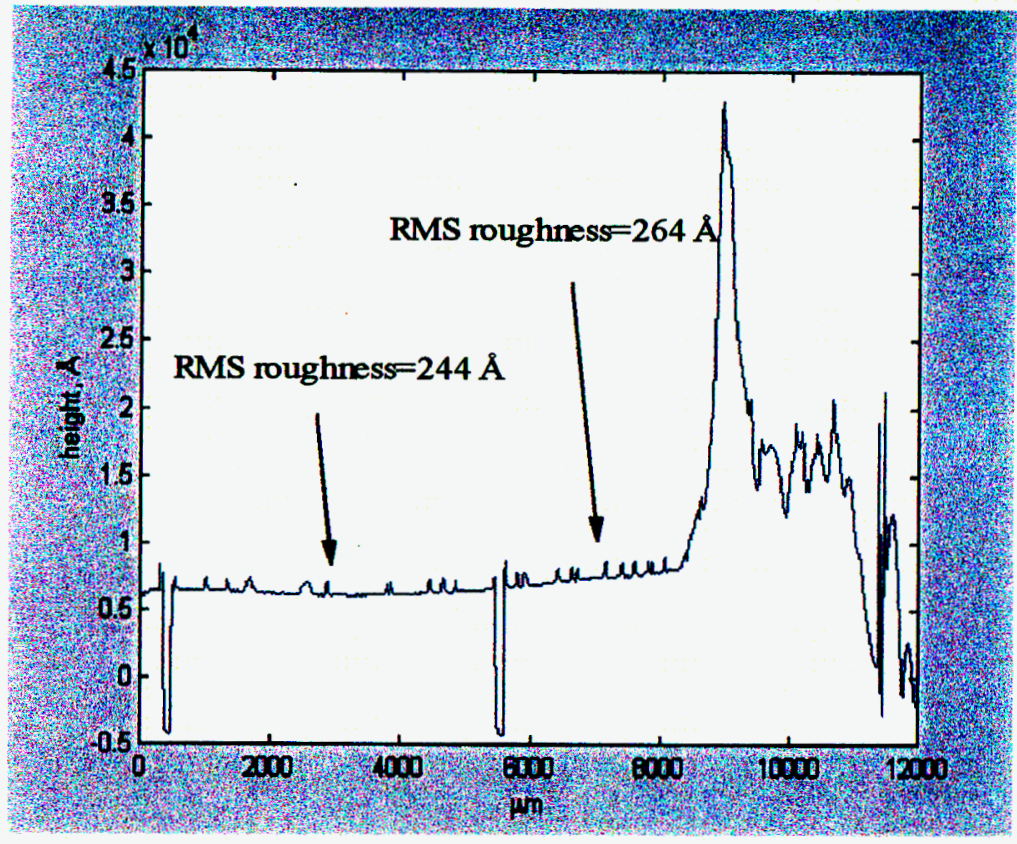

Sample 17
8

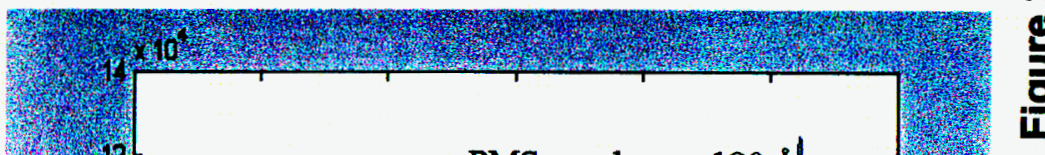

논

$\bar{N}$

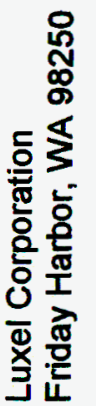



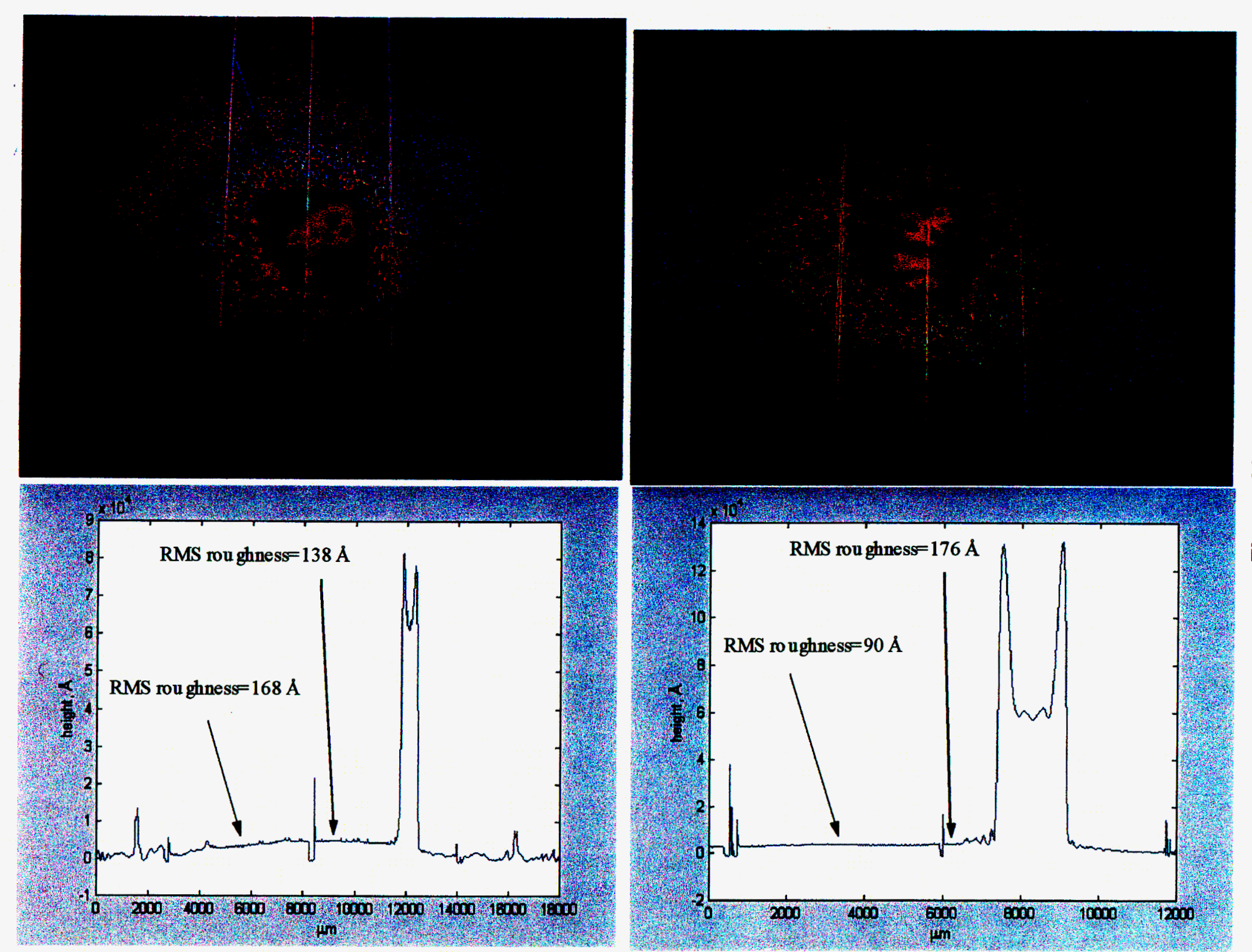

ㅇ
녹
은 


\section{Final Progress Report:}

\section{Investigation of Spray Conditions Necessary for Smooth Polyimide Microspheres}

The first progress report (5/15/2000) covering part one of the contract period investigated the spray conditions required to coat $\mathrm{CH}$-spheres with $\mathrm{P}$ (polyimide). The main conclusions of that report are:

1. Ultrasonic atomization is an appropriate means of dispensing PA (polyamic acid) if aggravated conditions of high atomization power ( 20 watts) and low PA flow rate are avoided.

2. PA dry spay is avoided if aggravated conditions of high air-flow rate through the atomizer and large radial distances $(>3 \mathrm{~cm})$ from the central atomized stream are avoided.

3. Dramatic improvement in the wettability of PA on $\mathrm{CH}$-sphere material is observed when using oxygen-etched $\mathrm{CH}$-spheres.

4. Manifestations of static buildup during atomized deposition of PA onto gas-levitated $\mathrm{CH}$-spheres have not been observed.

Details regarding these conclusions may be found in the first report.

The second progress report $(7 / 31 / 2000)$ covering part two of the contract period continued investigating spray conditions necessary for smooth $\mathrm{P}$ (Polyimide) coatings. Significant findings of that report are:

1. The solvent used in PA (Polyamic Acid), NMP, evaporates at low temperatures $\left(\sim 60^{\circ} \mathrm{C}\right)$ in a short amount of time $(\sim 1$ minute) without loss of wettability for subsequent PA on soft-baked P.

2. It is possible to apply a smooth-looking $\mathbf{P}$ film over a rough-looking one.

3. A smoothing transition for PA sprayed onto vertically mounted, rotating substrates occurs at a soft-baked $P$ thickness of $\sim 3500 \AA$ with RMS roughness transitioning from $\sim 500$ to $\sim 150 \AA$.

Details regarding these findings may be found in the second report.

During the third and final part of the contract we focused primarily on coating stalkmounted $\mathrm{CH}$-spheres with $\mathrm{P}$, and secondarily on coating acoustically levitated $\mathrm{CH}$ - 
spheres with our recently acquired polymer ink-jet dispenser system. The following is discussion of the findings and progress made during this final contract period.

Significant progress has been made in coating stalk-mounted $\mathrm{CH}$-spheres with $\mathrm{P}$ via atomization. We have developed an automated system for coating and in-situ softbaking. This system coats at a rate of $\sim 1.5 \mu \mathrm{m}$ of soft-baked P per hour. We have also successfully imidized coated $\mathrm{CH}$-spheres resulting from this automated system. We have continued developing an in-situ shadowgraphy tool for quantifying the distribution surface roughness on coated CH-spheres. This tool calculates PSD's (power spectral density) of plane-projected, edge-detected closed curves of coated $\mathrm{CH}$-spheres. Current results indicate that mode 2 non-uniformity is $\sim 12 \mu \mathrm{m}$. Radiography measurements performed at LLNL indicate that multi-layer, soft-baked polyimide coatings form a singular, homogeneous coat about $\mathrm{CH}$-spheres.

Our automated system for coating and soft-baking is shown in figure 1. It consists of an axially rotating, stalk-mounted (with high temperature epoxy) $\mathrm{CH}$-sphere surrounded by a heating coil for soft-baking. A pneumatic-controlled shutter covers/uncovers a continuously operated atomizer source of PA that is aimed at a rotating $\mathrm{CH}$-sphere, transverse to the axis of rotation. The atomizer is fed PA via a syringe pump. A thermocouple temperature sensor is located in the center of the heating coil and is $\sim 3$ $\mathrm{mm}$ from the $\mathrm{CH}$-sphere. This is used to measure approximate air temperature surrounding the $\mathrm{CH}$-sphere. The heating coil, temperature sensor, and pneumaticcontrolled shutter are connected to a LUXEL Radak power controller unit which runs a closed-loop program to open the shutter for a set time (depositing PA onto a rotating $\mathrm{CH}$ sphere), close the shutter, and then initiate a soft-bake cure cycle. The currently used program is depicted in figure 2 and may be repeated indefinitely.

Problems attributable to improper soft-baking have apparently been solved using our current automated system. These problems (using the previous heat-lamp setup) were: collapsing of $\mathrm{CH}$-spheres, condensation on the inside surface of $\mathrm{CH}$-spheres, and blistering. We also did not have good calibration of the approximate temperature surrounding a coated $\mathrm{CH}$-sphere using the heat lamp, which was too dependent on distance from the sample. If too much heat was applied early in the soft-bake cycle, samples collapsed, probably due to the formation of a hard shell on the outside surface that prevented solvent from diffusing out. This problem seems to have been solved by heating more slowly, initially. Condensation on the inside surface of a $\mathrm{CH}$-sphere occurred after an extended (12 hours) soft-bake time where, apparently, hot solvent had time to diffuse into the interior of the sample and condensed when a new coating was applied which suddenly cooled the sample. This problem is now apparently avoided by incorporating a cool-down period after extended heating. It is interesting to note that we did not see this problem occurring after shorter ( $45 \mathrm{~min}$ ) soft-bake times even though coated $\mathrm{CH}$-spheres were heated to a maximum at the end of the soft-bake cycle and recoated immediately after the end of the cycle. It seems that diffusion takes some time to 
occur. The third issue, blistering, occurs as a result of insufficient soft-baking where solvent from previous coatings is trapped beneath subsequent coatings. Blistering began to occur, getting progressively worse, after roughly the sixth coating. A longer, hotter cycle is being used now, with radiant heat, which seems to avoid the blistering problem.

Using our automated coating and soft-baking system, we have successfully coated two $\mathrm{CH}$-spheres with $\sim 25$ and $\sim 70 \mu \mathrm{m}$ of $\mathrm{P}$-the former is shown in figure 3 . These quantities where measured by Dr. Cook's group at LLNL using radiography. The edgedetected, plane-projection of the $\mathrm{CH}$-sphere in figure 3 is shown in figure 4 along with a fit of an ellipse to the edge; 1 pixel $\sim 6 \mu \mathrm{m}$. Note that the ratio of the major to minor axes, $a$ and $b$, is 1.01 , indicating a significant absence of macroscopic slumping. Figure 5 plots $\Delta$ radius versus $\theta$ of the edge-detected data and the ellipse fit to this data. This figure indicates that for this 1-D equatorial scan, our measuring system indicates the coated $\mathrm{CH}$-sphere is at most $\sim 12 \mu \mathrm{m}$ non-uniform at mode 2 . Figure 6 is the PSD of the data curve in figure 5-the 1-D Haan NIF specification is shown for comparison.

Both coated $\mathrm{CH}$-spheres have been successfully imidized, without falling off their stalks. The high temperature cure cycle occurs in an argon atmosphere starting at $\sim 200^{\circ} \mathrm{C}$. This temperature is raised to $\sim 300^{\circ} \mathrm{C}$ over 3 hours, then dwells for 30 minutes, and finally is lowered to $\sim 150^{\circ} \mathrm{C}$ over 3 hours. 


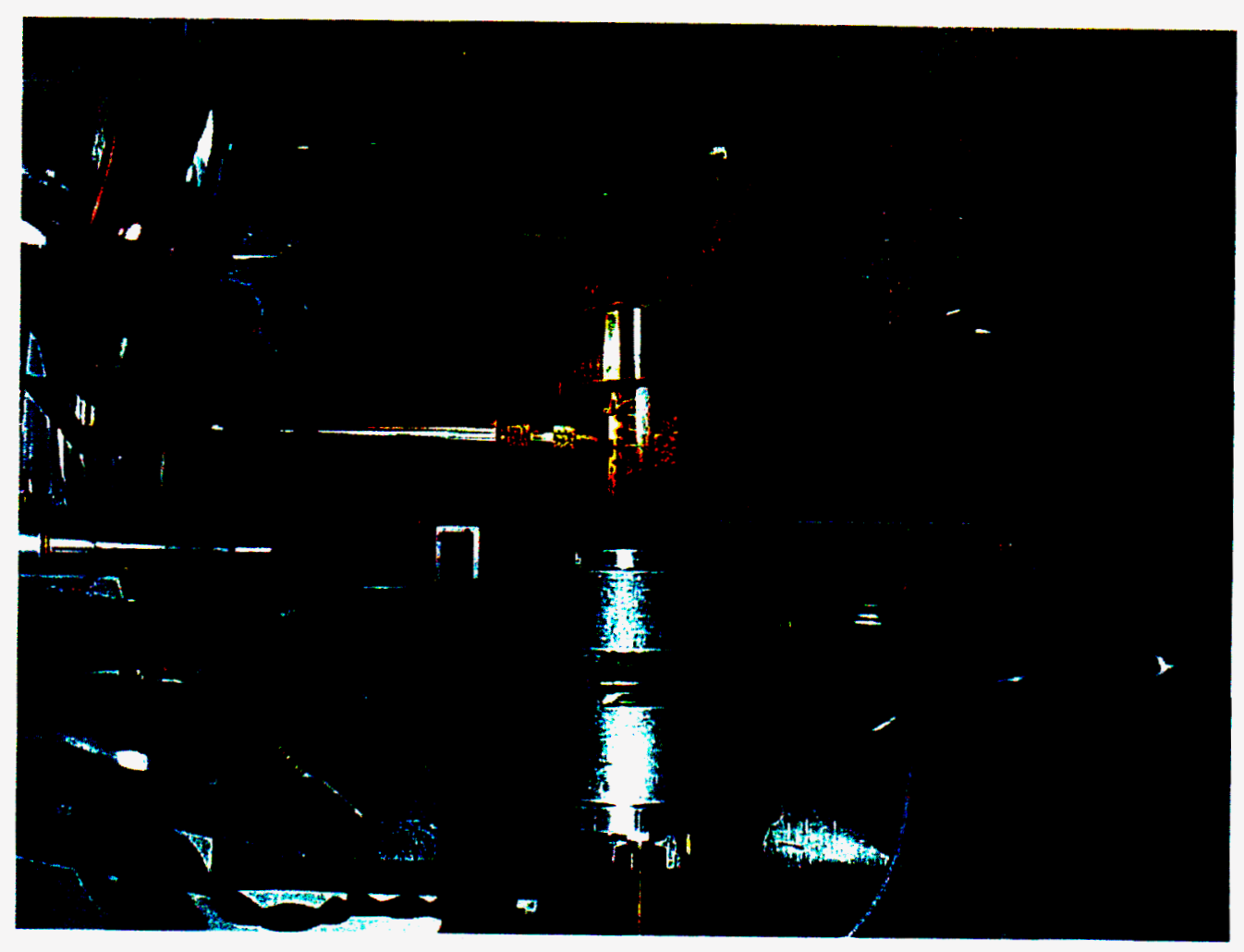

Figure 1

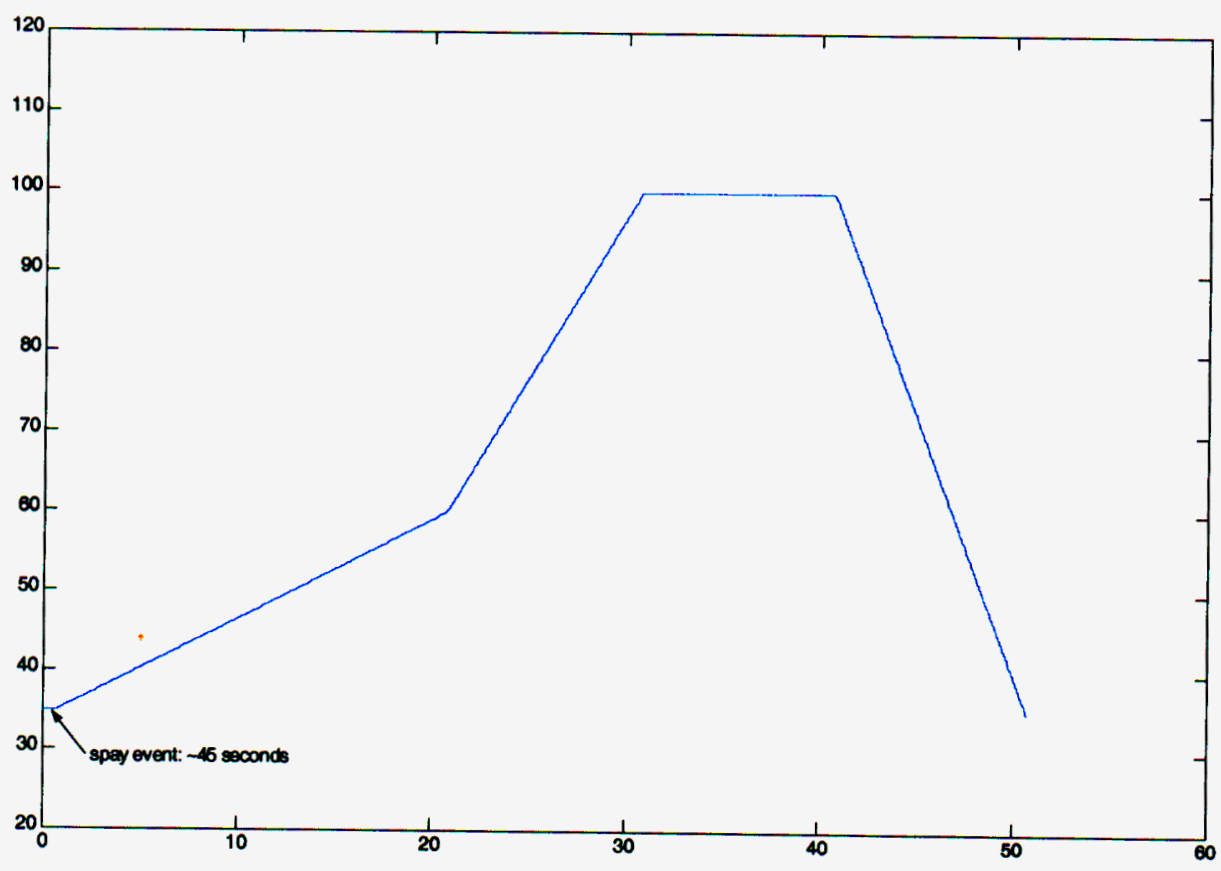

Figure 2

Luxel Corporation

Friday Harbor, WA 9825 


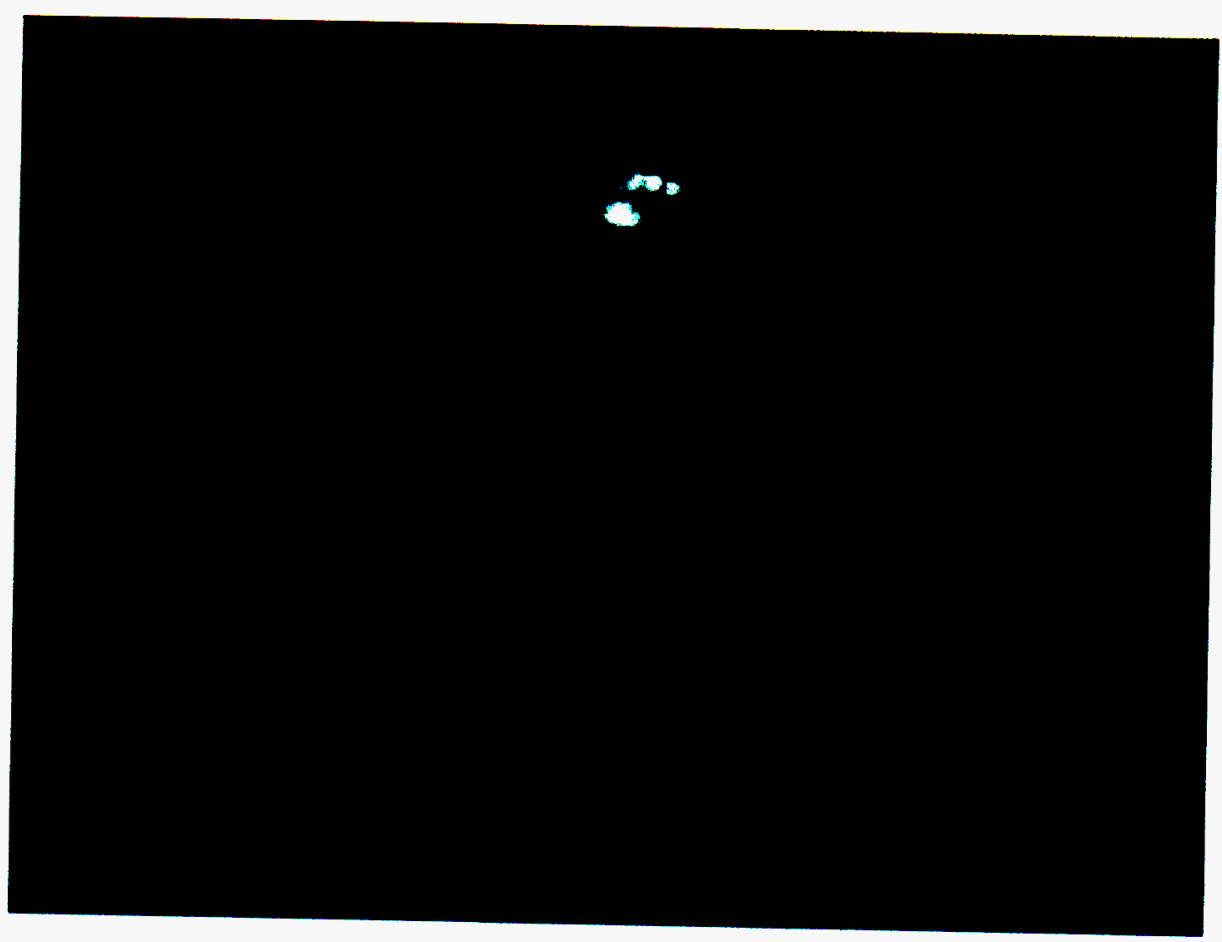

Figure 3

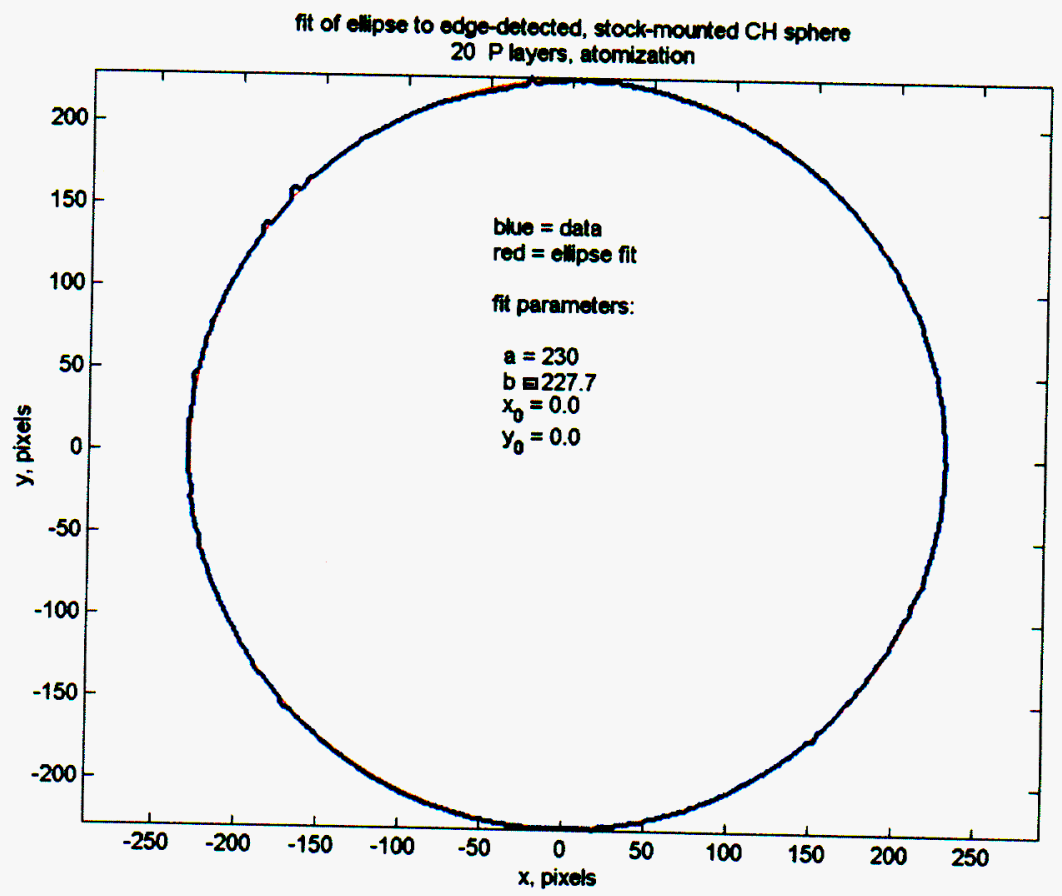

Figure 4

Luxel Corporation

Friday Harbor, WA 9825 


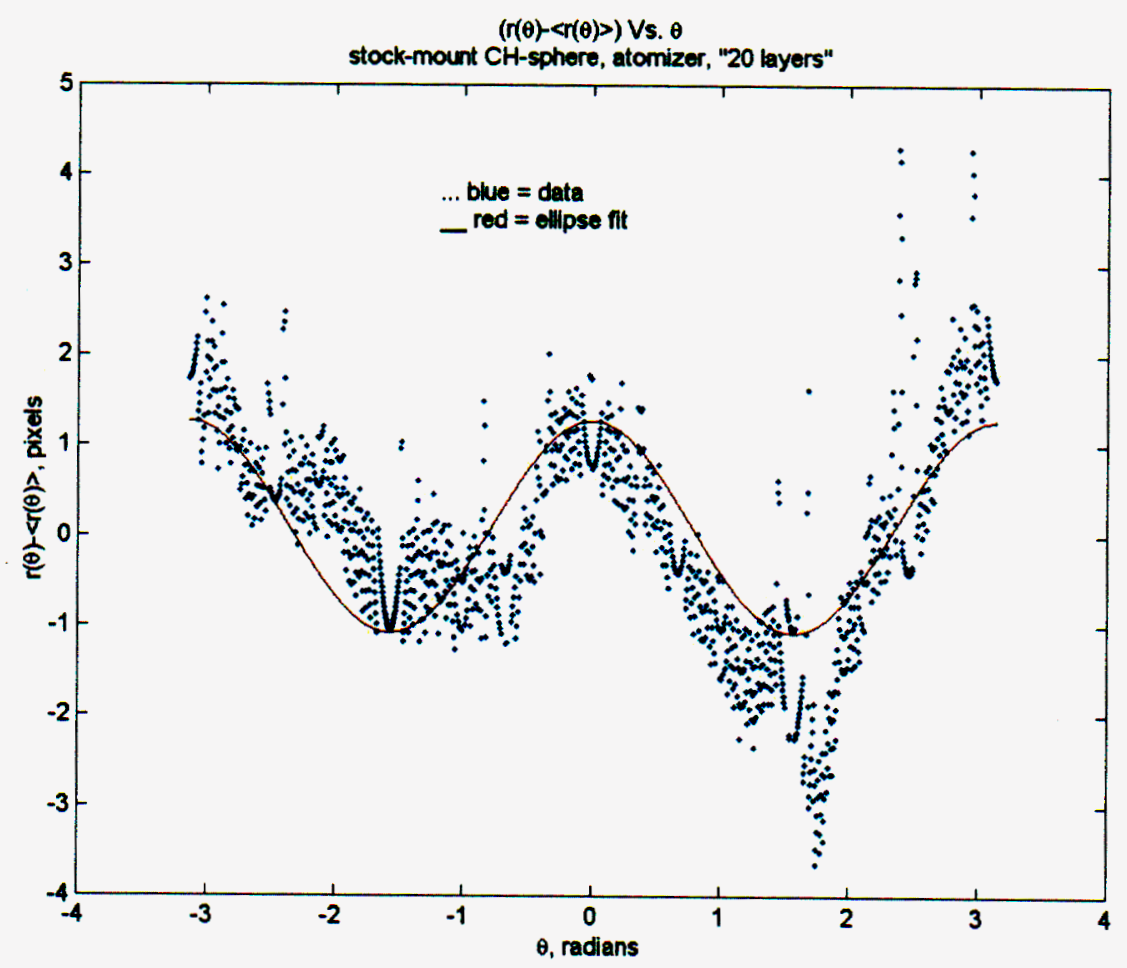

Figure 5

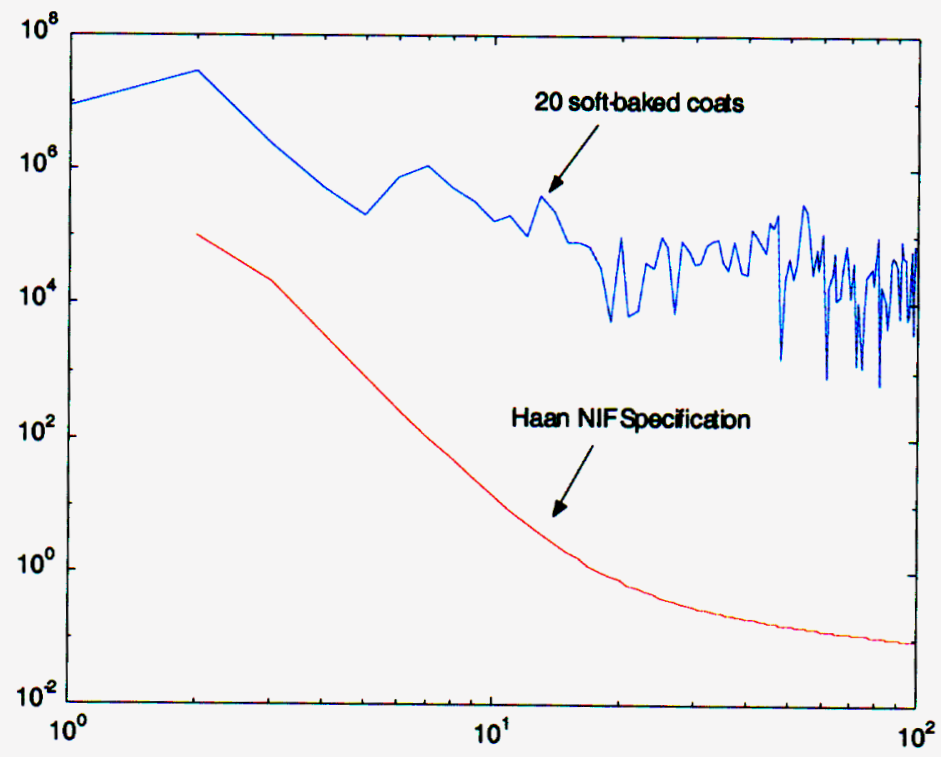

Figure 6 


\section{First Progress Report:}

\section{NIF Polyimide Ablators Produced by Solution Spray Techniques}

This first work period was occupied designing and building a clean-room workspace and investigating the availability of suitable experimental equipment.

The clean room workspace consists of a flow through table situated beneath downward flowing HEPA filtered air. The table has been designed to double as an optical bench for holding $1 / 2$ " vertical mounting rods to allow adaptable and secure locating of equipment. The bench area can be sealed off to provide undisturbed air with the HEPA unit turned off, for non-turbulent deposition conditions. After deposition, an exhaust louver is opened and the HEPA unit turned on to push fumes outside without creating low pressure in the work area that would draw in contaminants from the room. This arrangement should provide a class- 100 clean area. The room outside the bench area is itself supplied with HEPA filtered air. The room can be partitioned with a vinyl soft-wall to provide $30 \%$ coverage and near class -1000 cleanliness. Walls are finished with high gloss paint that is less than ideal but adequate for class-1000. Three of the four 2' $x$ 4' HEPA units in the ceiling recirculate air and the other provides positive pressure in the room. The construction of the clean room is not being charged to the contract.

We are considering the experimental equipment which will be necessary to produce $>50 \mu \mathrm{m}$ thick coatings with thickness non-uniformities less than $1 \mu \mathrm{m}$, over a length of $100 \mu \mathrm{m}$ at the equator of stalk mounted mandrels by April 20th, 2001. Radiant heating as well as convective soft-bake heating will be used. An infrared lamp that uses an eliptical reflector to focus light onto a 1/4" spot has been found (item 1. on the Equipment List). The lamp can be incorporated into a closed loop control system with a thermocouple or pyrometer similar to the system used under the previous contract with convective heating. A maximum heat flux density of 150 Watts $/ \mathrm{cm}^{2}$ can be produced, which may be intense enough to produce imidization temperatures at the mandrel. The radiant energy at maximum output is centered at a temperature of $2500^{\circ} \mathrm{K}$, in the near-infrared at $1.2 \mu \mathrm{m}$ wavelength, but moves toward the mid-infrared at lower power, where we will most likely be operating. There are lamps that operate more exclusively in the mid-infrared where polyimide absorbs more readily, but their intensity is still less in the mid-infrared than the lamp centered at $2500^{\circ} \mathrm{K}$. Another advantage of soft-baking with near infrared energy is penetration of heat into the interior of the coating to help avoid a skinning over effect.

Non-contact temperature measurement of polyimide films thinner than $50 \mu \mathrm{m}$ requires a pyrometer optimized for a polyimide absorption band since polyimide is transparent over much of the infrared spectrum. The pyrometer must also be able to image a $2 \mathrm{~mm}$ target. A pyrometer that operates in a narrow region

Luxel Corporation

Friday Harbor, WA 98250
Task Order No. B514571

Master Task Agreement No. MTA B514570

Progress Report 12/22/2000 
centered at $3.43 \mu \mathrm{m}$ with a close focus lens to image a $3.2 \mathrm{~mm}$ diameter target has been found (item 2. on the Equipment List). We are expecting a demonstration unit January $8^{\text {th }}$. To find the temperature of a target smaller than the detector field of view, the background temperature must be taken into account. The signal produced by the pyrometer represents the average temperature within its field of view. Accordingly, the signal can be represented by the following equation where $T$ is the output signal, $T_{T}$ the target temperature, $A_{T}$ the target area, $T_{B}$ the background temperature, $A_{B}$ the background area as seen by the pyrometer, and $A$ the total area seen by the pyrometer:

$$
T=\left(T_{T} A_{T} / A\right)+\left(T_{B} A_{B} / A\right)
$$

The reduction in signal due to a target smaller than the detector field of view is mathematically identical to a reduction in emissivity and can be calibrated out the same way. The pyrometer we have specified has an accuracy of $\pm 1.5^{\circ} \mathrm{K}$. With a $2 \mathrm{~mm}$ diameter target at $50^{\circ} \mathrm{C}$ (a typical soft-bake-cycle temperature), background temperature of $20^{\circ} \mathrm{C}$, and a $3.2 \mathrm{~mm}$ field of view, accuracy decreases to $\pm 3.8^{\circ} \mathrm{C}$ (from the ratio of $A_{T}$ to $A$ ), which is adequate for our needs.

During this phase of the contract we will attempt to improve the deposition process by using an inkjet device. We will also attempt to broaden the area of uniform coverage to include more than just the equatorial region of the mandrel. Thus, for inkjet deposition onto stalk mounted mandrels, the print head will be mounted on a single axis micro-positioner to move the jet along the axis of rotation of the mandrel in order to provide more uniform coating coverage on the rotating mandrel. At first, the ink jet will be moved only along the equatorial region of the mandrel as it rotates in order to determine the droplet density necessary to produce a smooth coating and to duplicate the atomizer produced coatings. More complete coverage of the mandrel can be obtained either by varying the translational velocity of the inkjet while maintaining constant droplet frequency or by varying the droplet frequency while maintaining constant translational velocity. A third option would be to vary the mandrel's speed of rotation while keeping both translational velocity and droplet frequency constant but this scenario is not likely to be applicable to levitating mandrels. In the first case, the density of deposited droplets would be kept constant by varying the translational velocity - more slowly at the equator than at the poles. In this method, droplet density would be only nominally constant since the greater circumference at the equator would be covered by multiple rotations of the mandrel - where droplets would only occasionally be equally spaced. The second method, where droplet frequency varies while translational velocity is kept constant, would be preferable because droplet spacing could be kept constant across the mandrel. In this case, the droplet frequency would vary from 0 at the ends of the mandrel $(x=-1,1)$ to $\beta$ at the equator according to:

$$
f(x)=\beta \sqrt{1-x^{2}}
$$

Luxel Corporation

Friday Harbor, WA 98250
Task Order No. B514571

Master Task Agreement No. MTA B514570

Progress Report 12/22/2000 
This follows from the fact that the circumference of the circle created by the intersection of a plane perpendicular to the $x$-axis with a hollow sphere (the path of the polyimide droplets around the mandrel as it rotates) varies with $x$ according to the circle's radius, which is described by the equation of a circle. The constant, $\beta$, couples the inkjet's droplet frequency to the mandrel rotation such that the inkjet moves a distance in one mandrel-rotation that is equal to the distance between successive deposited drops. Given 50 $\mathrm{mm}$ diameter drops that spread out considerably on impact, a good starting point for this distance is $100 \mu \mathrm{m}$, or $62.8 \mathrm{drops} /$ rotation at the equator. At a typical mandrel rotation of 1 revolution-per-second, this is a droplet frequency of $62.8 \mathrm{~Hz}$. The ink-jet would translate at a constant velocity of $100 \mu \mathrm{m} / \mathrm{s}$. The exact quantities to provide uniform smooth coatings will be the object of experimental effort. We do not know yet how difficult it will be to program droplet frequency. An appropriate stage will have to move smoothly and repeatably with at least $1 \mu \mathrm{m}$ resolution, preferably much better. The controller will have to allow programming and ideally also control mandrel rotation accurately. An appropriate stage, motor for rotating stalk-mounted mandrels, and a controller have been found (items 3,4 and 5 on the Equipment List).

In this manner, uniform coating thickness should be achieved over an area larger than just the equatorial belt. If this technique is successful, it may have application for a levitating mandrel depending on how well the rotation of the mandrel can be controlled. In practice, droplet frequency or translation will no doubt need to be modified in order to account for irregularities such as droplets impacting the mandrel at an angle. The polyimide droplet jet may also move around somewhat due to the distance away from the levitating target and deflection by the acoustic field, but on average, this technique should provide uniform droplet coverage. Surface tension should also help smooth out local variations in thickness.

A high-resolution digital camera has been purchased as well as a zoom lens for imaging mandrels. With edge detection we can theoretically obtain a resolution of $2 \mu \mathrm{m}$. This tool will be used to determine uniformity of coatings before softbaking as well as the final product. An advantage of the solution-based method is that typically, $97 \%$ of the mass and a corresponding volume is lost upon softbaking so that pre-soft-bake non-uniformities should shrink to something considerably less in the final coating.

Next, we plan to finish the clean room and clean bench area, make final decisions on equipment, submit the list for contractual approval, assemble the equipment, and begin depositions. The proposed additional equipment is listed on Attachment A. The financial performance under the contract to date is given on Attachment B.

Luxel Corporation

Friday Harbor, WA 98250
Master Task Agreement No. MTA B514570

Progress Report 12/22/2000 
Second Progress Report:

\section{NIF Polyimide Ablators Produced by Solution Spray Techniques}

While our class 100/1000 clean-room has been under construction, we have focused on establishing a polyamic acid (PA) ink-jet delivery system for coating stock-mounted $\mathrm{CH}$-shells. We are currently able to dispense on-demand, single $\sim 50$ micrometer diameter PA ( $3 \%$ by weight) droplets onto a shell surface. We find that droplets spread out to $\sim 5$ times their original diameter on the surface of a plasma-etched $\mathrm{CH}$-shell. Using this measurement, we have succeeded in dispensing linearly connected PA droplets along a shell's equator. We have achieved a degree of control not obtainable in any of our prior work. These results are summarized in figures 5 and 6.

Figure 1 shows our experimental set-up. It consists of an piezoelectric-driven, drop-on-demand dispenser unit mounted on a 3-axis translation stage, a variable RPM stepper motor for single-axis rotation of stock-mounted shells, and a CCD camera coupled to a far-field microscope used to observe drop-formation (with an LED strobe) and their subsequent deposition onto a shell surface.

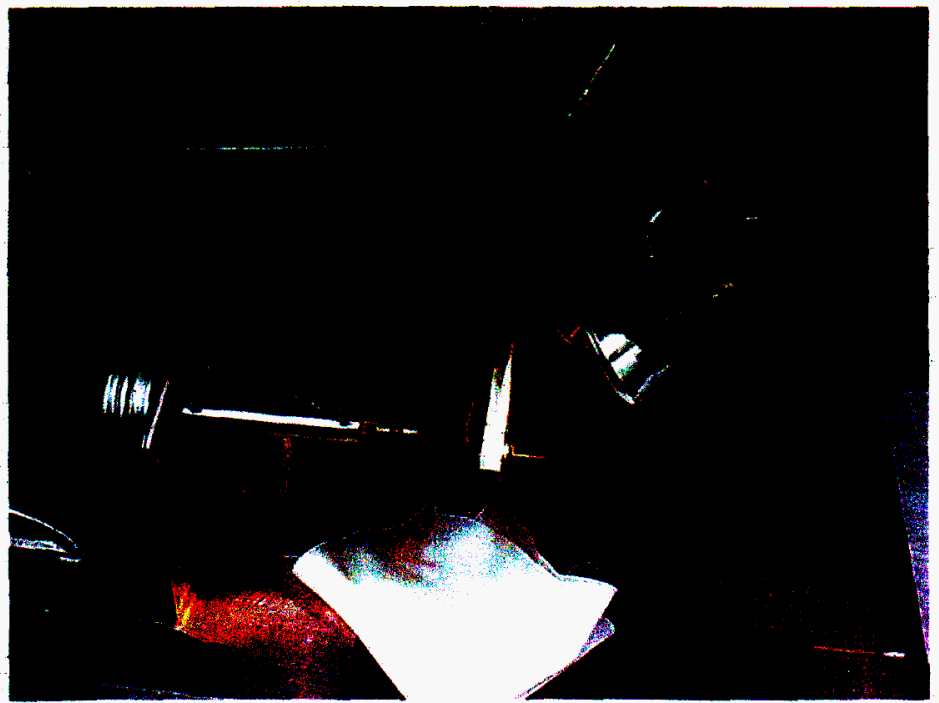

Figure 1: Test Setup

The dispenser gravity-feeds PA from a syringe reservoir. This reservoir must be placed such that the PA meniscus in the reservoir is $\sim 2 \mathrm{~cm}$ below the dispenser's glass tube exit-tip. This establishes a hydrostatic pressure in the fluid necessary for correct operation of the dispenser. This produces a slight positive curvature in the fluid meniscus at the exit-tip which inhibits air-bubbles from entering the tip as drops are driven out by electromechanically induced pressure waves. 
Pressure waves in the fluid are induced by electrically stimulating a lead zirconate titanate (PZT) material surrounding the glass tube. This generates an acoustic/pressure wave in the fluid. Arrival of this wave at the exit-tip ejects a single drop. This mode of drop formation is termed drop-on-demand. This same device can produce continuous drop formation by pressuring the fluid (as described) and electrically stimulating the PZT material with a time-varying electrical signal.

The form and magnitude of this signal is critical for successful formation of single and continuous drops-see figure 2-which is produced by a drive-electronics unit controlled by a computer via serial communication.

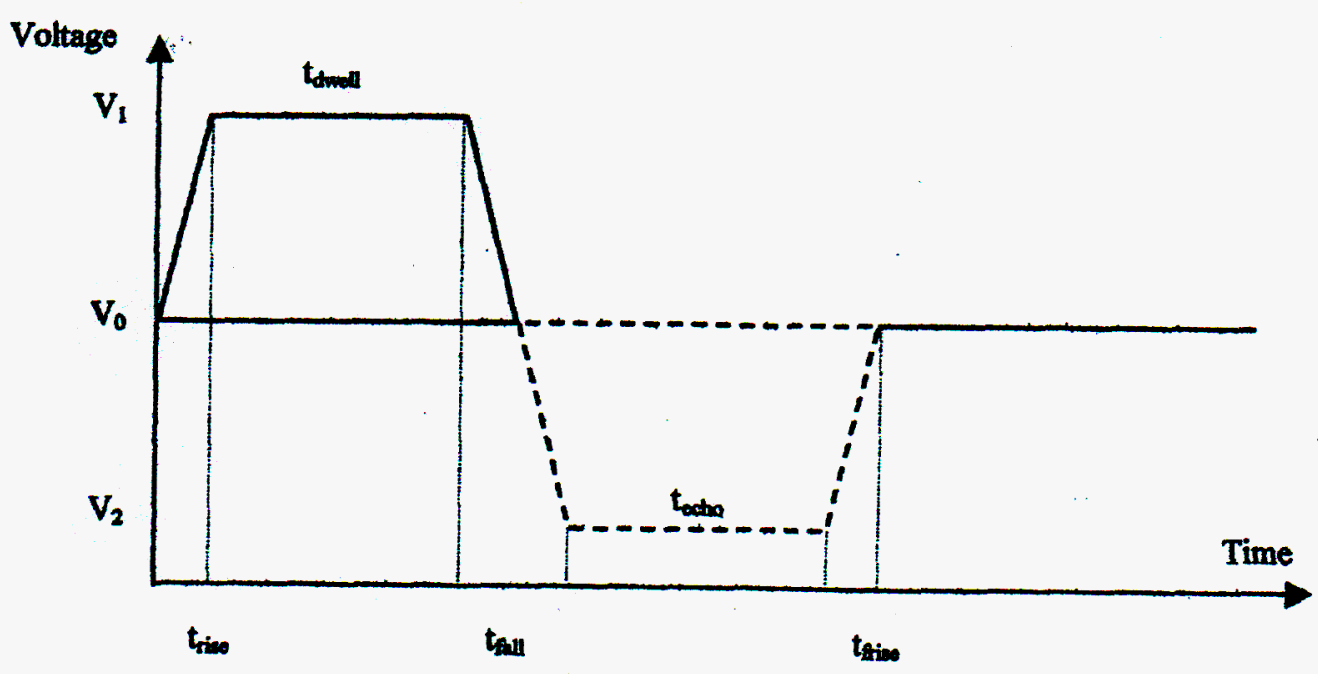

Figure 2: driving waveform of the plezoelectric actuator

Waveform Parameters are:

$t_{\text {rise }}=$ initial rise time

$t_{\text {dwell }}=$ time at $V_{1}$ (high voltage)

$t_{\text {fall }}=$ transition time from $V_{1}$ to $V_{2}$ (low voltage)

$t_{\text {echo }}=$ time at $V_{2}$

$t_{\text {trise }}=$ final rise time

Following the manufacturer's (MicroFab Technologies Inc.) recommendations:

$V_{0}=0$ and $V_{1}=-V_{2}$ 
The first three images of figure 3 are stroboscopic photomicrographs of the formation of a single droplet of NMP, with $v \sim 1.7 \mathrm{cp}$ (reported). Droplets are formed at a frequency of $30 \mathrm{~Hz}$ with the dispenser running in continuous mode. In other words, the stream of drops is continuous, but using a synchronized strobe and delay, we are able to view different droplet formation time-slices. The image on the lower right indicates a phenomenon that usually results with too much drive voltage. It is undesirable because this produces fluctuating, multiple droplet-streams.

Applying these same techniques to a $3 \%$ solution of PA (using a different pulse waveform) result in similar photomicrographs-see figure 4. PA behaves differently due to an increase in $v$ and density of the fluid. To determine $v, a$ Gilmont falling-ball viscometer was employed and a series of measurements taken. These measurements give $v \sim 16.5 \mathrm{cp}$, which is less than half the maximum operating range of the dispenser. This suggests a higher \%PA concentration may be used, minimizing the amount of NMP needed to evaporate between PA coats, while maximizing the thickness per coat. Waveforms for $100 \%$ NMP and 3\% PA solutions are summarized in table 1.
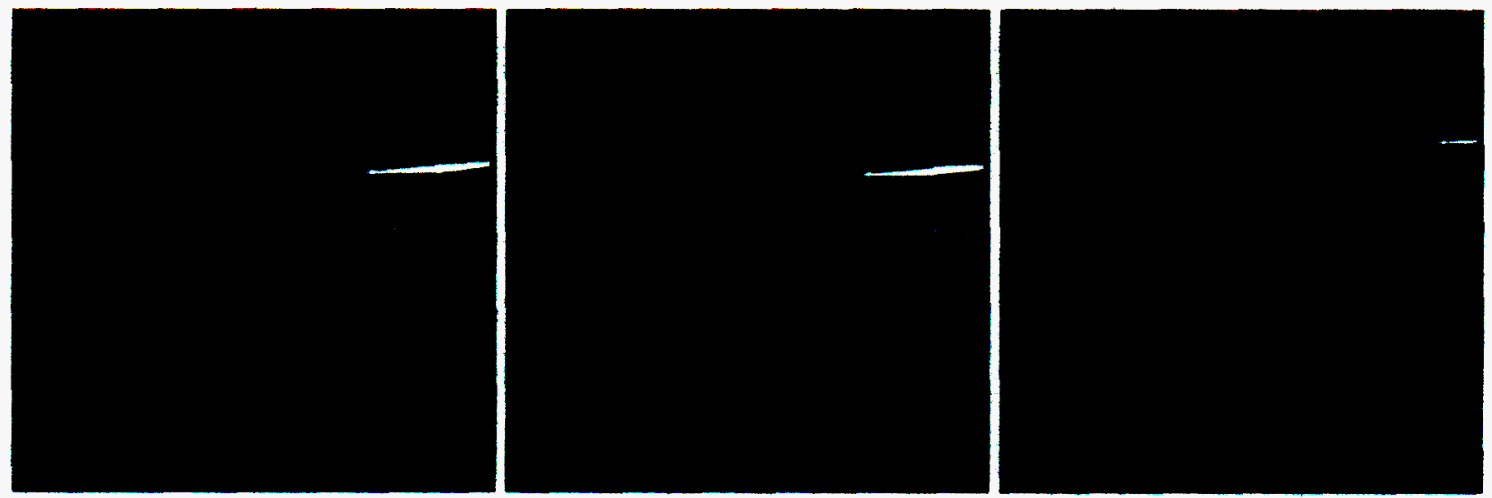

Figure 4: 25\% PA solution showing Rayleigh Instability breakup and final drop formation

\begin{tabular}{|l|l|l|}
\hline & $100 \%$ NMP & $3 \%$ PA / 97\% NMP \\
\hline$t_{\text {rise, }} \mu \mathrm{sec}$ & 3 & 2 \\
\hline$t_{\text {cwell }}$ & 8 & 50 \\
\hline$t_{\text {tall }}$ & 3 & 2 \\
\hline$t_{\text {echo }}$ & 17 & 100 \\
\hline$t_{\text {rise }}$ & 3 & 2 \\
\hline$V_{1}=-V_{2}, D C$ & 85 & 65 \\
\hline
\end{tabular}

Table 1: waveform parameters

Luxel Corporation

Friday Harbor, WA 98250
4

Master Task Agreement No. MTA B514570 
$t_{\text {rise }}=t_{\text {fall }}=t_{\text {frise }} \sim 3 \mu$ s to minimize the drive voltage, $V_{1}$

towell is approximated by the following relation:

$$
\mathrm{t}_{\mathrm{dwell}}=\frac{2 L}{c},
$$

where $L \sim 13 \mathrm{~mm}$ is the distance between the middle of the PZT element to the glass tube tip and $c$ is the speed of sound in the fluid. For our $\sim 3 \%$ PA $\sim 93 \% \mathrm{~N}$ Methyl-2-pyrrolidone (NMP) solution we approximated c to be that in water at $20^{\circ}$ C, i.e. $c \sim 1480 \mathrm{~m} / \mathrm{s}$. A more accurate measure of $c$ may be obtained from the bulk-modulus, which we have yet to measure, and the density of the solution.

By using our far-field microscope, digital camera, frame-grabber board, and a strobe-driven LED, we are able to witness drop creation, formation, and separation from the dispenser tip-see figures 3 and 4 . This visual information facilitates optimization of drop-creation and geometry by varying (1) the waveform parameters $V_{1}, t_{\text {rise, }} t_{\text {dwell, }} t_{\text {fall }}, t_{\text {echo, }}$, and $t_{\text {frise }}$ and (2) fluid pressure. Each fluid has different requirements based on their physical properties, including $v$ (absolute viscosity), and wetting behavior within the dispenser. The dispenser is designed to expel fluids having $v$ between $\sim 1$ and $\sim 40 \mathrm{cp}$.
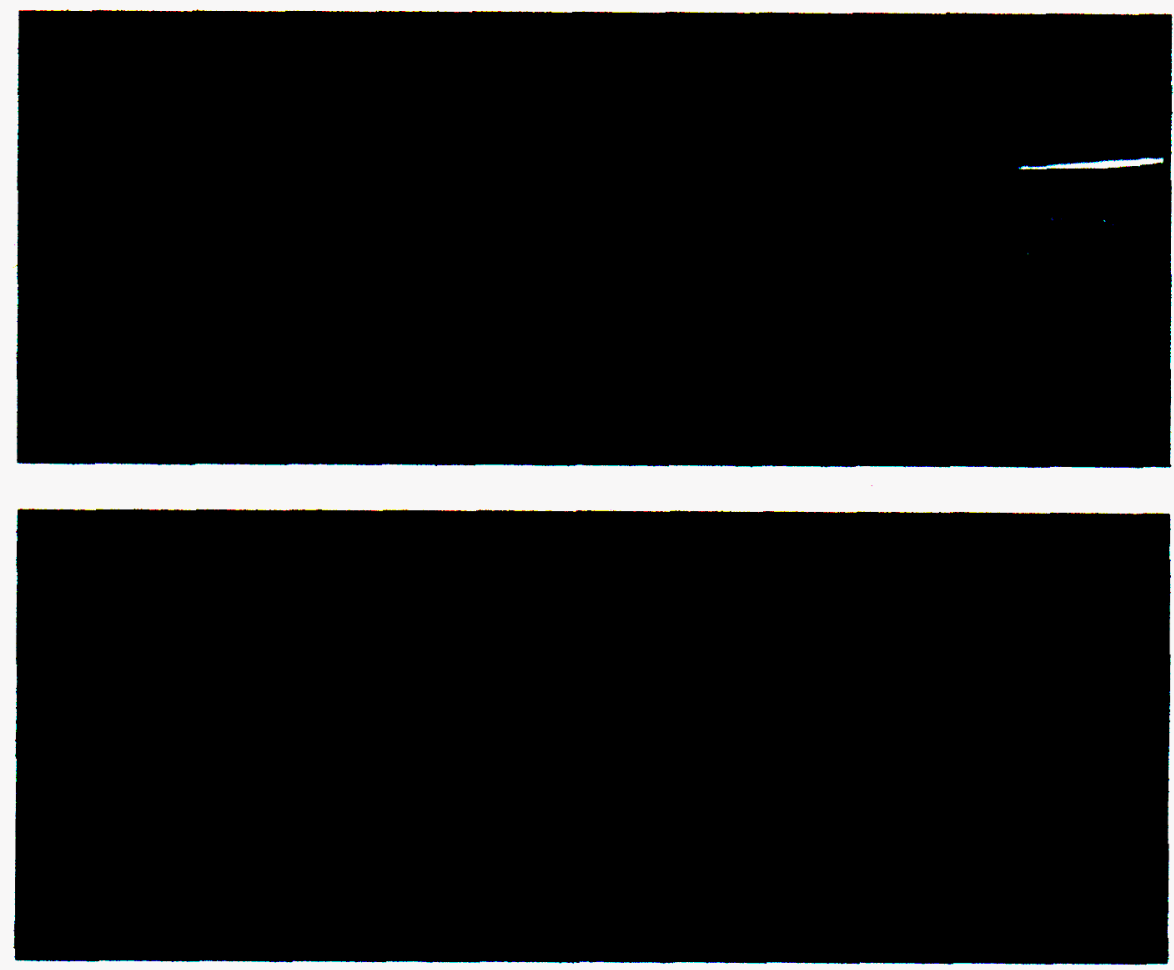

Figure 3: NMP droplet formation, break up due to Rayleigh instability, and droplet separation. Lower-right shows an undesirable condition: drop with a satellite 
After determining the pulse waveform needed to drive uniform and consistent droplets of $3 \%$ PA, the next step was to observe the behavior of the droplets on a $\mathrm{CH}$-shell surface. Assuming $50 \mu \mathrm{m}$ diameter droplets with a trajectory perpendicular to the axis-of-shell-rotation and no spreading on the shell surface, the drop-frequency required for a connected line of PA droplets around the equator of a $1 \mathrm{~mm}$ radius shell rotating at 30 RPM is obtained as follows.

From the frame-of-reference of a moving droplet, the velocity of the shell surface at the equator is the shell's equatorial tangential velocity, $v_{\text {tangential }}=r \omega$, where $r$ and $\omega$ are the shell's radius and angular velocity, respectively. In a time $\Delta t$, the surface moves a distance, $\Delta x \approx v_{\text {tangential }}{ }^{*} \Delta t$. Equating $\Delta x$ to the drop-diameter, $\sigma$, gives the time, $\Delta t$, needed for the surface to traverse one drop diameter: $\Delta t=\sigma$ $/ v_{\text {tangential. }} \Delta t$ is also the drop-period needed to form a connected line of $\sigma$ diameter drops around the equator. Hence the drop-frequency, $f$, is:

$$
\mathrm{f}=\frac{1}{\Delta t}=\frac{v_{\text {tan gential }}}{\sigma}=\frac{r \omega}{\sigma}
$$

Using the values given above (after converting RPM to radians per second) in Eq. 1 gives $f \sim 63 \mathrm{~Hz}$. Given that the shell rotates once per 2 seconds, a total of $f$ * $2 \mathrm{sec}=126$ drops are needed. Running the dispenser in a 126-drops/triggermode, we triggered it once at the calculated $f$.

The resulting deposition on the plasma-etched surface indicates considerable spreading and droplet overlap due to impact and wetting--see left image of figure 5. A second experiment was then performed to determine the spreading of a single, $50 \mu \mathrm{m}$ diameter droplet on the equatorial surface.

Triggering the dispenser at $\mathrm{f}=1 \mathrm{~Hz}$ and 2 drops/trigger, produced 1 droplet per hemisphere. This allowed measurement of the wetting-diameter and tested the control of droplet placement. Results are indicated in the right-side image of figure 5 . Measurement of the spread drop gives a wetting diameter of $\sim 225 \mu \mathrm{m}$, i.e. 5-times its original diameter. Although not observable in Figure 5's rightside image, the second drop is clearly observed to be rotated $\sim 180^{\circ}$ relative to the first drop along the equator.

Luxel Corporation

Friday Harbor, WA 98250 

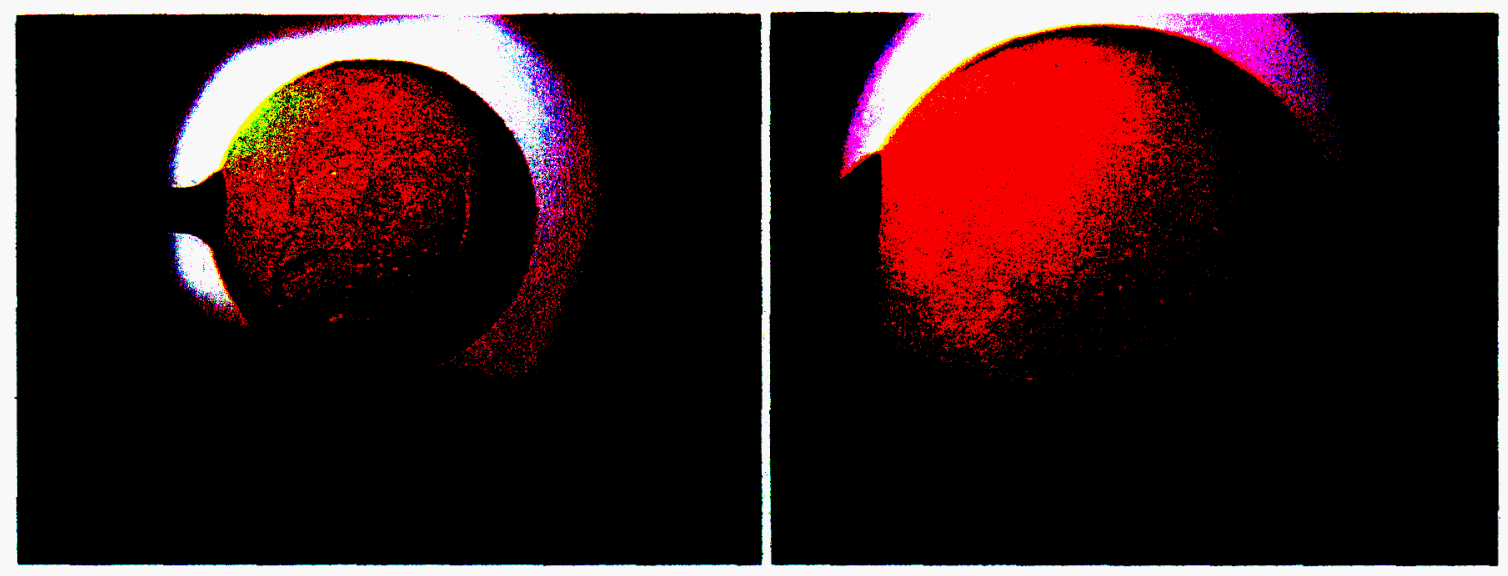

Figure 5: Left: PA swath on a CH-shell. Right: Image of a single droplet

Given the measured wetting-diameter, a third experiment was performed too see if we could produce a continuous line of minimally connected drops. Using $\sigma \sim$ $225 \mu \mathrm{m}$ in Eq. 1 results in $f \sim 14 \mathrm{~Hz}$. Results using this drop-frequency are shown in figure 6. A linear series of connected drops are clearly seen, indicating that our measured wetting-diameter is reasonably correct and that droplet placement on the shell surface is precisely controlled.

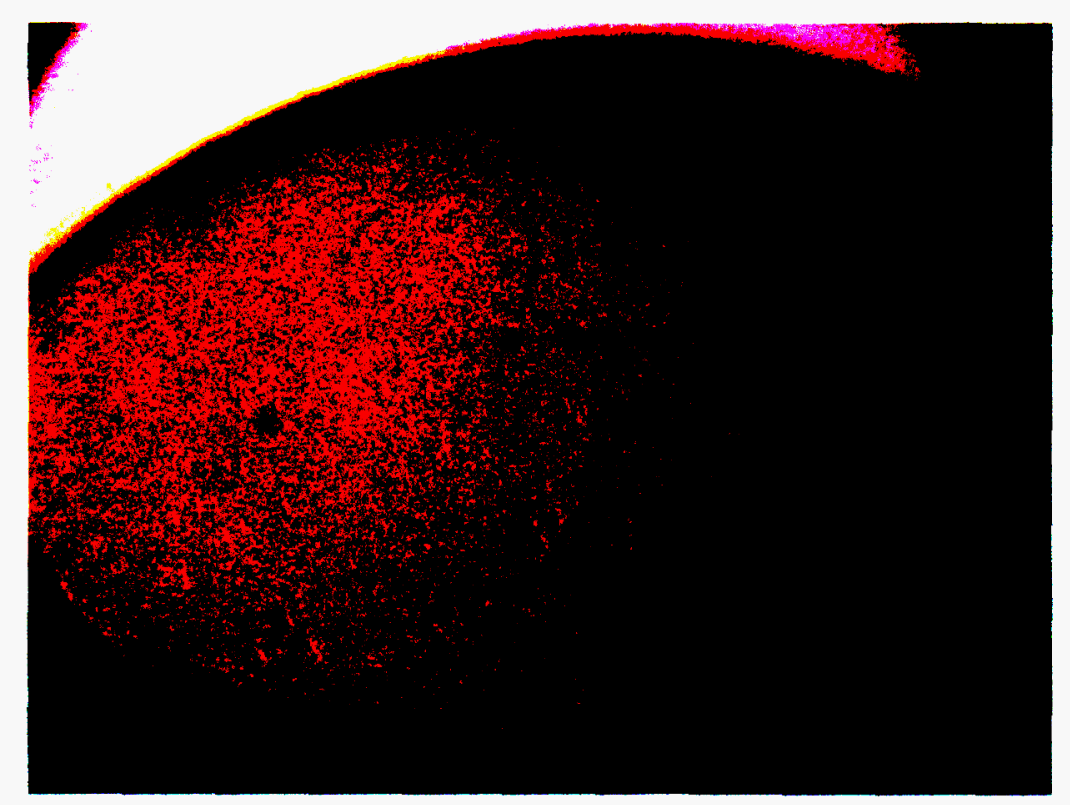

Figure 6: Image showing precise placement of $\sim 50 \mu \mathrm{m}$ diameter droplets initially spaced $\sim 225 \mu \mathrm{m}$ apart.

Given these results, the following empirical calculations are performed to approximate several useful results applicable to a bare, plasma-etched $\mathrm{CH}$-shell.

Given a shell rotating once per 2 seconds and that $\sim 14$ wetted, minimallyconnected drops are needed to trace-out half the circumference of a shell, the 
total time needed to for a single, complete coating is $\sim 28$ seconds. Given the surface area of a CH-shell is $4 \pi \mathrm{r}^{2} \sim 1.3 \times 10^{7} \mu \mathrm{m}^{2}$ and the area of a wetted-drop is $\pi \mathrm{r}^{2} \sim 4.0 \times 10^{4} \mu \mathrm{m}^{2}$, the total number of drops needed to completely cover a shell is $\mathrm{N} \sim 325$ drops. Given that we have measured the density of a $3 \%$ PA / $97 \%$ NMP solution to be $\rho_{P A} \sim 1.1 \mathrm{~g} / \mathrm{cm}^{3}$ and the volume of a $\sim 25 \mu \mathrm{m}$ radius drop is $(4 / 3) \pi r^{3} \sim 6.5 \times 10^{-8} \mathrm{~cm}^{3}$, the mass per drop is $m_{\text {drop }} \sim 7.2 \times 10^{-8} \mathrm{~g}$. Assuming that a single drop spreads out as a cylinder with average height, $d$, then the thickness of a single, wetted-drop is $d=m_{\text {drop }} /\left(\rho_{\mathrm{PA}} \pi r^{2}\right) \sim 1.3 \mu \mathrm{m}$. The total solution mass of a covered shell is $m_{\text {solution }}=N^{*} m_{\text {drop }} \sim 2.3 \times 10^{-5} \mathrm{~g}$. Given these approximations, the average thickness of this solution layer is approximated as follows.

The volume contained between two shells with $r_{0}<r_{1}$ is

$$
V=\frac{4}{3} \pi\left(r_{1}^{3}-r_{0}^{3}\right)=\frac{m}{\rho} .
$$

Solving for $r_{1}$ gives

$$
r_{1}=\left(\frac{3 m}{4 \pi \rho}+r_{0}^{3}\right)^{\frac{1}{3}}
$$

The average thickness between the two shells is therefore

$$
\Delta r=r_{1}-r_{0}=\left(\frac{3 m}{4 \pi \rho}+r_{0}^{3}\right)^{\frac{1}{3}}-r_{0}=r_{0}\left(\frac{3 m}{4 \pi \rho r_{0}^{3}}+1\right)^{\frac{1}{3}}-r_{0} \approx r_{0}\left(1+\frac{m}{4 \pi \rho r_{0}^{3}}\right)-r_{0}=\frac{m}{4 \pi \rho r_{0}^{2}},
$$

(Eq. 2)

where the (binomial expansion) approximation is valid for $m \leq 5 \mathrm{mg}$.

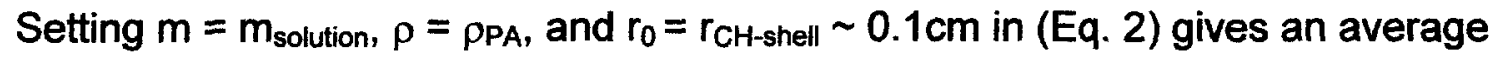
thickness of $\Delta \mathrm{r}_{\text {solution }} \sim 1.6 \mu \mathrm{m}$. This is comparable to the cylindrical approximation of the average thickness of a single, wetted-drop calculated above.

Finally, if $\sim 97 \%$ of the solution is NMP and all of this is evaporated during a standard high-temperature cure, producing $P$ (polyimide), then $\sim 3 \%$ of $m_{\text {solution }}$ remains. Setting $m \sim 0.03{ }^{*} m_{\text {solution, }} \rho=\rho p \sim 1.4 \mathrm{~g} / \mathrm{cm}^{3}$ (reported), and $r_{0}=r_{\mathrm{CH}}$-shell in (Eq. 2) gives an average thickness of $\Delta r_{p} \sim 39 \mathrm{~nm}$. If this thickness applies to subsequent cured layer-thicknesses of PA deposited on soft-baked PA, then (Eq. 2) predicts $\sim 3.8 \times 10^{3}$ layers are needed to attain a $P$ wall-thickness of $150 \mu \mathrm{m}$.

The significance of this last prediction is questionable considering that our microdispensing device handles viscosities up to $\sim 2.4$ times greater than what is used 
in this calculation. This implies that using a higher viscosity PA solution will reduce the number of required layers to meet the $150 \mu \mathrm{m}$ thickness requirement. Also, we have yet to determine how many layers can or need to be added before soft-baking. Finally, the number of layers needed to get a smooth surface using the surface tension effect is yet to be determined.

The following is a discussion of related developments regarding current and future investigations.

As proposed, radiant heating as well as convective soft-bake heating will be used. We have acquired an infrared (IR) lamp, which uses an elliptical reflector to focus energy onto a 1/4" spot. This has been incorporated into a closed-loop control system using a $0.005^{\prime \prime}$ diameter thermocouple (TC) for input and verified for temperature response and control. Initial tests with the IR lamp are promising for the application of soft-bake cycles. Furthermore, since the maximum attainable temperatures of this lamp are reported to be well above $P$ cure temperatures, it should be able to imidize PA on $\mathrm{CH}$-shells given an inert atmosphere.

A long-standing question has been why do stock-mounted, PA-coated $\mathrm{CH}$-shells tend to collapse when soft-backed with a halogen reflector lamp or the above IR radiation heater, while soft-baking with a Radak-oven convective heater does not tend to cause collapse. A partial answer seems lie in the difference between how and where convective and radiant heating couples energy to a PA-layer and the underlying $\mathrm{CH}$-shell. Convective heating produces masses of excited gas molecules that subsequently couples energy only to the PA-surface via collision of fast-moving molecules with the surface, hence collisional-surface-heating. A fraction of this energy is then transferred inward through the PA-layer to the $\mathrm{CH}$ shell via conduction. Radiation heating simultaneously couples energy "instantly" throughout the PA-layer and underlying $\mathrm{CH}$-shell via photons. Excited molecules through-out then transfer energy via conduction to surrounding molecules.

This suggests that if we are to successfully soft-bake and possibly imidize with a radiation source, we need to couple energy only to the PA-layer, as in the collisional-surface-heating case. A reasonable way to do this is by filtering the radiation source, such that only a narrow-band of photon energies tuned to significant PA adsorption-bands, is allowed to "illuminate" the PA-layer. An LLNL absorption spectrum of $3 \%$ liquid and soft-baked PA indicates that it absorbs energy significantly at $2.8,3.4$, and $5.9 \mu \mathrm{m}$. During the next work phase we plan to locate and acquire an IR-filter meeting this band-pass.

The MicroJet has performed quite well for the tests reported here, however it is an extremely delicate device. Extreme care must be employed while mounting the device and priming or purging fluid to expel air bubbles. Although we are using a $0.2 \mu \mathrm{m}$ filter, small particles find their way to block the jet's $50 \mu \mathrm{m}$ orifice, which we can directly observe with our imaging system. Additionally, large air

Luxel Corporation

Friday Harbor, WA 98250
8

$$
\begin{array}{r}
\text { Master Task Agreement No. MTA B514570 } \\
\text { Prnaress Rennrt } 2032301
\end{array}
$$


bubbles cause blockage, which remain until back purged. These hurdles have been overcome by experience operating the device. A new concern is that PA and/or NMP seems to be 'attacking' the subcomponents of the MicroJet. Even though the actual jet mechanism is glass, it is built of other small parts held in place by adhesive. In fact, our last two jets appeared to suffer from this chemical attack, rendering them inoperable. The manufacturer, MicroFab Industries, has been contacted and a team of their $R$ \& $D$ personnel is examining solutions based on our suggestions. The anticipated solution should not impact jets performance.

We have procured most of the experimental equipment necessary to produce $>50 \mu \mathrm{m}$ thick coatings by April 20th, 2001. It remains to be shown if we can produce thickness non-uniformities less than $1 \mu \mathrm{m}$, over a length of $100 \mu \mathrm{m}$ at the equator of stalk mounted mandrels.

A summary of activities planned for the remaining portion of Task $A$ follows.

1. Finalize the new clean room and transfer our current equipment into that area. All new equipment recommendations have been finalized and ordered. Remaining equipment is near quoted delivery date. A new experimental setup will be assembled in the clean room and made ready for the automated drop-on-demand deposition process of PA onto spherical mandrels.

2. Broaden the area of uniform coverage to include more than just the equatorial region of the mandrel. This will be accomplished by using a single-axis micro-positioner stage to move the jet along the axis-of-rotation by (1), varying the translational velocity of the inkjet while maintaining constant droplet frequency or by (2), varying the droplet frequency while maintaining constant translational velocity.

3. Continue to optimize single-droplet PA deposition for uniform thickness, coverage, and smoothness.

4. Establish repeatable and reliable setup procedures for the MicroJet dispenser to eliminate daily setup time and fine-tuning.

5. Work with MicroFab to modify the fabrication of the MicroJet to resist degradation of the jet components due to the PA solution.

6. Investigate the use of IR radiation to soft-bake and imidize P-coated $\mathrm{CH}$ shells

7. Provide LLNL with soft-baked and imidized P-coated $\mathrm{CH}$-shell samples for surface analysis. 
Third Progress Report:

NIF Polyimide Ablators Produced by Solution Spray Techniques

Utilizing our new class $100 / 1000$ clean-room and a revised experimental setup, we have focused on establishing a piezo-electric inkjet system to coat plasmaetched, stock-mounted mandrels with PA (polyamic acid). We are currently able to dispense on-demand, individual $\sim 50 \mu \mathrm{m}$ (micrometer) diameter, linearly connected PA ( $3 \%$ by weight) droplets, laid down into bands about the shell surface. This technique is then expanded upon to interconnect the bands into a continuous coating covering a $\sim 90$ degree sector of the shell surface (Fig. 3 ). Furthermore, we have accomplished preliminary droplet deposition onto a levitating shell using our ink-jet (MicroJet) delivery system and an acoustic levitator (Fig. 5).

Figure 1 shows a photomicrograph in which we succeeded in placing a series of precisely spaced, $50 \mu \mathrm{m}$ diameter PA droplets about the equator. Also shown is the same shell after a subsequent heating process in which a continuous built-up polyimide coating with a thickness of $\sim 200 \mathrm{~nm}$ per soft bake cycle resulted. Assuming droplets in the band spread to $\sim 225 \mu \mathrm{m}$ upon impact, $\sim 28$ wetted, minimally connected drops are needed to trace-out the circumference of a $2 \mathrm{~mm}$ diameter shell. This resulted in producing continuous, interconnected single PA layers $\sim 1.6 \mu \mathrm{m}$ thick.
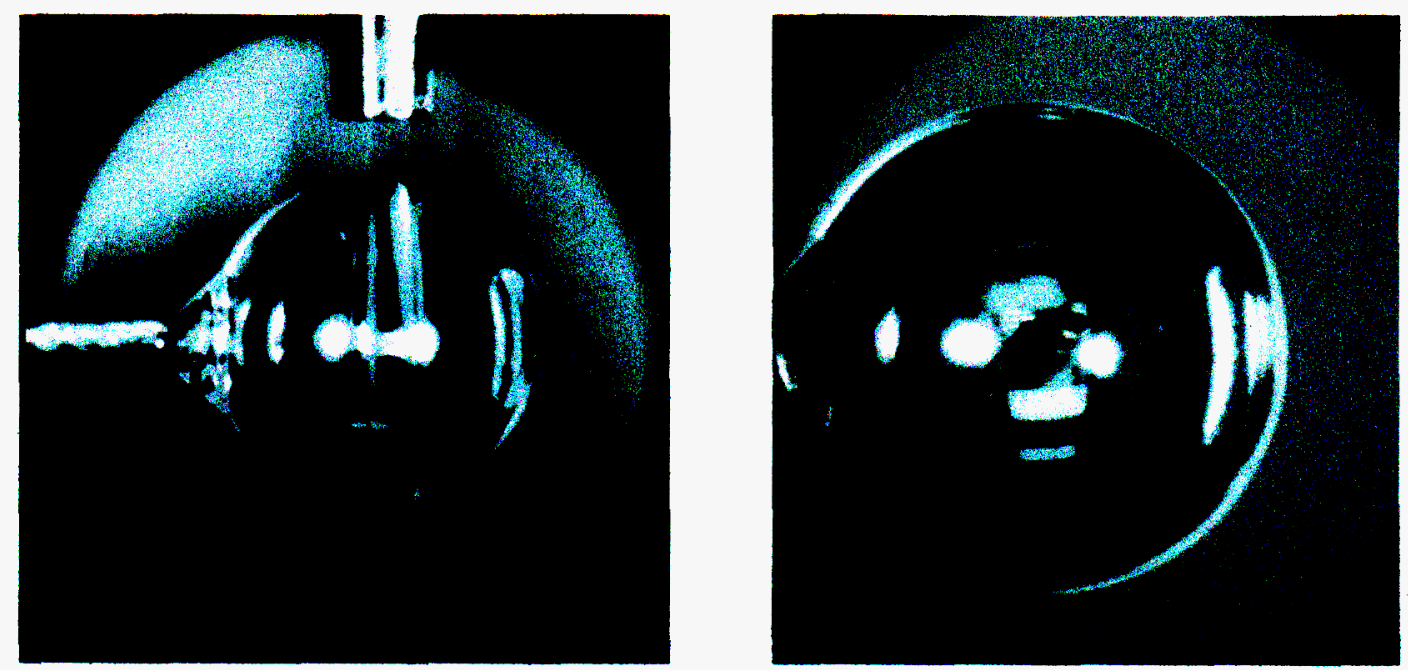

Figure 1: Equatorial PA Deposition about a CH shell and Subsequent SoftBake 
Continuing with this effort we succeeded in equatorially coating a $\mathrm{CH}$ shell with 40 layers of PA. After every $5^{\text {th }}$ PA layer-deposition, the shell was soft-baked. The finished specimen was then forwarded to LLNL for analysis.

Figure 2 shows the patch power spectra as provided by LLNL. They are calculated from three equatorial scans (View 1, 2 \& 3) and one bare-shell scan (taken at the shell pole). The NIF power spectra standard is also shown for reference. Views 1 and 3 (green and gold circles) illustrate that the coating has the effect of smoothing the higher frequency bumps on the mandrel. View 2 (green circles) has one large $4 \mu \mathrm{m}$ bump in the field of view, which has the effect of pumping up the high frequency end of the power spectrum.

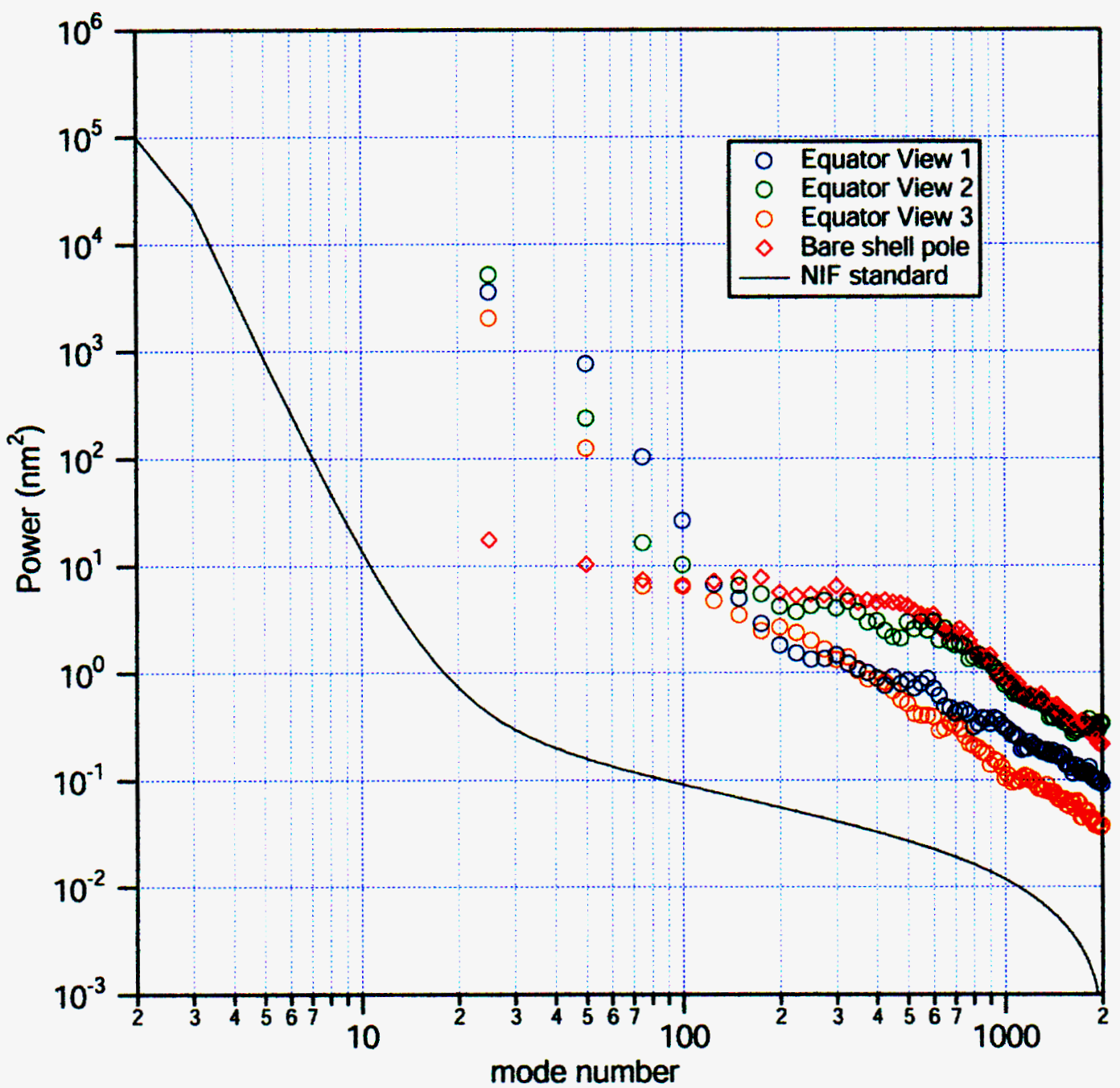

Figure 2: Patch Power Spectra for $\mathbf{4 0}$ layers of PA Applied at Equator 
The equatorial set-up was modified to direct droplets normal to the shell surface at any angular position, allowing for multi-band deposition. This was accomplished by rotating the microjet about the shell centroid using a rotational stage for precise angular positioning $\left(\geq 1.8^{\circ}\right)$. Software was written to simultaneously control (1) the stage position and (2) the number and frequency of discrete PA droplets, both of which depend on the jet's angular position. This modified experimental setup is indicated in Figure 3.

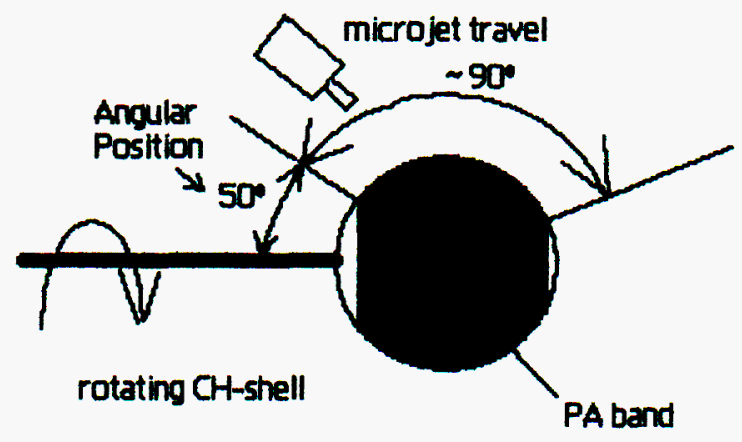

Figure 3: Multi-Band Angular Deposition Set-up

Given a $\sim 225 \mu \mathrm{m}$ arc-length or $\sim 12.6^{\circ}$ step over a $90^{\circ}$ sector, the following input parameters (table 1) are needed to cover the defined band shown in figures 3 and 4.

\begin{tabular}{|c|c|c|c|c|}
\hline$\frac{\text { Angular }}{\text { position }}$ & $\frac{\text { Radius }}{\underline{\mu}}$ & $\begin{array}{l}\text { Number } \\
\text { of Drops }\end{array}$ & $\frac{\frac{\text { Drop }}{\text { Frequency, }}}{\mathrm{Hz}}$ & $\frac{\text { Time }}{\text { constant }}$ \\
\hline $\begin{array}{c}50 \\
63 \\
75 \\
88 \\
100 \\
113 \\
126 \\
138\end{array}$ & $\begin{array}{l}766 \\
890 \\
969 \\
999 \\
979 \\
910 \\
794 \\
639\end{array}$ & $\begin{array}{l}21 \\
25 \\
27 \\
28 \\
27 \\
25 \\
22 \\
18\end{array}$ & $\begin{array}{c}11 \\
12 \\
14 \\
14 \\
14 \\
13 \\
11 \\
9\end{array}$ & $\begin{array}{r}2 \\
2 \\
2 \\
2 \\
2 \\
2 \\
2 \\
2\end{array}$ \\
\hline
\end{tabular}

Table 1

Using these parameters, we applied twenty-eight PA coatings onto a shell, which was soft-baked after coat \#10,20, and 28 . This resulted in a visually-uniform coating that confirmed our deposition parameters, revealing good wet-out between droplets and adjacent bands. Further deposition onto this shell was discontinued due to MicroJet failure. Replications of this experiment produced 
equivalent deposition behavior, however none of the completed specimens were deemed of quality to submit for surface characterization. why?
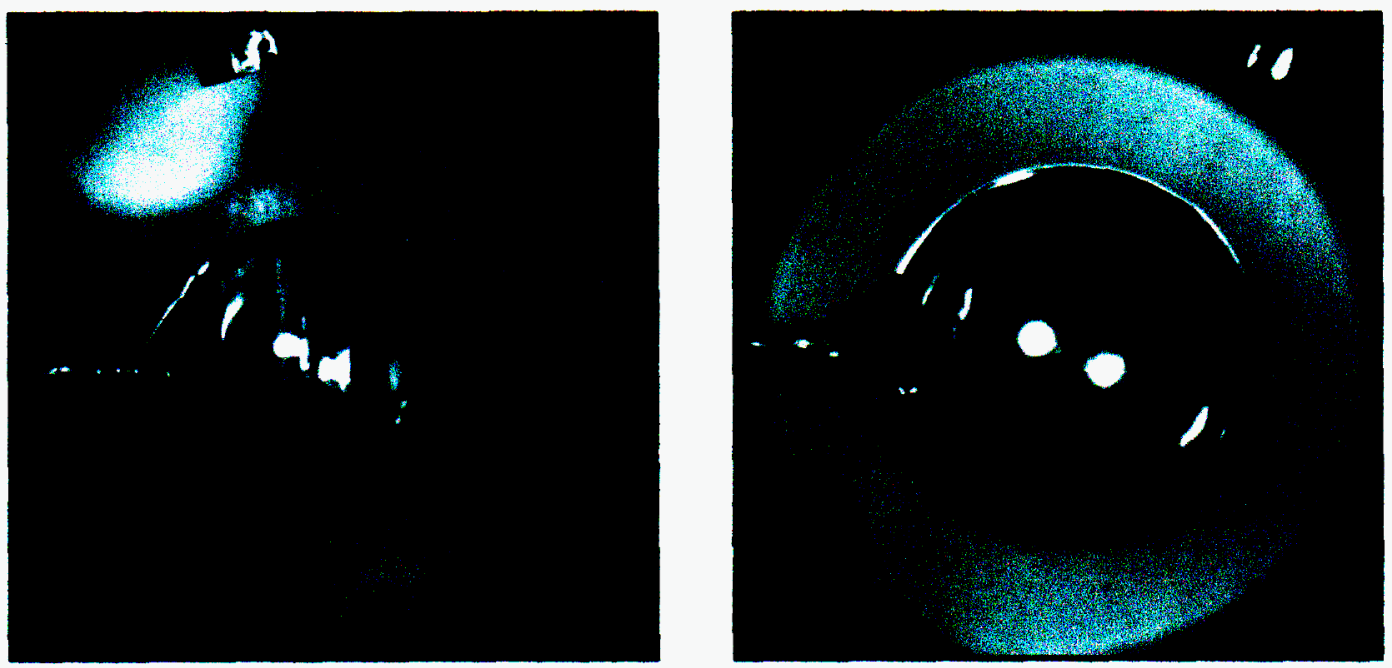

Figure 4: Placement of continuous PA bands, 28 layers total, soft-baked every 10 layers. Note flowing together of single droplets.

During this same reporting period, droplet deposition onto acoustically levitated shells was revisited using lessons learned from stalk-mounted experiments. We observed new behavior when attempting to soft-bake levitating shells with a coilheater.

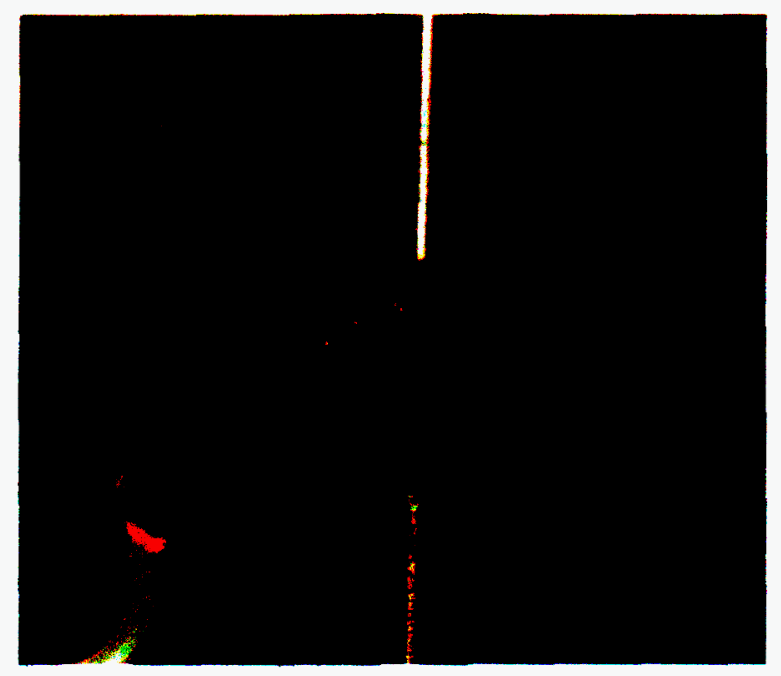

Figure 5: CH Shell in position for PA Deposition

The operating parameters for the W-375 Sonicator(3) were established to consistently position a $\mathrm{CH}$ shell in the acoustic field. Using our single-axis system, this resulted in a position roughly $1 / 3$ down from the passive reflector 
that still provided enough clearance to approach the shell within $2-3 \mathrm{~cm}$, which was adequate for deposition. Using our far-field microscope, test firings of the microjet indicated the acoustic field was not deflecting PA droplets. Furthermore, the shell was not forced out of levitation due to the droplets' momentum, as confirmed by a previous momentum-transfer calculation. This shell maintained its relative position whether firing a single, isolated droplet or a series of droplets at $60 \mathrm{~Hz}$.

Rotation and oscillation of the shell within the acoustic field is sensitive to drop frequency and placement. However, given this sensitivity, and our ability to control mass, velocity and position of droplets, it is possible to calculate the change in angular momentum as droplets are deposited.

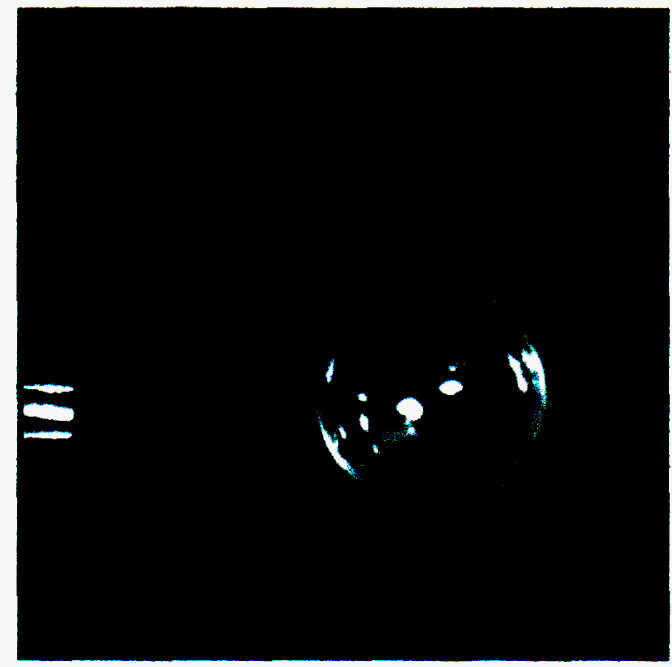

Figure 6: Photomicrograph of rotating/levitating $\mathrm{CH}$ Shell with $\sim 2700$ droplets of PA.

After an initial firing of droplets aimed at a fixed location, a great circle of drops forms, causing the shell to rotate about a constant axis-of-rotation at a rate believed to correlate to the drop frequency. Continued deposition eventually causes the shell to shift to a new and constant axis-of-rotation, thus indicating a change in angular momentum. Also, during deposition, as droplets are applied to the shell, an increase in mass is indicated by an observed downward displacement. This begins to occur at $\sim 500$ drops $(\sim 36 \mu \mathrm{g})$. Increasing the power output of the ultrasonic transducer compensates for this minor downward displacement. However, it was also observed that increasing the mass by $\sim 5000$ droplets of PA $(\sim 400 \mu \mathrm{g})$ caused the shell to fall out of single-axis levitation.

A modified method for convective/IR heating was investigated on a levitating shell that utilized a custom-fabricated open-ended heater capable of emitting energy in the IR region of interest. Initial results indicate that, regardless of heat, the placement of the one-inch internal diameter, cylindrical IR emitter source 
around the levitating sphere significantly modifies the spatially-extended acoustic field. Changing the mass distribution and geometry about the levitation space is observed to cause this effect. The IR emitter will be rebuilt and radially enlarged to a 2-inch inner diameter to minimize acoustic-field interference.

The following is a discussion of related developments regarding current and future investigations.

As proposed, radiant heating as well as convective soft-bake heating will be used. The implementation of an infrared (IR) blackbody emitter with a matched wavelength to the absorption wavelength of PA is a worthwhile approach as confirmed by published findings. LLNL absorption spectra for $3 \%$ liquid and softbaked PA indicate good mid-IR absorption at 2.8,3.4, and 5.9um. Further published findings indicate more imidization-activity occurs at $\sim 3 \mu \mathrm{m}$, even though PA has less absorption at that wavelength than at $\sim 6 \mu \mathrm{m}$. The advantages of tuned IR-curing over convective curing are:

1. Significantly reduced soft-bake time from $\sim 1$ hour to several minutes.

2. Volume heating homogeneously deposits energy throughout, giving better morphology of the cured substrate.

The calculated IR spectral density plots (figure 7) for an ideal blackbody emitter indicated virtually no mid-IR emission at $100^{\circ} \mathrm{C}$ and little mid-IR emission at 300 ${ }^{\circ} \mathrm{C}$. This implies that the dominant heat-transfer mode for the $100 / 300^{\circ} \mathrm{C}$ imidization heat-cycle is convection, with virtually no radiant component. 


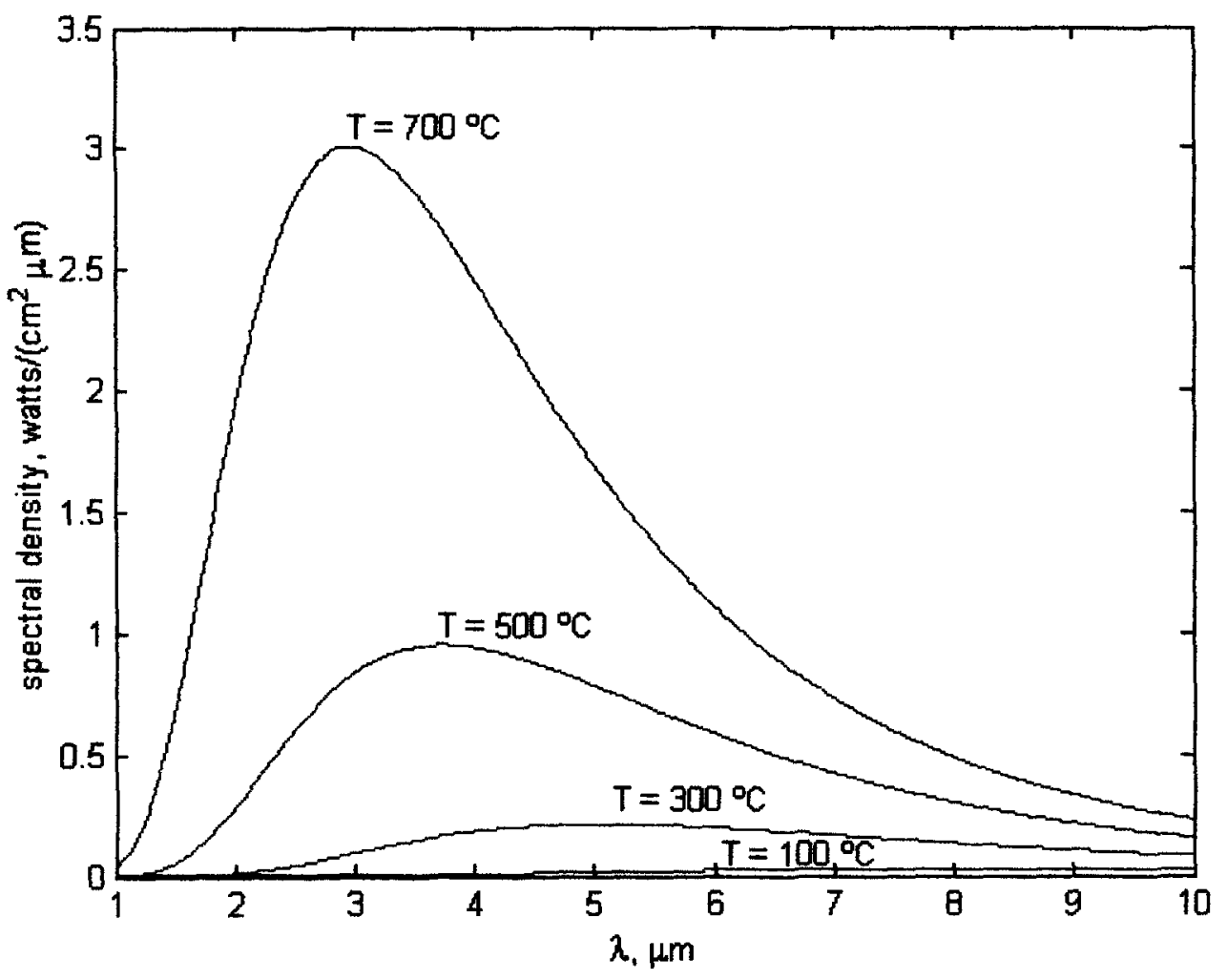

Figure 7: Black Body Spectral Density

Tuned IR-curing will require increasing the heater temperature to between 500 and $1000^{\circ} \mathrm{C}$. This will require some experimentation and precise temperature measurements of the shell and the emitter using an embedded thermocouple. The advantage of higher blackbody temperatures is that the heat transfer rate significantly increases as the temperature difference between the power source and the substrate source increases. This is shown by the equation for radiative heat transfer,

$$
Q / A=F_{v}{ }^{*} \varepsilon^{*} \sigma^{*}\left(T_{s}^{4}-T_{t}^{4}\right),
$$

where $\mathrm{Q} / \mathrm{A}=$ heat flux density, watts $/ \mathrm{cm} 2$, and

$$
\begin{aligned}
& F_{v}=\text { field of view (ratio), } \\
& \varepsilon=\text { emissivity of source (ratio), } \\
& \sigma=\text { Stefan-Boltzman constant, } \\
& T_{s}=\text { temperature of power source in Kelvin }(K), \text { and } \\
& T_{t}=\text { temperature of target substrate in } \mathrm{K} .
\end{aligned}
$$

The required IR filter characteristics may be derived by from the spectral density plot of a custom-fabricated heater. By using a mid-IR blackbody power source, 
relatively little short-IR $(\leq 2 \mu \mathrm{m})$ is generated. Therefore, using a short-pass optical filter to reject long-IR wavelengths $(\geq 3 \mu \mathrm{m})$ may be sufficient. Using the long-wavelength cut-off of quartz $(\sim 3 \mu \mathrm{m})$ or sapphire $(\sim 6 \mu \mathrm{m})$, a short-pass filter will offer significant long-IR wavelength heat rejection.

Published findings indicate that a black-body temperature of $700^{\circ} \mathrm{C}$ produces the spectral density required for rapid ( minutes), volumetric ( $10 \mu \mathrm{m}$ thickness) imidization. However, attaining sufficient power at mid-IR wavelengths may require increasing the heater-element temperature to $\sim 1000^{\circ} \mathrm{C}$, such that the absorbed, long-IR photons will appreciably heat the quartz or sapphire filter, producing convective heating. Quartz tolerates temperatures $\leq 1000^{\circ} \mathrm{C}$. Given this case, a cylindrical quartz or sapphire vacuum bottle may be used to further reject the generated heat.

There are several IR pyrometers that could be obtained for measuring the shell surface temperature. However, until a suitable IR wavelength is determined for the IR emitter, we will hold off on this procurement, as the instruments are tuned to specific wavelengths.

The MicroJet has performed relatively well for the results reported here, however we have learned that it is a 'finicky' device requiring significant user-intervention for proper and reliable operation. A growing concern, previously reported, is the PA-NMP mixture appears to chemically 'attack' the MicroJet's subcomponents.

Although we think the actual jet mechanism of glass surrounded by a piezoelectric element is chemically inert, they are held together by adhesives that seem to degrade rapidly from PA-NMP exposure. Our last several jets appear to suffer from this attack, rendering them inoperable after $\sim 1$ week of operation. The manufacturer, MicroFab Industries, is aware of this problem, but has yet to produce a solution. We are striving to solve this problem by modifying working jets to minimize adhesive-exposure and thereby increasing longevity.

We are also investigating another other ink-jet manufacturer, InkJet Technology, who claims their inkjets are chemically resistant to PA-NMP mixtures. They are currently testing their inkjet's compatibility with our mixture.

The experimental equipment necessary to produce $>50 \mu \mathrm{m}$ thick coatings by 31 May 2001 are in place. However, it remains to be shown if we can produce thickness non-uniformities less than $1 \mu \mathrm{m}$, over a length of $100 \mu \mathrm{m}$ at the equator of stalk mounted mandrels.

A summary of activities planned for the remaining portion of Task $A$ follows:

Luxel Corporation

Friday Harbor, WA 98250
4/20/2001

8 Master Task Agreement No. MTA B514570 
1. Continue to optimize and automate PA deposition and soft-bake cycles on a stalk-mounted shell such that $>50 \mu \mathrm{m}$ thick uniform coating can be completed. This will be accomplished by using our existing single-axis rotational stage to orientate the jet about the shell centroid while performing successive coatings interspaced with soft-baking.

2. Continue to establish repeatable and reliable setup procedures for the inkjet dispenser to eliminate daily setup time and fine-tuning.

3. Continue to work with MicroFab, Inc. to modify the fabrication of the MicroJet to resist degradation of the jet components due to the PA/NMP solution.

4. Initiate contacts with alternative manufacturers regarding more durable, robust ink-jet dispensers. Procure loaner-equipment to evaluate performance.

5. Investigate the use of wavelength specific IR radiation and emitter geometry to soft-bake and imidize $\mathrm{P}$-coated $\mathrm{CH}$-shells.

6. Provide LLNL with soft-baked and imidized P-coated CH-shell samples for surface analysis. 


\section{Fourth Progress Report:}

\section{NIF Polyimide Ablators Produced by Solution Spray Techniques}

Using our rotational, drop-on-demand piezo-electric deposition system, we are dispensing $\sim 80 \mu \mathrm{m}$ (micrometer) diameter, PA (polyamic acid, $3 \%$ by weight) droplets into $\sim 225 \mu \mathrm{m}$-wide bands to cover $\sim 75 \%$ of a rotating, plasma-etched, stock-mounted mandrel. Although production of characterization-grade specimens has recently been hampered by an unexplained degradation of PA liquid in the deposition system, we have overcome this setback, gaining a better understanding of the deposition system. Additionally, all of the delivery system tubing has been replaced with PFA Teflon ${ }^{\circledast}$. Design, fabrication, and preliminary tests of a blackbody, selective IR ( 1 to $3 \mu \mathrm{m}$ ) furnace has been completed. Initial results indicate that PA-coated mandrels soft-bake in $>1$ order-ofmagnitude less-time than that required by our convective furnace.

Our general coating technique is to rotate the PA dispenser through $\sim 90^{\circ}$ arc, and at calculated positions, deposit a continuous band that subsequently interconnects to adjacent bands, thus producing a uniform coating. Initially, we deposited a ring of minimally-connected PA drops per band, i.e. one coat per band. This process was repeated enough times to cover a mandrel with a backand-forth motion. However, it was found that these minimal bands where dryingout prior to subsequent coatings, resulting in reduced wetability between individual drops and bands-see Figure 1 . We modified our deposition technique to deposit multiple coats per band before coating adjacent bands. This significantly enhances continuity between drops and bands-see Figures 2 and 3.
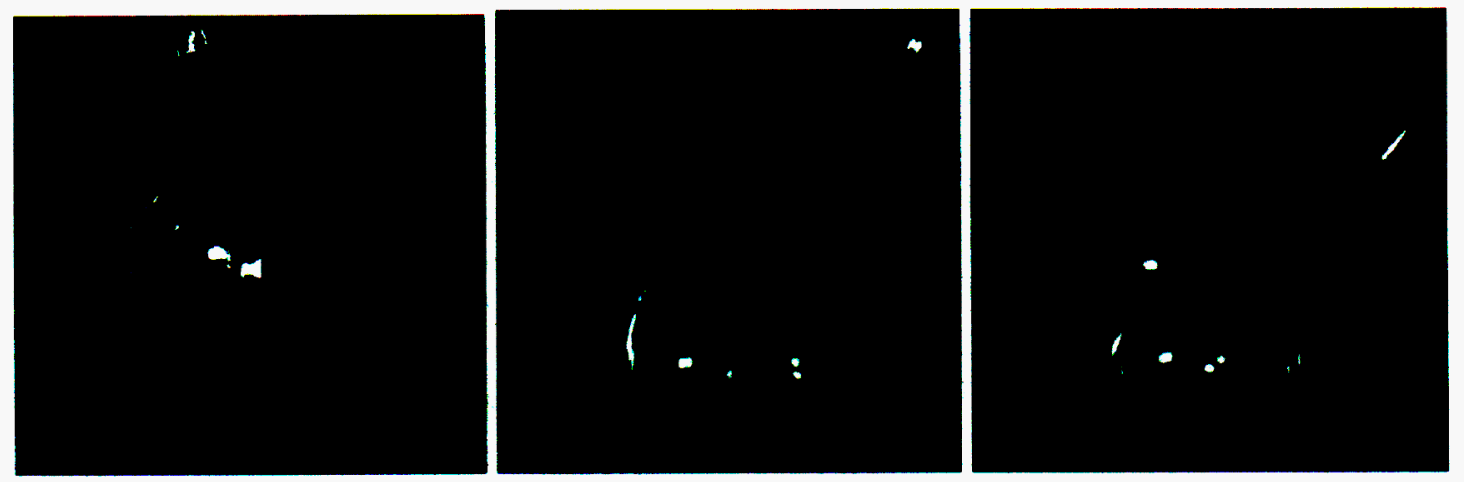

Initial (Figure 1) and Current (Figures 2, \& 3) Deposition Techniques

While the deposition/coating process is now reliable, the production of surface characterization quality shells has been hampered by a chemical interaction between the PANMP (N-Methyl-2-pyrrolidone) solution and one or several of the 
inner-surface tubing materials. This produced soft-baked specimens with a cloudy/speckled surface micro-structure having a $\sim 40 \mu \mathrm{m} /$ bump spatial bumpperiod with $\sim 0.5 \mu \mathrm{m}$ high bumps. An exhaustive step-by-step investigation was therefore conducted. Segment-by-segment the system's fluid delivery components and the PANMP solution ingredients were analyzed, characterized, and/or replaced in search of the root cause. The assumed-proven delivery system from the reservoir to the ink-jet dispenser used C-Flex ${ }^{\oplus}$ tubing, which has physical properties similar to silicone with the chemical compatibility of Tygon ${ }^{\varpi}$. After removing this tubing, the above-described phenomenon stopped occurring. Passing the solution through this section of tubing only, caused the phenomenon to reappear. This indicates that $\mathrm{C}-\mathrm{Flex}^{\oplus}$ is not chemically compatible with PANMP. All tubing in our deposition-system has since then been upgraded to high-purity PFA Teflon ${ }^{\circledast}$, i.e. high-density plastic. The net result of this investigation is that after intense scrutiny of each component, we have reinforced our procedures and equipment needed for cleaning, operating, and dropoptimizing the MicroJet ${ }^{\oplus}$ dispenser. While periodic mechanical failure of a few jets has occurred, and is inevitable, we are now confident in the mechanics of depositing onto stalk-mounted shells with this dispenser.

A custom fabricated 1.4-inch inner diameter, open-ended heater comprising of a double quartz cylinder surrounded by resistance coils and a silicon carbide matrix was used to conduct initial IR (infra-red) soft-bake trials on test specimens (Fig. 4). The quartz cylinder is a band-pass optical filter up to $3 \mu \mathrm{m}$, while providing convective heat rejection by evacuation of the air space between the cylinder walls. Tests indicate a minimum $200^{\circ} \mathrm{C}$ convective temperature reduction between the emitter and the core due to the quartz vacuum sleeve. An insulating blanket improves furnace efficiency and helps reduce convective air currents.

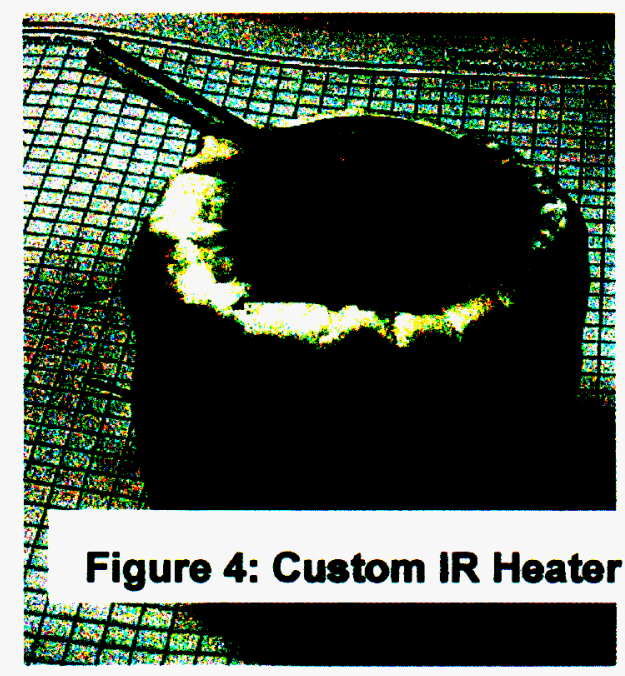

Luxel Corporation

Friday Harbor, WA 98250
$6 / 15 / 2001$

2
Task Order No. B514571

Master Task Agreement No. MTA B514570 
Previous investigations suggested that to achieve proper spectral heating at PANMP's $3 \mu \mathrm{m}$ absorption wavelength, a temperature of $700^{\circ} \mathrm{C}$ would be required. Initial trials with the black-body emitter operating at $700^{\circ} \mathrm{C}$ and the core temperature at $-450^{\circ} \mathrm{C}$ proved much to great a power density for the coated shells. Subsequent trials at a reduced emitter temperature of $300^{\circ} \mathrm{C}$ and a core temperature of $-100^{\circ} \mathrm{C}$ proved more controllable and provided the necessary energy to evaporate the NMP solvent. Qualitative observations that sufficient soft-baking occurs in $\sim 45$ seconds. This is a significant reduction in the time required for the overall deposition process. Time-Temperature-Mass experiments will be conducted to solidify the process before carrying out IR softbaking on characterization-quality shells. Two areas remain to be explored regarding IR heating: 1) the impact of IR volume heating on thick-coated shells, i.e., less soft-bake time for greater volume; and 2) a short dwell time may be required after IR immersion to allow residual radiation effects to relax before continued deposition.

The experimental equipment and techniques necessary to produce $>50 \mu \mathrm{m}$ thick coatings by 30 June 2001 are in place. Incorporation of IR heating into the coating cycle is a near-term reality and production and delivery of surface characterization quality shells will commence immediately.

A summary of activities planned for the remaining portion of Task A follows:

1. Continue to optimize, establish repeatable and reliable setup procedures, and automate PA deposition and soft-bake cycles on a stalk-mounted shell such that $>50 \mu \mathrm{m}$ thick uniform coating can be completed.

2. Continue to work with MicroFab, Inc. to improve the reliability i.e., robustness of the MicroJet ${ }^{\circledR}$ to resist degradation of components due to the PANMP solution.

3. In parallel, procure alternative ink-jet dispenser from InkJet Technology, Inc., to complete evaluation of a more durable, robust ink-jet dispenser.

4. After additional testing, incorporate wavelength specific IR radiation and emitter geometry to soft-bake and imidize P-coated $\mathrm{CH}$-shells.

5. Provide LLNL with soft-baked and imidized $\mathrm{P}$-coated $\mathrm{CH}$-shell samples for surface analysis. 


\section{Fifth Progress Report:}

\section{NIF Polyimide Ablators Produced by Solution Spray Techniques}

Since the last review, we have abandoned the rotational deposition system in favor of a simplified linear system, conducted over twenty full-coverage, thick coating experiments, and have incorporated an IR (infra-red) soft-bake process that, based on initial tests, was deemed acceptable to investigate at this time. The temptation to perform successive coatings in minutes rather than hours was difficult to ignore, and as now realized, coating cycle times including soft-bake have averaged approximately $51 / 2$ minutes. Consequently, as coating cycle times have drastically reduced from the incorporation of IR curing, new 'dragons', possibly from IR radiation side-effects, have emerged that have impacted low mode surface characteristics. Although many samples show regions of coating success, it has been rather unfortunate that we have had once again difficulty in producing widespread, defect-free quality specimens suitable for LLNL analysis. The specimens we have produced however, have provided other valuable information regarding droplet flow behavior, process time studies, observation of stalk-shell epoxy interactions pertaining to full coverage coats, and IR process and geometry developments adaptable to acoustic levitation. We were also able to take advantage of a SEM (scanning electron microscope) in an attempt to verify thickness calculations on a multi-coated, convectively soft-baked specimen.

After several months of operational experience using our rotational, drop-ondemand piezo-electric deposition system, we realized we were subjecting the one component of our system to an environment that is the least forgiving to rapid accelerations. The MicroJet ${ }^{\oplus}$ dispenser is now stationary and fixed in orientation. The rotating stalk-mounted $\mathrm{CH}$-shell is now translated by a linear stage across the path of the ejected droplets of liquid PA (polyamic acid, $3 \%$ by weight). A modified computer program using previous parameters was developed to discretely position the shell at band intervals coinciding with a $\sim 225 \mu \mathrm{m}$ drop spread and changing radius of the shell. The ability to dispense $\sim 80 \mu \mathrm{m}$ (micrometer) diameter, PA droplets into $\sim 225 \mu \mathrm{m}$-wide bands covering at least $75 \%$ of a $\mathrm{CH}$ mandrel has not been compromised by the new setup, and the elimination of the non-normal trajectory of droplets at the shell surface seems to have little influence in the deposition process. Additionally, all of the delivery system tubing has been replaced with semi-rigid PFA Teflon ${ }^{\oplus}$. The improved setup and chemically inert tubing greatly eliminates chances for contamination and provides a fluid path of minimal resistance from the reservoir to the dispenser. The linear deposition setup including placement of the IR furnace is shown in Figure 1. 


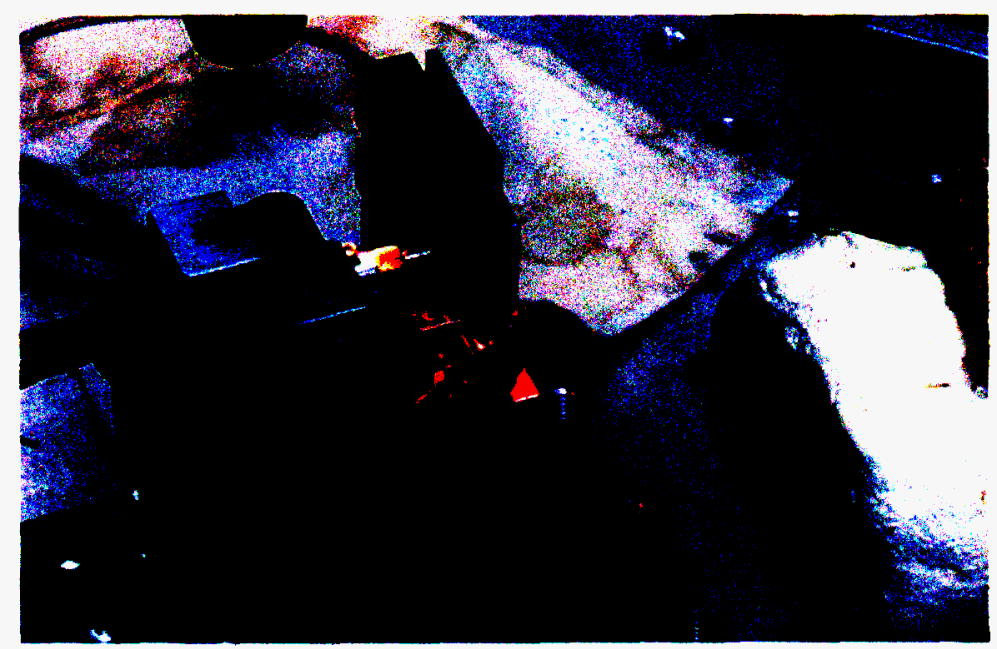

Figure 1: Custom IR furnace and linear deposition setup

Observations using the linear droplet deposition system show similar droplet placement ability as the rotational system. Figure 2 depicts the droplets as they strike the surface and their continued application until the desired wet thickness is reached. The number of wet coats per band, and the droplet band width per a given cycle or event, can be controlled within the program. Figure 2 also shows the relative size of a PA droplet. Recently, using comparative measurements between a known dimensioned feature and applying an image pixel ratio against the droplet, a diameter of $\sim 80 \mu \mathrm{m}$ has been determined. The original assumption of $\sim 50 \mu \mathrm{m}$ was based on the dispenser orifice. Individual drop volume is now calculated at $268 \rho \mathrm{pL}$ (picoliters), a four-fold increase over the previous volume estimate of $65 \mathrm{\rho L}$ per droplet. This is a significant increase in the amount of PA originally thought to be wet-deposited upon the shell surface
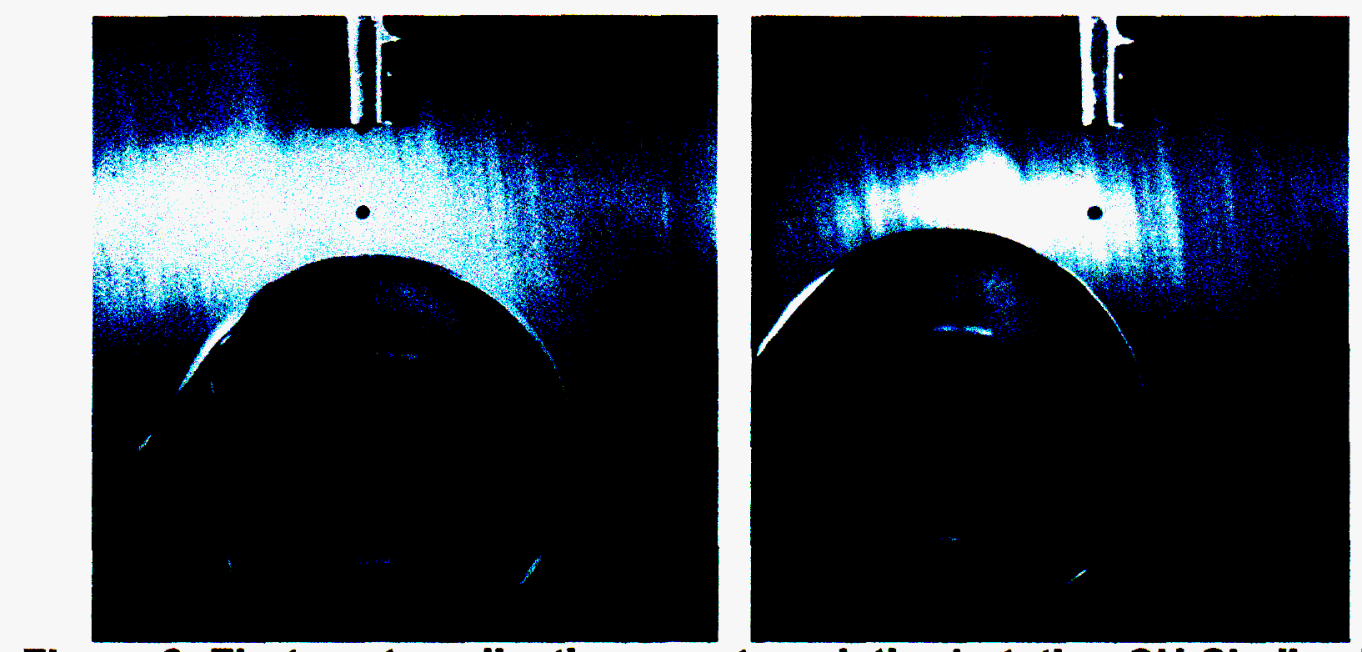

Figure 2: First coat application on a translating/rotating CH-Shell using stationary dispenser and linear deposition 
While the deposition/coating process is now more reliable, and we are now confident in the procedures and equipment needed for cleaning, operating, and drop optimizing the MicroJet ${ }^{*}$ dispenser, the production of defect-free specimens has been slowed by a non-uniform wetness condition. This condition appears to be exacerbated by IR radiation effects from the previous soft-bake process. Although, small regions upon the shell surface are worthy of characterization, the shell as a whole does not represent the surface characteristics necessary for a worthy PSD analysis. Figures 3 and 4 show the increasing roughness typical of a multi-coat IR soft-baked specimen.

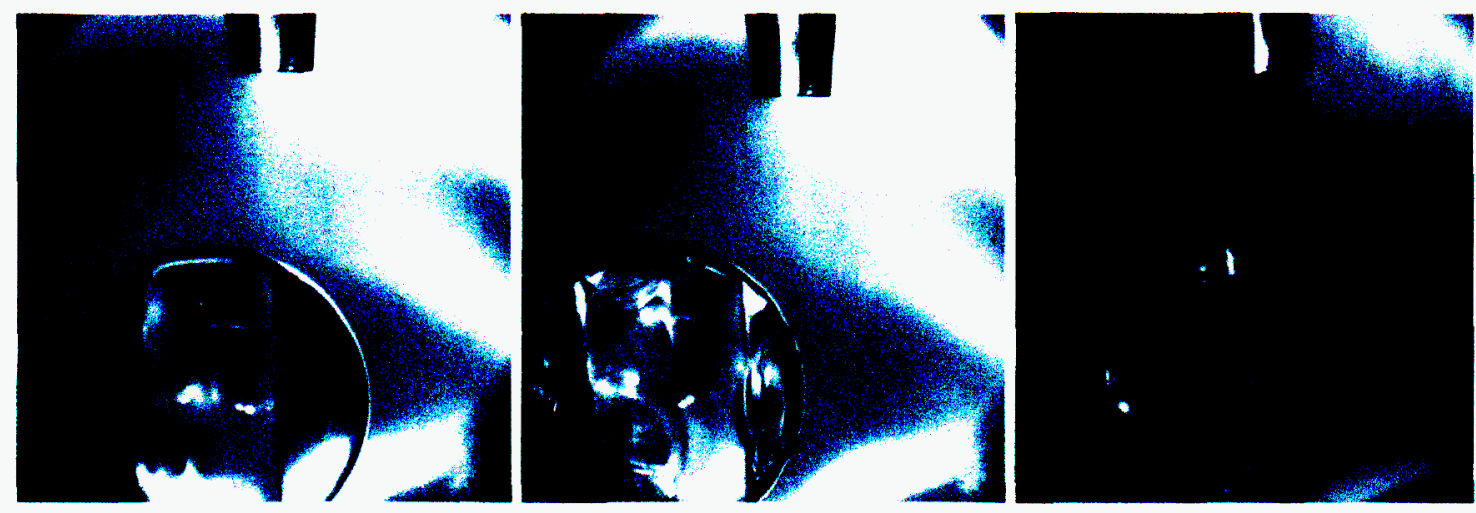

Figure 3: A multi-coated CH-shell during deposition and interspersed by soft-baked IR cycles. Image 1 is initial cycle of 2 coats. Image 2 is the second cycle consisting of 2 coats after $1 \mathrm{~m} 15 \mathrm{~s}$ IR immersion. Image 3 is the $10^{\text {th }}$ cycle consisting of 3 coats after $1 \mathrm{~m} 15 \mathrm{~s}$ IR immersion.

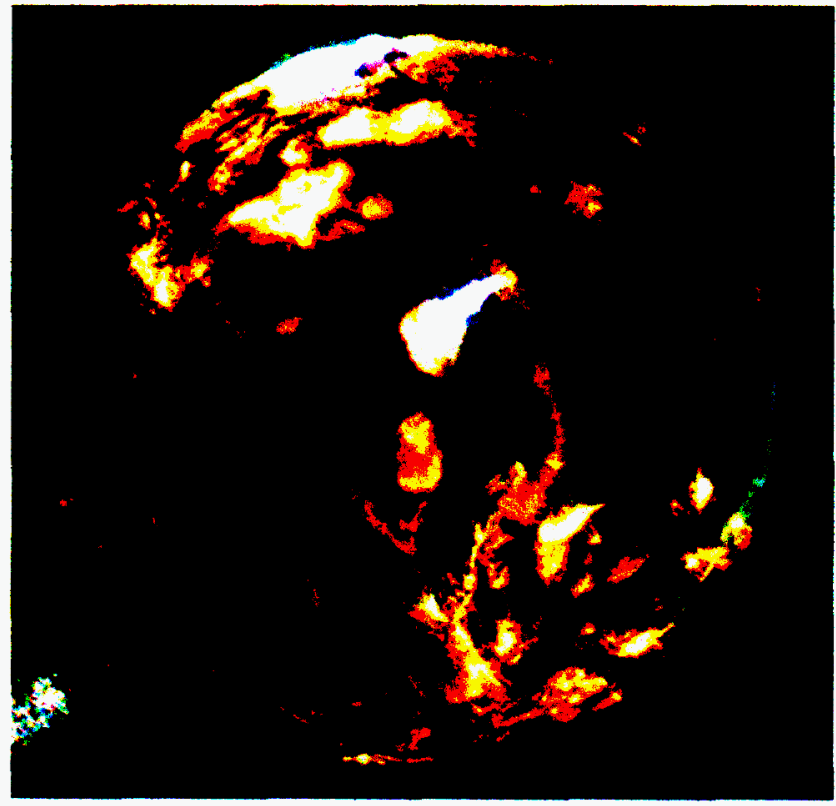

Figure 4: Finished sample from above after 20 IR cycles and a total application of 70 wet coatings. 
Although the last five multi-coated coated specimens IR soft-baked were less than optimum, our custom quartz/silicon carbide 1.4-inch I.D., open-ended IR heater has performed true to design. Previous investigations suggested that to achieve proper spectral heating at PANMP's $3 \mu \mathrm{m}$ absorption wavelength, a temperature of $700^{\circ} \mathrm{C}$ would be required. Subsequent trials at a much reduced emitter temperature of $300^{\circ} \mathrm{C}$ and a core temperature of $\sim 100^{\circ} \mathrm{C}$ proved more controllable and provided the necessary energy to evaporate the NMP solvent. Qualitative observations suggested that sufficient soft-baking occurs in $\sim 45$ seconds. However, for the soft-bakes performed on recent samples, an emitter temperature of $280^{\circ} \mathrm{C}$ was used which again resulted in a core temperature of $\sim 100^{\circ} \mathrm{C}$. The soft bake time was then adjusted to 75 seconds. Average coating and soft-bake cycle times averaged $51 / 2$ minutes. At this rate, it was possible to coat a specimen with $\sim 70$ coats in about 2 hours. However, as mentioned in the last report, before the IR process is deemed reliable and repeatable, at least two areas must be further explored: 1) the potential impact of IR volume heating on thick-coated shells, i.e., less soft-bake time for greater volume; and 2) a short dwell time ( $\sim 5$ minutes) will probably be required after IR immersion to allow residual radiation effects to relax before continued deposition.

We recently examined a multi-PA coated, convectively soft-baked $\mathrm{CH}$-shell using an JEOL JSM-35 SEM. The specimen was truncated and the remnants were mounted to a stub and gold-palladium sputtered before examination. The cross sections of the $\mathrm{CH}$-shell wall were scrutinized for evidence of a soft-baked $\mathrm{P}$ coating upon the outer surface-see images in Figure 5 . The outer surface of the coated shell is upward in the two images. In the left image, the P layer is shown to extend out beyond the underlying $\mathrm{CH}$-shell layer, which is indicated by the lower step (bounded by the first and second white lines from the bottom. The $\mathrm{CH}$ layer indicates a thickness of $\sim 15 \mu \mathrm{m}$. The right image is a magnification of the $P$ layer, which has $70 P$ coats. This layer has an indicated thickness of $\sim 10 \mu \mathrm{m}$. This is consistent with our theoretical thickness calculation of $\sim 11 \mu \mathrm{m}$.
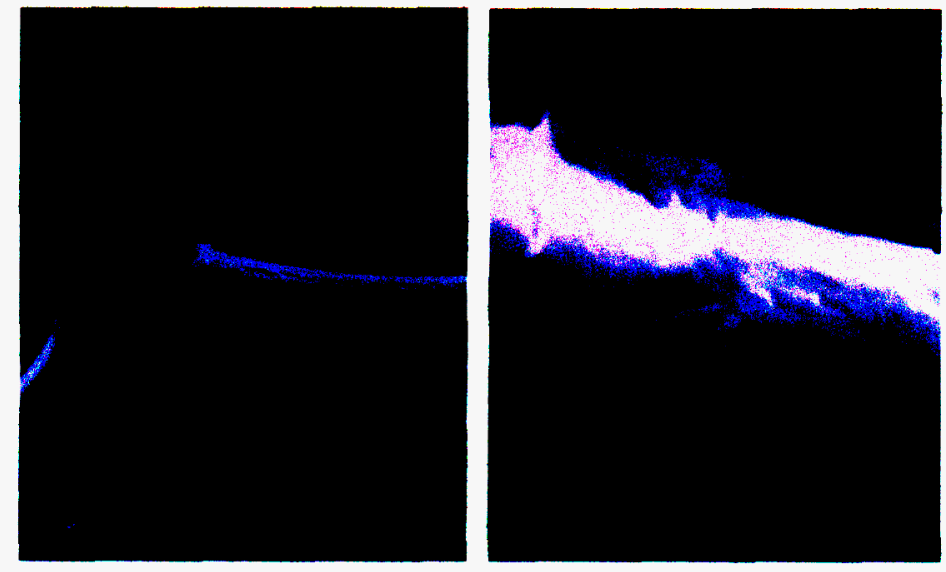

Figure 5: $\sim 15 \mu \mathrm{m}$ thick $\mathrm{CH}-\mathrm{shell}$ wall and $\sim 10 \mu \mathrm{m}$ thick convectively baked $\mathrm{P}$ coating at $400 X$ and $2400 X$. 
The following figure is another IR soft-baked sample comprising of 5 IR cycles for a total of $\mathbf{2 4}$ wet coats. Although there is a puckered region on the left-handside, the center region indicates a smooth surface. Samples shown in Figures 4 and 6 indicate that IR curing requires further experiments to understand how IR interacts with the PA layers and the underlying $\mathrm{CH}$ layer, such that we can produce smooth, cured P layers within NIF specifications.

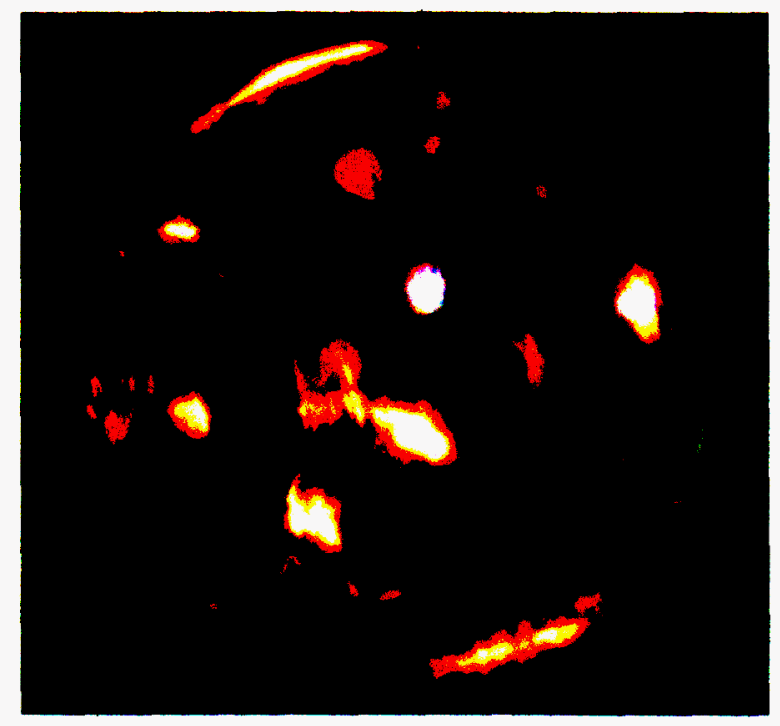

Figure 6: 24 coat PA sample consisting of 5 IR cycles.

A summary of agreed activities to be executed for the remaining portion of Task $A$ are as follows:

1. Optimize, automate and demonstrate repeatable procedures to deposit wide coating bands with thickness of $>50 \mu \mathrm{m}$ on stalk-mounted shells such that thickness uniformities are $<1 \mu \mathrm{m}$.

2. Conduct further tests and refine reliable recipes to incorporate wavelength specific IR radiation and emitter geometry to soft-bake and possibly imidize P-coated $\mathrm{CH}$-shells.

3. In completing Step 1 and 2, demonstrate a clear plan on methods proposed to transition to acoustically levitated shells and maintain surface requirements.

4. Perform in-house evaluation and develop methods to address suitability of an alternative, all-ceramic ink-jet dispenser manufactured by InkJet Technology, Inc. 
5. Provide LLNL with soft-baked and imidized P-coated $\mathrm{CH}$-shell samples for surface analysis. 


\section{Sixth Progress Report:}

\section{NIF Polyimide Ablators Produced by Solution Spray Techniques}

Since the last review, we have a fully operational and automated system for uniformly coating and curing vacuum-mounted $\mathrm{CH}$-shells with ink-jetted PA (polyamic acid). Our custom motion-control software continuously translates a rotating stalk-mounted shell past the inkjet while varying the shell's rotation rate. This coated shell is then moved to a softbake position (as shown in figure 1) and the cycle is then repeated for further depositions. All deposition and softbake parameters are software modifiable. With the assistance of Luxel's machine shop, we have developed a rotating vacuumchuck assembly that holds shells at low ( 7 PSIA) pressure throughout the coating process. This has eliminated stalk-mounting epoxies that interact with NMP, the time required to mount shells, and greatly improved radial runnout $(<10 \%$ of shell diameter). These enhancements significantly improve the continuity of ink-jetted liquid layers. We are currently able to attain a coating rate of $\sim 5 \mu \mathrm{m} /$ hour of softbaked $P$ (polyimide) when using our new, focused IR lamp (Spot IR emitter). Softbake times are currently 35 to 55 seconds dependent upon total coated thickness.

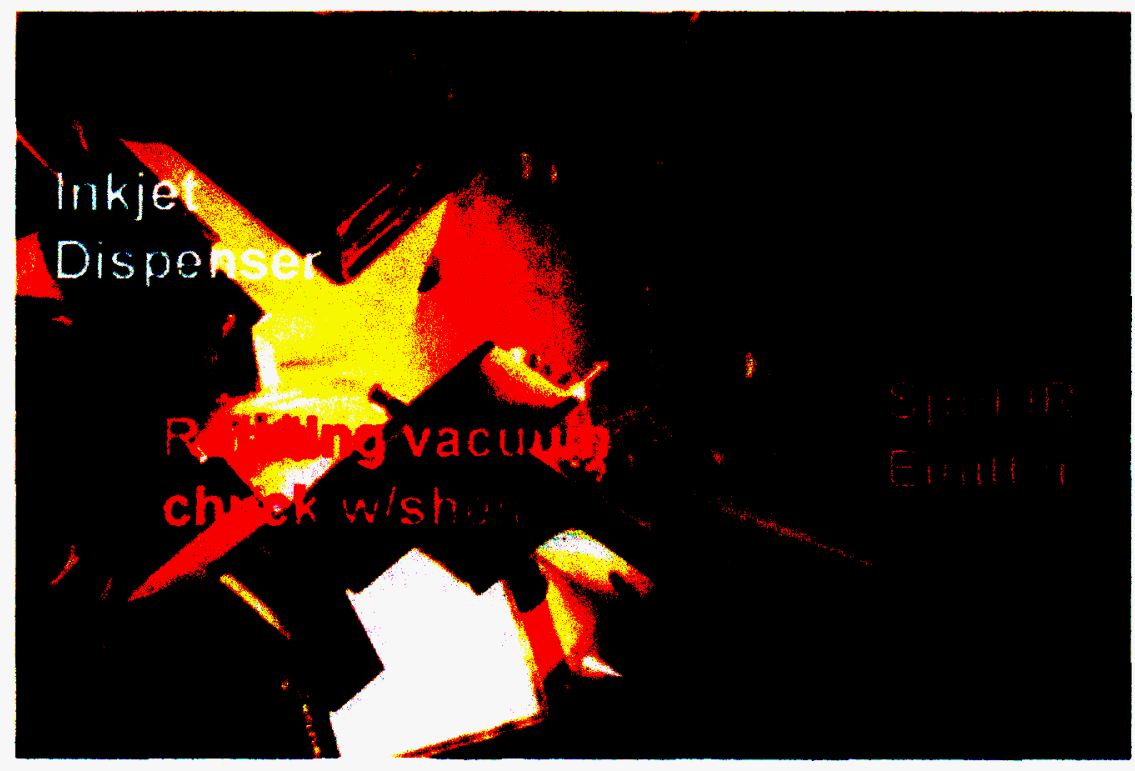

Figure 1: Current Equipment Setup

Most recently, we have focused on establishing a coat/cure-cycle that produces visually-smooth softbaked samples. We have succeeded in this task by depositing single wet coats per each coating event and using wavelength-specific, focused IR, producing short softbake times. Based on black-body curves of our focused IR-lamp as a function of filament-temperature, we have optimized the voltage output to the lamp such that its peak power is centered at $\lambda \sim 3.5 \mu \mathrm{m}$ (figure 2). 


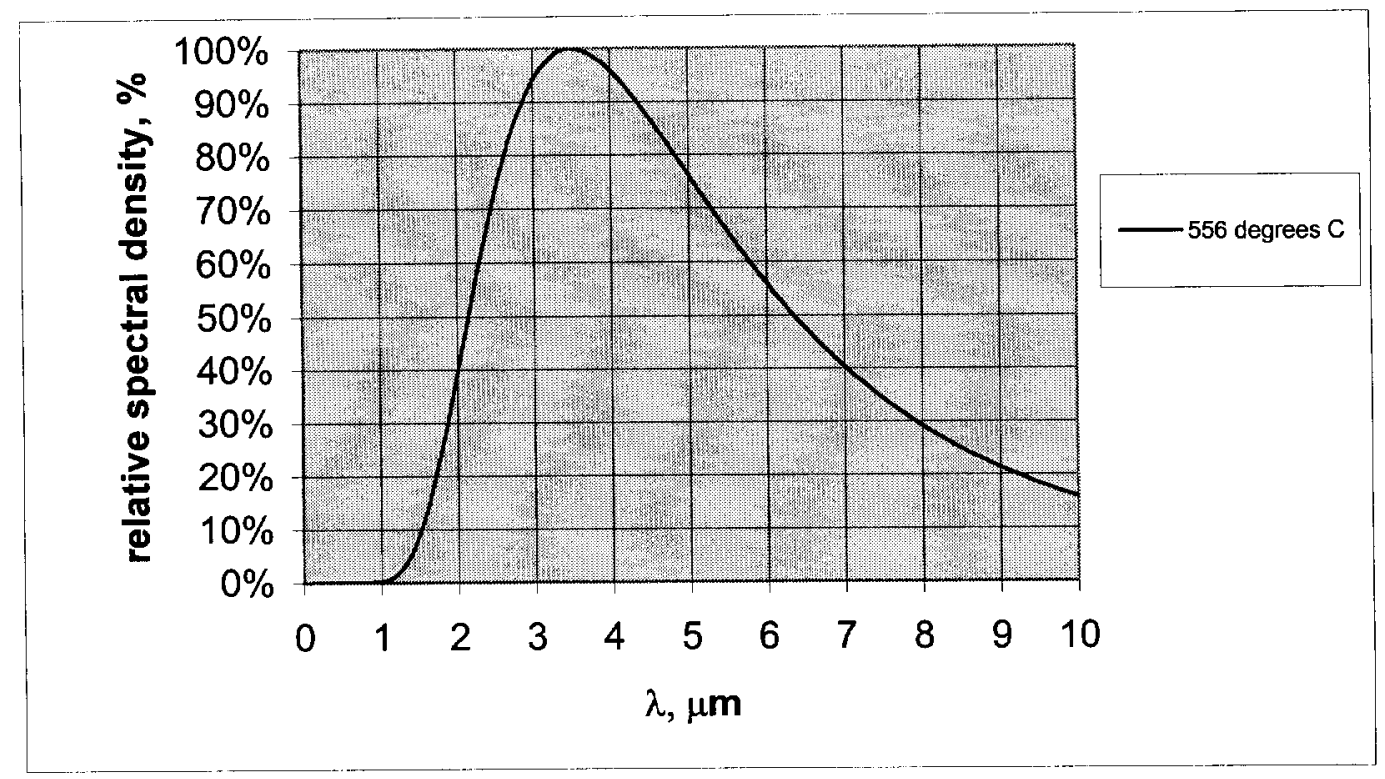

Figure 2

At full operating voltage, the filament-temperature is $2227^{\circ} \mathrm{C}$. This produces a short IR spectrum that peaks at $\lambda \sim 1.2 \mu \mathrm{m}$. Reducing this voltage by $\sim 25 \%$, the filament-temperature reduces to $556 \mathrm{deg} C$, producing the spectral response shown in figure 2, which is the ideal spectral range for curing $P A->P$. Additionally, a quartz face-shield is used to filter-out wavelengths beyond $4 \mu \mathrm{m}$.

The IR-lamp has a power density at its focal point formed by the ellipsoid reflector of 1.7 watts $\mathrm{mm}^{-2}$ at full power (i.e., full operating voltage). The focal point is $2.8 \mathrm{~cm}$ from the lamp source and has a diameter of $6 \mathrm{~mm}$. Placing a $\mathrm{CH}$ shell $2.8 \mathrm{~cm}$ from this focal point is twice as far from the lamp source. Assuming that the power density is one-fourth of that at the focal point, the power density at the shell is 0.4 watts $\mathrm{mm}^{-2}$. This power is distributed over the spectrum as shown in figure 3 (this and other spectrums were calculated using on-line calculators supplied by our IR-lamp manufacturer, www.researchinc.com).

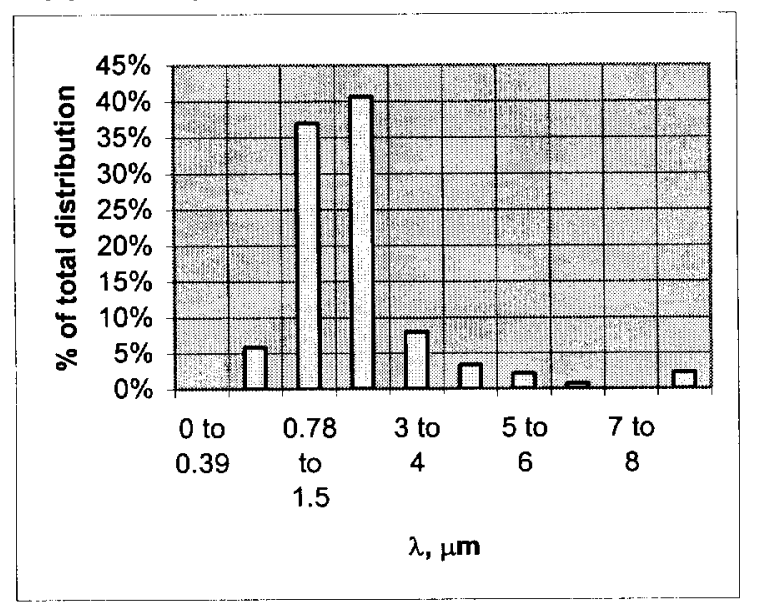

Figure 3

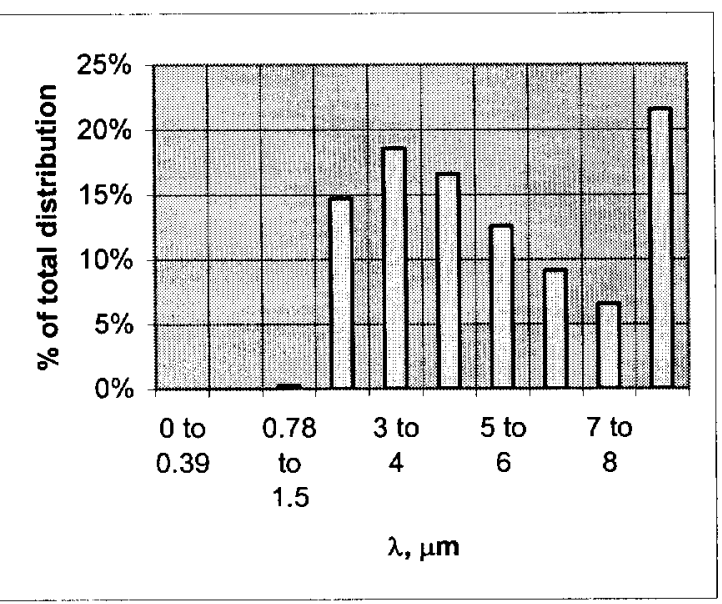

Figure 4 
Reducing the filament temperature to $556^{\circ} \mathrm{C}$ results in the shifted power distribution shown in figure 4 . The spectral range effective in curing the $P A->P$ is in the 3 to $4 \mu \mathrm{m}$ region is also shown in figure 4 . This power density is $\sim 19 \%$ of the total emissive power at $556^{\circ} \mathrm{C}$

The total emissive power of an ideal blackbody emitter at a given temperature, $T$, is given by the Stefan-Boltzman law, $E_{b}=\sigma T^{4}$, where $\sigma$ is the Stefan-Boltzman constant having a value of 0.0567 watts $\mathrm{mm}^{-2} \mathrm{~K}^{-4}$ and $\mathrm{T}$ is in Kelvin. For a real emitter having constant emissivity, $\varepsilon$, its total emissive power is $E=\varepsilon E_{b}=\varepsilon \sigma T^{4}$. Therefore, emissivity of our IR-lamp is $\varepsilon=E / E_{b}$. E for our IR-lamp at full power $(T=2500 \mathrm{~K})$ is published to be 1.7 watts $\mathrm{mm}^{-2}$. Therefore, $\varepsilon \sim 1.7 / 2.2 \sim 0.8$ and $E(T=829 \mathrm{~K}) \sim 0.8^{\star} 0.03$ watts $\mathrm{mm}^{-2} \sim 0.02$ watts $\mathrm{mm}^{-2}$.

Using the above assumption that the power density decreases four times when the shell is twice the focal distance from the source, the total power density on the shell is $E(T=829 \mathrm{~K}) / 4 \sim 6$ milliwatts $\mathrm{mm}^{-2}$. Given that the shell's crosssectional area is $\sim \pi \mathrm{mm}^{2}$, the total incident power is $\sim 20$ milliwatts. Finally, the power inicident on the shell between 3 and $4 \mu \mathrm{m}$ is $\sim 4$ milliwatts.

The total inicident power, $p$, heat capacity, $c$, of NMP, and total liquid mass of PA/NMP can be used to estimate the time required to raise the temperature of a liquid coated shell from ambient to some final temperature. We have measured this final temperature to be $\sim 60^{\circ} \mathrm{C}$ at twice the focal distance. $\mathrm{c}$ of NMP varies $<14 \%$ between 25 and $100^{\circ} \mathrm{C}$ and $\mathrm{c}\left(\mathrm{T} \sim 50^{\circ} \mathrm{C}\right)=1.95 \mathrm{~J} \mathrm{~g}^{-1}{ }^{\circ} \mathrm{C}$. Liquid mass of a single coat of $3 \% \mathrm{PA} / 97 \% \mathrm{NMP}$ is measured to be $\mathrm{m} \sim 5 \mathrm{e}-4 \mathrm{~g}$. The time to raise the temperature from $T_{1}=25$ to $T_{2}=60^{\circ} \mathrm{C}$ is:

$$
\text { Time }=\left(T_{2}-T_{1}\right) c \mathrm{~m} \mathrm{p}^{-1}=6 \text { seconds }
$$

We find the rate of curing PA to $P$ is found to explicitly control the degree of surface roughness. We are currently optimizing this rate through experimentation to improve surface finish of P-coated shells. 


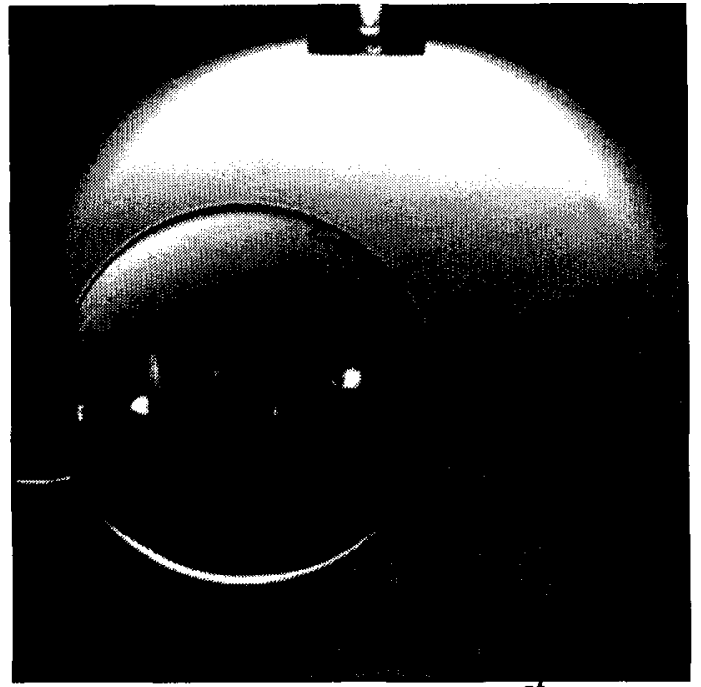

Figure 5: Coated shell after $1^{\text {st }}$ softbake

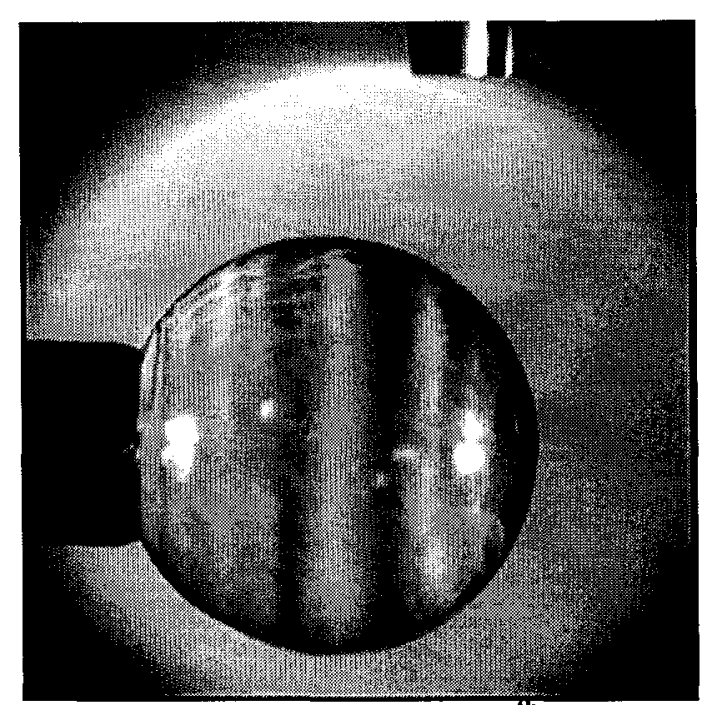

Figure 6: Coated shell after $10^{\text {th }}$ softbake

After numerous experimental trials, we have refined our coating/bake parameters to produce acceptable shells using single wet coats and focused IR heating. The shell shown in Figures 5 and 6 are in-situ photomicrographs from our latest sample sent to LLNL for characterization. The shell, identified as AlphaTango, has ten single coats, where each coat was followed by a softbake averaging $\sim 37$ seconds. The first coat upon the $\mathrm{O}_{2}$-etched shell surface wets out and bakes superbly as seen in Figure 5, whereas each successive coat's wet-out is strongly dependent upon the amount of softbake time from the previous coat. If the softbake-duration's ramp-rate increase is too slow, unevaporated NMP remains and begins building up (liquid) thickness in subsequent coating-baking cycles. This eventually leads to shell collapse. If this ramp-rate increase is too fast, subsequent drops do not wet out well (compared with PA wetting on a bare, Oetched shells), leading to a visually rough-looking surface.

This behavior was recently discovered (using our in-situ imaging system) as we have moved to depositing a single wet coat per bake cycle rather than multiple wet coats as previously experimented. We are currently experiencing variations in wetability between coats with as-little-as 5 second changes in softbake duration. Our current process increases the softbake-duration by 3 to 5 seconds per each successive coat, after an initial softbake-duration of $\sim 35$ seconds.

We previously assumed heating times would have to be decreased as thickness and volume increased, but experiments indicate that softbake times required to drive off NMP increase as the shell gains mass and volume. Furthermore, at $\sim$ coat \# ten, we observe that this required time begins to decrease to as low 10 seconds! This indicates the emissivity of coated shell may change significantly with coating-thickness. Further experiments will be needed to understand these changing material properties, enabling us to reliably produce NIF quality shells. 
The use of a vacuum may assist the NMP solvent evaporation process by lowering the ambient pressure relative to the NMP vapor pressure. This could reduce the temperature and time required for soft baking and minimize the nonlinear process of emissivity changes as coating layers are added. IR would then be used with appropriate ramp-ups for imidization.

Another possible advantage of a vacuum is reducing the ink-jet drive energy required to expel drops, thereby prolonging the jet's life. With a transparent vacuum chamber, equipment such as cameras could be placed outside the chamber.

A summary of activities to be executed for the remaining portion of Task $A$ are as follows:

1. Investigate and understand IR softbake cure times, and changing emissivity as a function of thickness to develop a process to produce thick, smooth coatings.

2. Optimize and demonstrate repeatable procedures to deposit wide coating bands with thickness of $>50 \mu \mathrm{m}$ on stalk-mounted shells such that thickness uniformities are $<1 \mu \mathrm{m}$. If deemed necessary, upgrade our existing linear stages and stepper motor controllers to enable 1 to $10 \mu \mathrm{m}$ of positioning precision.

3. Provide LLNL with softbaked and imidized P-coated $\mathrm{CH}$-shell samples for surface and thickness characterizations.

4. Conduct tests and define reliable methods to further incorporate wavelength specific IR radiation, emitter geometry, and changing emissivity to softbake and possibly imidize $\mathrm{P}$-coated $\mathrm{CH}$-shells.

5. In completing Steps 1 through 4, demonstrate a clear plan on methods proposed to transition to acoustically levitated shells and maintain geometry and surface requirements.

6. Perform in-house evaluation and develop methods to address suitability of alternative ink-jet dispensers from InkJet Technology, Inc. and Engineering Arts. 


\section{urit \\ LUXEL CORPORATION}

Solution-based polyimide deposition process development - Final Repurt

In the solution based approach the plan is to deposit droplets of polyimide precursor solution (polyamic acid plus the solvent NMP) onto acoustically levitated mandrels to form uniform fluid layers that are partially cured (soft baked) to remove the solvent before the next layer is applied. After the required thickness is obtained, the polyimide shell would then be imidized at an elevated temperature to complete the process and realize the full strength of the material.

The solution based approach produces polyimide of very high strength (over $300 \mathrm{M} \mathrm{Pa}$ ) with an inherently smooth surface due to a strong surface tension effect present when the polyamic acid solution is in liquid form. By spraying a mandrel in levitation, it may be possible to produce high quality target capsules that are defect free, partly because they are never touched by man or machine. This approach is founded on ten years of funded research from NASA where techniques for fabricating polyimide films of high strength, especially at cryogenic temperatures, have been developed.

The work described here has focused on the deposition and cure steps by coating mandrels that are mounted on stalks in order to develop processes that are adaptable to levitation. It is planned that during FY 2003 the transition to levitated mandrels will be accomplished.

Considerable effort has been directed at getting a uniform smooth coating using an atomizer as the spray source. Specifically, a series of experiments indicated that transition to a smooth, $\sim 100 \AA$ RMS surface occurs at a liquid thickness of $\sim 4000 \AA$. Using this as a guideline, the work was focused on demonstrating the ability to apply multiple coatings on a spherical mandrel. In the course of this effort, it was determined that oxygen etching the mandrel before the first coat is applied helped to initial coat to wet properly. Since then it has been standard practice to oxygen etch all mandrels used by Luxel in the spray coating process.

Then attention was directed at achieving good control of the soft baking process as is required to drive off the solvent. In these experiments, a stalk-mounted mandrel was used. Feedback controlled convective heating was implemented. An effective ramp and dwell temperature cycle was developed and the process was automated to run unattended. Multiple coat samples were produced with coatings as thick as $70 \mu \mathrm{m}$. This was accomplished by applying individual coats with a thickness of between 0.5 and $1.0 \mu \mathrm{m}$ after soft baking.

LLNL WYKO characterization of the $70 \mu \mathrm{m}$ thick sample (before imidization) showed it to be very smooth locally (Figure 1), radiography showed the coatings to be very uniform with no evidence of the individual layers or delamination at the mandrel interface (Figure 2), and the radiography/shadowgraphy shows the walls to be $70 \mu \mathrm{m}$ thick and uniform to within a few microns (Figure 2). Clearly the surface tension smoothing effect seen previously can be used to aid in fabricating a multilayer coating. However, as expected, some lower mode asymmetry was evident

515 Tucker Ave. Friday Harbor, WA 98250 USA
Tel: (360) 378-4137 Fax: (360) 378-4266 email: luxel@luxel.com 
and this requires further effort. Subsequent imidization of the sample at $300^{\circ} \mathrm{C}$ resulted in no significant geometric or morphological changes.

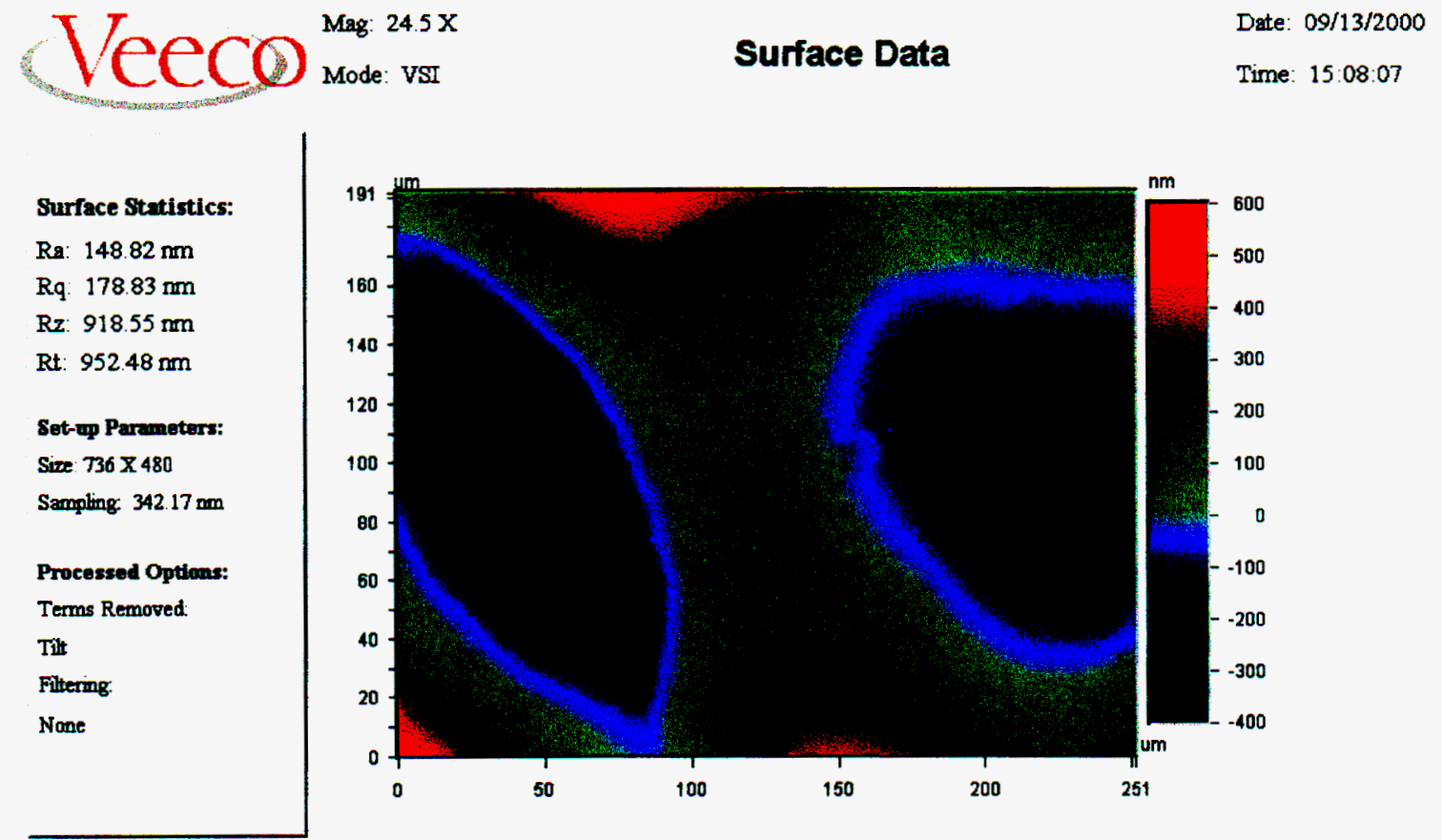

Title: Luxel 50 coat View 3

Note: 25X Sprayed softbake PI coated CH shell

Figure 1. WYKO interferometric image of 250 by $190 \mu \mathrm{m}$ patch on the $70 \mu \mathrm{m}$-thick coating. Note surface is locally smooth, but thickness variations of about $1 \mu \mathrm{m}$ are present over distances of about $100 \mu \mathrm{m}$.

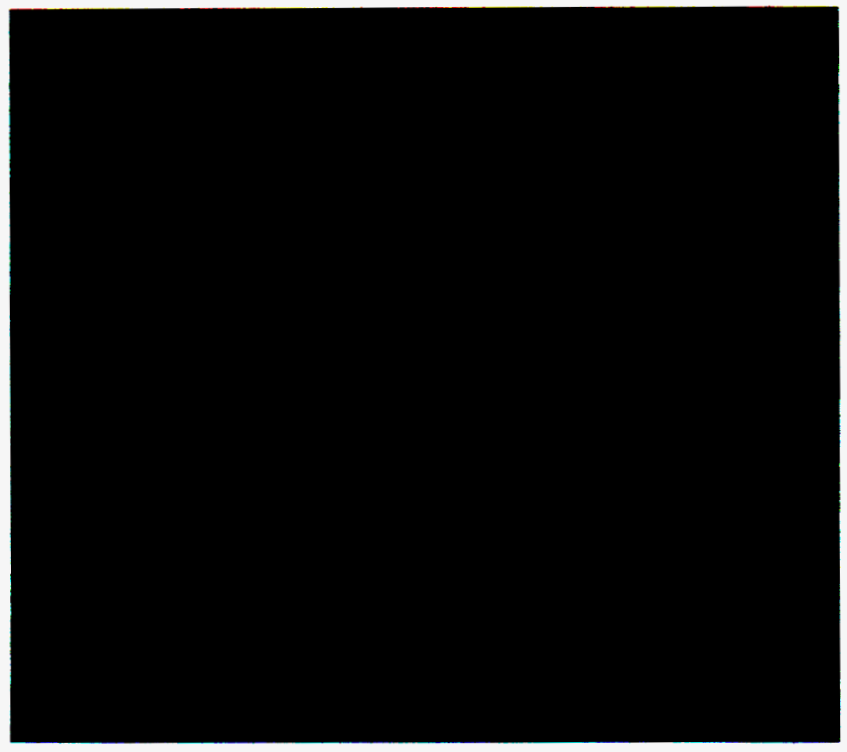

515 Tucker Ave.

Friday Harbor, WA 98250 USA
Tel: (360) 378-4137 Fax: (360) 378-4266 email: luxel@luxel.com 
Figure 2. Radiograph of a portion of the $70-\mu \mathrm{m}$ thick coating. The bright line marks the inner wall, the fainter line near it the outside of the initial $\mathrm{CH}$ mandrel. Note the coating appears very uniform and there is no evidence of delamination at the mandrel/coating interface. Radiographs of the imidized coatings are unchanged.

Even though these results were encouraging, it was recognized that an atomizer might not be the best device for delivering the spray in this application. The atomizer produces a spray that contains droplets with diameters varying from 1 to $100 \mu \mathrm{m}$ with a bell curve distribution and hence is difficult to control to the extent needed. Replacement of the atomization-based delivery system with an inkjet system capable of precise placement of individual micro-drops ( $\sim 50 \mu \mathrm{m}$ in diameter) will provide much greater control over the process.

In anticipation of the need to improve spray process control, Luxel investigated the use of ink-jet spray technology. Several different ink-jets that are compatible with the polyamic acid solution were found. The advantage of this approach is that drop-on-demand capability under computer control can be used to precisely place the spray solution droplets exactly where they are needed. After considerable experimentation, this was shown to be possible by precisely laying down a continuous overlapping spiral pattern of drops on a rotating stalk mounded mandrel. Again the process is fully automated to take advantage of the surface tension effect to produce a uniformly smooth, liquid coating over the area of the mandrel that was covered by the droplets (Figure 3 ).

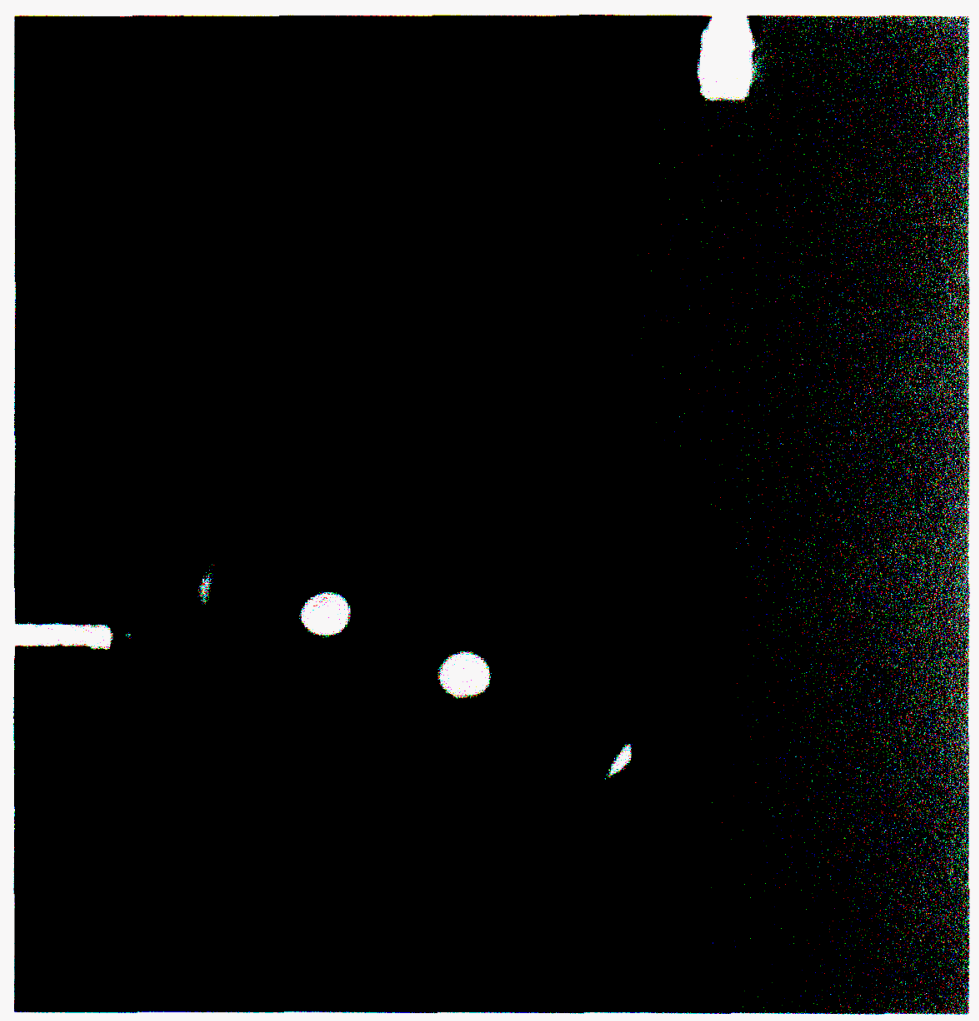

Figure 3. A mandrel after 9 coating cycles, Infrared cured, each bake cycle $=7 \mathrm{~min}$ total time @210C, and final imidize ramp bake=10min total @325C

515 Tucker Ave.

Friday Harbor, WA 98250 USA
Tel: (360) 378-4137 Fax: (360) 378-4266 email: luxel@luxel.com 
An indication of the smoothness of the coatings is shown by the uniformity of the highlights on the sphere that come from the illuminating lights. Granted, the coating is less than $10 \mu \mathrm{m}$ thick but the visual smoothness evident in the photograph is an indication of what may be possible. This was accomplished in spite of all the limitations and irregularities associated with the stalk-mounted mandrel. It is not unlike the situation with DT ice in that if and when the right conditions exist, a material with remarkable thickness uniformity and smoothness can be created. The potential of dropon-demand ink-jet technology has been demonstrated for this application.

Also, in an attempt to speed up the soft baking process, the heating was changed from convective heating to IR heating. The IR heating was accomplished using lamps known to provide radiation in the region where the polyamic acid is highly absorptive. It was found that with IR heating, it is possible to soft bake the spray-coated layers in seconds rather than minutes. However, after a coating with a total thickness of about $10 \mu \mathrm{m}$ was accumulated, the microsphere began to become distorted. Apparently, IR heating causes undesired side effects that need to be better understood. Further effort has shown that convective heating does not cause this problem.

Significant progress has been made is showing that a spray based approach has the potential for providing a method of producing high quality target capsules for the NIF and thus additional effort to develop this approach is warranted. The move to acoustic levitation is planned in the near future.

The dual-horn, single-axis levitator leased from CRI Inc. shows some promise as a means of levitating shells during inkjet coatings and curing of PAA. It is able to levitate shells that acquire $\sim 1$ mg of PAA during inkjet deposition which are then subject to IR and convective curing methods lasting minutes to $\sim 1$ hour, respectively. Coated shells have been observed to levitate for $\sim 3$ days unattended.

Modifying the intensity and relative phase of the two horns reasonably controls a bare shell's rotation rate. Its orientation is not so well controlled. When a bare shell's translation and angular velocities are $\sim 0$ and its axis-of-rotation is parallel to gravity, depositing inkjet droplets (diameter $\sim 70 \mu \mathrm{m}$, drop-frequency $10 \mathrm{~Hz}$ ) near its top pole causes this pole to slowly "tip-over" toward the bottom as liquid mass grows locally. Under these conditions, we have observed a nearly complete coating of a bare shell in $\sim 4$ minutes. Due to the form of a single-axis, acoustic-field form, PAA is thicker at equator and thinner at the poles-see figure 4 . When velocity conditions are not zero, the axis of rotation can changes randomly on $\sim 0.1 \mathrm{sec}$ time-scale. Under this condition, bare-shells have been observed to be coated in a similar period of time.

515 Tucker Ave.

Friday Harbor, WA 98250 USA
Tel: (360) 378-4137 Fax: (360) 378-4266 email: luxel@luxel.com 


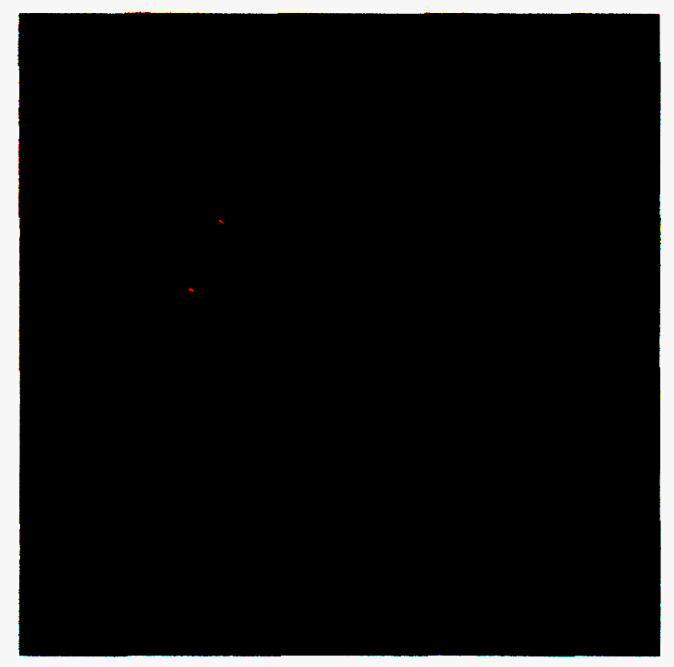

Figure 4

A benefit of the current levitator over the original single-horn one, is its accommodation of large objects in the acoustic field while levitating coated shells-see figure 5 . This has enabled us to introduce Luxel's Radak I and II vacuum furnaces into the field, near a shell, for convective softbaking. During a convective cure cycle shell stability degrades, due to reduced acoustic field strength and increasing convective-flow forces. Reduction in field strength results from disrupting the acoustic field with the furnace and the inkjet (particularly its housing). We are in the process of optimizing a coil heater, inkjet housing, and their placements to minimize this disruption. As part of automating and optimize our levitation-deposition system, we intend to remove the furnace during deposition and the inkjet during curing. This will improve the stability and potentially the control of levitated shells.

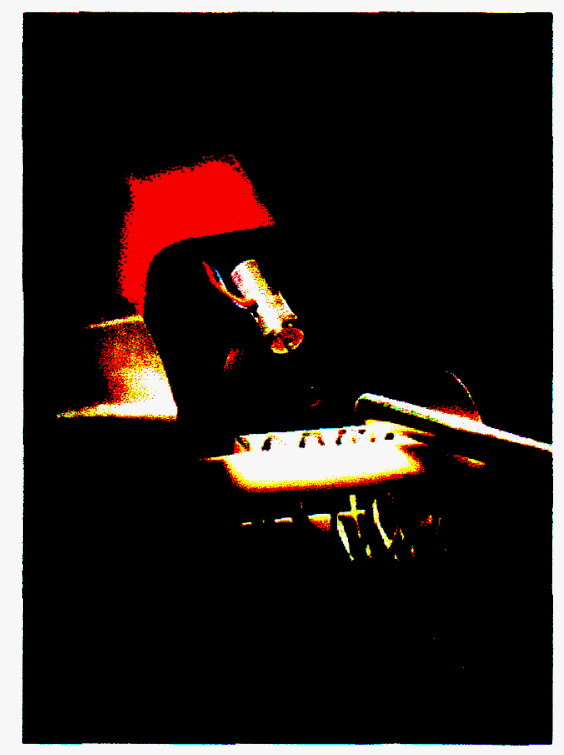

Figure 5

515 Tucker Ave. Friday Harbor, WA 98250 
As levitated shells are coated they occasionally oscillate parallel to gravity, with peak-to-peak amplitudes reaching a shell diameter. The frequency of these oscillations must be $>15 \mathrm{~Hz}$ because shells appears as extended, cylindrical blurs when viewed with a digital far-field microscope having a 15 frames/sec throughput. After a low-temperature softbake $\left(<70^{\circ} \mathrm{C}\right)$, we are unable to sharply focus on the edge of a shell, due to low-amplitude (of-order $10 \mu \mathrm{m}$ ), high frequency $(>15 \mathrm{~Hz}$ ) oscillations. However, by modifying the relative phase of the two horns, and minimizing convectively-driven forces (e.g. bright lighting) and mechanically-driven air-flow forces (e.g. equipment fans), we able to greatly stabilize a levitated shell. In particular, we are able to regain $\sim 0$ translation and angular velocities. It remains to be seen if we can obtain this stability throughout a convective or IR cure cycle.

Given the operating sound level of the levitator is $\beta \sim 150 \mathrm{~dB}$ (quoted by CRI Inc.), the average power, $P_{\text {avg, }}$ incident on a shell may be estimated as follows (Physics for Scientist and Engineers, Serway, 2000, $5^{\text {th }}$ edition). $\mathrm{P}_{\text {avg }}$ is found through the relation, $I=\frac{P_{\text {avg }}}{A}$, where $\mathrm{I}$ is the intensity of the sound waves in units of power per unit area. I is obtained from the definition $\beta \equiv 10 \log \left(\frac{I}{I_{0}}\right)$, where $\mathrm{I}_{0}$ is the threshold of human hearing defined to be $\mathrm{I}_{0} \equiv 10^{-12} \mathrm{~W} / \mathrm{m}^{2}$ at $1 \mathrm{KHz}$. Using the surface area of a $2 \mathrm{~mm}$ radius shell for $\mathrm{A}, \mathrm{P}_{\mathrm{avg}} \sim 10 \mathrm{~mW}$. This is comparable to the incident power of our focused IR heater that has been estimated (in a previous progress report) to be $\sim 4 \mathrm{~mW}$ between 3 and $4 \mu \mathrm{m}$. This indicates the possibility that the NMP evaporation rate is increased and some degree of imidization is occurring due to sound-wave energy.

Figure 6 is a back-lit image of a levitated shell that received 9 coats from an inkjet. After each coat, a low temperature $\left(<70^{\circ} \mathrm{C}\right)$ cure was performed, lasting 1 hour. The evident roughness results from significant levitation instabilities described above. The inkjet is also a source of roughness due to its stream breaking up near its tip during levitation. This condition required the inkjet tip to be $\sim 2 \mathrm{~mm}$ from the shell, further adding to its instability. A shadowgraph-derived PSD for modes $<60$ is shown for this shell in Figure 9-red line.

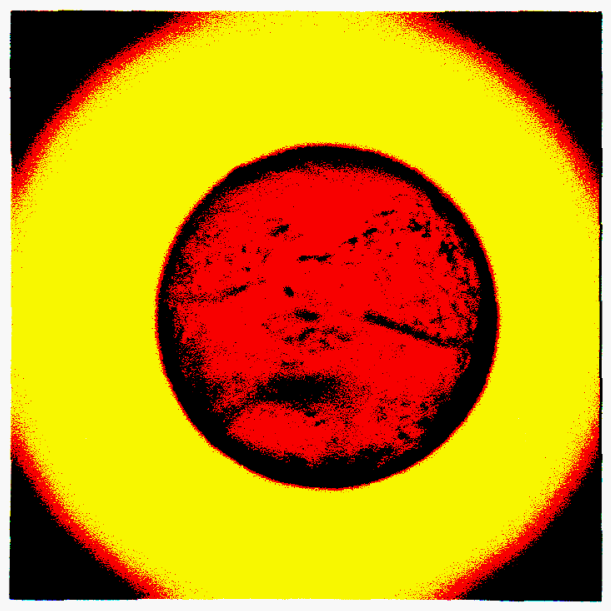

Figure 6

515 Tucker Ave.

Friday Harbor, WA 98250 USA
Tel: (360) 378-4137 Fax: (360) 378-4266 email: luxel@luxel.com 
Following on the above observation that sound wave energy may evaporate NMP, we immersed a $\mathrm{CH}$-shell in PAA and placed it in levitation. The shell acquired $\sim 2 \mathrm{mg}$ of PAA. During $\sim 1$ hour it evaporated. This is indicated in the series of images shown in Figure 7-time increases from left to right. The dry-liquid interface is a horizontal line with the dry area appearing dimmer than the bright liquid area-gravity points toward the bottom of the images
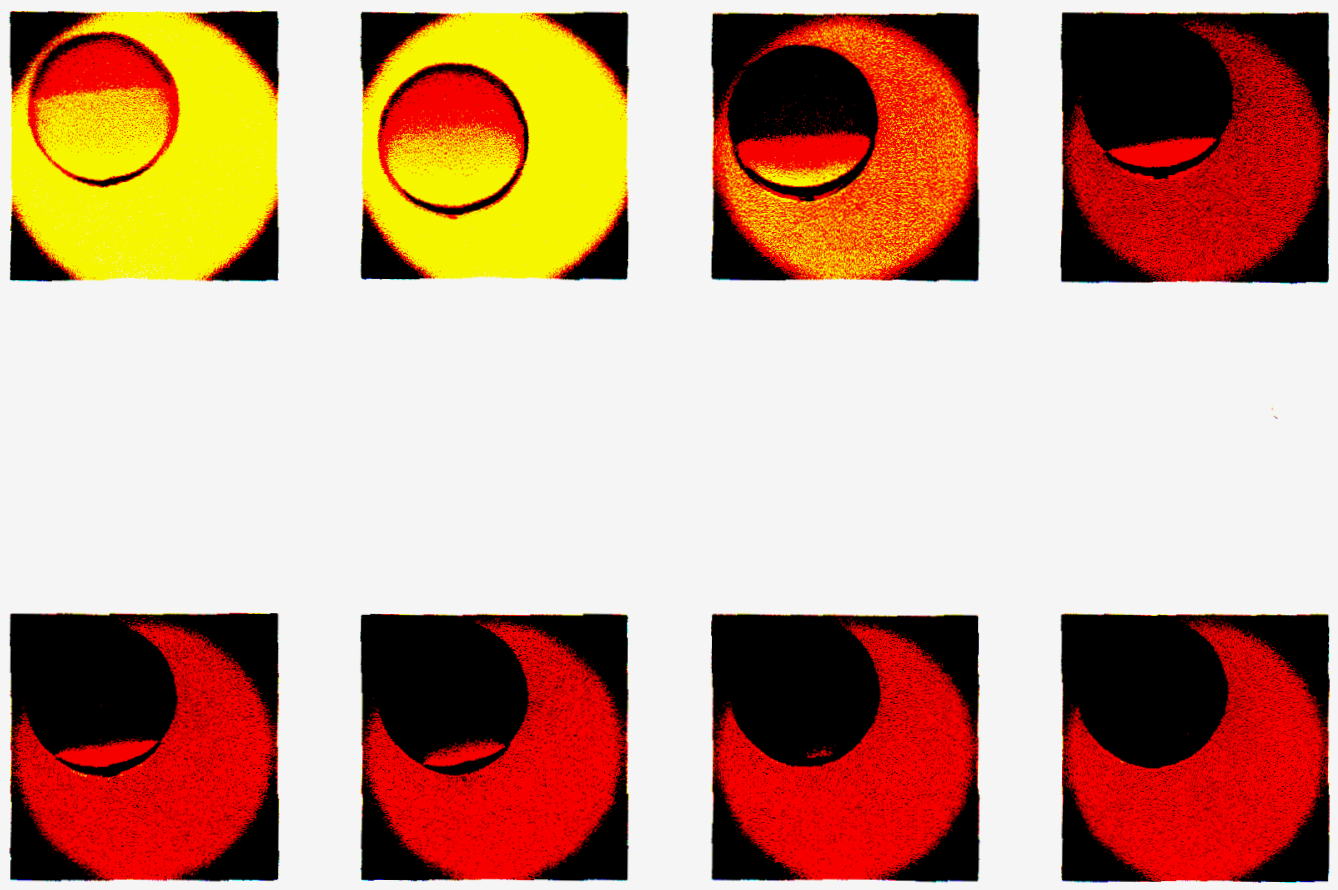

Figure 7

Stability of the shell during this period was dramatically improved by de-phasing the two acoustic fields and reducing the power to point that minimally supported the shell. Figure $8 \mathrm{a}$ is shadowphotomicrograph of this shell after 6 coats-noticeable defects are due to accidental shell-droppings. Figure $8 \mathrm{~b}$ is the same shell under different lighting. The PSD of figure $8 \mathrm{a}$ shown in Figure $9-$ blue line.

515 Tucker Ave.

Friday Harbor, WA 98250 USA
Tel: (360) 378-4137 Fax: (360) 378-4266

unw.luxel.com

email: luxel@luxel.com 

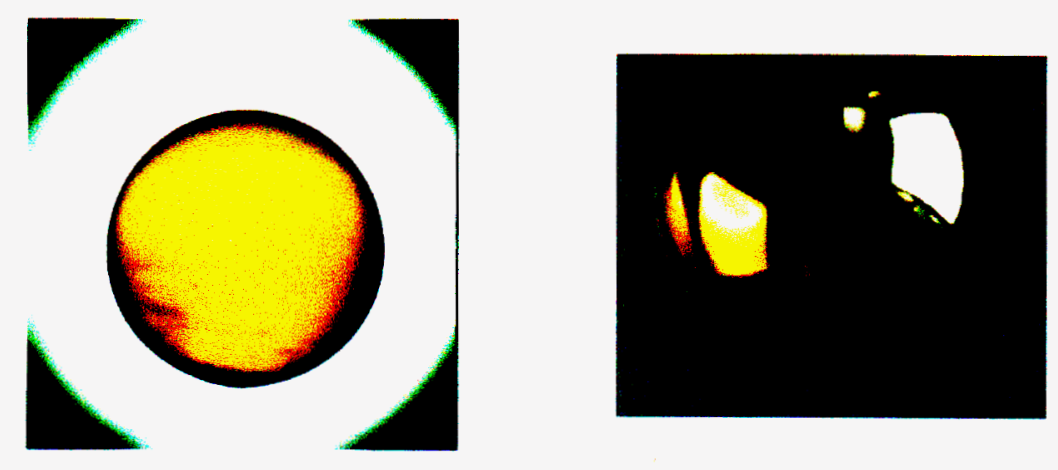

Figure 8: a-left and b-right

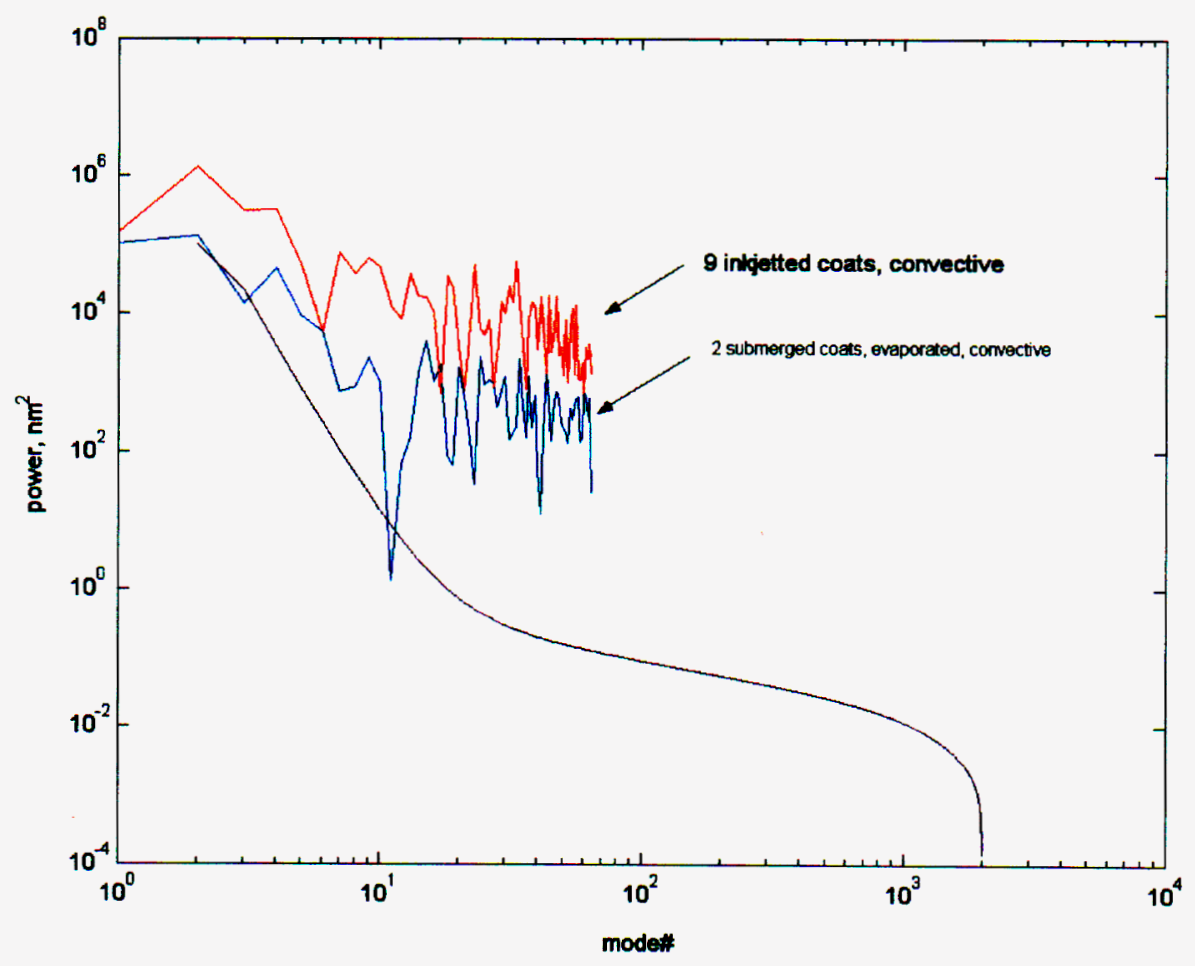

Figure 9

515 Tucker Ave.

Friday Harbor, WA 98250 USA
Tel: (360) 378-4137 Fax: (360) 378-4266

unw.luxel.com email: luxel@luxel.com 
In summarizing our effort over the past 2 years, Tables 1 and 2 indicate the advantages and disadvantages inherent in the PAA delivery systems, cure methods, and handling approaches explored.

ICF POL YIMIDE TARGET

FABRICATION PROCESS COMPARISONS Advantages (+) / Disadvantages (-)

\begin{tabular}{|c|c|c|c|}
\hline & STALK MOUNTED & $\begin{array}{c}\text { AERO ACOUSTIC } \\
\text { LEVITATION }\end{array}$ \\
\hline \multirow{3}{*}{ 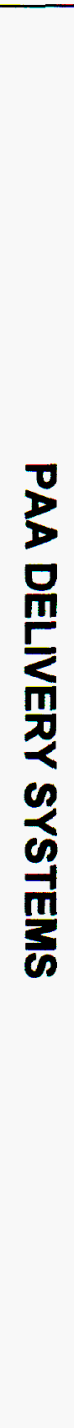 } & $\begin{array}{l}\text { Drop on- } \\
\text { Demand } \\
\text { InkJet }\end{array}$ & $\begin{array}{l}\text { + Drop-on-demand } \\
\text { placement of discreet or } \\
\text { continuous drops, precise } \\
\text { mass control, } 84 \text { um coating } \\
\text { thickness has been } \\
\text { achieved. Has run for } \\
\text { several unattended days. } \\
\text { - Delicate system requiring } \\
\text { extensive operator input. } \\
\text { Low viscosity, hence low } \\
\text { PAA concentration required. }\end{array}$ & $\begin{array}{l}\text { + Potential for precise placement and } \\
\text { control of droplets with increased operating } \\
\text { voltage. } \\
\text { - Loss of drop placement accuracy, stream } \\
\text { breakup, and close proximity of inkjet } \\
\text { degrades shell stability resulting in coat } \\
\text { roughing. }\end{array}$ \\
\hline & $\begin{array}{l}\text { Ultrasonic } \\
\text { Atomizer }\end{array}$ & $\begin{array}{l}\text { + Able to provide } 1 \text { to } 100 \\
\text { um diameter droplets upon } \\
\text { CH mandrel, } 70 \mu \mathrm{m} \text { coating } \\
\text { thickness has been } \\
\text { achieved. Higher } \\
\text { concentration PAA } \\
\text { permittable. } \\
\text { - Device occasionally 'spits' } \\
\text { large, }>100 \mu \mathrm{m} \text { droplets. }\end{array}$ & $\begin{array}{l}\text { + Same as stalk; cloud of atomized PAA } \\
\text { completely coats non-etched and etched } \\
\text { shells. Higher concentration PAA } \\
\text { permittable. } \\
\text { - Large droplets can eject levitated shell } \\
\text { and proximity of atomizer may degrade } \\
\text { shell stability. }\end{array}$ \\
\hline & $\begin{array}{l}\text { Bath } \\
\text { Immersion }\end{array}$ & $\begin{array}{l}\text { + Permits rapid and } \\
\text { complete coverage of shell } \\
\text { - stalk disrupts uniform liquid } \\
\text { coverage about the shell. }\end{array}$ & $\begin{array}{l}\text { + Permits rapid and complete coating of } \\
\text { shell. Highest concentration PAA } \\
\text { permittable. Etched shells may not be } \\
\text { required. Enhanced NMP evaporation ( } 1 \\
\mu g /\left(\text { sec } \mathrm{mm}^{2}\right) \text { ) removes majority of solvent } \\
\text { without conventional softbaking. Using } \\
\text { suitable handling, contribution to surface } \\
\text { roughness is visually undetectable. } \\
\text { - Handling is difficult, slumping may be } \\
\text { present on lower hemisphere }\end{array}$ \\
\hline
\end{tabular}

Table 1

515 Tucker Ave.

Friday Harbor, WA 98250 USA
Tel: (360) 378-4137 Fax: (360) 378-4266 email: luxel@luxel.com 
ICF POL YIMIDE TARGET

FABRICATION PROCESS COMPARISONS

Advantages (+) / Disadvantages (-)

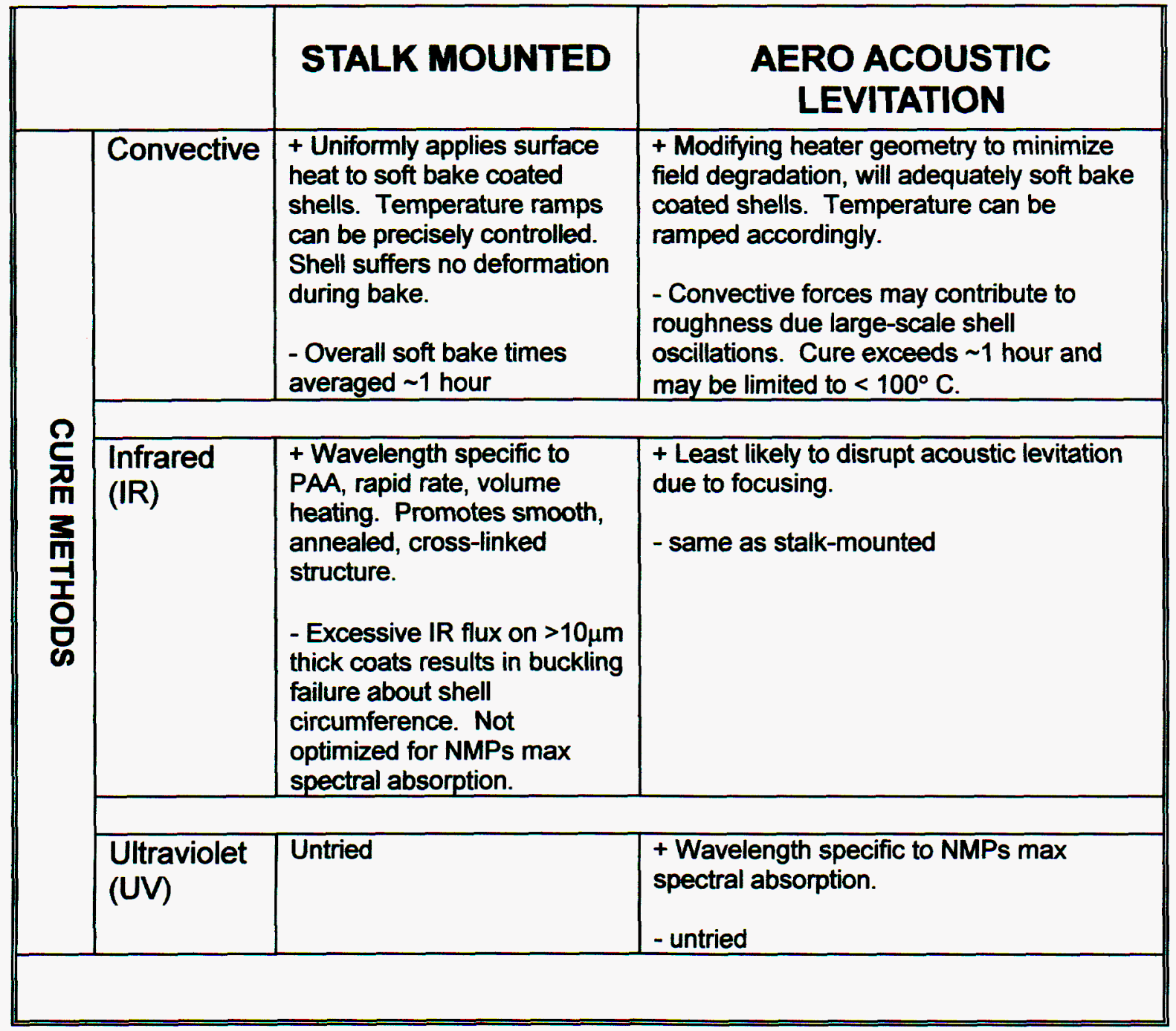

Table 2

515 Tucker Ave.

Friday Harbor, WA 98250 USA
Tel: (360) 378-4137 Fax: (360) 378-4266

wnw.luxel.com

email: luxel@luxel.com 
Over the past 2 years, we have progressed a great deal toward producing solution-based, polyimidecoated NIF quality fuel capsules. We attained a thick $(\sim 84 \mu \mathrm{m})$, fully-imidized P-coated stalkmounted shell using convective curing. While this is a significant achievement, it falls short of our deliverable in terms coating smoothness. Using IR curing methods, we attained coating smoothness approaching our deliverable, yet lacking sufficient thickness. Throughout these efforts, we have gained considerable knowledge and know-how about the details and challenges inherent in solutionbased coating of NIF shells. We likewise developed a considerable facility, which includes characterization techniques and automated coating processes.

We think these achievements provide a strong foundation for continued progress-toward and eventual success-in producing NIF-quality shells. Upon further funding, Luxel will continue pursuing the coating and curing of levitated shells that will meet existing NIF specifications. 